Um esquema upwind polinomial por partes para problemas em

\author{
mecânica dos fluidos
}

Patrícia Sartori 



\title{
Um esquema upwind polinomial por partes para problemas em mecânica dos fluidos
}

\author{
Patrícia Sartori
}

Orientador: Prof. Dr. Valdemir Garcia Ferreira

Dissertação apresentada ao Instituto de Ciências Matemáticas e de Computação - ICMC-USP, como parte dos requisitos para obtenção do título de Mestre em Ciências - Ciências de Computação e Matemática Computacional. VERSÃO REVISADA.

USP - São Carlos

Junho/2011 
Ficha catalográfica elaborada pela Biblioteca Prof. Achille Bassi e Seção Técnica de Informática, ICMC/USP, com os dados fornecidos pelo(a) autor(a)

\begin{tabular}{|c|c|}
\hline \multirow[t]{3}{*}{ s251e } & $\begin{array}{l}\text { Sartori, Patrícia } \\
\text { Um esquema upwind polinomial por partes para } \\
\text { problemas em mecânica dos fluidos / Patrícia Sartori; } \\
\text { orientador Valdemir G. Ferreira -- São Carlos, } 2011 \text {. } \\
\quad 127 \mathrm{p} \text {. }\end{array}$ \\
\hline & $\begin{array}{l}\text { Dissertação (Mestrado - Programa de Pós-Graduação en } \\
\text { Ciências de Computação e Matemática Computacional) -- } \\
\text { Instituto de Ciências Matemáticas e de Computação, } \\
\text { Universidade de São Paulo, 2011. }\end{array}$ \\
\hline & $\begin{array}{l}\text { 1. esquemas upwind de alta resolução. } 2 \text {. esquemas } \\
\text { CBC/TVD. 3. transporte convectivo. 4. escoamentos } \\
\text { incompressiveis. 5. equações de Navier-Stokes. I. G. } \\
\text { Ferreira, Valdemir, orient. II. Título. }\end{array}$ \\
\hline
\end{tabular}


Dedico às pessoas que mais amo na vida, minha família. 



\section{Agradecimentos}

Agradeço primeiramente a Deus pela benção alcançada (o título de mestre).

Aos meus pais, Ricardo e Vanira, e ao meu irmão, Vinícius, que nos momentos mais difíceis acreditaram em mim e, com muito carinho e apoio, foram fundamentais para que eu completasse mais esta etapa da minha vida.

Ao meu namorado e amigo Adriano pela motivação e compreensão nos dias difíceis.

Ao professor Dr. Valdemir Garcia Ferreira pela orientação e incentivo no decorrer do projeto.

A todos os meus amigos e colegas do LCAD - ICMC, em especial a Laís, Josuel, Giseli, Rafael, Larissa e Ítalo pela amizade, companheirismo e grande ajuda. Não poderia deixar de lembrar do meu amigo Miguel, que tenho tanta admiração, pela paciência e auxílio em diversos problemas. Com eles divido o mérito pela conclusão desse trabalho.

A todos os professores do LCAD - ICMC pelos ensinamentos.

Ao apoio financeiro concedido nesses dois anos pelo Conselho Nacional de Desenvolvimento Científico e Tecnológico - CNPq para a realização do meu projeto.

Por fim, a todos que direta ou indiretamente contribuíram para a realização desse trabalho. 


\section{Resumo}

Este trabalho de pesquisa é dedicado ao desenvolvimento, análise e implementação de um novo esquema upwind de alta resolução (denominado PFDPUS) para aproximação de termos convectivos em leis de conservação e problemas relacionados em mecânica dos fluidos. Usando variáveis normalizadas de Leonard, o esquema PFDPUS é baseado em uma função polinomial por partes que satisfaz os critérios de estabilidade CBC e TVD. O desempenho do esquema PFDPUS é investigado na solução das equações de advecção de escalares, Burgers, Euler e MHD. O novo esquema é então aplicado para simular escoamentos incompressíveis envolvendo superfícies livres móveis. Para tanto, o esquema PFDPUS é implementado dentro do software CLAWPACK para problemas compressíveis, e no código Freeflow para problemas incompressíveis. Os resultados numéricos são comparados com dados experimentais, numéricos e analíticos.

Palavras-chave: esquemas upwind de alta resolução; esquemas CBC/TVD; transporte convectivo; escoamentos incompressíveis; equações de Navier-Stokes; simulação numérica. 


\section{Abstract}

This work is dedicated to the development, analysis and implementation of a new highresolution upwind scheme (called PFDPUS) for approximation of convective terms in conservation laws and related fluid mechanics problems. By using the normalized variables of Leonard, the PFDPUS scheme is based on a piecewise polynomial function that satisfies the CBC and TVD stability criteria. The performance of the PFDPUS scheme is assessed by solving advection of scalars, Burgers, Euler and MHD equations. Then the new scheme is applied to simulate incompressible flows involving moving free surfaces. The PFDPUS scheme is implemented into the CLAWPACK software for compressible problems, and in the Freeflow code for incompressible problems. The numerical results are compared with experimental, numerical and analytical data.

Key-works: high resolution upwind scheme; CBC/TVD schemes; convective transport; incompressible flows; Navier-Stokes equations; numerical simulation. 


\section{Sumário}

1 Introdução $\quad 1$

1.1 Estrutura do trabalho . . . . . . . . . . . . . . . . . . . 3

2 Formulação matemática $\quad 5$

2.1 Leis de conservação hiperbólicas $1 \mathrm{D} \ldots \ldots \ldots$. . . . . . . . . . . 5

2.1 .1 Equação de advecção $1 \mathrm{D} \ldots \ldots \ldots$. . . . . . . . . . . . . 6

2.1.2 Equação de Burgers 1D - sem viscosidade . . . . . . . . . . . . 6

2.1.3 Equações de Euler 1D . . . . . . . . . . . . . . . . . . . . 7

2.1.4 Equações MHD 1D . . . . . . . . . . . . . . . . . . . . . 8

2.2 Leis de conservação hiperbólicas $2 \mathrm{D} \ldots \ldots \ldots \ldots$. . . . . . . . . . 8

2.2.1 Equações de águas rasas $2 \mathrm{D} \ldots \ldots \ldots \ldots$. . . . . . . . . . . . . 9

2.2.2 Equações de Euler 2D . . . . . . . . . . . . . . . . . . . . . . 9

2.2 .3 Equações MHD 2D . . . . . . . . . . . . . . . . . . . . . 10

2.3 Equações de Navier-Stokes . . . . . . . . . . . . . . . . . . . . . . . . . 11

2.4 Modelagem da turbulência . . . . . . . . . . . . . . . . . . . . . 12

2.4.1 Equações médias de Reynolds . . . . . . . . . . . . . . . . . . . . . . 12

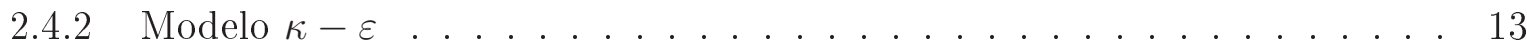

2.5 Estimativas para o erro e ordem de convergência . . . . . . . . . . . . 14

3 Conceitos básicos para o desenvolvimento de esquemas upwind de alta resolução

3.1 Variáveis normalizadas . . . . . . . . . . . . . . . . . 17

3.2 Critério de limitação CBC . . . . . . . . . . . . . . . . . . . . 19

3.3 Restrições TVD . . . . . . . . . . . . . . . . . . . . . . 20

3.4 Formulação dos limitadores de fluxo . . . . . . . . . . . . . . . . . . . . 21

3.5 Esquemas convectivos relevantes . . . . . . . . . . . . . . . . 24

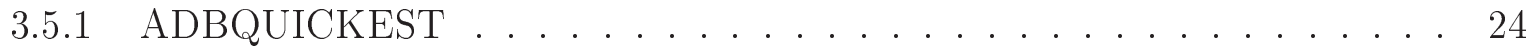




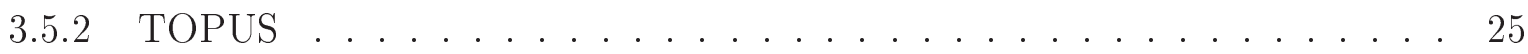

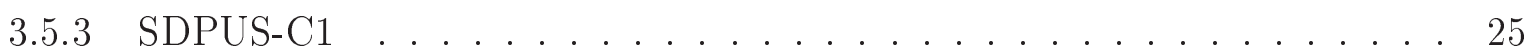

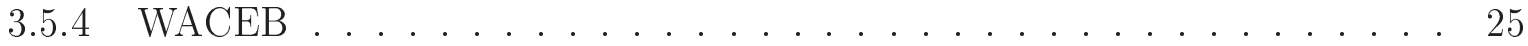

3.5 .5 van Albada . . . . . . . . . . . . . . . . 26

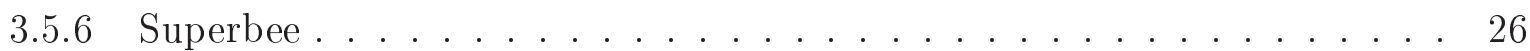

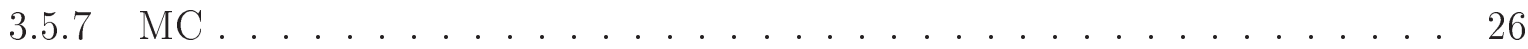

4 O esquema PFDPUS $\quad 27$

4.1 PFDPUS em variáveis normalizadas . . . . . . . . . . . . . . . . . 27

4.2 PFDPUS em variáveis não normalizadas . . . . . . . . . . . . . . . . . . . 29

4.3 Limitador de fluxo do esquema PFDPUS . . . . . . . . . . . . . . . . . . 30

5 Formulação numérica $\quad 35$

5.1 Discretização das leis de conservação 1D . . . . . . . . . . . . . . . . . 35

5.1 .1 Equação de advecção 1D . . . . . . . . . . . . . . . . . 36

5.1 .2 Equação de Burgers 1D - sem viscosidade . . . . . . . . . . . . 36

5.1 .3 Equações de Euler 1D e MHD 1D . . . . . . . . . . . . . . . . 37

5.2 Discretização das leis de conservação $2 \mathrm{D} \ldots \ldots$. . . . . . . . . . . . . 37

5.3 Discretização das equações de Navier-Stokes . . . . . . . . . . . . . . . . 37

5.3 .1 Algoritmo computacional . . . . . . . . . . . . . . . . . 43

6 Resultados numéricos 1D $\quad 47$

6.1 Equação de advecção . . . . . . . . . . . . . . . . . . . . . . . . 47

6.2 Equação de Burgers - sem viscosidade . . . . . . . . . . . . . . . . . 57

6.3 Equações de Euler . . . . . . . . . . . . . . . . . . . . . 65

6.4 Equações MHD . . . . . . . . . . . . . . . . . . . . . . . 69

6.5 Comentários adicionais . . . . . . . . . . . . . . . . 72

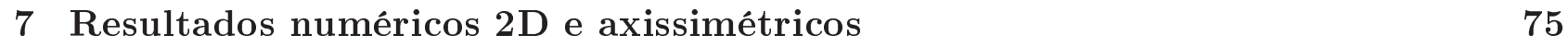

7.1 Leis de conservação 2D . . . . . . . . . . . . . . . . . . . . 75

7.1 .1 Equações de águas rasas . . . . . . . . . . . . . . . . . . 75

7.1 .2 Equações de Euler . . . . . . . . . . . . . . . . . . . . . . . . 79

7.1 .3 Equações MHD . . . . . . . . . . . . . . . . . . . 81

7.2 Escoamentos incompressíveis laminares $2 \mathrm{D} \ldots \ldots \ldots$. . . . . . . . 86

7.2.1 Colapso de uma coluna de fluido . . . . . . . . . . . . . . . . 86

7.2 .2 Jato livre sobre uma superfície rígida impermeável . . . . . . . . . . . 88

7.3 Escoamentos incompressíveis turbulentos 2D . . . . . . . . . . . . . . . 93

7.4 Escoamentos incompressíveis axissimétricos . . . . . . . . . . . . . . . . 96

7.4.1 Experimento de Taylor . . . . . . . . . . . . . . . . . . 96

7.4.2 Ressalto hidráulico circular . . . . . . . . . . . . . . . . . 98 


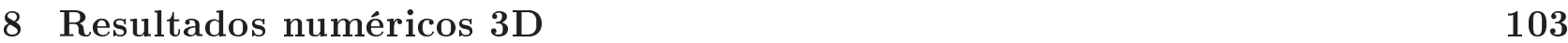

8.1 Jatos oscilantes . . . . . . . . . . . . . . . . . . . . . . . 103

8.2 Ressalto hidráulico circular . . . . . . . . . . . . . . . . . . 108

9 Conclusões e trabalhos futuros $\quad 111$

10 Produção científica 113

10.1 Artigos publicados . . . . . . . . . . . . . . . . . . . 113

10.2 Artigos submetidos . . . . . . . . . . . . . . . . 115

$\begin{array}{ll}\text { A O esquema PFDPUS e os parâmetros livres } \alpha \text { e } \beta & 117\end{array}$

B CLAWPACK 119

$\begin{array}{ll}\text { Referências bibliográficas } & 121\end{array}$ 


\section{Lista de Figuras}

3.1 Posição dos nós computacionais $D, U$ e $R$ conforme o sinal da velocidade $V_{f}$ da

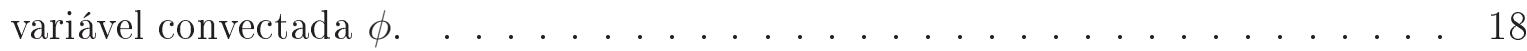

3.2 Diagrama de variáveis normalizadas. . . . . . . . . . . . . . . . . . 19

3.3 Região CBC em variáveis normalizadas. . . . . . . . . . . . . . . . . . . . . . 20

3.4 Região TVD em variáveis normalizadas. . . . . . . . . . . . . . . . . . . . 22

3.5 Representação esquemática dos gradientes consecutivos. . . . . . . . . . . . . 23

3.6 Região TVD - diagrama de Sweby. . . . . . . . . . . . . . . . . . . . 24

4.1 Esquema PFDPUS na região TVD nos planos $\hat{\phi}_{f} \perp \hat{\phi}_{U}$ e $\psi \perp$ r. . . . . . . . 33

5.1 Célula computacional ilustrando o ponto $B$ de discretização dos termos convectivos, bem como, seus vizinhos, as faces $f$ e $g$ para a aproximação e a direção das velocidades $v_{f}$ e $v_{g}$ de convecção. . . . . . . . . . . . . . . . 39

6.1 Comparação dos resultados numéricos obtidos para o problema da condição suave com os métodos de Euler explícito e RK-3 em $t=10$, usando 320 células

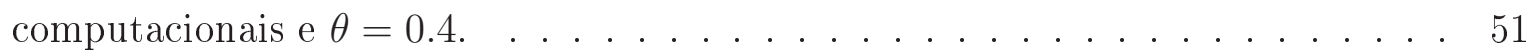

6.2 Resultados numéricos obtidos para o problema de Zalesak em $t=1$ com o esquema PFDPUS, usando 200 células computacionais e $\theta=0.4$. . . . . . . . . 55

6.3 Resultados numéricos obtidos para o problema de Zalesak em $t=1$, usando 200 células computacionais e $\theta=0.4 \ldots \ldots \ldots \ldots$. . . . . . . . . . 56

6.4 Resultados numéricos obtidos para o problema da onda quadrada em $t=2.8$, usando 320 células computacionais e $\theta=0.6 \ldots \ldots \ldots \ldots$. . . . . . 58

6.5 Análise da restrição TVD para o problema da onda quadrada usando o esquema PFDPUS em $t=4 \ldots \ldots \ldots \ldots \ldots \ldots$. . . . . . . . . . . . 59

6.6 Comparação dos resultados numéricos obtidos para o problema da condição suave com os métodos de Euler explícito e RK-3 em $t=1.52$, usando 320 células computacionais e $\theta=0.4$. 
6.7 Resultados numéricos obtidos para o problema do ponto sônico com o esquema PFDPUS em $t=0.3$, usando 200 células computacionais e $\theta=0.7 \ldots \ldots$. . .

6.8 Resultados numéricos obtidos para o problema do ponto sônico em $t=0.3$, usando 200 células computacionais e $\theta=0.7 \ldots \ldots$. . . . . . . . . 63

6.9 Análise da restrição TVD para o problema de Platzman usando o esquema PFDPUS com os parâmetros livres $\alpha$ e $\beta$ em $t=0.25$.

6.10 Resultados numéricos obtidos para o problema do tubo de choque de Sod com o esquema PFDPUS em $t=0.2$, usando 400 células computacionais e $\theta=0.8$. . .

6.11 Resultados numéricos do problema do tubo de choque de Sod para densidade em $t=0.2$, usando 400 células computacionais e $\theta=0.8$.

6.12 Resultados numéricos do problema do tubo de choque de Sod para pressão em $t=0.2$, usando 400 células computacionais e $\theta=0.8 \ldots \ldots \ldots$

6.13 Resultados numéricos do problema das ondas com fortes colisões para a densidade em $t=0.038$, usando 500 células computacionais e $\theta=0.8 \ldots \ldots \ldots$. . .

6.14 Resultados numéricos do problema das ondas com fortes colisões para a pressão em $t=0.038$, usando 500 células computacionais e $\theta=0.8 \ldots \ldots \ldots$. . .

6.15 Resultados numéricos do problema de Riemann de Brio e Wu em $t=0.2$, usando 500 células computacionais e $\theta=0.8 \ldots \ldots \ldots \ldots \ldots$. . . . . . . 72

7.1 Ilustração do problema circular dam-break flow. . . . . . . . . . . . . . . 76

7.2 Resultados numéricos para o problema circular dam-break flow com respeito ao contorno da profundidade $h$ no plano $x \perp y$ e a elevação da superfície de água em $3 \mathrm{D}$ em $t=1.5$ usando uma malha de $125 \times 125$ e $\theta=0.9 \ldots \ldots . . .$.

7.3 Resultados numéricos para o problema circular dam-break flow com respeito ao contorno da profundidade $h$ no plano $x \perp y$ e a elevação da superfície de água em $3 \mathrm{D}$ em $t=1.5$ usando uma malha de $125 \times 125$ e $\theta=0.9 \ldots . . . . .$.

7.4 Comparação dos resultados numéricos, em $t=1.5$, para a variação da profundidade $h$ em função da distância ao longo de $y=0$ para o problema circular dam-break flow. . . . . . . . . . . . . . . . . . .

7.5 Resultados numéricos para o problema das quatro ondas de choque em $t=0.8$ com respeito ao contorno da densidade usando $1000 \times 1000$ células computacionais e $\theta=0.8$.

7.6 Resultados numéricos para o problema das quatro ondas de choque em $t=0.8$ com respeito ao contorno da pressão usando $1000 \times 1000$ células computacionais e $\theta=0.8$.

7.7 Resultados numéricos para o problema two non-neighbouring slip lines em $t=0.3$ com respeito ao contorno da densidade usando $400 \times 400$ células computacionais e $\theta=0.8$. 
7.8 Resultados numéricos para o problema Orszag-Tang em $t=3$ com respeito ao contorno da densidade usando $192 \times 192$ células computacionais e $\theta=0.8$. . . .

7.9 Resultados numéricos para o problema Orszag-Tang em $t=3$ com respeito ao contorno de pressão usando $192 \times 192$ células computacionais e $\theta=0.8$. . . . .

7.10 Comparação entre os resultados de Balbás et al. [3] e os numéricos obtidos para a distribuição da pressão ao longo do corte $y=0.625 \pi$ para o problema de Orszag-Tang em $t=3 \ldots \ldots \ldots \ldots$. . . . . . . . . . 86

7.11 Representação esquemática do problema do colapso de uma coluna de fluido. . . 87

7.12 Comparação entre os dados numéricos, teórico e experimental de Colagrossi e Landrini [11] e o esquema PFDPUS para $x_{\max }$ em função do tempo obtido para o problema do colapso de fluido. . . . . . . . . . . . . . . .

7.13 Resultados numéricos do esquema PFDPUS para a evolução da superfície livre do fluido e campo de pressão nos tempos $t=0.05 \mathrm{~s}, t=0.1 \mathrm{~s}, t=0.15 \mathrm{~s}$ e $t=0.2 \mathrm{~s}$ para o problema do colapso de fluido. . . . . . . . . . . .

7.14 Resultados numéricos do esquema PFDPUS para a evolução da superfície livre do fluido e campo de velocidade na direção $x$ nos tempos $t=0.05 s, t=0.1 s$, $t=0.15 \mathrm{~s}$ e $t=0.2 \mathrm{~s}$ para o problema do colapso de fluido. . . . . . . . .

7.15 Resultados numéricos do esquema PFDPUS para a evolução da superfície livre do fluido e campo de velocidade na direção $y$ nos tempos $t=0.05 s, t=0.1 s$, $t=0.15 \mathrm{~s}$ e $t=0.2 \mathrm{~s}$ para o problema do colapso de fluido. . . . . . . . .

7.16 Representação esquemática do problema do jato livre sobre uma superfície rígida impermeável.

7.17 Comparação entre a solução analítica de Watson [78] e os resultados numéricos do esquema PFDPUS para a altura $H$ entre a superfície rígida e a superfície livre do fluido obtidos para o problema do jato livre.

7.18 Resultados numéricos do esquema PFDPUS para a evolução da superfície livre e campo de pressão nos tempos $t=0.03 \mathrm{~s}, t=0.04 \mathrm{~s}, t=0.05 \mathrm{~s}$ e $t=0.1 \mathrm{~s}$ para o problema do jato livre. . . . . . . . . . . . . . . . .

7.19 Resultados numéricos do esquema PFDPUS para a evolução da superfície livre e campo de velocidade na direção $x$ nos tempos $t=0.03 s, t=0.04 s, t=0.05 \mathrm{~s}$ e $t=0.1 s$ para o problema do jato livre. . . . . . . . . . .

7.20 Resultados numéricos do esquema PFDPUS para a evolução da superfície livre e campo de velocidade na direção y nos tempos $t=0.03 \mathrm{~s}, t=0.04 \mathrm{~s}, t=0.05 \mathrm{~s}$ e $t=0.1 s$ para o problema do jato livre. . . . . . . . . . .

7.21 Comparação entre a solução analítica de Watson [78] e os resultados numéricos do esquema PFDPUS para a altura $H$ entre a superfície rígida e a superfície livre do fluido obtidos para o problema turbulento do jato livre. 
7.22 Resultados numéricos do esquema PFDPUS para a evolução da superfície livre e campo de pressão nos tempos $t=0.04 \mathrm{~s}, t=0.06 \mathrm{~s}, t=0.08 \mathrm{~s}$ e $t=0.15 \mathrm{~s}$ para o problema turbulento do jato livre. . . . . . . . . . . . . . . . . . . . 95

7.23 Resultados numéricos do esquema PFDPUS para a evolução da superfície livre e campo de velocidade na direção $x$ nos tempos $t=0.04 s, t=0.06 s, t=0.08 \mathrm{~s}$ e $t=0.15 \mathrm{~s}$ para o problema turbulento do jato livre. . . . . . . . . . . . 96

7.24 Resultados numéricos do esquema PFDPUS para a evolução da superfície livre e campo de velocidade na direção y nos tempos $t=0.04 s, t=0.06 s, t=0.08 \mathrm{~s}$ e $t=0.15 \mathrm{~s}$ para o problema turbulento do jato livre. . . . . . . . . . . . . 96

7.25 Representação esquemática do experimento de Taylor. . . . . . . . . . . . . . . 97

7.26 Comparação entre os resultados experimentais e numéricos para o experimento de Taylor obtidos com o esquema PFDPUS nos tempos $t=0.75 \mathrm{~s}$ e $t=2.5 \mathrm{~s}$, respectivamente.

7.27 Resultados numéricos do esquema PFDPUS para o experimento de Taylor em $t=10 \mathrm{~s}$

7.28 Resultados numéricos do esquema PFDPUS para a evolução da superfície livre e campos de pressão e velocidade (na direção $r$ e $z$ ) no tempo $t=10 \mathrm{~s}$ para $o$ problema do experimento de Taylor. . . . . . . . . . . . . . . . . . . . . 100

7.29 Representação esquemática do ressalto hidráulico circular. . . . . . . . . . . . . 101

7.30 Comparação entre a solução viscosa de Watson [78] e os resultados numéricos do esquema PFDPUS para a espessura $H$ da camada de fluido.

7.31 Comparação entre o resultados experimental e o numérico obtido com o esquema

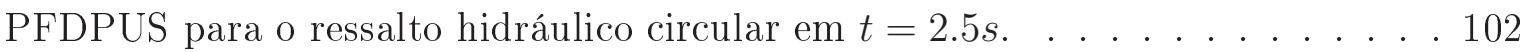

8.1 Representação esquemática dos jatos oscilantes circular e planar. . . . . . . . . . 104

8.2 Resultados numéricos para o jato oscilante circular obtidos com o esquema PFDPUS nos tempos $t=1 s, t=3 s, t=5 s, t=7 s, t=9 s, t=11 s, t=15 s$ e $t=17 s .106$

8.3 Comparação entre os resultados experimentais e numéricos para o jato oscilante circular obtidos com o esquema PFDPUS nos tempos $t=1.5 \mathrm{~s}, t=2.8 \mathrm{~s}, t=4.7 \mathrm{~s}$

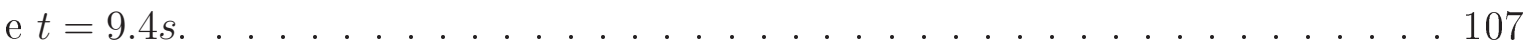

8.4 Resultados numéricos para o jato oscilante planar obtidos com o esquema PFDPUS nos tempos $t=0.3 s, t=0.6 s, t=0.9 s, t=1.2 s, t=1.5 \mathrm{~s}$ e $t=1.8 \mathrm{~s} . \ldots 109$

8.5 Comparação entre o resultados experimental de Ellegard et al. [19] e o numérico obtido com o esquema PFDPUS para o ressalto hidráulico circular em $t=1.8 \mathrm{~s} . \quad 110$ 


\section{Lista de Tabelas}

6.1 Teste de convergência para o problema da condição suave usando-se o esquema PFDPUS com os parâmetros $\alpha$ e $\beta$ em $t=10$. . . . . . . . . . . . . . . 49

6.2 Teste de convergência para o problema da condição suave usando-se os esquemas ABDQUICKEST, TOPUS, SDPUS-C1, WACEB e van Albada em $t=10$. . . . 50

6.3 Teste de convergência para o problema de Zalesak usando-se o esquema PFDPUS com os parâmetros $\alpha$ e $\beta$ em $t=1 \ldots \ldots \ldots \ldots \ldots$. . . . . . . . . . . . . .

6.4 Teste de convergência para o problema de Zalesak usando-se os esquemas ADBQUICKEST, TOPUS, SDPUS-C1, WACEB e van Albada em $t=1$. . . . . . . 54

6.5 Teste de convergência para o problema da condição suave usando-se o esquema

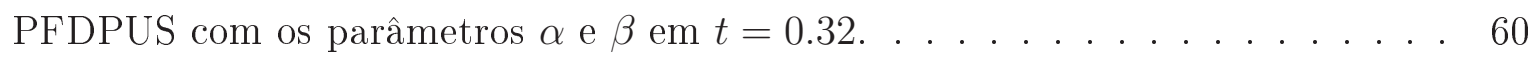

6.6 Teste de convergência para o problema da condição suave usando-se os esquemas ABDQUICKEST, SDPUS-C1 e van Albada em $t=0.32 . \ldots . . . .60$

6.7 Teste de convergência para o problema das ondas com fortes colisões em $t=0.038 .68$

7.1 Teste de convergência para o problema de Orszag-Tang em $t=0.2 \ldots$. . . . . 87 


\section{Introdução}

A mecânica dos fluidos computacional representa uma das áreas de estudo mais importantes em diversos ramos da engenharia. Na maioria das vezes, suas aplicações são caracterizadas por envolver escoamentos com elevados números de Reynolds, especialmente aqueles com superfícies livres móveis. Nos últimos anos, as simulações numéricas vêm se destacando em relação aos métodos analíticos e experimentais para analisar esses problemas. De fato, em muito dos casos, a solução de escoamentos de fluidos com propriedades físicas complexas só pode ser realizada analiticamente quando muitas simplificações são consideradas, o que pode descaracterizar a análise. Por outro lado, testes de laboratório não são suficientes por razões de complexidade, custo e/ou tempo, para possibilitar o entendimento adequado dos fenômenos físicos envolvidos.

Uma das principais dificuldades encontradas no processo de obtenção de soluções numéricas está associada ao tratamento dos termos convectivos presentes nas equações de transporte que descrevem o problema. Consequentemente, a escolha do esquema numérico empregado para discretizar esses termos constitui uma tarefa que pode influenciar diretamente na estabilidade e convergência da solução. Isso requer a utilização de esquemas convectivos que levem em conta a informação física do escoamento (mais conhecidos como esquemas upwind) e que possuam, simultaneamente, características como alta precisão, estabilidade, ausência de oscilações espúrias e simplicidade de implementação.

Em busca dessas características, os estudos nessa área têm se expandido substancialmente e vários esquemas upwind para aproximar termos convectivos estão propostos na literatura. De um lado há os esquemas upwind clássicos de primeira ordem, tais como FOU (First-Order Upwinding) [54] e seu relacionado HDS (Hybrid Differencing Scheme) [63], os quais são altamente estáveis mas possuem características dissipativas indesejáveis (suavização de gradientes). Por outro lado, para remediar os efeitos da dissipação, os esquemas convencionais de alta ordem não limitados, tais como SOU (Second Order Upwind) [76], diferenças centrais e QUICK 
(Quadratic-Upstream Interpolation for Convective Kinematics) [40], são frequentemente utilizados na prática. No entanto, sob condições severas de convecção esses esquemas são vulneráveis a oscilações não físicas e na maioria das vezes ocasionam instabilidades numéricas.

Com o objetivo de superar os problemas numéricos causados pelos esquemas de primeira ordem e os não limitados de alta ordem, atualmente estão sendo desenvolvidos (ver, por exemplo, [32]) esquemas upwind de alta resolução limitados e com pouca introdução de viscosidade artificial. Para tanto, uma estratégia bastante comum é combinar variáveis normalizadas de Leonard [41], ou limitadores de fluxo, com os critérios de limitação CBC (Convection Boundedness Criterion) de Gaskell e Lau [22] e TVD (Total Variation Diminishing) de Harten [26]. A condição TVD assegura variação limitada com o tempo de propriedades físicas e convergência. O critério CBC produz solução limitada.

Como exemplo de esquemas limitados de alta resolução, podem-se citar os esquemas VONOS (Variable-Order Non-Oscillatory Scheme) [75], WACEB (Weighted Average Coefficient Ensuring Boundedness) [62], CUBISTA (Convergent and Universally Bounded Interpolation Scheme for Treatment of Advection) [1], a versão limitada do esquema QUICKEST, chamada ADBQUICKEST (Adaptative Bounded QUICK with Estimated Streaming Terms) [20], e mais recentemente os esquemas TOPUS (Third-Order Polynomial Upwind Scheme) [53] e SDPUS-C1 (Six-Dregree Polinomial Upwind Scheme of $C^{1}$ Class) [15]. Pode-se acrescentar a esta lista o pioneiro MUSCL (Monotone Upstream Scheme for Conservation Laws) [74] e seus relacionados Superbee [57], van Albada [73] e MC (Monotonized Central-difference limiter) [39]. A ideia básica no desenvolvimento desses esquemas é usar um esquema numérico tão preciso quanto possível em regiões suaves e, ao mesmo tempo, introduzir dissipação numérica controlada nas regiões de altos gradientes. Embora a maioria desses esquemas apresentem bons resultados para uma variedade de problemas, alguns deles ainda têm limitações em situações envolvendo descontinuidades (choques) em escoamentos compressíveis (ver [34] e [46]). Outro exemplo aparece em Alves et al. [1], em que os autores ao testar uma variedade de esquemas upwind de alta resolução na simulação de escoamentos viscoelásticos constataram dificuldades de convergência em malha refinadas e forte tendência a oscilar.

É importante observar também que existe a classe dos sofisticados esquemas ENO (Essentially Non-Oscillatory) [30] (e seus relacionados WENO (Weighted Essentially Non-Oscillatory) [4]), que são apropriados para capturar descontinuidades e pontos extremos com alta ordem de precisão. A ideia central desses esquemas consiste na utilização de moléculas de cálculos adaptativas que são escolhidas a partir da suavidade da solução local. No entanto, eles também apresentam algumas desvantagens, uma vez que exigem um elevado número de operações aritméticas para selecionar ou construir uma molécula computacional adequada, o que torna sua implementação cara e difícil.

Nesse contexto, a necessidade de novos esquemas upwind de alta resolução robustos que possuam, simultaneamente, alta precisão, limitação, estabilidade e simplicidade de implemen- 
tação tem sido uma das principais preocupações da comunidade científica moderna em CFD (Computational Fluids Dynamics). Isto constitui a motivação central deste trabalho de mestrado. Outra motivação é dispor de tecnologias competitivas para a simulação de escoamentos incompressíveis com superfícies livres móveis a altos valores do número de Reynolds que ofereçam soluções mais confiáveis, proporcionando melhorias em problemas de interesse prático. Pretende-se também apresentar à literatura especializada dados de simulações para leis de conservação e problemas complexos em dinâmica dos fluidos.

Em particular, este projeto é voltado para a solução numérica de leis de conservação gerais e problemas de escoamentos incompressíveis em regime transitório com superfícies livres móveis. A atenção especial é voltada à derivação de algoritmos upwind de convecção para essa classe de problemas. E os seus principais objetivos são o desenvolvimento, análise e implementação de um novo esquema upwind de alta resolução PFDPUS (Piecewise Fourth Degree Polynomial Upwind Scheme) para a aproximação de termos convectivos (em geral não lineares) de equações de conservação. O novo esquema é uma função polinomial por partes que satisfaz os critérios de estabilidade CBC de Gaskell e Lau [22] e TVD de Harten [26].

Vários testes numéricos são realizados para acessar o desempenho do novo esquema upwind de alta resolução. Dentre eles pode-se citar a simulação de leis de conservação 1D (advecção, Burgers sem viscosidade, Euler e MHD) e 2D (sistemas hiperbólicos de águas rasas, Euler e MHD), e escoamentos incompressíveis com superfícies livres móveis 2D, 3D e axissimétricos. A partir dos resultados numéricos apresentados constata-se que o novo esquema PFDPUS é uma boa alternativa para simular numericamente leis de conservação gerais e problemas em mecânica dos fluidos.

\subsection{Estrutura do trabalho}

O restante do texto está estruturado da seguinte forma:

- O próximo capítulo apresenta as equações básicas utilizadas nesse trabalho;

- No Capítulo 3, descreve-se a base teórica necessária para o desenvolvimento de um esquema upwind de alta resolução, juntamente com os esquemas numéricos que serão utilizados nas simulações;

- O Capítulo 4 é reservado para o desenvolvimento do novo esquema upwind PFDPUS de alta resolução;

- A discretização das equações básicas e o algoritmo computacional para simulação de escoamentos incompressíveis com superfícies livres são apresentados no Capítulo 5;

- O Capítulo 6 engloba os resultados numéricos de leis de conservação 1D;

- Os resultados numéricos para leis de conservação 2D e para escoamentos incompressíveis 2D e axissimétricos com superfícies livres móveis são apresentados no Capítulo 7; 
- No Capítulo 8, são mostrados os resultados para a simulação de escoamentos incompressíveis 3D com superfícies livres móveis laminares;

- As conclusões e as propostas para os trabalhos futuros são descritas no Capítulo 9;

- No Capítulo 10, são listados os artigos publicados e relacionados com a pesquisa apresentada nesse trabalho;

- E, finalmente, nos Apêndices A e B apresentam-se, respectivamente, uma breve descrição de como são determinados os parâmetros livres $\alpha$ e $\beta$ que tornam o esquema PFDPUS TVD e uma síntese do algoritmo de resolução utilizado no software CLAWPACK. 
CAPÍTULO

2

\section{Formulação matemática}

Neste capítulo são descritas as equações básicas essenciais para o desenvolvimento e aplicação dos esquemas numéricos estudados nesse trabalho. Em seguida, apresenta-se uma breve descrição da modelagem $\kappa-\varepsilon$ para simular os efeitos da turbulência. São também apresentados o cálculo dos erros e estimativas para a ordem de convergência.

\subsection{Leis de conservação hiperbólicas 1D}

As leis de conservação são uma classe de equações diferenciais parciais muito importantes que modelam leis de conservação da física como massa, momento, energia, etc. Em geral, elas são não lineares, o que afeta fortemente o procedimento numérico da solução. Segundo Toro [72], no caso 1D, essas leis de conservação são um sistema de equações diferenciais parciais que podem ser escritas da forma

$$
\phi_{t}+F(\phi)_{x}=0
$$

onde

$$
\phi=\left[\begin{array}{c}
\phi_{1} \\
\phi_{2} \\
\vdots \\
\phi_{m}
\end{array}\right] \quad \text { e } \quad F(\phi)=\left[\begin{array}{c}
f_{1} \\
f_{2} \\
\vdots \\
f_{m}
\end{array}\right]
$$

$\phi$ é chamado de vetor das variáveis conservadas, $F=F(\phi)$ é o vetor das funções fluxo e cada uma de suas componentes $f_{i}$ é uma função de componentes $\phi_{j}$ de $\phi$.

Nesta seção, apresentam-se casos particulares das leis de conservação (2.2), tais como, as equações de advecção, Burgers sem viscosidade, Euler e MHD. Para cada uma dessas equações, as condições inciais e de contorno também são descritas. 


\subsubsection{Equação de advecção 1D}

A equação linear de advecção é um dos problemas mais simples decorrentes das leis (2.1) (ver, por exemplo, [50]). Embora seja bastante simples, ela está longe de ser um problema trivial, pois apresenta dificuldades semelhantes àquelas encontradas em sistemas mais complexos. De acordo com (2.1), a equação de advecção é dada tomando-se

$$
\phi=u
$$

$\mathrm{e}$

$$
F(\phi)=a u
$$

onde $a$ é a velocidade de convecção e $u$ é a variável convectada. Nesse trabalho $a$ é constante e igual a 1 em todo o domínio computacional.

As condições iniciais e de contorno consideradas são, respectivamente, da forma

$$
\begin{gathered}
u(x, 0)=u_{0}(x), \\
u\left(x_{L}, t\right)=u_{L} \quad \text { e } \quad u\left(x_{R}, t\right)=u_{R},
\end{gathered}
$$

em que $x \in\left[x_{L}, x_{R}\right]$.

A solução geral para esta equação é

$$
u(x, t)=u_{0}(x-a t)
$$

que consiste simplesmente na translação da condição inicial para direita, sem pertubações, com velocidade de propagação $a>0$ (ou para esquerda se $a<0$ ).

\subsubsection{Equação de Burgers 1D - sem viscosidade}

A equação de Burgers sem viscosidade é uma das mais populares equações não lineares que fornece um simples exemplo das leis (2.1) para capturar o fenômeno crucial da formação do choque. Esse modelo de equação pode apresentar formação do choque mesmo com dados inicias suaves (ver em [29] e [45]). As descontinuidades aparecem na solução após um tempo finito, e, em seguida, se propagam de maneira de irregular fazendo com que a onda, que é inicialmente suave, vá se tornando mais íngreme e acentuada até formar um salto de descontinuidade - o choque.

De acordo com (2.1), a equação de Burgers sem viscosidade é dada escolhendo-se

$$
\phi=u
$$


e

$$
F(\phi)=\frac{1}{2} u^{2}
$$

onde $u$ é a variável dependente semelhante a velocidade em um escoamento incompressível.

As condições iniciais e de contorno adotadas são, respectivamente,

$$
\begin{gathered}
u(x, 0)=u_{0}(x), \\
u\left(x_{L}, t\right)=f\left(x_{L}, t\right) \quad \text { e } \quad u\left(x_{R}, t\right)=g\left(x_{R}, t\right),
\end{gathered}
$$

$\operatorname{com} x \in\left[x_{L}, x_{R}\right]$

\subsubsection{Equações de Euler 1D}

As equações de Euler constituem um sistema de equações hiperbólicas não lineares que governam o escoamento de um fluido compressível e inviscida [21]. Entre outros problemas, descreve o escoamento de um gás em um tubo de choque que tem sido muito investigado em diversos fenômenos físicos, tais como reações químicas e aerodinâmica de veículos supersônicos e hipersônicos.

Neste caso, o sistema de equações é obtido considerando, em (2.1), o vetor de variáveis conservadas e o vetor das funções fluxo dados, respectivamente, como

$$
\phi=[u, \rho u, E]^{T}
$$

e

$$
F(\phi)=\left[\rho u, \rho u^{2}+P, u(E+P)\right]^{T} .
$$

Aqui, $\rho, u, \rho u, P$ e $E$ são, respectivamente, a densidade, a velocidade, o momento, a pressão e energia total. Além disso, para obter um sistema fechado de equações, deve-se considerar a equação de gás ideal

$$
P=(\gamma-1)\left(E-\frac{1}{2} \rho u^{2}\right)
$$

em que $\gamma=1.4$ é a razão do calor específico (Harten [27]).

As condições iniciais adotadas para essas equações são

$$
\left[\rho_{0}, u_{0}, P_{0}\right]^{T}=\left\{\begin{array}{lll}
{\left[\rho_{L}, u_{L}, P_{L}\right]^{T},} & \text { se } & x \leq x_{0} \\
{\left[\rho_{R}, u_{R}, P_{R}\right]^{T},} & \text { se } & x>x_{0}
\end{array}\right.
$$

e as condições de contorno são dadas por reflexão/extrapolação de ordem zero (ver [43]). 


\subsubsection{Equações MHD 1D}

As equações MHD (Magnetohydrodynamic) formam um sistema de equações hiperbólicas não lineares que modelam escoamentos de fluidos na presença de campo magnético. Elas representam o acoplamento das equações da dinâmica com as equações de Maxwell da eletrodinâmica (ver, por exemplo, em [7] e [45]).

Essas equações são obtidas tomando em (2.1) o vetor de variáveis conservadas e das funções fluxo como

$$
\phi=\left[\rho, \rho u, \rho v, \rho w, B_{2}, B_{3}, E\right]^{T}
$$

$\mathrm{e}$

$$
\begin{aligned}
F(\phi)= & {\left[\rho u, \rho u^{2}+P^{*}, \rho u v-B_{1} B_{2}, \rho u w-B_{1} B_{3},\right.} \\
& \left.u B_{2}-v B_{1}, u B_{3}-w B_{1}, u\left(E+P^{*}\right)-B_{1}\left(u B_{1}+v B_{2}+w B_{3}\right)\right]^{T} .
\end{aligned}
$$

Além disso, assume-se $\nabla \cdot \mathbf{B}=0$. Nessas expressões, $\mathbf{v}=[u, v, w]^{T}$ é o vetor velocidade e $\mathbf{B}=\left[B_{1}, B_{2}, B_{3}\right]^{T}$ é vetor campo magnético. A energia total é dada por

$$
E=\frac{1}{2} \rho\|\mathbf{v}\|^{2}+\frac{P}{\gamma-1}+\frac{1}{2}\|\mathbf{B}\|^{2}
$$

e

$$
P^{*}=P+\frac{1}{2}\|\mathbf{B}\|^{2} .
$$

Para esse problema, a razão do calor específico é $\gamma=2$.

As condições iniciais são

$$
\left[\rho_{0}, u_{0}, v_{0}, w_{0}, B_{2}^{0}, B_{3}^{0}, P_{0}\right]^{T}= \begin{cases}{\left[\rho_{L}, u_{L}, v_{L}, w_{L}, B_{2}^{L}, B_{3}^{L}, P_{L}\right]^{T},} & \text { se } \quad x \leq x_{0} \\ {\left[\rho_{R}, u_{R}, v_{R}, w_{R}, B_{2}^{R}, B_{3}^{R}, P_{R}\right]^{T},} & \text { se } \quad x>x_{0}\end{cases}
$$

e as condições de contorno são dadas por extrapolação de ordem zero (ver [43]).

\subsection{Leis de conservação hiperbólicas 2D}

Em duas dimensões, as leis de conservação hiperbólicas têm a forma

$$
\phi_{t}+F(\phi)_{x}+G(\phi)_{y}=0
$$


onde

$$
\phi=\left[\begin{array}{c}
\phi_{1} \\
\phi_{2} \\
\vdots \\
\phi_{m}
\end{array}\right], \quad F(\phi)=\left[\begin{array}{c}
f_{1} \\
f_{2} \\
\vdots \\
f_{m}
\end{array}\right] \quad \text { e } \quad G(\phi)=\left[\begin{array}{c}
g_{1} \\
g_{2} \\
\vdots \\
g_{m}
\end{array}\right] .
$$

$\phi$ é chamado de vetor das variáveis conservadas, e, $F=F(\phi)$ e $G=G(\phi)$ são os vetores das funções fluxo nas direções $x$ e $y$, respectivamente (ver [43]).

Neste trabalho, as leis de conservação 2D consideradas são as equações de águas rasas, Euler e MHD. Essas equações e suas respectivas condições iniciais e de contorno são apresentadas abaixo.

\subsubsection{Equações de águas rasas 2D}

As equações de águas rasas formam um conjunto de equações hiperbólicas não lineares que descrevem escoamentos com superfícies livres, tais como, a ruptura de barragens e os efeitos das ressacas devido a tempestades tropicais e furacões, entre outros (ver [14]).

Em duas dimensões, essas equações podem ser escritas tomando em (2.21) o vetor de variáveis conservadas e das funções fluxo dados, respectivamente, por (ver, por exemplo, [43] e [72])

$$
\begin{gathered}
\phi=[h, h u, h v]^{T}, \\
F(\phi)=\left[h u, h u^{2}+\frac{1}{2} g h, h u v\right]^{T}
\end{gathered}
$$

e

$$
G(\phi)=\left[h v, h u v, h v^{2}+\frac{1}{2} g h^{2}\right]^{T},
$$

em que $h, \mathbf{v}=[u, v]^{T}$ e $g$ são a altura da camada de água, o vetor velocidade e a constante gravitacional, respectivamente.

Neste trabalho, essas equações são suplementadas com condições iniciais da forma

$$
u(x, y, 0)=u_{0}(x, y), \quad v(x, y, 0)=v_{0}(x, y) \quad \mathrm{e} \quad h(x, y, 0)=h_{0}(x, y),
$$

e condições de contorno dadas por extrapolação de ordem zero (ver [43]).

\subsubsection{Equações de Euler 2D}

Em duas dimensões, o sistema de equações de Euler da dinâmica dos gases pode ser escrito de acordo com (2.21), tomando

$$
\begin{gathered}
\phi=[\rho, \rho u, \rho v, E]^{T} \\
F(\phi)=\left[\rho u, \rho u^{2}+P, \rho u v, u(E+P)\right]^{T}
\end{gathered}
$$


e

$$
G(\phi)=\left[\rho v, \rho u v, \rho v^{2}+P, v(E+P)\right]^{T} .
$$

A equação de gás ideal, que é utilizada para fechamento das equações, é

$$
P=(\gamma-1)\left[E-\frac{1}{2} \rho\left(u^{2}+v^{2}\right)\right],
$$

onde $\gamma=1.4$ é a razão do calor específico.

Este problema é resolvido em um domínio quadrado dividido em quatro quadrantes pelas linhas $x=x_{0}$ e $y=y_{0}$. Em cada um desses quadrantes são definidos estados constantes que, resumidamente, são da forma (ver mais detalhes em [36] e [59])

$$
\left[\rho_{0}, u_{0}, v_{0}, P_{0}\right]^{T}=\left\{\begin{array}{llll}
{\left[\rho_{1}, u_{1}, v_{1}, P_{1}\right]^{T},} & \text { se } x>x_{0} & \text { e } y>y_{0}, \\
{\left[\rho_{2}, u_{2}, v_{2}, P_{2}\right]^{T},} & \text { se } x<x_{0} & \text { e } y>y_{0}, \\
{\left[\rho_{3}, u_{3}, v_{3}, P_{3}\right]^{T},} & \text { se } x<x_{0} & \text { e } y<y_{0}, \\
{\left[\rho_{4}, u_{4}, v_{4}, P_{4}\right]^{T},} & \text { se } x>x_{0} & \text { e } y<y_{0}
\end{array}\right.
$$

onde $\left[\rho_{i}, u_{i}, v_{i}, P_{i}\right]^{T}$ é o vetor de propriedades prescrito nos quadrantes $i=1,2,3,4$.

As condições de contorno são dadas por extrapolação de ordem zero (ver [43]).

\subsubsection{Equações MHD 2D}

O sistema de equações MHD, em duas dimensões, admite em (2.21) (ver [3])

$$
\begin{gathered}
\phi=\left[\rho, \rho u, \rho v, \rho w, B_{1}, B_{2}, B_{3}, E\right]^{T}, \\
F(\phi)=\left[\rho u, \rho u^{2}+P^{*}-B_{1}^{2}, \rho u v-B_{1} B_{2}, \rho u w-B_{1} B_{3},\right. \\
\left.0, u B_{2}-v B_{1}, u B_{3}-w B_{1}, u\left(E+P^{*}\right)-B_{1}\left(u B_{1}+v B_{2}+w B_{3}\right)\right]^{T}
\end{gathered}
$$

e

$$
\begin{aligned}
G(\phi)= & {\left[\rho v, \rho v u-B_{2} B_{1}, \rho v^{2}+P^{*}-B_{2}^{2}, \rho v w-B_{2} B_{3}, v B_{1}-u B_{2},\right.} \\
& \left.0, v B_{3}-w B_{2}, v\left(E+P^{*}\right)-B_{2}\left(u B_{1}+v B_{2}+w B_{3}\right)\right]^{T}
\end{aligned}
$$

em que $P^{*}=P+\frac{1}{2}\|\mathbf{B}\|^{2}$. Em adição, a energia total é da forma

$$
E=\frac{1}{2} \rho\|\mathbf{v}\|^{2}+\frac{P}{\gamma-1}+\frac{1}{2}\|\mathbf{B}\|^{2}
$$

onde $\gamma=\frac{5}{3}$.

As condições de contorno são periódicas (ver [43]). 


\subsection{Equações de Navier-Stokes}

As equações de Navier-Stokes são equações diferenciais parciais que modelam escoamentos nos regimes laminar e turbulento. Em coordenadas cartesianas 3D e na notação de Einstein, essas equações são

$$
\begin{aligned}
\frac{\partial u_{i}}{\partial t}+\frac{\partial\left(u_{i} u_{j}\right)}{\partial x_{j}} & =-\frac{\partial P}{\partial x_{i}}+\frac{1}{R_{e}} \frac{\partial}{\partial x_{j}}\left(\frac{\partial u_{i}}{\partial x_{j}}\right)+\frac{1}{F_{r}^{2}} g_{i}, \quad i=1,2,3 \\
\frac{\partial u_{i}}{\partial x_{i}} & =0
\end{aligned}
$$

onde $t$ é o tempo, $u_{i}$ são os componentes do vetor velocidade, $P$ é a pressão e $g_{i}$ são os componentes do campo gravitacional. Os parâmetros adimensionais $R e=\frac{U_{0} L_{0}}{\nu}$ e $F r=\frac{U_{0}}{\left.\sqrt{(} L_{0} / g\right)}$ representam os números de Reynolds e Froude, respectivamente, sendo $\nu=\frac{\mu}{\rho}$ ( $\mu$ é o coeficiente de viscosidade dinâmica e $\rho$ a massa específica) o coeficiente de viscosidade cinemática molecular do fluido. $U_{0}$ e $L_{0}$ são, respectivamente, as escalas de velocidade e de comprimento.

Para os casos com simetria radial (axissimétricos), as equações de Navier-Stokes são dadas em coordenadas cilíndricas da forma

$$
\begin{aligned}
\frac{\partial u}{\partial t}+\frac{1}{r} \frac{\partial(r u u)}{\partial r}+\frac{\partial(u v)}{\partial z} & =-\frac{\partial P}{\partial r}+\frac{1}{R e} \frac{\partial}{\partial z}\left(\frac{\partial u}{\partial z}-\frac{\partial v}{\partial r}\right)+\frac{1}{F r^{2}} g_{r} \\
\frac{\partial v}{\partial t}+\frac{1}{r} \frac{\partial(r v u)}{\partial r}+\frac{\partial(v u)}{\partial z} & =-\frac{\partial P}{\partial z}+\frac{1}{R e} \frac{1}{r} \frac{\partial}{\partial r}\left[r\left(\frac{\partial u}{\partial z}-\frac{\partial v}{\partial r}\right)\right]+\frac{1}{F r^{2}} g_{z} \\
\frac{1}{r} \frac{\partial(r u)}{\partial r}+\frac{\partial v}{\partial z} & =0
\end{aligned}
$$

em que $u=u(r, z, t)$ e $v=v(r, z, t)$ são as componentes do vetor velocidade nas direções $r$ e $z$, respectivamente, e $g=\left[g_{r}, g_{z}\right]^{T}$ representa o campo gravitacional.

As equações de Navier-Stokes são suplementadas com condições iniciais e de contorno (ver detalhes em Tomé e McKee [69]). Nos escoamentos incompressíveis, que são de interesse nesse trabalho, os seguintes tipos de condições de contorno são adotados:

- Na entrada do fluido (injetor): as condições de contorno são

$$
u_{n}=U_{0} \quad \text { e } \quad u_{t}=0
$$

em que $u_{n}$ e $u_{t}$ são as velocidades normal e tangencial ao contorno, respectivamente;

- Na saída do fluido (ejetor): as condições de contorno são

$$
\frac{\partial u_{n}}{\partial n}=0 \quad \text { e } \quad \frac{\partial u_{t}}{\partial n}=0
$$

- No contorno rígido: são considerados dois casos: 
- no-slip: condição de contorno sem escorregamento dadas por

$$
u_{t}=0 \quad \text { e } \quad u_{n}=0
$$

- free-slip: condições de contorno com escorregamento dada por

$$
u_{n}=0 \quad \text { e } \quad \frac{\partial u_{t}}{\partial n}=0
$$

- Na superfície livre: as condições de contorno são

$$
\begin{aligned}
n \cdot(\sigma \cdot n) & =P_{\text {ext }}, \\
m_{1} \cdot(\sigma \cdot n) & =0, \\
m_{2} \cdot(\sigma \cdot n) & =0,
\end{aligned}
$$

em que $P_{\text {ext }}$ é a pressão externa (atmosférica local), que é assumida zero neste trabalho. Para o caso $3 \mathrm{D}, m_{1}=\left(m_{1 x}, m_{1 y}, m_{1 z}\right)$ e $m_{2}=\left(m_{2 x}, m_{2 y}, m_{2 z}\right)$ são os vetores tangentes à superfície livre, e, $n=\left(n_{x}, n_{y}, n_{z}\right)$ é o vetor unitário normal exterior à superfície livre. Para o caso 2D, $m_{1}=\left(m_{1 x}, m_{1 y}\right)$ e $n=\left(n_{x}, n_{y}\right)$, e para o caso axissimétrico, $m_{1}=\left(-n_{z}, 0, n_{r}\right)$ e $n=\left(n_{r}, 0, n_{z}\right)$. Aqui $\sigma$ é o tensor de tensões total, que em 2D e 3D é da forma

$$
\sigma=-P I+2 \mu\left(\nabla u+(\nabla u)^{T}\right)
$$

sendo $I$ o tensor identidade. Para o caso axissimétrico o tensor é

$$
\sigma=-P I+\frac{1}{R e}\left(\nabla u+(\nabla u)^{T}\right)
$$

\subsection{Modelagem da turbulência}

Neste trabalho, para a modelagem da turbulência são consideradas as equações médias de Reynolds e adota-se a modelagem $\kappa-\varepsilon$, a qual é baseada no conceito de viscosidade turbulenta de Boussinesq [5]. Uma breve introdução à esses conceitos é apresentada nesta seção.

\subsubsection{Equações médias de Reynolds}

Para a simulação dos efeitos da turbulência, as leis físicas de conservação (2.36) e (2.37), no caso 2D, são transformadas nas equações médias de Reynolds

$$
\begin{aligned}
\frac{\partial \bar{u}_{i}}{\partial t}+\frac{\partial\left(\bar{u}_{i} \bar{u}_{j}\right)}{\partial x_{j}} & =-\frac{\partial \bar{P}}{\partial x_{i}}+\frac{1}{R_{e}} \frac{\partial}{\partial x_{j}}\left(\frac{\partial \bar{u}_{i}}{\partial x_{j}}\right)+\frac{1}{F_{r}^{2}} g_{i}-\frac{1}{R e} \frac{\overline{\partial u_{i}^{\prime} u_{j}^{\prime}}}{\partial x_{j}}, \quad i=1,2, \\
\frac{\partial \bar{u}_{i}}{\partial x_{i}} & =0
\end{aligned}
$$


onde $\bar{u}_{i}$ é a i-ésima componente da velocidade média e $\bar{P}$ é a pressão média. O tensor de tensões $\overline{u_{i}^{\prime} u_{j}^{\prime}}$ pode ser expresso pela aproximação de Boussinesq [5] como

$$
\overline{u_{i}^{\prime} u_{j}^{\prime}}=-\nu_{t} D_{i j}+\frac{2}{3} \kappa \delta_{i j}, \quad i=1,2,
$$

em que

$$
\nu_{t}=C_{\mu} \frac{\kappa^{2}}{\varepsilon} \quad \text { e } \quad D_{i j}=\frac{\partial \bar{u}_{i}}{\partial x_{j}}+\frac{\partial \bar{u}_{j}}{\partial x_{i}},
$$

sendo $\delta_{i j}$ o operador delta de Kronecher, $\nu_{t}$ a viscosidade turbulenta local e $C_{\mu}$ uma constante empírica. A energia cinética da turbulência $\kappa$ e a dissipação de energia cinética $(\kappa) \varepsilon$ são definidos, respectivamente, da forma

$$
\kappa=\frac{1}{2} \overline{u_{i}^{\prime} u_{i}^{\prime}} \quad \text { e } \quad \varepsilon=\nu\left(\overline{\frac{\partial u_{i}}{\partial x_{j}}}\right)^{2}
$$

em que $u_{i}^{\prime}$ é a componente flutuante da velocidade $u_{i}$.

É importante observar que as equações (2.50) e (2.51) constituem um sistema de equações diferencias parciais incompleto e, portanto, necessitam de mais uma hipótese para o fechamento. Assim, para o fechamento das equação médias de Reynolds é preciso encontrar uma equação para $\nu_{t}$ que depende da posição e do tempo. Para tanto, é utilizado o chamado modelo $\kappa-\varepsilon$ que permite o conhecimento das variáveis $\kappa$ e $\varepsilon$ para a avaliação da viscosidade turbulenta $\nu_{t}$ (ver detalhes em Brandi [6] e Kurokawa [38]).

\subsubsection{Modelo $\kappa-\varepsilon$}

A ideia principal desse modelo é acoplar às equações médias de Reynolds duas equações diferenciais de transporte para descrever a viscosidade turbulenta $\nu_{t}$. Dessa forma, a determinação das variáveis $\kappa$ e $\varepsilon$ é feita pela resolução das equações

$$
\begin{aligned}
& \frac{\partial \kappa}{\partial t}+\frac{\partial\left(\kappa u_{j}\right)}{\partial x_{j}}=\frac{1}{R e} \frac{\partial}{\partial x_{j}}\left[\left(1+\frac{\nu_{t}}{\sigma_{k}}\right) \frac{\partial \kappa}{\partial x_{j}}\right]+\nu_{t} D_{i j} \frac{\partial u_{i}}{\partial x_{j}}-\varepsilon \\
& \frac{\partial \varepsilon}{\partial t}+\frac{\partial\left(\varepsilon u_{j}\right)}{\partial x_{j}}=\frac{1}{R e} \frac{\partial}{\partial x_{j}}\left[\left(1+\frac{\nu_{t}}{\sigma_{\varepsilon}}\right) \frac{\partial \varepsilon}{\partial x_{j}}\right]+\frac{\varepsilon}{\kappa}\left(C_{1 \varepsilon} \nu_{t} D_{i j} \frac{\partial u_{i}}{\partial x_{j}}-C_{2 \varepsilon} \varepsilon\right)
\end{aligned}
$$

em que $\sigma_{\kappa}=1.0$ e $\sigma_{\varepsilon}=1.3$ são os coeficientes de difusão turbulentos, $C_{1 \varepsilon}=1.44, C_{2 \varepsilon}=1.92 \mathrm{e}$ $C_{\mu}=0.09$ são as constantes empíricas.

Neste trabalho, as condições iniciais e de contorno adotadas para as variáveis médias de velocidade e pressão são as mesmas que as apresentadas na Seção 2.3. Já para as variáveis turbulentas, as condições iniciais e de contorno adotadas são apresentadas a seguir. 
As condições iniciais são (ver Brandi [6])

$$
\kappa=0.08 R e \quad \text { e } \quad \varepsilon=\frac{100 \kappa}{3} \sqrt{\frac{\kappa}{R e}},
$$

e as condições de contorno são dadas por:

- Na entrada do fluido (injetor): as condições de contorno para $\kappa$ e $\varepsilon$ coincidem com (2.57);

- Na saída do fluido (ejetor): para o cálculo das variáveis turbulentas são aplicadas as condições de Neumann homogênea

$$
\frac{\partial \kappa}{\partial n}=0 \quad \text { e } \quad \frac{\partial \varepsilon}{\partial n}=0
$$

- Na superfície rígida: próximo ao contorno rígido, $\kappa$ e $\varepsilon$ são calculados aplicando-se uma modificação das leis clássicas de parede (ver [18], [61], e [6]);

- Na superfície livre do fluido: as condições de contorno são

$$
\begin{array}{r}
P+\frac{2}{3 R e} \kappa-\frac{2}{R e}\left(1+\nu_{t}\right)\left[\frac{\partial u}{\partial x} n_{x}^{2}+\frac{\partial v}{\partial y} n_{y}^{2}+\left(\frac{\partial u}{\partial y}+\frac{\partial v}{\partial x}\right) n_{x} n_{y}\right]=0 \\
\frac{1}{R e}\left(1+\nu_{t}\right)\left[2 \frac{\partial u}{\partial x} m_{x} n_{x}+2 \frac{\partial v}{\partial y} m_{y} n_{y}+\left(\frac{\partial u}{\partial y}+\frac{\partial v}{\partial x}\right)\left(m_{x} n_{y}+m_{y} n_{x}\right)\right]=0 .
\end{array}
$$

\subsection{Estimativas para o erro e ordem de convergência}

Com o objetivo de verificar a precisão dos esquemas utilizados nesse trabalho, apresentam-se aqui as maneiras de calcular os erros e ordens de convergência.

Considerando que a solução exata (ou numérica de referência) é conhecida, os erros absolutos em uma malha de espaçamento $h$, nas normas $L_{1}$ e $L_{2}$, são respectivamente definidos por

$$
\begin{gathered}
\left\|E_{h}\right\|_{1}=\|u-\hat{u}\|_{1}=h \sum_{i=1}^{N}\left|u_{i}-\hat{u}_{i}\right|, \\
\left\|E_{h}\right\|_{2}=\|u-\hat{u}\|_{2}=\sqrt{h \sum_{i=1}^{N}\left(u_{i}-\hat{u}_{i}\right)^{2}},
\end{gathered}
$$

onde $u$ é a solução numérica e $\hat{u}$ é a solução exata avaliada nos mesmos pontos de malha. No caso particular das equações de Euler 1D, os erros nas normas $L_{1}$ e $L_{2}$ são obtidos na forma relativa, respectivamente por

$$
\left\|E_{h}\right\|_{1}=\frac{\|u-\hat{u}\|_{1}}{\|\hat{u}\|_{1}}=h \frac{\sum_{i=1}^{N}\left|u_{i}-\hat{u}_{i}\right|}{\sum_{i=1}^{N}\left|\hat{u}_{i}\right|}
$$




$$
\left\|E_{h}\right\|_{2}=\frac{\|u-\hat{u}\|_{2}}{\|\hat{u}\|_{2}}=\sqrt{\frac{\sum_{i=1}^{N}\left(u_{i}-\hat{u}_{i}\right)^{2}}{\sum_{i=1}^{N}\left(\hat{u}_{i}\right)^{2}}} .
$$

Por simplicidade, a partir de agora os subíndices referentes as normas utilizadas para o cálculo dos erros são omitidos.

Assumindo que o erro se comporta assintoticamente e que o método tem ordem $p$ de precisão então, espera-se, que

$$
\left\|E_{h}\right\|=C h^{p}+O\left(h^{p}\right), \quad \text { quando } \quad h \longrightarrow 0
$$

e se $h$ é suficientemente pequeno então (ver Thomas [68])

$$
\left\|E_{h}\right\| \approx C h^{p}
$$

Ao refinar a malha usando um fator 2, obtém-se

$$
\left\|E_{\frac{h}{2}}\right\| \approx C\left(\frac{h}{2}\right)^{p}
$$

e então, dividindo-se (2.66) por (2.67), segue

$$
\frac{\left\|E_{h}\right\|}{\left\|E_{\frac{h}{2}}\right\|} \approx 2^{p}
$$

Agora, aplicando log em ambos os membros de (2.68), obtém-se

$$
p \approx \frac{\log \left(\frac{\left\|E_{h}\right\|}{\left\|E_{\frac{h}{2}}\right\|}\right)}{\log 2} .
$$

No caso em que a solução exata não é conhecida, uma estimativa para ordem de convergência pode ser calculada com base em três soluções numéricas obtidas em malhas com espaçamentos $h, h / 2$ e $h / 4$ por

$$
p \approx \frac{\log \left(\frac{\left\|E_{h}\right\|}{\left\|E_{\frac{h}{2}}\right\|}\right)}{\log 2}=\frac{\log \left(\frac{\left\|u^{h}-u^{h / 2}\right\|}{\left\|u^{h / 2}-u^{h / 4}\right\|}\right)}{\log 2},
$$

em que $u^{h}, u^{h / 2}$ e $u^{h / 4}$ são, respectivamente, a solução numérica em uma malha mais grossa, a solução numérica em uma malha intermediária e a solução numérica em uma malha mais fina. 


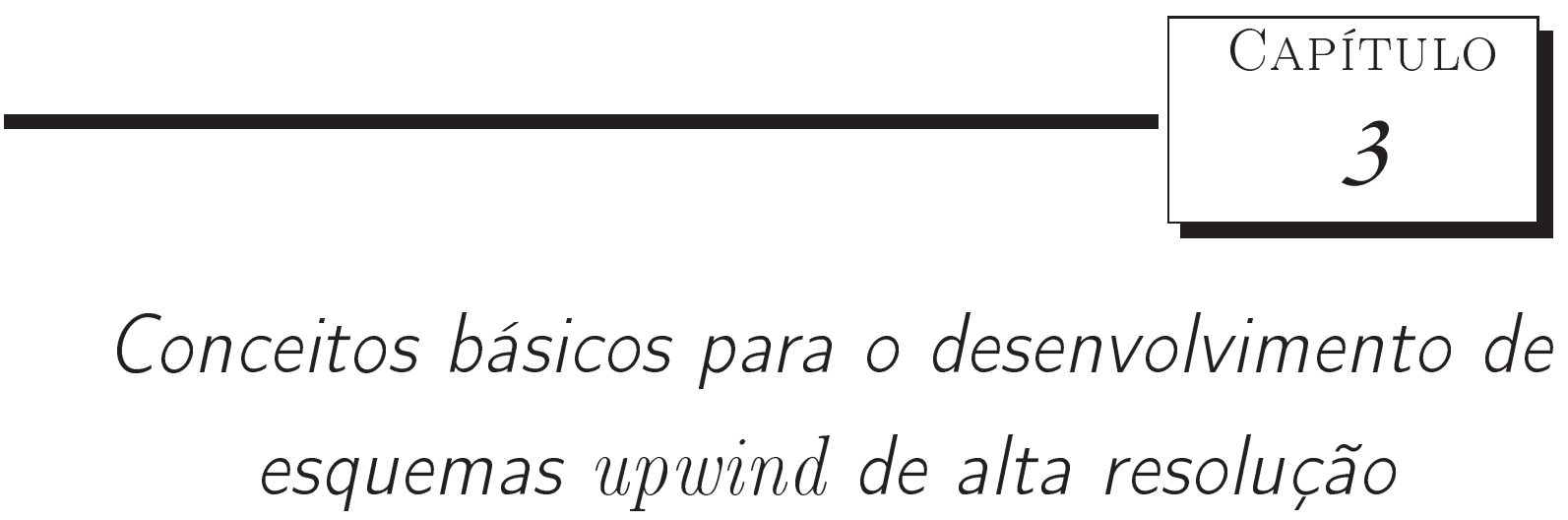

Este capítulo trata dos princípios fundamentais para o desenvolvimento de esquemas upwind de alta resolução. Inicialmente, apresentam-se o conceito de variáveis normalizadas de Leonard [41], o critério CBC de Gaskell e Lau [22] e as restrições TVD de Harten [26]. Em seguida, descrevem-se os principais conceitos para a derivação dos limitadores de fluxo e os esquemas numéricos de alta resolução utilizados neste trabalho.

\subsection{Variáveis normalizadas}

As variáveis normalizadas foram introduzidas por Leonard [41] e têm como objetivo reduzir o número explícito de variáveis na representação de uma propriedade física genérica $\phi$ em uma interface $f$ da célula computacional.

Neste trabalho, a propriedade $\phi$ é interpolada usando valores vizinhos dessa propriedade e informação física do problema, isto é, de maneira upwind. Para tanto, considera-se uma molécula computacional de três pontos: $D$ (Downstream), $U$ (Upstream) e $R$ (Remote-upstream), que são, respectivamente, as posições a jusante, a montante e mais a montante da interface $f$ onde se deseja estimar a propriedade $\phi$. Esses pontos, ilustrados na Figura 3.1, são pré-definidos de acordo com o sinal da velocidade (de convecção) $V_{f}$ da variável convectada $\phi$ em $f, \phi_{f}$.

Dessa forma, a aproximação upwind (esquema convectivo) que faz uso dos pontos $D, U$ e $R$ para avaliar $\phi_{f}$ pode ser representada na forma não normalizada por

$$
\phi_{f}=\phi_{f}\left(\phi_{D}, \phi_{U}, \phi_{R}\right)
$$



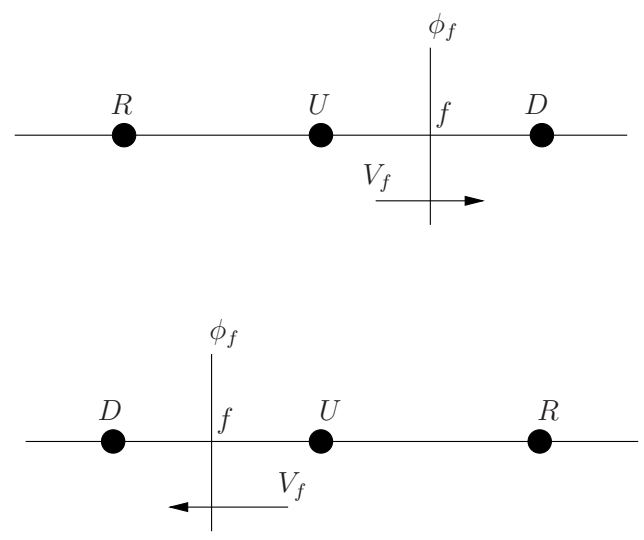

Figura 3.1: Posição dos nós computacionais $D, U$ e $R$ conforme o sinal da velocidade $V_{f}$ da variável convectada $\phi$.

e na forma normalizada, devido à Leonard [41], como

$$
\hat{\phi}_{f}=\hat{\phi}_{f}\left(\hat{\phi}_{U}\right)
$$

onde a variável normalizada $\hat{\phi}_{()}$é dada da forma

$$
\hat{\phi}_{()}=\frac{\phi_{()}-\phi_{R}}{\phi_{D}-\phi_{R}}
$$

Note que em (3.2) $\hat{\phi}_{f}$ depende somente de $\hat{\phi}_{U}$ (informação upwind), desde que $\hat{\phi}_{R}=0$ e $\hat{\phi}_{D}=1$.

Para representar a relação funcional entre $\hat{\phi}_{f}$ e $\hat{\phi}_{U}$, a partir da definição (3.3) e da relação (3.2), Leonard [41] construiu o chamado diagrama de variáveis normalizadas (NVD - Normalized Variable Diagram), que é um conceito útil para a derivação de esquemas de convecção. Para exemplificar, na Figura 3.2 os esquemas FOU [54], diferença central de segunda ordem e QUICK [40] estão representados nesse diagrama.

Nesse contexto, Leonard [41] afirma que os esquemas em variáveis normalizadas que têm intersecção com o segundo quadrante podem produzir oscilações numéricas não físicas e os que interceptam o quarto quadrante podem apresentar difusividade artificial. Assim, para que um esquema seja livre desses problemas, faz-se necessário definir a relação funcional (3.2) no ponto $O=(0,0)$. Experimentos numéricos mostram também que os esquemas em NVD que interceptam a reta $\hat{\phi}_{U}=1$ acima de $\hat{\phi}_{f}=1$ geram oscilações e aqueles que interceptam a reta $\hat{\phi}_{U}=1$ abaixo de $\hat{\phi}_{f}=1$ adicionam viscosidade numérica. Então, uma condição necessária para que um esquema em NVD não apresente esse tipo de problema é que este esteja definido no ponto $P=(1,1)$. Leonard [41] (ver também Waterson e Deconink [77] e Lin e Chieng [47]) demostrou também, por meio de seus estudos, que uma condição necessária e suficiente para que um esquema apresente segunda ordem de precisão é que a relação funcional (3.2) esteja definida no ponto $Q=\left(\frac{1}{2}, \frac{3}{4}\right)$; e mais, se a inclinação no ponto $Q$ é $\frac{3}{4}$, então o esquema é de 


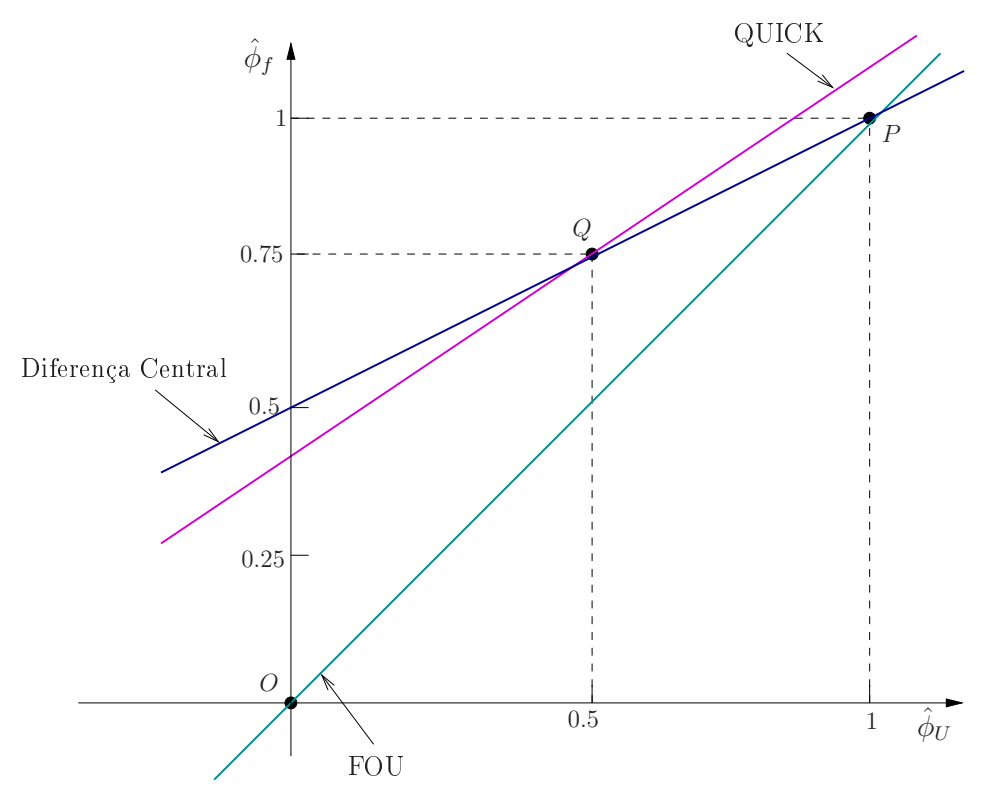

Figura 3.2: Diagrama de variáveis normalizadas.

terceira ordem.

Resumindo: para se conseguir um esquema upwind no contexto NVD (não linear) que seja monotônico e que alcance terceira ordem, a relação funcional (3.2) deve satisfazer as seguintes condições:

- estar definida em $O=(0,0)$ (condição necessária);

- estar definida em $P=(1,1)$ (condição necessária);

- estar definida em $Q=\left(\frac{1}{2}, \frac{3}{4}\right)$ (condição necessária e suficiente para segunda ordem);

- estar definida em $Q$ com inclinação $\frac{3}{4}$ (condição necessária e suficiente para terceira ordem).

Leonard ainda recomenda que, para $\hat{\phi}_{U} \notin[0,1]$, os esquemas devem ser estendidos de maneira contínua pelo esquema FOU [54]. Além disso, para se evitar problemas de convergência em malhas grosseiras, Lin e Cheing [47] recomendam que a relação funcional (3.2) seja continuamente diferenciável em todo domínio computacional.

\subsection{Critério de limitação CBC}

O critério de limitação CBC (Convection Boundedness Criterion) foi proposto por Gaskell e Lau [22] e sua importância é fornecer uma condição necessária e suficiente para que as soluções numéricas obtidas com o uso de um esquema convectivo sejam limitadas. Uma solução é limitada se o seu valor em um ponto computacional não ultrapassa os valores da solução nos pontos vizinhos inerentes ao processo físico.

Dessa forma, um esquema definido por uma função contínua ou contínua por partes $\hat{\phi}_{f}=$ 
$\hat{\phi}_{f}\left(\hat{\phi}_{U}\right)$ é limitado se as seguintes condições são cumpridas:

$$
\left\{\begin{array}{lll}
\hat{\phi}_{f} \in\left[\hat{\phi}_{U}, 1\right], & \text { se } & \hat{\phi}_{U} \in[0,1], \\
\hat{\phi}_{f}=0, & \text { se } & \hat{\phi}_{U}=0, \\
\hat{\phi}_{f}=1, & \text { se } & \hat{\phi}_{U}=1, \\
\hat{\phi}_{f}=\hat{\phi}_{U}, & \text { se } & \hat{\phi}_{U} \notin[0,1] .
\end{array}\right.
$$

A Figura 3.3 ilustra geometricamente a região CBC definida por (3.4). De acordo com Gaskell e Lau [22], o gráfico que define o esquema limitado $\hat{\phi}_{f}\left(\hat{\phi}_{U}\right)$ deve estar inteiramente contido nessa região (a recíproca é verdadeira).

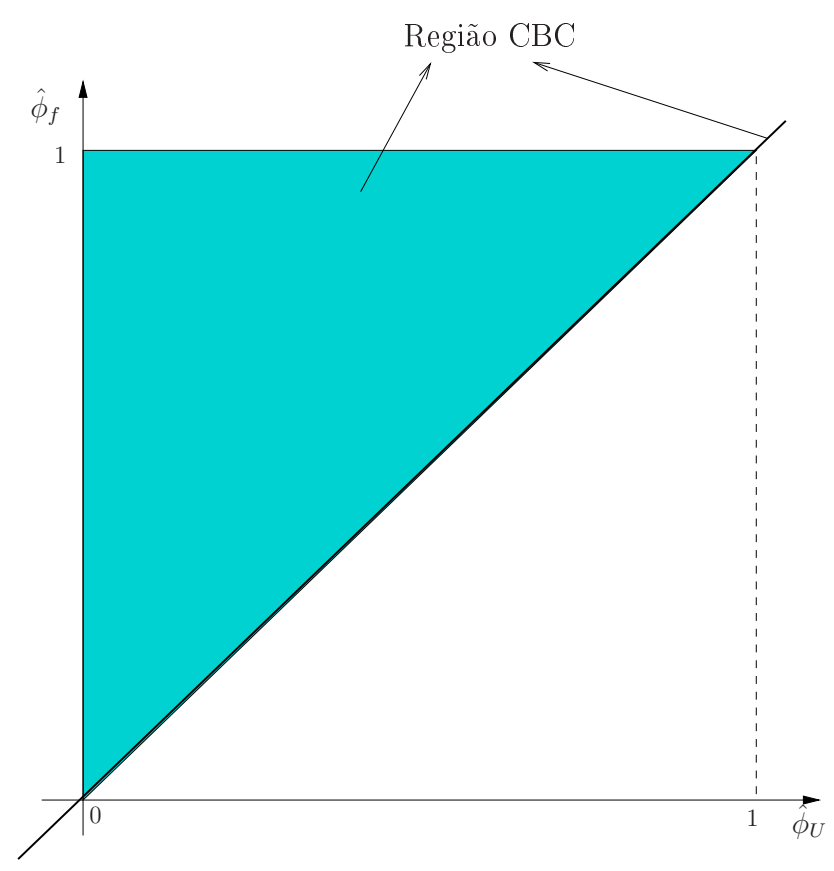

Figura 3.3: Região CBC em variáveis normalizadas.

É importante ressaltar que o critério CBC, apesar de garantir soluções limitadas, não oferece nenhuma garantia de convergência. A convergência é garantida pelas restrições TVD que estão descritas na próxima seção.

\subsection{Restrições TVD}

Ao se falar dos conceitos fundamentais para o desenvolvimento de esquemas upwind de alta resolução é essencial discutir o princípio TVD (Total Variation Diminisching) introduzido por Harten [26]. Métodos que possuem essa característica combinam monotonicidade e alta ordem de precisão.

Considerando uma sequência de aproximações discretas

$$
\phi(t)=\phi_{i}(t)_{i \in \mathbb{Z}}
$$


a Variação Total (TV - Total Variation) de uma solução discreta no tempo $t$ é dada por

$$
T V(\phi(t))=\sum_{i \in \mathbb{Z}}\left|\phi_{i+1}(t)-\phi_{i}(t)\right|
$$

Seja o esquema de diferenças explícito com $(2 k+1)$ pontos da forma

$$
\phi_{i}^{n+1}=H\left(\phi_{i-k}^{n}, \ldots, \phi_{i+k}^{n}\right), \quad \forall n \geq 0, i \in \mathbb{Z}
$$

onde $H: \mathbb{R}^{2 k+1} \longrightarrow \mathbb{R}$ é uma função contínua e $\phi_{i}^{n}$ é a aproximação da solução exata $\Phi$ nos pontos de malha (uniforme) $\left(x_{i}, t_{n}\right)$, com $x_{i}=i \delta x$ e $t_{n}=n \delta t$ ( $\delta x$ o passo espacial e $\delta t$ a marcha no tempo). O esquema (3.7) é dito TVD se

$$
T V\left(\phi^{n+1}\right) \leq T V\left(\phi^{n}\right)
$$

De acordo com Harten, um esquema TVD apresenta as seguintes propriedades: um novo extremo local (máximo ou mínimo) não pode ser criado; o valor do mínimo local não pode ser diminuído; e o valor do máximo local não pode ser aumentado. Esses são princípios básicos de esquemas que requerem preservação de monotonicidade. Em resumo, para esquemas TVD a variação total da solução não deve aumentar. Nesse contexto, LeVeque [43] afirma que se o método é TVD, então em particular dados que são inicialmente monotônicos permanecerão com essa característica nos passos temporais futuros. Dessa maneira, se uma descontinuidade for detectada, a solução numérica não apresentará oscilações nas suas proximidades.

Os conceitos introduzidos por Harten [26] foram reinterpretados por Sweby [64] no contexto NVD. Isso deu origem ao conjunto de restrições TVD

$$
\left\{\begin{array}{lll}
\hat{\phi}_{f} \in\left[\hat{\phi}_{U}, 2 \hat{\phi}_{U}\right] \quad \text { e } \quad \hat{\phi}_{f} \leq 1, & \text { se } & \hat{\phi}_{U} \in[0,1] \\
\hat{\phi}_{f}=\hat{\phi}_{U}, & \text { se } & \hat{\phi}_{U} \notin[0,1]
\end{array}\right.
$$

as quais definem a região mostrada na Figura 3.4. Em suma, para que um esquema definido pela relação funcional $\hat{\phi}_{f}=\hat{\phi}_{f}\left(\hat{\phi}_{U}\right)$ seja TVD ele deve estar inteiramente contido nessa região (a recíproca é verdadeira).

\subsection{Formulação dos limitadores de fluxo}

Concluída a derivação do esquema em NVD, a correspondente formulação do limitador de fluxo pode ser obtida reescrevendo o esquema em variáveis normalizadas (ver Sweby [64] e Waterson e Deconinck [77]) da seguinte forma

$$
\hat{\phi}_{f}=\hat{\phi}_{U}+\frac{1}{2} \psi(r)\left(1-\hat{\phi}_{U}\right)
$$




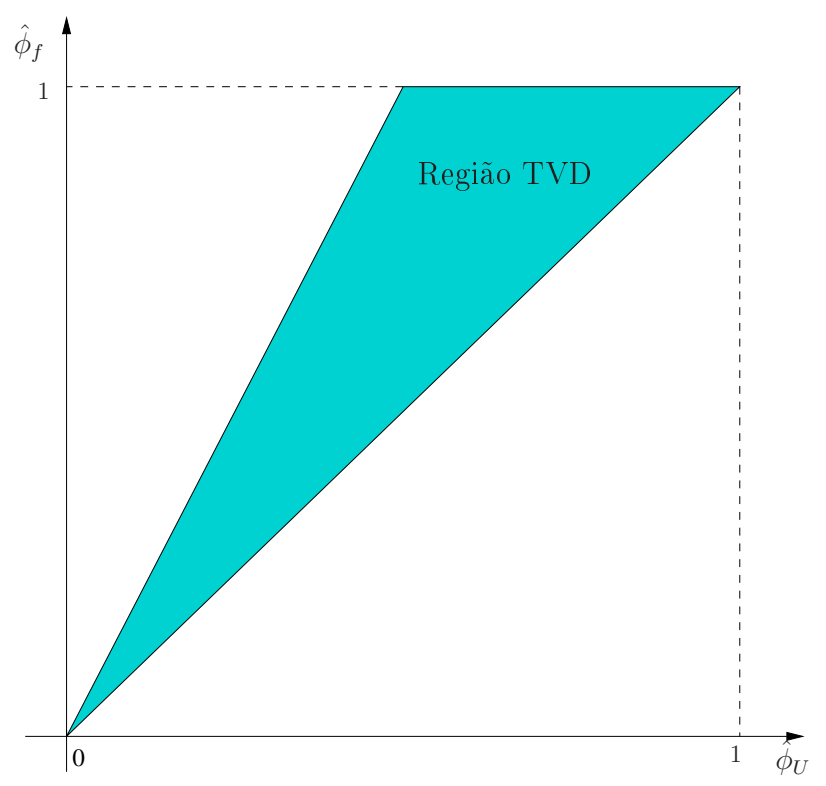

Figura 3.4: Região TVD em variáveis normalizadas.

onde $\psi(r)$ é chamado limitador de fluxo e $r$ é a razão dos gradientes consecutivos (uma espécie de sensor que detecta a variação dos gradientes) dado por (ver Figura 3.5)

$$
r=\frac{\left(\frac{\partial \phi}{\partial x}\right)_{g}}{\left(\frac{\partial \phi}{\partial x}\right)_{f}}
$$

A equação (3.11) em malhas uniformes pode ser aproximada

$$
r=\frac{\phi_{U}-\phi_{R}}{\phi_{D}-\phi_{U}}
$$

e sua normalização torna-se

$$
r=\frac{\hat{\phi}_{U}}{1-\hat{\phi}_{U}}
$$

A ideia básica dessa abordagem é combinar métodos de primeira ordem e de ordem superior com um limitador de fluxo $\psi(r)$ que age como uma função de troca e tem a finalidade de corrigir o fluxo quando houver necessidade. Ou seja, essa estratégia tem como principal objetivo recuperar soluções suaves e, ao mesmo tempo, melhorar a ordem de convergência.

Algumas propriedades podem ser impostas para a construção dos limitadores. Uma delas, de acordo com Waterson e Deconinck [77], é que um esquema convectivo deve respeitar exatamente a variação linear da solução, isto é

$$
\psi(1)=1
$$




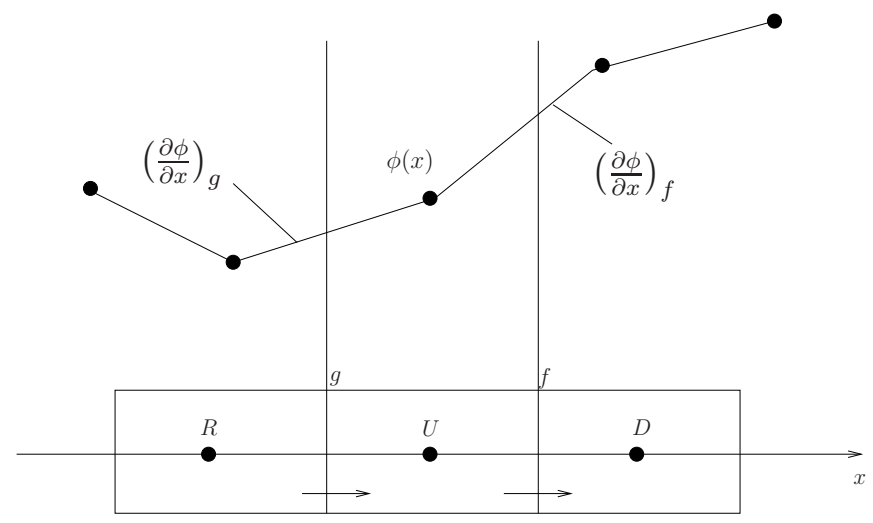

Figura 3.5: Representação esquemática dos gradientes consecutivos.

Em uma malha uniforme essa condição implica em segunda ordem de precisão.

Zijlema [81] propôs um critério sobre o limitador que é condição necessária e suficiente para que o esquema convectivo alcance terceira ordem de precisão. A condição é

$$
\psi^{\prime}(1)=\frac{1}{4}
$$

Zilema [81] ainda afirma que se o limitador $\psi(r)$ é uma função suave de $r$, então o esquema tem melhor comportamento com respeito a convergência no decorrer do processo numérico.

Além disso, as restrições TVD também podem ser impostas para a construção dos limitadores de fluxo. Isso é possível, pois Sweby [64] também transformou os conceitos introduzidos por Harten [26] em um conjunto de limitações para o comportamento dos limitadores de fluxo. Essas condições dão origem a região TVD no plano $\psi \perp r$ (Diagrama de Sweby) e são definidas por

$$
\begin{cases}0 \leq \psi(r) \leq \min (2 r, 2), & \text { se } r \geq 0 \\ \psi(r)=0, & \text { se } r<0\end{cases}
$$

A Figura 3.6 ilustra a região definida por (3.16). Segundo Sweby, se um esquema é TVD então seu limitador de fluxo deve estar contido inteiramente nessa região (a recíproca é verdadeira).

Outra propriedade proposta por Sweby [64] é o chamado princípio de monotonicidade de Sweby. Essa propriedade determina uma condição suficiente, porém não necessária, para que o limitador de fluxo preserve monotonicidade. Esta condição é definida por

$$
r \longrightarrow 0 \Longrightarrow \psi^{\prime}(r)=2
$$

Neste trabalho, o novo esquema desenvolvido possui uma relação funcional da forma (3.2) definida por uma função polinomial por partes em [0,1]. De acordo com Zijlema [81], usar funções suaves proporciona melhor comportamento com respeito a convergência em malhas grosseiras, o que justifica o desenvolvimento do novo esquema apresentado neste trabalho. 


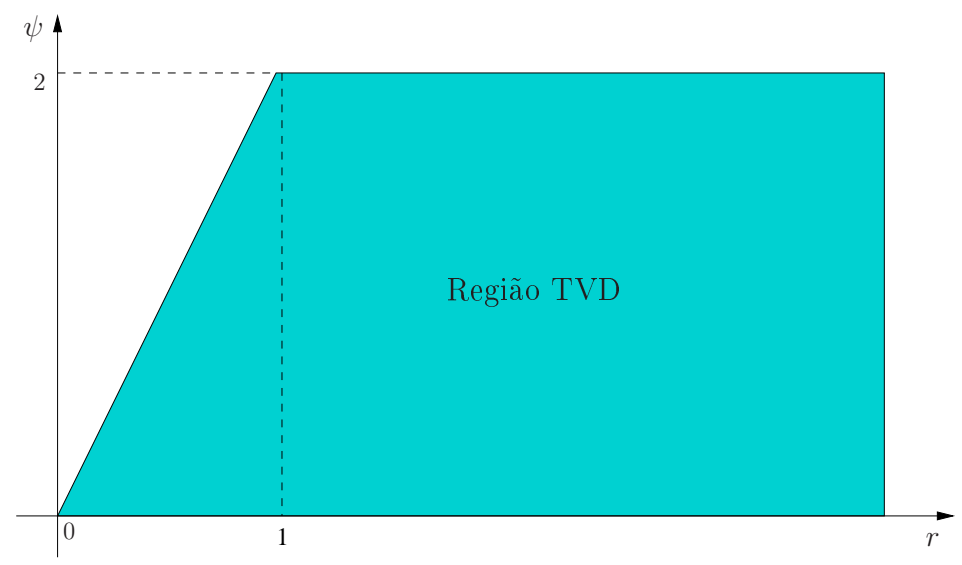

Figura 3.6: Região TVD - diagrama de Sweby.

Mais detalhes do desenvolvimento do novo esquema são discutidos no próximo capítulo.

\subsection{Esquemas convectivos relevantes}

Nesta seção são listados os esquemas upwind de alta resolução que são utilizados nesse trabalho.

\subsubsection{ADBQUICKEST}

O esquema denominado ADBQUICKEST (ADaptative Bounded Quadratic Upstream for Convective Kinemactics with Estimated Streaming Terms) foi desenvolvido por Ferreira et al. [20]. Em variáveis normalizadas é dado da forma

$$
\hat{\phi}_{f}= \begin{cases}(2-\theta) \hat{\phi}_{U}, & \text { se } 0 \leq \phi_{U}<a \\ \hat{\phi}_{U}+\frac{1}{2}(1-|\theta|)\left(1-\hat{\phi}_{U}\right)-\frac{1}{6}\left(1-\theta^{2}\right)\left(1-2 \hat{\phi}_{U}\right), & \text { se } a \leq \hat{\phi}_{U} \leq b, \\ 1-\theta+\theta \hat{\phi}_{U}, & \text { se } b<\hat{\phi}_{U} \leq 1 \\ \hat{\phi}_{U}, & \text { se } \hat{\phi}_{U} \notin[0,1]\end{cases}
$$

em que $a$ e $b$ são

$$
a=\frac{2-3|\theta|+\theta^{2}}{7-6 \theta-3|\theta|+2 \theta^{2}} \quad \text { e } \quad b=\frac{-4+6 \theta-3|\theta|+\theta^{2}}{-5+6 \theta-3|\theta|+2 \theta^{2}}
$$

$\operatorname{com} \theta$ o número de Courant. O limitador de fluxo correspondente desse esquema é

$$
\psi(r)=\max \left\{0, \min \left[2 r, \frac{2+\theta^{2}-3|\theta|+\left(1-\theta^{2}\right) r}{3-3|\theta|}, 2\right]\right\} .
$$




\subsubsection{TOPUS}

O esquema TOPUS (Third-Order Polynomial Upwind Scheme) foi desenvolvido por Queiroz [53] e, segundo o autor, ele pode ser visto como uma generalização do esquema SMARTER de Waterson e Deconinck [77]. Usando variáveis normalizadas, esse esquema é escrito como

$$
\hat{\phi}_{f}=\left\{\begin{array}{lll}
\left.\lambda \hat{\phi}_{U}^{4}+(-2 \lambda+1) \hat{\phi}_{U}^{3}+\left(\frac{5 \lambda-10}{4}\right) \hat{\phi}_{U}^{2}+\left(\frac{-\lambda+10}{4}\right) \hat{\phi}_{U}\right], & \text { se } & \hat{\phi}_{U} \in[0,1], \\
\hat{\phi}_{U}, & \text { se } & \hat{\phi}_{U} \notin[0,1],
\end{array}\right.
$$

com correspondente limitador de fluxo

$$
\psi(r)=\max \left\{0, \frac{0.5(|r|+r)\left[(-0.5 \lambda+1) r^{2}+(\lambda+4) r+(-0.5 \lambda+3)\right]}{(1+|r|)^{3}}\right\} .
$$

Os melhores resultados com esse esquema (ver [53]) são obtidos com $\lambda=2$, e portanto adota-se esse valor nas simulações realizadas neste trabalho.

\subsubsection{SDPUS-C1}

O esquema de classe $C^{1}$ denominado SDPUS-C1 (Six-Degree Polynomial Upwind Scheme of $C^{1}$ class) foi desenvolvido por Lima [15]. Sua representação em variáveis normalizadas é

$$
\hat{\phi}_{f}=\left\{\begin{array}{cl}
(-24+4 \gamma) \hat{\phi}_{U}^{6}+(68-12 \gamma) \hat{\phi}_{U}^{5}+(-64+13 \gamma) \hat{\phi}_{U}^{4}+ & \\
+(20-6 \gamma) \hat{\phi}_{U}^{3}+\gamma \hat{\phi}_{U}^{2}+\hat{\phi}_{U}, & \text { se } 0 \leq \hat{\phi}_{U} \leq 1, \\
\hat{\phi}_{U}, & \text { se } \quad \hat{\phi}_{U} \notin[0,1],
\end{array}\right.
$$

com limitador de fluxo dado pela expressão

$$
\psi(r)=\max \left\{0, \frac{0.5(|r|+r)\left[(-8+2 \gamma) r^{3}+(40-4 \gamma) r^{2}+2 \gamma r\right]}{(1+|r|)^{5}}\right\} .
$$

Para $\gamma \in[4,13]$ esse esquema está contido na região TVD e pode atingir terceira ordem de precisão. Os melhores resultados são alcançados com $\gamma=12$ (ver [15]) e, por isso, esse valor foi adotado nas simulações feitas nesse trabalho.

\subsubsection{WACEB}

O esquema de alta ordem WACEB (Weighted-Average Coefficient Ensuring Boundedness) foi desenvolvido por Song et al. [62]. Em variáveis normalizadas, ele é da forma

$$
\hat{\phi}_{f}= \begin{cases}2 \hat{\phi}_{U}, & \text { se } 0 \leq \hat{\phi}_{U}<3 / 10 \\ \frac{3}{4} \hat{\phi}_{U}+\frac{3}{8}, & \text { se } 3 / 10 \leq \hat{\phi}_{U} \leq 5 / 6 \\ 1, & \text { se } 5 / 6<\hat{\phi}_{U} \leq 1 \\ \hat{\phi}_{U}, & \text { se } \hat{\phi}_{U} \notin[0,1]\end{cases}
$$


cujo limitador de fluxo é definido por

$$
\psi(r)=\max \left\{0, \min \left[2 r, \frac{3}{4}+\frac{r}{4}, 2\right]\right\} .
$$

\subsection{5 van Albada}

Esse esquema foi proposto por van Albada et al. [73] e em variáveis normalizadas é dado como

$$
\hat{\phi}_{f}=\frac{\hat{\phi}_{U}\left(4 \hat{\phi}_{U}^{2}-5 \hat{\phi}_{U}+3\right)}{\left(4 \hat{\phi}_{U}^{2}-4 \hat{\phi}_{U}+2\right)} .
$$

Seu limitador de fluxo é

$$
\psi(r)=\frac{r(r+1)}{r^{2}+1}
$$

\subsubsection{Superbee}

O esquema de segunda ordem de precisão Superbee foi introduzido por Roe [57], e em variáveis normalizadas é da forma

$$
\hat{\phi}_{f}= \begin{cases}2 \hat{\phi}_{U}, & \text { se } 0 \leq \hat{\phi}_{U}<\frac{1}{3}, \\ \frac{1}{2}\left(1+\hat{\phi}_{U}\right), & \text { se } \frac{1}{3} \leq \hat{\phi}_{U}<\frac{1}{2} \\ \frac{3}{2} \hat{\phi}_{U}, & \text { se } \frac{1}{2} \leq \hat{\phi}_{U}<\frac{2}{3} \\ 1, & \text { se } \frac{2}{3} \leq \hat{\phi}_{U} \leq 1, \\ \hat{\phi}_{U}, & \text { se } \hat{\phi}_{U} \notin[0,1] .\end{cases}
$$

Expressando-o na forma de limitador de fluxo obtém-se

$$
\psi(r)=\max \{0, \min (1,2 r), \min (2, r)\}
$$

\subsubsection{MC}

O esquema popular MC (Monotonized Central-difference limiter) foi proposto por van Leer [39]. Seu limitador de fluxo é

$$
\psi(r)=\max \left\{0, \min \left[\frac{1+r}{2}, 2,2 r\right]\right\} .
$$




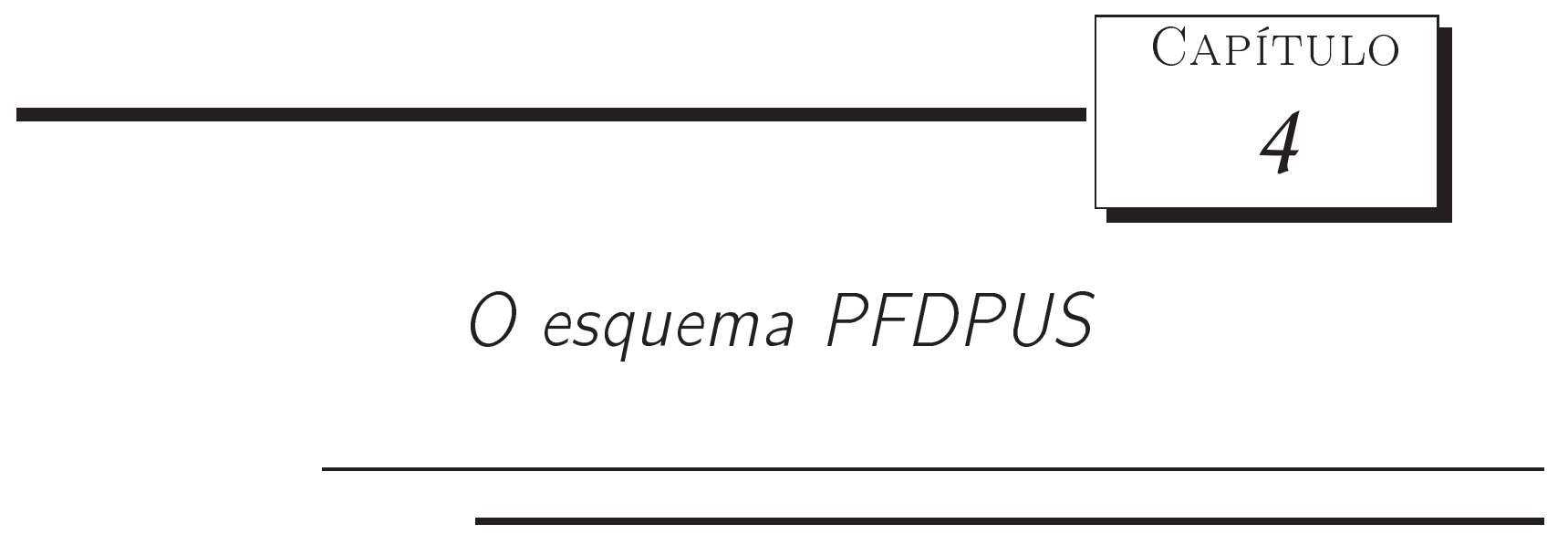

Neste capítulo apresenta-se o desenvolvimento do esquema upwind de alta resolução PFDPUS que é fruto desse trabalho.

\subsection{PFDPUS em variáveis normalizadas}

A derivação do esquema PFDPUS é baseada em uma função polinomial $\hat{\phi}_{f}\left(\hat{\phi}_{U}\right)$, definida no intervalo $[0,1]$, que é constituída de duas sentenças ( $\hat{\phi}_{f}$ é um polinômio por partes). Para derivar essas sentenças, consideram-se dois polinômios de grau quatro da forma

$$
\hat{\phi}_{f 1}\left(\hat{\phi}_{U}\right)=a_{4} \hat{\phi}_{U}^{4}+a_{3} \hat{\phi}_{U}^{3}+a_{2} \hat{\phi}_{U}^{2}+a_{1} \hat{\phi}_{U}+a_{0}, \quad \hat{\phi}_{U} \in\left[0, \frac{1}{2}\right]
$$

e

$$
\hat{\phi}_{f 2}\left(\hat{\phi}_{U}\right)=b_{4} \hat{\phi}_{U}^{4}+b_{3} \hat{\phi}_{U}^{3}+b_{2} \hat{\phi}_{U}^{2}+b_{1} \hat{\phi}_{U}+b_{0}, \quad \hat{\phi}_{U} \in\left[\frac{1}{2}, 1\right],
$$

onde são impostas as condições de Leonard [41] para monotonicidade e terceira ordem de precisão (ver Seção 3.1). Além disso, para evitar problemas em malhas grosseiras, impõe-se que a expressão global do esquema seja continuamente diferenciável em todo domínio computacional (ver Lin e Cheing [47]). Pode-se observar a partir de (4.1) e (4.2), que $\hat{\phi}_{f 1}$ e $\hat{\phi}_{f 2}$ estão definidos em $\hat{\phi}_{U}=\frac{1}{2}$, mas ao impor as condições de Leonard [41] estes polinômios tem valores iguais nesse ponto.

Formalmente, o novo esquema PFDPUS no intervalo [0,1] assume a forma

$$
\hat{\phi}_{f}=\left\{\begin{array}{lll}
\hat{\phi}_{f 1}, & \text { se } & \hat{\phi}_{U} \in\left[0, \frac{1}{2}\right), \\
\hat{\phi}_{f 2}, & \text { se } & \hat{\phi}_{U} \in\left[\frac{1}{2}, 1\right],
\end{array}\right.
$$


e como recomendado por Leonard [41], para $\hat{\phi}_{U} \notin[0,1]$, considera-se o esquema FOU [54] dado por

$$
\hat{\phi}_{f}=\hat{\phi}_{U}
$$

Para encontrar $\hat{\phi}_{f 1}$ deve-se determinar os coeficientes $a_{i}, i=0,1,2,3,4$, presentes na equação (4.1). Para tanto, o polinômio $\hat{\phi}_{f 1}$ deve:

- passar por $O=(0,0)$, o que implica $\hat{\phi}_{f 1}(0)=0$;

- passar por $Q=\left(\frac{1}{2}, \frac{3}{4}\right)$, o que implica $\hat{\phi}_{f 1}\left(\frac{1}{2}\right)=\frac{3}{4}$;

- passar por $Q$ com inclinação de $\frac{3}{4}$, o que implica $\hat{\phi}_{f 1}^{\prime}\left(\frac{1}{2}\right)=\frac{3}{4}$.

Além disso, impõe-se também, que $\hat{\phi}_{f}$ seja continuamente diferenciável em todo domínio, ou seja, $\hat{\phi}_{f 1}$ deve satisfazer

$$
\hat{\phi}_{f 1}^{\prime}(0)=1
$$

Como pode ser observado na expressão de $\hat{\phi}_{f 1}$, há cinco coeficientes que precisam ser determinados e há somente quatro condições impostas. Para fechar o sistema, o coeficiente $a_{2}=\alpha$ é escolhido como parâmetro livre. Essa escolha torna o esquema PFDPUS flexível, fazendo com que o usuário tenha a liberdade de escolher o parâmetro livre que melhor se adeque ao seu problema.

Impostas as condições acima, o sistema resultante é

$$
\left\{\begin{array}{l}
a_{0}=0 \\
a_{4}+2 a_{3}+8 a_{1}+16 a_{0}=12-4 \alpha \\
2 a_{4}+3 a_{3}+4 a_{1}=3-4 \alpha \\
a_{1}=1
\end{array}\right.
$$

e sua resolução acarreta

$$
a_{0}=0, \quad a_{1}=1, \quad a_{2}=\alpha, \quad a_{3}=9-4 \alpha \quad \text { e } \quad a_{4}=-14+4 \alpha .
$$

Substituindo esses coeficientes em (4.1), obtém-se

$$
\hat{\phi}_{f 1}\left(\hat{\phi}_{U}\right)=(-14+4 \alpha) \hat{\phi}_{U}^{4}+(9-4 \alpha) \hat{\phi}_{U}^{3}+\alpha \hat{\phi}_{U}^{2}+\hat{\phi}_{U}
$$

A determinação do polinômio $\hat{\phi}_{f 2}$ é feita de maneira análoga. Os coeficientes $b_{i}, i=$ $0,1,2,3,4$, de (4.2) devem ser encontrados impondo-se para $\hat{\phi}_{f 2}$ as condições

- passar por $Q=\left(\frac{1}{2}, \frac{3}{4}\right)$, o que implica $\hat{\phi}_{f 2}\left(\frac{1}{2}\right)=\frac{3}{4}$;

- passar por $Q$ com inclinação de $\frac{3}{4}$, o que implica $\hat{\phi}_{f 2}^{\prime}\left(\frac{1}{2}\right)=\frac{3}{4}$;

- passar por $P=(1,1)$, o que implica $\hat{\phi}_{f 2}(1)=1$. 
Em adição, $\hat{\phi}_{f}$ tem que ser continuamente diferenciável em todo domínio, o que implica

$$
\hat{\phi}_{f 2}^{\prime}(1)=1
$$

O sistema resultante após impor as condições acima e considerar o coeficiente $b_{2}=\beta$ como parâmetro livre é

$$
\left\{\begin{array}{l}
b_{4}+2 b_{3}+8 b_{1}+16 b_{0}=12-4 \beta \\
2 b_{4}+3 b_{3}+4 b_{1}=3-4 \beta \\
b_{4}+b_{3}+b_{1}+b_{0}=1-\beta \\
4 b_{4}+3 b_{3}+b_{1}=1-2 \beta
\end{array}\right.
$$

A solução do sistema (4.10) resulta

$$
b_{0}=\frac{1}{13} \beta, \quad b_{1}=2-\frac{6}{13} \beta, \quad b_{2}=\beta, \quad b_{3}=-3-\frac{12}{13} \beta \quad \text { e } \quad b_{4}=2+\frac{4}{13} \beta .
$$

Substituindo esses coeficientes em (4.2), segue

$$
\hat{\phi}_{f 2}\left(\hat{\phi}_{U}\right)=\left(2+\frac{4}{13} \beta\right) \hat{\phi}_{U}^{4}+\left(-3-\frac{12}{13} \beta\right) \hat{\phi}_{U}^{3}+\beta \hat{\phi}_{U}^{2}+\left(2-\frac{6}{13} \beta\right) \hat{\phi}_{U}+\left(\frac{1}{13} \beta\right) .
$$

Em resumo, o novo esquema convectivo PFDPUS em variáveis normalizadas para o cálculo dos fluxos numéricos nas interfaces das células computacionais é dado por

$$
\hat{\phi}_{f}= \begin{cases}(-14+4 \alpha) \hat{\phi}_{U}^{4}+(9-4 \alpha) \hat{\phi}_{U}^{3}+\alpha \hat{\phi}_{U}^{2}+\hat{\phi}_{U}, & \text { se } \hat{\phi}_{U} \in\left[0, \frac{1}{2}\right), \\ \left(2+\frac{4}{13} \beta\right) \hat{\phi}_{U}^{4}+\left(-3-\frac{12}{13} \beta\right) \hat{\phi}_{U}^{3}+\beta \hat{\phi}_{U}^{2}+\left(2-\frac{6}{13} \beta\right) \hat{\phi}_{U}+\left(\frac{1}{13} \beta\right), & \text { se } \quad \hat{\phi}_{U} \in\left[\frac{1}{2}, 1\right], \\ \hat{\phi}_{U}, & \text { se } \quad \hat{\phi}_{U} \notin[0,1] .\end{cases}
$$

A justificativa para denominar o novo esquema como PFDPUS (Piecewise Fourth Degree Polynomial Upwind Scheme), vem de que sua expressão global $\hat{\phi}_{f}$, em [0,1], é definida por um polinômio por partes, onde cada uma dessas partes é composta por um polinômio de grau quatro.

\subsection{PFDPUS em variáveis não normalizadas}

Para obter o esquema PFDPUS em variáveis não normalizadas, aplica-se a definição de variáveis normalizadas (3.3) em (4.13). Segue que:

$-\operatorname{para} \hat{\phi}_{U} \in\left[0, \frac{1}{2}\right)$

$$
\frac{\phi_{f}-\phi_{R}}{\phi_{D}-\phi_{R}}=(-14+4 \alpha) \hat{\phi}_{U}^{4}+(9-4 \alpha) \hat{\phi}_{U}^{3}+\alpha \hat{\phi}_{U}^{2}+\hat{\phi}_{U}
$$


o que implica em

$$
\phi_{f}=\phi_{R}+\left(\phi_{D}-\phi_{R}\right)\left[(-14+4 \alpha) \hat{\phi}_{U}^{4}+(9-4 \alpha) \hat{\phi}_{U}^{3}+\alpha \hat{\phi}_{U}^{2}+\hat{\phi}_{U}\right]
$$

$-\operatorname{para} \hat{\phi}_{U} \in\left[\frac{1}{2}, 1\right]$

$$
\frac{\phi_{f}-\phi_{R}}{\phi_{D}-\phi_{R}}=\left(2+\frac{4}{13} \beta\right) \hat{\phi}_{U}^{4}+\left(-3-\frac{12}{13} \beta\right) \hat{\phi}_{U}^{3}+\beta \hat{\phi}_{U}^{2}+\left(2-\frac{6}{13} \beta\right) \hat{\phi}_{U}+\left(\frac{1}{13} \beta\right),
$$

o que dá como resultado

$$
\phi_{f}=\phi_{R}+\left(\phi_{D}-\phi_{R}\right)\left[\left(2+\frac{4}{13} \beta\right) \hat{\phi}_{U}^{4}+\left(-3-\frac{12}{13} \beta\right) \hat{\phi}_{U}^{3}+\beta \hat{\phi}_{U}^{2}+\left(2-\frac{6}{13} \beta\right) \hat{\phi}_{U}+\left(\frac{1}{13} \beta\right)\right]
$$

$-\operatorname{para} \hat{\phi}_{U} \notin[0,1]$

$$
\frac{\phi_{f}-\phi_{R}}{\phi_{D}-\phi_{R}}=\frac{\phi_{U}-\phi_{R}}{\phi_{D}-\phi_{R}}
$$

o que resulta em

$$
\phi_{f}=\phi_{U}
$$

Resumindo, o esquema convectivo PFDPUS em variáveis não normalizadas tem a seguinte expressão

$$
\phi_{f}=\left\{\begin{array}{rr}
\phi_{R}+\left(\phi_{D}-\phi_{R}\right)\left[(-14+4 \alpha) \hat{\phi}_{U}^{4}+(9-4 \alpha) \hat{\phi}_{U}^{3}+\alpha \hat{\phi}_{U}^{2}+\hat{\phi}_{U}\right], & \text { se } \quad \hat{\phi}_{U} \in\left[0, \frac{1}{2}\right), \\
\phi_{R}+\left(\phi_{D}-\phi_{R}\right)\left[\left(2+\frac{4}{13} \beta\right) \hat{\phi}_{U}^{4}+\left(-3-\frac{12}{13} \beta\right) \hat{\phi}_{U}^{3}+\beta \hat{\phi}_{U}^{2}+\right. & \\
\left.+\left(2-\frac{6}{13} \beta\right) \hat{\phi}_{U}+\left(\frac{1}{13} \beta\right)\right], & \text { se } \quad \hat{\phi}_{U} \in\left[\frac{1}{2}, 1\right], \\
\phi_{U}, & \text { se } \hat{\phi}_{U} \notin[0,1] .
\end{array}\right.
$$

\subsection{Limitador de fluxo do esquema PFDPUS}

O limitador de fluxo correspondente ao esquema PFDPUS é obtido substituindo (4.13) na formulação de Waterson e Deconinck (3.10). Em seguida, a partir da relação (3.13), reescreve-se $\hat{\phi}_{U}$ em função de $r$, substituindo-o na expressão anteriormente conhecida. Com efeito:

$-\operatorname{para} \hat{\phi}_{U} \in\left[0, \frac{1}{2}\right)$

$$
(-14+4 \alpha) \hat{\phi}_{U}^{4}+(9-4 \alpha) \hat{\phi}_{U}^{3}+\alpha \hat{\phi}_{U}^{2}+\hat{\phi}_{U}=\hat{\phi}_{U}+\frac{1}{2} \psi(r)\left(1-\hat{\phi}_{U}\right),
$$


e fazendo as transformações matemáticas convenientes, segue

$$
\psi(r)=\frac{(2 \alpha-10) r^{4}+(18-4 \alpha) r^{3}+2 \alpha r^{2}}{(1+r)^{3}}
$$

$-\operatorname{para} \hat{\phi}_{U} \in\left[\frac{1}{2}, 1\right]$

$$
\left(2+\frac{4}{13} \beta\right) \hat{\phi}_{U}^{4}+\left(-3-\frac{12}{13} \beta\right) \hat{\phi}_{U}^{3}+\beta \hat{\phi}_{U}^{2}+\left(2-\frac{6}{13} \beta\right) \hat{\phi}_{U}+\left(\frac{1}{13} \beta\right)=\hat{\phi}_{U}+\frac{1}{2} \psi(r)\left(1-\hat{\phi}_{U}\right),
$$

que acarreta

$$
\psi(r)=\frac{\frac{1}{13}\left[(2 \beta+78) r^{2}+(26-4 \beta) r+2 \beta\right]}{(1+r)^{3}}
$$

$-\operatorname{para} \hat{\phi}_{U} \notin[0,1]$

$$
\hat{\phi}_{U}=\hat{\phi}_{U}+\frac{1}{2} \psi(r)\left(1-\hat{\phi}_{U}\right)
$$

o que dá como resultado

$$
\psi(r)=0
$$

Em resumo, o limitador de fluxo do esquema PFDPUS é

$$
\psi(r)= \begin{cases}\frac{(2 \alpha-10) r^{4}+(18-4 \alpha) r^{3}+2 \alpha r^{2}}{(1+r)^{3}}, & \text { se } \quad 0 \leq r<1, \\ \frac{\frac{1}{13}\left[(2 \beta+78) r^{2}+(26-4 \beta) r+2 \beta\right]}{(1+r)^{3}}, & \text { se } \quad r \geq 1, \\ 0, & \text { se } \quad r<0 .\end{cases}
$$

Para se evitar as condicionais na implementação computacional, as sentenças em (4.27) podem ser reescritas na forma

$$
\psi(r)=\frac{0.5(|r|+r)\left[(2 \alpha-10) r^{3}+(18-4 \alpha) r^{2}+2 \alpha r\right]}{(1+|r|)^{3}}, \quad \text { se } \quad 0 \leq r<1
$$

e

$$
\psi(r)=\frac{\frac{0.5(|r|+r)\left[(2 \beta+78) r+(26-4 \beta)+2 \beta r^{-1}\right]}{13}}{(1+|r|)^{3}}, \quad \text { se } \quad r \geq 1 .
$$

Na notação mais usual (ver LeVeque [43]), o limitador de fluxo de esquema PFDPUS é escrito como

$$
\psi(r)=\max \left\{0, \min \left[\frac{0.5(|r|+r)\left[(2 \alpha-10) r^{3}+(18-4 \alpha) r^{2}+2 \alpha r\right]}{(1+|r|)^{3}}, \frac{\frac{0.5(|r|+r)\left[(2 \beta+78) r+(26-4 \beta)+2 \beta r^{-1}\right]}{13}}{(1+|r|)^{3}}\right]\right\} .
$$


É importante ressaltar que o esquema PFDPUS é monotônico e de segunda ordem pois, para $r \geq 0$ e $\forall \alpha, \beta$, satisfaz (3.14). Com efeito,

$$
\psi(1)=\frac{\frac{1}{13}[(2 \beta+78)+(26-4 \beta)+2 \beta]}{8}=1 .
$$

Derivando a segunda sentença de (4.27), obtém-se

$$
\psi^{\prime}(r)=\frac{-2\left[\beta\left(r^{2}-6 r+5\right)+13\left(3 r^{2}-4 r-1\right)\right]}{13(r+1)^{4}},
$$

o que resulta em

$$
\psi^{\prime}(1)=\frac{-2[\beta(1-6+5)+13(3-4-1)]}{13(1+1)^{4}}=\frac{1}{4} .
$$

A partir desse resultado, pode-se constatar que o esquema PFDPUS pode atingir terceira ordem de precisão, uma vez que seu limitador de fluxo (4.27) satisfaz, para $r \geq 0$ e $\forall \alpha, \beta$, a condição (3.15) proposta por Zijlema [81].

Vale lembrar, que o limitador de fluxo do esquema PFDPUS não satisfaz o princípio de monotonicidade de Sweby (3.17), o que não implica que esse esquema não seja monotônico, uma vez que (3.17) é uma condição suficiente, porém não necessária para monotonicidade (essa propriedade é garantida pelas condições de Leonard [41]).

Em particular, o esquema PFDPUS é TVD para $\alpha \in[0,10]$ e para uma variedade de $\beta$ entre -38 e 100. Dentre estes, quatro combinações entre os parâmetros livres $\alpha$ e $\beta$ apresentam resultados satisfatórios nos testes numéricos realizados com as equações 1D de advecção e de Burgers e, então, são empregados nos testes numéricos apresentados nesse trabalho. As combinações são: $\alpha=0$ e $\beta=-38, \alpha=3$ e $\beta=100, \alpha=9$ e $\beta=88$, e, $\alpha=10$ e $\beta=100$. A forma como esses parâmetros livres foram encontrados pode ser vista no Apêndice A.

Para ilustrar que o esquema PFDPUS é TVD, a Figura 4.1 mostra este esquema nos planos $\hat{\phi}_{f} \perp \hat{\phi}_{U}$ e $\psi \perp r$ para as quatro combinações adotadas para $\alpha$ e $\beta$. 


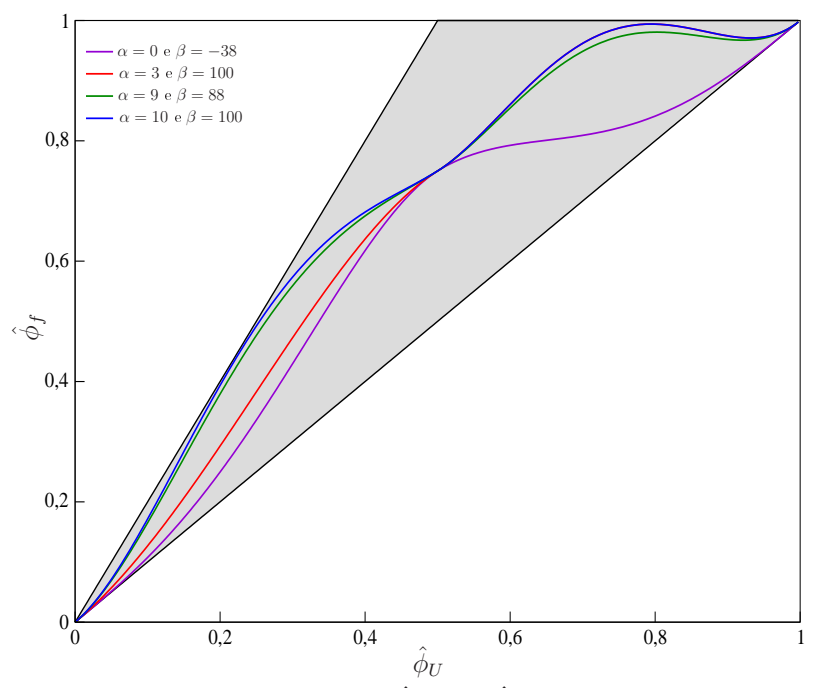

(a) $\hat{\phi}_{f} \perp \hat{\phi}_{U}$

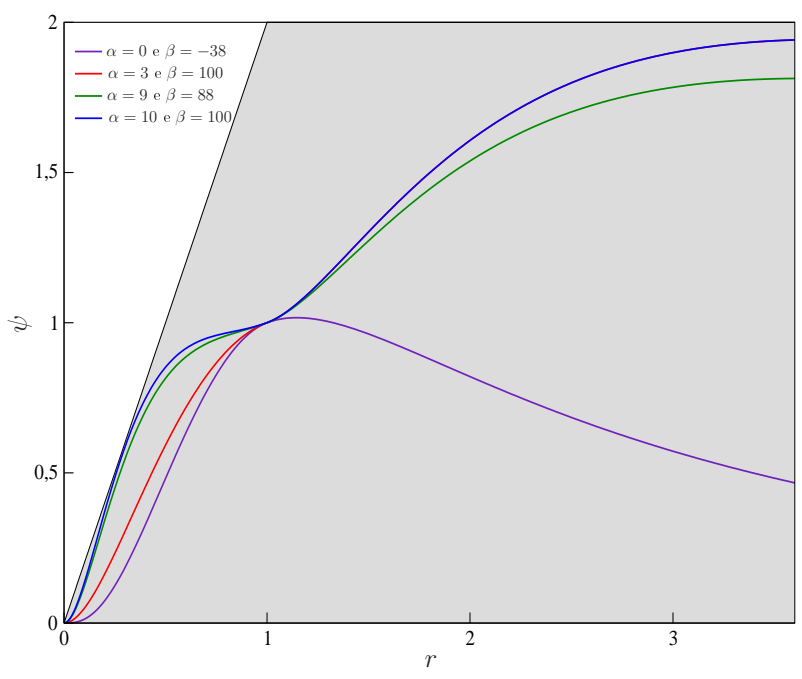

(b) $\psi \perp r$

Figura 4.1: Esquema PFDPUS na região TVD nos planos $\hat{\phi}_{f} \perp \hat{\phi}_{U}$ e $\psi \perp r$. 


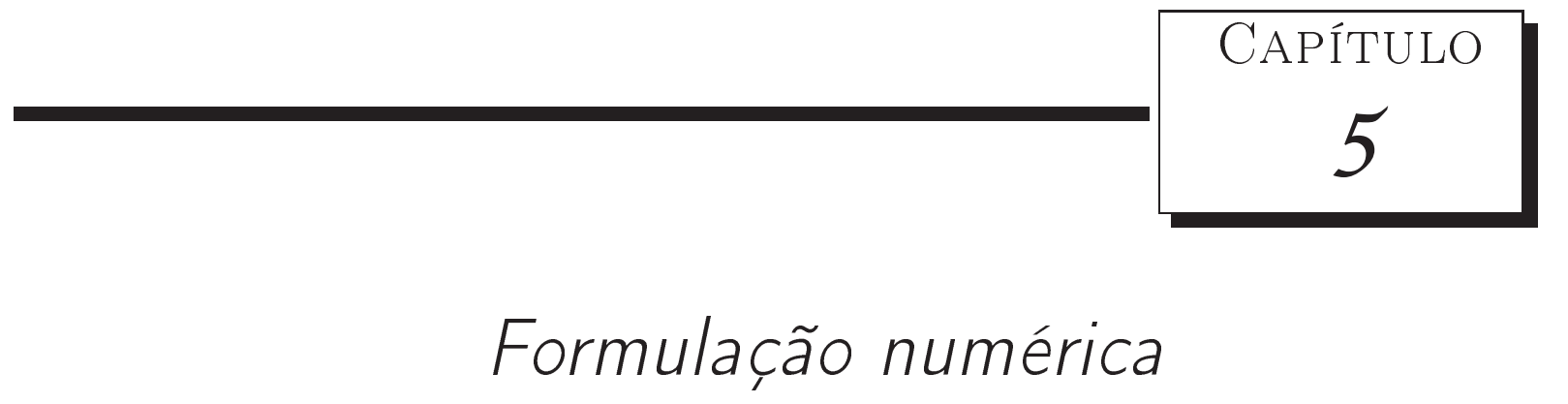

Este capítulo dá ênfase à discretização dos termos convectivos presentes nas leis de conservação 1D e 2D definidas, respectivamente, por (2.1) e (2.21); nas equações de Navier-Stokes (2.38), (2.39) e (2.36); e nas equações do modelo $\kappa-\varepsilon$ descritas por (2.50), (2.55) e (2.56) que modelam os efeitos da turbulência. Em seguida, apresentam-se os algoritmos base para a solução computacional de escoamentos incompressíveis com superfícies livres móveis laminares e turbulentos.

\subsection{Discretização das leis de conservação 1D}

Neste trabalho, as leis de conservação 1D (2.1), em particular as equações de advecção e de Burgers são aproximadas de duas formas. Uma das aproximações, onde considera-se o método de Euler explícito para a marcha no tempo, é dada da forma

$$
\phi_{i}^{n+1}=\phi_{i}^{n}-\frac{\delta t}{\delta x}\left(F(\phi)_{i+\frac{1}{2}}^{n}-F(\phi)_{i-\frac{1}{2}}^{n}\right)
$$

onde $\phi_{i}^{n}$ é a solução numérica no ponto de malha $(i, n) \equiv(i \delta x, n \delta t)$, sendo $\delta x$ e $\delta t$ os espaçamentos da malha (uniforme) nas direções $x$ e $t$, respectivamente. $\frac{\delta t}{\delta x}=\theta$ é chamado de número de Courant-Friedrichs-Lewy (CFL). Os termos $F(\phi)_{i+\frac{1}{2}}^{n}$ e $F(\phi)_{i-\frac{1}{2}}^{n}$ denotam, respectivamente, os fluxos numéricos nas interfaces $f=i+\frac{1}{2}$ e $g=i-\frac{1}{2}$ das células computacionais e são calculados através dos esquemas upwind de alta resolução.

No caso em que usa-se o método de Runge-Kutta TVD de terceira ordem (RK-3) (ver em 
[24] e [23]) para a discretização temporal, a aproximação é

$$
\begin{aligned}
\phi^{(1)} & =\phi_{i}^{n}-\frac{\delta t}{\delta x}\left(F(\phi)_{i+\frac{1}{2}}^{n}-F(\phi)_{i-\frac{1}{2}}^{n}\right) \\
\phi^{(2)} & =\frac{3}{4} \phi_{i}^{n}+\frac{1}{4} \phi^{(1)}-\frac{1}{4} \frac{\delta t}{\delta x}\left(F(\phi)_{i+\frac{1}{2}}^{(1)}-F(\phi)_{i-\frac{1}{2}}^{(1)}\right), \\
\phi_{i}^{n+1} & =\frac{1}{3} \phi_{i}^{n}+\frac{2}{3} \phi^{(2)}-\frac{2}{3} \frac{\delta t}{\delta x}\left(F(\phi)_{i+\frac{1}{2}}^{(2)}-F(\phi)_{i-\frac{1}{2}}^{(2)}\right),
\end{aligned}
$$

em que $\phi^{(1)}$ e $\phi^{(2)}$ são variáveis auxiliares para o cálculo final de $\phi_{i}^{n+1} \cdot \quad F(\phi)_{i+\frac{1}{2}}^{(1)}$ é o fluxo na interface $f$ calculado a partir dos esquemas upwind de alta resolução com o valor de $\phi^{(1)}$ previamente encontrado. As demais notações em (5.3) e (5.4) para os fluxos numéricos são análogas. Por simplicidade, o sobrescrito $n$ é omitido nas demais discretizações.

Os sistemas de equações hiperbólicas 1D de Euler e MHD são simulados com o auxílio do pacote computacional CLAWPACK ${ }^{1}$ (ver LeVeque [43]), equipado com os esquemas upwind de alta resolução estudados nesse trabalho. Mais detalhes das discretizações estão descritos abaixo.

\subsubsection{Equação de advecção 1D}

A equação de advecção tem variável conservada e função fluxo definidos por (2.3) e (2.4), respectivamente. Com efeito, no ponto $(i, n)$ da malha o fluxo é aproximado por

$$
F(\phi)_{i+\frac{1}{2}}-F(\phi)_{i-\frac{1}{2}}=\left.(a u)\right|_{i+\frac{1}{2}}-\left.(a u)\right|_{i-\frac{1}{2}}=a_{i+\frac{1}{2}} \cdot u_{i+\frac{1}{2}}-a_{i-\frac{1}{2}} \cdot u_{i-\frac{1}{2}}
$$

em que $a$ é a velocidade convectiva, que neste trabalho é considerada constante e igual a 1 em todo domínio computacional. As aproximações para $u_{i+\frac{1}{2}}$ e $u_{i-\frac{1}{2}}$ usando, por exemplo, o esquema PFDPUS são apresentadas na Seção 5.3 que trata da discretização das equações de Navier-Stokes 2D, 3D e axissimétricas.

\subsubsection{Equação de Burgers 1D - sem viscosidade}

Na equação de Burgers, a variável conservada e função fluxo são dados, respectivamente, por (2.8) e (2.9). Assim, o fluxo em $(i, n)$ é aproximado da forma

$$
F(\phi)_{i+\frac{1}{2}}-F(\phi)_{i-\frac{1}{2}}=\left.\left(\frac{u^{2}}{2}\right)\right|_{i+\frac{1}{2}}-\left.\left(\frac{u^{2}}{2}\right)\right|_{i-\frac{1}{2}}=\frac{1}{2}\left(\bar{u}_{i+\frac{1}{2}} \cdot u_{i+\frac{1}{2}}-\bar{u}_{i-\frac{1}{2}} \cdot u_{i-\frac{1}{2}}\right)
$$

onde as velocidades convectivas são calculadas como

$$
\bar{u}_{i-\frac{1}{2}}=\frac{1}{2}\left(u_{i}+u_{i-1}\right) \quad \text { e } \quad \bar{u}_{i+\frac{1}{2}}=\frac{1}{2}\left(u_{i+1}+u_{i}\right) .
$$

\footnotetext{
${ }^{1}$ http://www.amath.washington.edu/ claw/
} 
Da mesma forma como foi feito na discretização da equação de advecção 1D, as aproximações para $u_{i+\frac{1}{2}}$ e $u_{i-\frac{1}{2}}$ usando, por exemplo, o esquema PFDPUS são apresentadas na Seção 5.3.

\subsubsection{Equações de Euler 1D e MHD 1D}

Para as equações de Euler, o vetor de variáveis conservadas e função fluxo são dados, respectivamente, por (2.12) e (2.13). Já para as equações MHD, o vetor de variáveis conservadas e função fluxo são definidos por (2.16) e (2.17), respectivamente. As soluções numéricas para essas equações são calculadas usando-se o pacote computacional CLAWPACK (Conservation $L A W$ PACKage) de LeVeque [43] equipado com os limitadores de fluxo dos esquemas PFDPUS, ADBQUICKEST, TOPUS, SDPUS-C1, van Albada e Superbee. Esse pacote computacional simula leis de conservação hiperbólicas 1D, 2D e 3D fazendo o uso do método de volumes finitos. As soluções são computadas através do método de primeira ordem de Godunov [43] ou por sua variante de segunda ordem de precisão, o método de primeira ordem de Godunov com termo de correção [43]. Vale ressaltar que, a partir do método de primeira ordem de Godunov com termo de correção, é possível acrescentar no CLAWPACK uma variedade de métodos numéricos com pequenas modificações nas rotinas que implementam os limitadores de fluxo. Essas modificações têm por objetivo acrescentar os limitadores fluxos de outros esquemas desejados. Mais detalhes sobre o CLAWPACK e do algoritmo empregado para resolução do caso 1D pode ser encontrado no Apêndice B.

\subsection{Discretização das leis de conservação 2D}

As simulações dos sistemas hiperbólicos 2D de águas rasas (que tem vetor de variáveis conservadas (2.23) e funções fluxo (2.24) e (2.25), respectivamente), Euler (que tem vetor de variáveis conservadas (2.27) e funções fluxo (2.28) e (2.29), respectivamente) e MHD (que tem vetor de variáveis conservadas (2.32) e funções fluxo (2.33) e (2.34), respectivamente) são também simulados com o auxílio do pacote computacional CLAWPACK equipado com os limitadores de fluxo dos esquemas PFDPUS, ADBQUICKEST, TOPUS e SDPUS-C1. O método de solução utilizado no CLAWPACK pode ser encontrado no Apêndice B e mais detalhes para o caso 2D em LeVeque [43].

\subsection{Discretização das equações de Navier-Stokes}

As equações de Navier-Stokes 2D, 3D e axissimétricas para a simulação de escoamentos incompressíveis com superfícies livres móveis (com ou sem modelagem da turbulência) são discretizadas pelo método de diferenças finitas em uma malha deslocada (staggered grid) [2]. Nesse tipo de malha, as variáveis são armazenadas em posições diferentes, isto é, as velocidades são calculadas nas faces das células computacionais e os escalares como a pressão e as variáveis turbulentas $\kappa$, $\varepsilon$ e $\nu_{t}$ são aproximadas no centro da célula computacional. Aqui, todas as derivadas temporais são aproximadas pelo método de Euler explícito [21] e as derivadas espaciais 
por diferença central de segunda ordem de precisão.

O sistema discreto resultante é implementado no ambiente de simulação Freeflow de Castelo et al. [9], que é um sistema para modelagem, simulação e visualização de escoamentos incompressíveis com superfícies livres. Este ambiente computacional resolve as equações de transporte e continuidade baseado em uma variante do método da projeção, o método GENSMAC (Generalized Simplified Marker and Cell) de Tomé e MacKee [69]. Esse método é uma técnica de solução em diferenças finitas aplicada sobre uma malha deslocada que utiliza partículas marcadoras para a visualização do escoamento e localização da superfície livre do fluido.

A aproximação para os termos convectivos nas equações (2.38), (2.39), (2.36), (2.50), (2.55) e (2.56) é feita como segue. Por simplicidade, considera-se no caso 2D um termo convectivo típico avaliado num ponto $B$ da malha computacional da forma

$$
\left.\frac{\partial\left(u_{j} \phi\right)}{\partial\left(x_{j}\right)}\right|_{B}=\left.\frac{\partial(u \phi)}{\partial x}\right|_{B}+\left.\frac{\partial(v \phi)}{\partial y}\right|_{B},
$$

onde $\phi$ é a variável convectada (por exemplo $u, v, w, \kappa$ e $\varepsilon$ ) e $u_{j}$ a velocidade de convecção (que no caso da equação (5.8) é dado por $u$ e $v$ ). O ponto $B$ em (5.8) representa a posição em que o termo convectivo é avaliado.

Para exemplificar a aproximação, considera-se na equação (5.8) $\phi=u$ e $B=\left(i+\frac{1}{2}, j\right)$ (ver Figura 5.1). Nesse caso, o segundo termo do lado direito de (5.8) é aproximado como

$$
\left.\frac{\partial(v u)}{\partial y}\right|_{i+\frac{1}{2}, j}=\frac{v_{f} \cdot u_{i+\frac{1}{2}, j+\frac{1}{2}}-v_{g} \cdot u_{i+\frac{1}{2}, j-\frac{1}{2}}}{\delta y}
$$

onde $f=j+\frac{1}{2}$ e $g=j-\frac{1}{2}$. Os valores das velocidades convectivas $v_{f}$ e $v_{g}$ são estimados pelas médias

$$
\begin{aligned}
& v_{f}=v_{i+\frac{1}{2}, j+\frac{1}{2}} \approx \frac{v_{i+1, j+\frac{1}{2}}+v_{i, j+\frac{1}{2}}}{2}, \\
& v_{g}=v_{i+\frac{1}{2}, j-\frac{1}{2}} \approx \frac{v_{i+1, j-\frac{1}{2}}+v_{i, j-\frac{1}{2}}}{2},
\end{aligned}
$$

e os valores $u_{i+\frac{1}{2}, j+\frac{1}{2}}$ e $u_{i+\frac{1}{2}, j-\frac{1}{2}}$ são determinados por meio do esquema upwind de alta resolução PFDPUS em variáveis não normalizadas pela equação (4.20). Esses valores são interpolados usando-se os valores da propriedade convectada $u$ nos pontos vizinhos $D, U$ e $R$, os quais são automaticamente especificados de acordo com os sinais de $v_{f}$ e $v_{g}$ (ver Seção 3.1). Na sequência detalham-se as aproximações dos termos $u_{i+\frac{1}{2}, j+\frac{1}{2}}$ e $u_{i+\frac{1}{2}, j-\frac{1}{2}}$. 


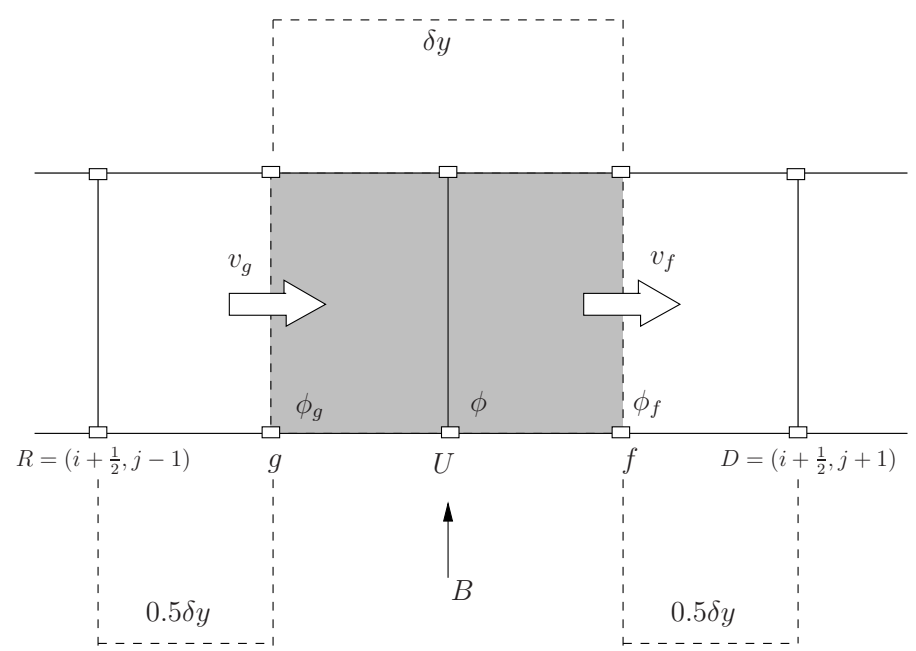

Figura 5.1: Célula computacional ilustrando o ponto $B$ de discretização dos termos convectivos, bem como, seus vizinhos, as faces $f$ e $g$ para a aproximação e a direção das velocidades $v_{f}$ e $v_{g}$ de convecção.

(1) Aproximações para $u_{i+\frac{1}{2}, j+\frac{1}{2}}$ :

- Quando $v_{f}=v_{i+\frac{1}{2}, j+\frac{1}{2}} \geq 0$, os pontos $D, U$ e $R$ ficam definidos como

$$
D=\left(i+\frac{1}{2}, j+1\right), \quad U=\left(i+\frac{1}{2}, j\right) \quad \text { e } \quad R=\left(i+\frac{1}{2}, j-1\right)
$$

e o termo convectivo $u_{i+\frac{1}{2}, j+\frac{1}{2}}$ é aproximado por

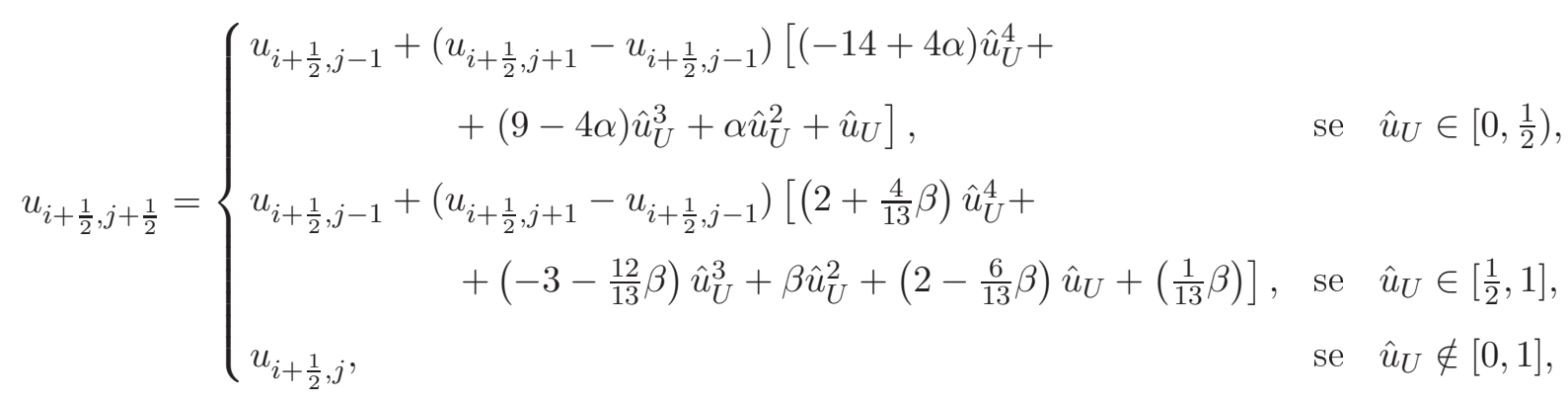

onde

$$
\hat{u}_{U}=\hat{u}_{i+\frac{1}{2}, j}=\frac{u_{i+\frac{1}{2}, j}-u_{i+\frac{1}{2}, j-1}}{u_{i+\frac{1}{2}, j+1}-u_{i+\frac{1}{2}, j-1}} ;
$$

- Quando $v_{f}=v_{i+\frac{1}{2}, j+\frac{1}{2}}<0$ os pontos $D, U$ e $R$ ficam definidos como

$$
D=\left(i+\frac{1}{2}, j\right), \quad U=\left(i+\frac{1}{2}, j+1\right) \quad \text { e } \quad R=\left(i+\frac{1}{2}, j+2\right)
$$


e o termo $u_{i+\frac{1}{2}, j+\frac{1}{2}}$ é aproximado por

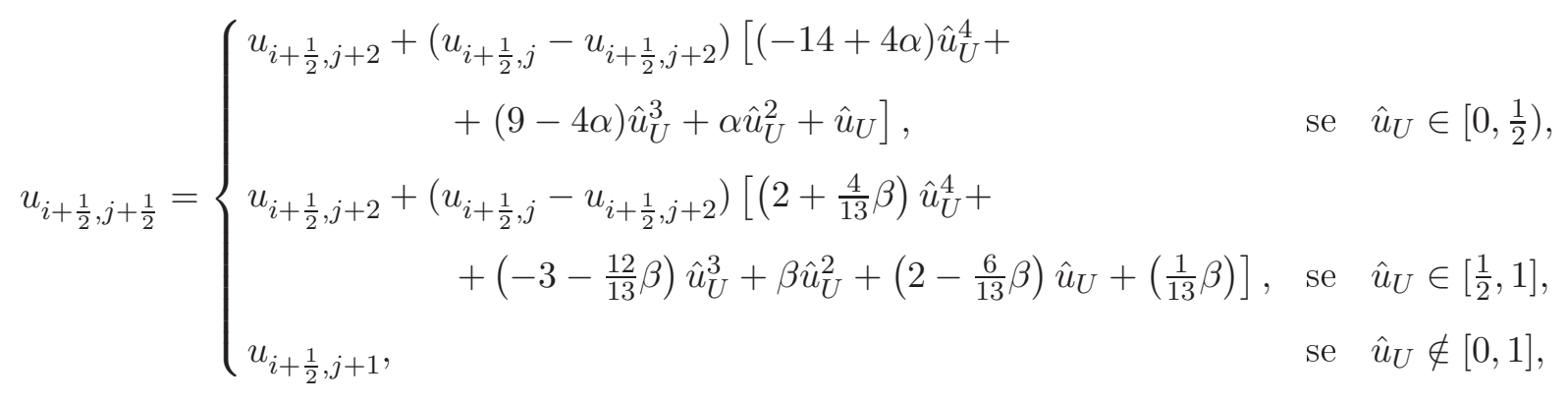

onde

$$
\hat{u}_{U}=\hat{u}_{i+\frac{1}{2}, j+1}=\frac{u_{i+\frac{1}{2}, j+1}-u_{i+\frac{1}{2}, j+2}}{u_{i+\frac{1}{2}, j}-u_{i+\frac{1}{2}, j+2}}
$$

(2) Aproximações para $u_{i+\frac{1}{2}, j-\frac{1}{2}}$ :

- Quando $v_{g}=v_{i+\frac{1}{2}, j-\frac{1}{2}} \geq 0$, os pontos $D, U$ e $R$ ficam definidos como

$$
D=\left(i+\frac{1}{2}, j\right), \quad U=\left(i+\frac{1}{2}, j-1\right) \quad \text { e } \quad R=\left(i+\frac{1}{2}, j-2\right)
$$

e o termo $u_{i+\frac{1}{2}, j-\frac{1}{2}}$ é aproximado por

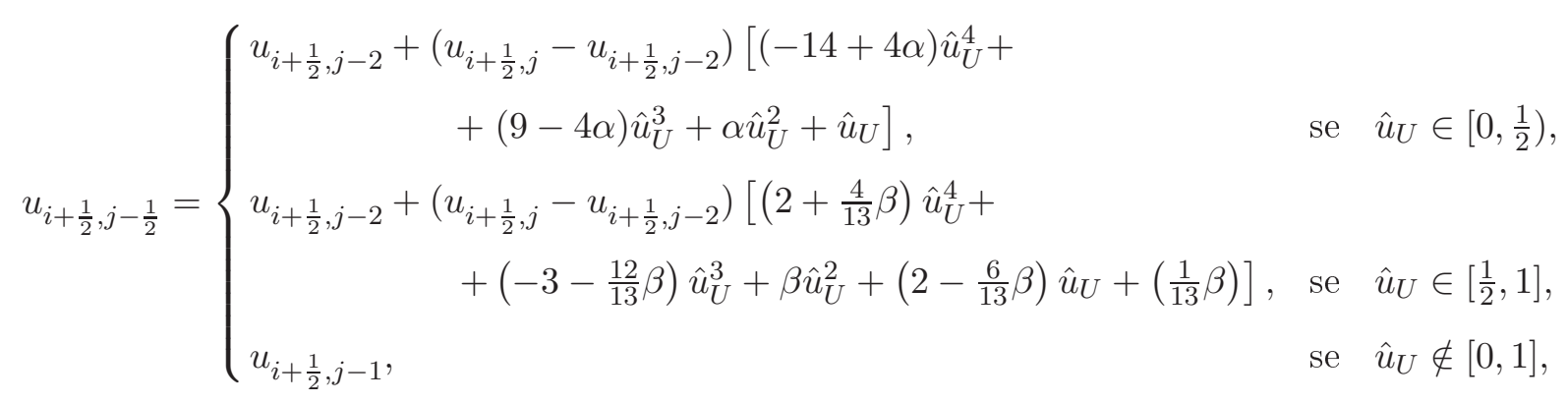

onde

$$
\hat{u}_{U}=\hat{u}_{i+\frac{1}{2}, j-1}=\frac{u_{i+\frac{1}{2}, j-1}-u_{i+\frac{1}{2}, j-2}}{u_{i+\frac{1}{2}, j}-u_{i+\frac{1}{2}, j-2}} ;
$$

- Quando $v_{g}=v_{i+\frac{1}{2}, j-\frac{1}{2}}<0$, os pontos $D, U$ e $R$ ficam definidos como

$$
D=\left(i+\frac{1}{2}, j-1\right), \quad U=\left(i+\frac{1}{2}, j\right) \quad \text { e } \quad R=\left(i+\frac{1}{2}, j+1\right)
$$

e o termo $u_{i+\frac{1}{2}, j-\frac{1}{2}}$ é aproximado por

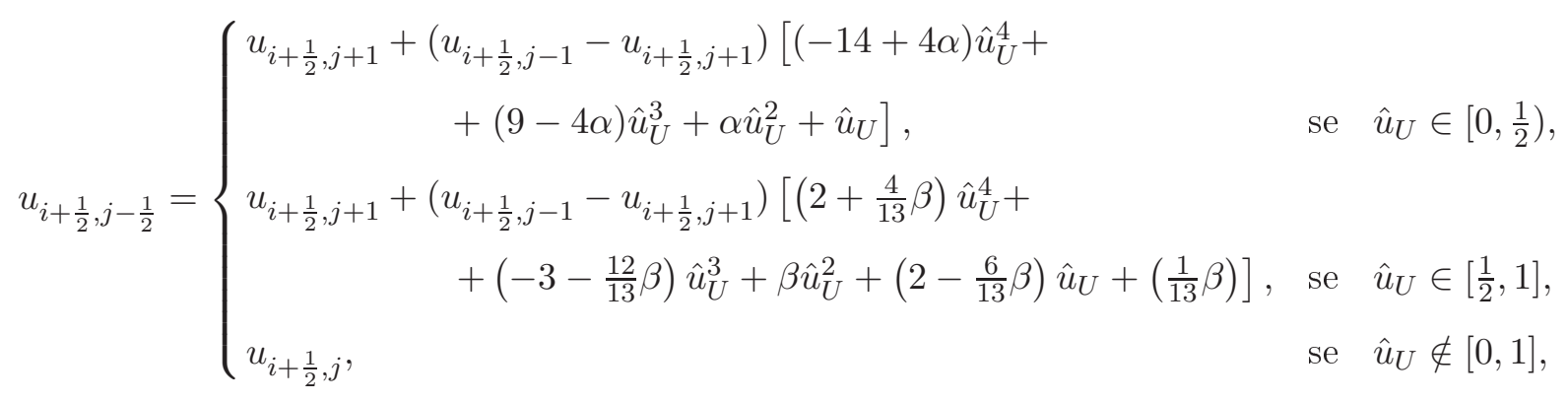


onde

$$
\hat{u}_{U}=\hat{u}_{i+\frac{1}{2}, j}=\frac{u_{i+\frac{1}{2}, j}-u_{i+\frac{1}{2}, j+1}}{u_{i+\frac{1}{2}, j-1}-u_{i+\frac{1}{2}, j+1}} .
$$

A aproximação upwind, usando o esquema PFDPUS, para o primeiro termo do lado direito da equação (5.8) é feita de maneira análoga.

Considera-se agora a aproximação upwind para o caso de uma propriedade convectada $\phi$ no centro da célula computacional. Por exemplo, considera-se $\phi$ como sendo a variável turbulenta $\kappa$ (energia cinética da turbulência) e a velocidade de convecção como sendo $u$ na direção $x$, ou seja, $\phi=\kappa$ no primeiro termo do lado direito da equação (5.8). Nesse caso, $B=(i, j)$ e a derivada convectiva correspondente é aproximada por

$$
\left.\frac{\partial(u \kappa)}{\partial x}\right|_{B}=\frac{\left.(u \kappa)\right|_{f}-\left.(u \kappa)\right|_{g}}{\delta x}
$$

onde $f=i+\frac{1}{2}$ e $g=i-\frac{1}{2}$. Assim, a equação (5.12) torna-se

$$
\left.\frac{\partial(u \kappa)}{\partial x}\right|_{i, j}=\frac{u_{f} \cdot \kappa_{i+\frac{1}{2}, j}-u_{g} \cdot \kappa_{i-\frac{1}{2}, j}}{\delta x},
$$

onde as velocidades convectivas $u_{f}$ e $u_{g}$ são

$$
\begin{aligned}
& u_{f}=u_{i+\frac{1}{2}, j}, \\
& u_{g}=u_{i-\frac{1}{2}, j} .
\end{aligned}
$$

As aproximações para $\kappa_{i+\frac{1}{2}, j}$ e $\kappa_{i-\frac{1}{2}, j}$ em (5.13) são feitas utilizando o esquema upwind de alta resolução PFDPUS em variáveis não normalizadas como segue:

(1) Aproximações para $\kappa_{i+\frac{1}{2}, j}$ :

- Quando $u_{f}=u_{i+\frac{1}{2}, j} \geq 0$, os pontos $D, U$ e $R$ ficam definidos como

$$
D=(i+1, j), \quad U=(i, j) \quad \text { e } \quad R=(i-1, j)
$$

e o termo $\kappa_{i+\frac{1}{2}, j}$ é aproximado por

$$
\kappa_{i+\frac{1}{2}, j}= \begin{cases}\kappa_{i-1, j}+\left(\kappa_{i+1, j}-\kappa_{i-1, j}\right)\left[(-14+4 \alpha) \hat{\kappa}_{U}^{4}+(9-4 \alpha) \hat{\kappa}_{U}^{3}+\alpha \hat{\kappa}_{U}^{2}+\hat{\kappa}_{U}\right], & \text { se } \hat{\kappa}_{U} \in\left[0, \frac{1}{2}\right), \\ \kappa_{i-1, j}+\left(\kappa_{i+1, j}-\kappa_{i-1, j}\right)\left[\left(2+\frac{4}{13} \beta\right) \hat{\kappa}_{U}^{4}+\right. & \\ \left.\quad+\left(-3-\frac{12}{13} \beta\right) \hat{\kappa}_{U}^{3}+\beta \hat{\kappa}_{U}^{2}+\left(2-\frac{6}{13} \beta\right) \hat{\kappa}_{U}+\left(\frac{1}{13} \beta\right)\right], & \text { se } \hat{\kappa}_{U} \in\left[\frac{1}{2}, 1\right], \\ \kappa_{i, j}, & \text { se } \hat{\kappa}_{U} \notin[0,1],\end{cases}
$$

onde

$$
\hat{\kappa}_{U}=\hat{\kappa}_{i, j}=\frac{\kappa_{i, j}-\kappa_{i-1, j}}{\kappa_{i+1, j}-\kappa_{i-1, j}}
$$

- Quando $u_{f}=u_{i+\frac{1}{2}, j}<0$, os pontos $D, U$ e $R$ ficam definidos como 


$$
D=(i, j), \quad U=(i+1, j) \quad \text { e } \quad R=(i+2, j)
$$

e o termo $\kappa_{i+\frac{1}{2}, j}$ é aproximado por

$$
\kappa_{i+\frac{1}{2}, j}=\left\{\begin{aligned}
\kappa_{i+2, j}+\left(\kappa_{i, j}-\kappa_{i+2, j}\right)\left[(-14+4 \alpha) \hat{\kappa}_{U}^{4}+(9-4 \alpha) \hat{\kappa}_{U}^{3}+\alpha \hat{\kappa}_{U}^{2}+\hat{\kappa}_{U}\right], & \text { se } \hat{\kappa}_{U} \in\left[0, \frac{1}{2}\right), \\
\kappa_{i+2, j}+\left(\kappa_{i, j}-\kappa_{i+2, j}\right)\left[\left(2+\frac{4}{13} \beta\right) \hat{\kappa}_{U}^{4}+\right. & \\
\left.+\left(-3-\frac{12}{13} \beta\right) \hat{\kappa}_{U}^{3}+\beta \hat{\kappa}_{U}^{2}+\left(2-\frac{6}{13} \beta\right) \hat{\kappa}_{U}+\left(\frac{1}{13} \beta\right)\right], & \text { se } \quad \hat{\kappa}_{U} \in\left[\frac{1}{2}, 1\right], \\
\kappa_{i+1, j}, & \text { se } \quad \hat{\kappa}_{U} \notin[0,1],
\end{aligned}\right.
$$

onde

$$
\hat{\kappa}_{U}=\hat{\kappa}_{i+1, j}=\frac{\kappa_{i+1, j}-\kappa_{i+2, j}}{\kappa_{i, j}-\kappa_{i+2, j}}
$$

(2) Aproximações para $\kappa_{i-\frac{1}{2}, j}$ :

- Quando $u_{g}=u_{i-\frac{1}{2}, j} \geq 0$, os pontos $D, U$ e $R$ ficam definidos como

$$
D=(i, j), \quad U=(i-1, j) \quad \text { e } \quad R=(i-2, j)
$$

e o termo $\kappa_{i-\frac{1}{2}, j}$ é aproximado por

$$
\kappa_{i-\frac{1}{2}, j}=\left\{\begin{aligned}
\kappa_{i-2, j}+\left(\kappa_{i, j}-\kappa_{i-2, j}\right)\left[(-14+4 \alpha) \hat{\kappa}_{U}^{4}+(9-4 \alpha) \hat{\kappa}_{U}^{3}+\alpha \hat{\kappa}_{U}^{2}+\hat{\kappa}_{U}\right], & \text { se } \hat{\kappa}_{U} \in\left[0, \frac{1}{2}\right), \\
\kappa_{i-2, j}+\left(\kappa_{i, j}-\kappa_{i-2, j}\right)\left[\left(2+\frac{4}{13} \beta\right) \hat{\kappa}_{U}^{4}+\right. & \\
\left.+\left(-3-\frac{12}{13} \beta\right) \hat{\kappa}_{U}^{3}+\beta \hat{\kappa}_{U}^{2}+\left(2-\frac{6}{13} \beta\right) \hat{\kappa}_{U}+\left(\frac{1}{13} \beta\right)\right], & \text { se } \quad \hat{\kappa}_{U} \in\left[\frac{1}{2}, 1\right], \\
\kappa_{i-1, j}, & \text { se } \quad \hat{\kappa}_{U} \notin[0,1],
\end{aligned}\right.
$$

em que

$$
\hat{\kappa}_{U}=\hat{\kappa}_{i-1, j}=\frac{\kappa_{i-1, j}-\kappa_{i-2, j}}{\kappa_{i, j}-\kappa_{i-2, j}}
$$

- Quando $u_{g}=u_{i-\frac{1}{2}, j}<0$, os pontos $D, U$ e $R$ ficam definidos como

$$
D=(i-1, j), \quad U=(i, j) \quad \text { e } \quad R=(i+1, j)
$$

e o termo $\kappa_{i-\frac{1}{2}, j}$ é aproximado por

$$
\kappa_{i-\frac{1}{2}, j}= \begin{cases}\kappa_{i+1, j}+\left(\kappa_{i-1, j}-\kappa_{i+1, j}\right)\left[(-14+4 \alpha) \hat{\kappa}_{U}^{4}+(9-4 \alpha) \hat{\kappa}_{U}^{3}+\alpha \hat{\kappa}_{U}^{2}+\hat{\kappa}_{U}\right], & \text { se } \hat{\kappa}_{U} \in\left[0, \frac{1}{2}\right), \\ \kappa_{i+1, j}+\left(\kappa_{i-1, j}-\kappa_{i+1, j}\right)\left[\left(2+\frac{4}{13} \beta\right) \hat{\kappa}_{U}^{4}+\right. & \\ \left.\quad+\left(-3-\frac{12}{13} \beta\right) \hat{\kappa}_{U}^{3}+\beta \hat{\kappa}_{U}^{2}+\left(2-\frac{6}{13} \beta\right) \hat{\kappa}_{U}+\left(\frac{1}{13} \beta\right)\right], & \text { se } \hat{\kappa}_{U} \in\left[\frac{1}{2}, 1\right], \\ \kappa_{i, j}, & \text { se } \quad \hat{\kappa}_{U} \notin[0,1],\end{cases}
$$

onde

$$
\hat{\kappa}_{U}=\hat{\kappa}_{i, j}=\frac{\kappa_{i, j}-\kappa_{i+1, j}}{\kappa_{i-1, j}-\kappa_{i+1, j}}
$$


Pode-se notar que o esquema PFDPUS utiliza três pontos vizinhos $D, U$ e $R$ para aproximar as derivadas. Dessa forma, podem surgir pontos fora do domínio de solução para as células computacionais próximas às fronteiras. Quando isso ocorre, o esquema híbrido (uma combinação do esquema FOU e diferenças centradas) (ver [21]) é empregado. Para simular escoamentos com superfícies livres móveis, os parâmetros livres $\alpha$ e $\beta$ do esquema PFDPUS são adotados como 10 e 100, respectivamente.

\subsubsection{Algoritmo computacional}

Nesta subseção é apresentado o algoritmo computacional para simular escoamentos incompressíveis com superfícies livres móveis com e sem a modelagem da turbulência.

Para simular escoamentos de fluidos sem a modelagem da turbulência, considera-se que num dado instante de tempo $t_{0}\left(\right.$ ou $t_{n}$ ) as variáveis dependentes são conhecidas (as condições iniciais) e as condições de contorno para a velocidade e pressão estão especificadas. Um ciclo computacional consiste na atualização das variáveis discretas a partir de um tempo inicial $t_{0}$ (ou um tempo anterior $t_{n}$ ) no tempo $t_{1}=t_{0}+\delta t$ (ou $t_{n+1}=t_{n}+\delta t$ ) usando-se uma série de passos inter-relacionados, que neste trabalho, são baseados no método GENSMAC [69]. Dessa forma, o campo de velocidade atualizado é calculado através dos seguintes passos:

Passo 1: Atualizam-se as condições de contorno nas regiões de entrada e saída de fluido, e nas paredes rígidas. Calcula-se o campo de velocidade na superfície livre por meio das equações (2.45) e (2.46). A pressão na superfície livre é calculada usando-se a equação (2.47) [69];

Passo 2: Determina-se um campo de velocidade intermediário $\tilde{u}_{i}$ por meio de

$$
\tilde{u}_{i}=u_{i}+\delta t\left\{-\frac{\partial\left(u_{i} u_{j}\right)}{\partial x_{j}}-\frac{\partial \tilde{P}}{\partial x_{i}}+\frac{1}{R e} \frac{\partial}{\partial x_{j}}\left(\frac{\partial u_{i}}{\partial x_{j}}\right)+\frac{1}{F r^{2}} g_{i}\right\} \quad i=1,2,3,
$$

em que $\tilde{P}$ é uma pressão arbitrária (tentativa);

Passo 3: Resolve-se a equação de Poisson para o potencial auxiliar $\varphi$ através de

$$
\frac{\partial}{\partial x_{i}}\left(\frac{\partial \varphi}{\partial x_{i}}\right)=\left(\frac{\partial \tilde{u}_{i}}{\partial x_{i}}\right), \quad i=1,2,3,
$$

com a condição homogênea de Dirichlet $(\varphi=0)$ na superfície livre e saída, e com condição homogênea de Neumann $\left(\frac{\partial \varphi}{\partial n}=0\right)$ nos contornos rígidos e na entrada. Para resolver o sistema de equação lineares resultante, o método dos gradientes conjugados [28] é utilizado;

Passo 4: Atualiza-se o campo de velocidade por (ver detalhes em Denaro [17])

$$
u_{i}=\tilde{u}_{i}-\frac{\partial \varphi}{\partial x_{i}}, \quad i=1,2,3
$$


Passo 5: Atualiza-se a pressão como (ver detalhes em Tomé e McKee [69])

$$
P=\tilde{P}+\frac{\varphi}{\delta t}
$$

Passo 6: Determina-se as novas posições das partículas marcadoras que representam o fluido. Essas partículas permitem a visualização do escoamento e a orientação da superfície livre. As posições são atualizadas resolvendo

$$
\frac{d x_{i}}{d t}=u_{i}, \quad i=1,2,3
$$

pelo método de Euler explícito [21].

Por fim, retorna-se ao passo inicial dando início ao novo ciclo computacional.

Para descrever o algoritmo base para a simulação de escoamentos incompressíveis turbulentos e calcular as variáveis $u, v, \kappa$ e $\varepsilon$ de (2.50), (2.51), (2.55) e (2.56), também são consideradas que no instante $t_{0}$ as variáveis dependentes são conhecidas e as condições de fronteira associadas estão especificadas. As equações médias de Reynolds e as equações que descrevem as características da turbulência são resolvidas de maneira desacoplada (método de projeção de Chorin [10]) como foi feito na seção anterior: primeiramente as equações médias do movimento e continuidade são resolvidas, mantendo-se todas as grandezas turbulentas "congeladas"; em seguida, resolve-se as equações para as variáveis turbulentas. A sequência de passos é descrita como segue:

Passo 1: Com a viscosidade turbulenta $\nu_{t}$ conhecida no tempo inicial $t_{0}$, ou num ciclo prévio, computa-se um campo de velocidade tentativo $\tilde{u}_{i}$ em $t_{1}=t_{0}+\delta t$ da forma

$$
\tilde{u}_{i}=u_{i}+\delta t\left\{-\frac{\partial\left(u_{i} u_{j}\right)}{\partial x_{j}}-\frac{\partial \tilde{P}}{\partial x_{i}}+\frac{1}{R e} \frac{\partial}{\partial x_{j}}\left(\frac{\partial u_{i}}{\partial x_{j}}\right)+\frac{1}{F r^{2}} g_{i}-\frac{1}{R e} \frac{\partial\left(\overline{u_{i} u_{j}}\right)}{\partial x_{j}}\right\}, \quad i=1,2
$$

O tensor de tensões $\overline{u_{i} u_{j}}$ é calculado pelo modelo $\kappa-\varepsilon$;

Passo 2: Resolve-se a equação de Poisson para o potencial $\varphi$ da forma

$$
\frac{\partial}{\partial x_{i}}\left(\frac{\partial \varphi}{\partial x_{i}}\right)=\left(\frac{\partial \tilde{u}_{i}}{\partial x_{i}}\right), \quad i=1,2
$$

adotando-se as mesmas condições do passo 3 acima descrito;

Passo 3: Atualiza-se o campo de velocidade por

$$
u_{i}=\tilde{u}_{i}-\frac{\partial \varphi}{\partial x_{i}}, \quad i=1,2
$$

Passo 4: Atualiza-se a pressão da forma

$$
P=\tilde{P}+\frac{\varphi}{\delta t}
$$


Passo 5: Calcula-se a energia cinética turbulenta $\kappa$ a partir de

$$
\kappa^{n+1}=\kappa^{n}+\delta t\left\{-\frac{\partial\left(\kappa u_{j}\right)}{\partial x_{j}}+\frac{1}{R e} \frac{\partial}{\partial x_{j}}\left[\left(1+\frac{\nu_{t}}{\sigma_{\kappa}}\right) \frac{\partial \kappa}{\partial x_{j}}\right]+\nu_{t} D_{i j} \frac{\partial u_{i}}{\partial x_{j}}-\varepsilon\right\}, \quad i=1,2
$$

onde $D_{i j}$ é dado por (2.53) e $n+1$ denota o passo de tempo atual;

Passo 6: Calcula-se a dissipação de energia $\varepsilon$ a partir de

$$
\varepsilon^{n+1}=\varepsilon^{n}+\delta t\left\{-\frac{\partial\left(\varepsilon u_{j}\right)}{\partial x_{j}}+\frac{1}{R e} \frac{\partial}{\partial x_{j}}\left[\left(1+\frac{\nu_{t}}{\sigma_{\varepsilon}}\right) \frac{\partial \varepsilon}{\partial x_{j}}\right]+\frac{\varepsilon}{\kappa}\left(C_{1 \varepsilon} \nu_{t} D_{i j} \frac{\partial u_{i}}{\partial x_{j}}-C_{2 \varepsilon} \varepsilon\right)\right\}, \quad i=1,2 ;
$$

Passo 7: Calcula-se a viscosidade turbulenta $\nu_{t}$ através de

$$
\nu_{t}=C_{\mu} \frac{\kappa^{2}}{\varepsilon}
$$

Passo 8: Atualiza-se a posição das partículas marcadoras que representam o fluido por meio da aplicação do método de Euler [21] ao sistema de equações diferenciais ordinárias (EDOs)

$$
\frac{d x_{i}}{d t}=u_{i}, \quad i=1,2
$$

Passo 9: Atualizam-se as condições de contorno necessárias para o próximo ciclo computacional.

Mais detalhes sobre algoritmo computacional para simulação de escoamentos incompressíveis com superfícies livres podem ser encontrados em Kurokawa [38], Brandi [6] e Bambozzi $[16]$. 


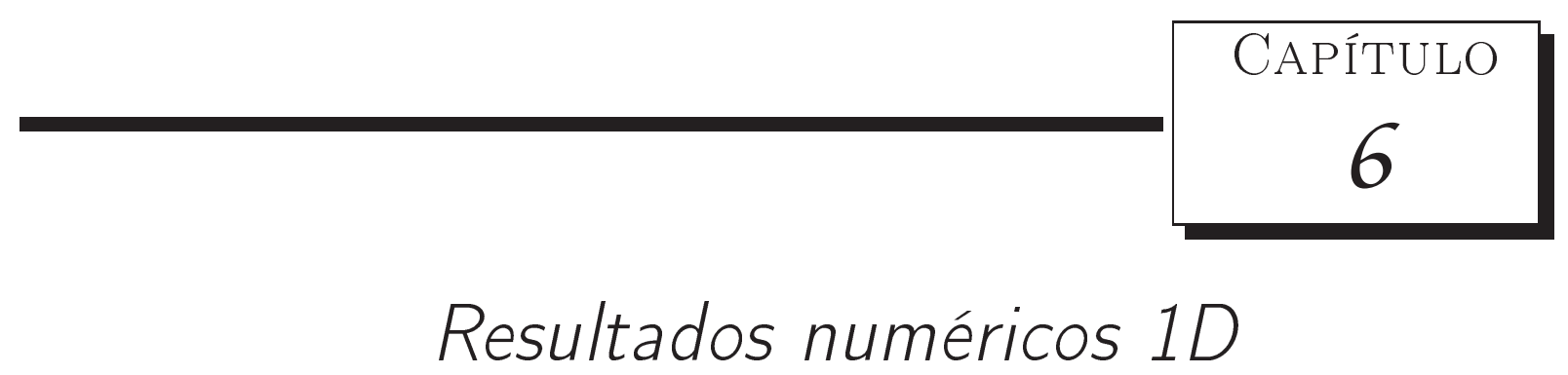

Este capítulo relata os experimentos numéricos realizados para as leis de conservação 1D presentes no Capítulo 2, bem como, as observações e verificações feitas com base nos resultados numéricos obtidos nestes experimentos. Em particular, resolve-se uma variedade de problemas modelados pelas equações de advecção, Burgers sem viscosidade, Euler e MHD. O objetivo é analisar o comportamento do esquema PFDPUS e determinar para quais pares de parâmetros livres $\alpha$ e $\beta$ os melhores resultados são obtidos, e, a partir daí, constatar que seu desempenho pode ser comparado com os esquemas presentes no estado da arte atual.

\subsection{Equação de advecção}

Aqui, são apresentados os resultados numéricos obtidos pela lei de conservação 1D (2.1), que tem variável conservada (2.3) e fluxo numérico (2.4). São realizados testes para três condições iniciais diferentes, uma suave e duas com descontinuidades na solução inicial. Nos testes, as soluções numéricas com esquema PFDPUS são obtidas pela combinação dos parâmetros $\alpha$ e $\beta$ (isto é, $\alpha=0$ e $\beta=-38, \alpha=3$ e $\beta=100, \alpha=9$ e $\beta=88$, e, $\alpha=10$ e $\beta=$ 100). Essas soluções são confrontadas com as soluções exatas e as soluções numéricas geradas pelos esquemas ADBQUICKEST, TOPUS, SDPUS-C1, WACEB e van Albada. Além disso, analisa-se a restrição TVD e calcula-se estimativas para a ordem de convergência. O avanço temporal é feito usando Euler explícito (ver (5.1)) e o método de Runge-Kutta TVD de terceira ordem (RK-3)(ver (5.2)-(5.4)). 


\section{Problema 1: (Condição inicial suave)}

Este teste é selecionado para checar a ordem de convergência na norma $L_{1}$ para cada um dos esquemas considerados nessa seção. A condição inicial é dada da forma

$$
u(x, 0)=\operatorname{sen}(\pi x)
$$

O domínio computacional é $[-1,1]$ e as condições de contorno são periódicas. Para a simulação são consideradas malhas de 20,40, 80, 160, 320 e 640 células computacionais, $\theta=0.4$ e $t=10$. A Tabela 6.1 mostra a ordem de convergência para o esquema PFDPUS usando os diferentes pares de parâmetros livres $\alpha$ e $\beta$ com marcha temporal dada por Euler explícito e RK-3. A Tabela 6.2 mostra a ordem de convergência para os esquemas ADBQUICKEST, TOPUS, SDPUS-C1, WACEB e van Albada.

Observa-se por essas tabelas que, no geral, a ordem de convergência alcançada com o esquema PFDPUS, associado ao método de Euler explícito, é superior às ordens atingidas pelos outros esquemas também associados com Euler explícito (ver Tabela 6.2). Em particular, na Tabela 6.1 o esquema PFDPUS atinge nas malhas mais finas (com os parâmetros $\alpha=9$ e $\beta=88$, e, $\alpha=10$ e $\beta=100$ ) segunda ordem de precisão. Quando RK-3 é aplicado no avanço temporal, o esquema PFDPUS apresenta poucas melhoras, mas os esquemas TOPUS, SDPUS-C1, WACEB e van Albada oferecem melhoras bastante significativas. Nota-se que o esquema ADBQUICKEST, interessantemente, tem desempenho muito pobre quando usa-se o método de RK-3. Salienta-se que, no geral, o esquema PFDPUS apresenta ordem convergência superior às ordens apresentadas pelos outros esquemas considerados nesse teste.

A Figura 6.1 ilustra os resultados gerados pelos esquemas PFDPUS $(\alpha=10$ e $\beta=100$ ), ABDQUICKEST, TOPUS, SDPUS-C1, WACEB e van Albada utilizando malha de 320 células computacionais e avanços temporais por Euler explícito e RK-3. A partir dessa figura, pode-se observar que, com exceção do esquema ADBQUICKEST, todos os esquemas geram resultados bastante semelhantes, apresentando uma melhora significativa quando o método de RK-3 é utilizado na marcha no tempo. Com o método de RK-3 empregado no avanço temporal, o esquema ADBQUICKEST mostra-se bastante dissipativo, mas tem resultados em boa concordância com a solução exata quando Euler explícito é aplicado.

\section{Problema 2: (Problema de Zalesak)}

Este problema tem a finalidade de testar a precisão dos esquemas estudados em condições bastante severas de advecção. O problema foi estudado por Zalesak [80] e mais tarde foi usado por Jiang and Shu [30] para verificar o desempenho dos esquemas WENO. A condição inicial contém uma gaussiana, uma onda quadrada, uma onda triangular aguda e uma meia elipse no 


\begin{tabular}{|c|c|c|c|c|c|}
\hline \multirow[t]{2}{*}{$\alpha$ e $\beta$} & \multirow[t]{2}{*}{$N$} & \multicolumn{2}{|c|}{ Euler } & \multicolumn{2}{|c|}{ RK-3 } \\
\hline & & $\mid E_{h} \|_{1}$ & $p$ & $\mid E_{h} \|_{1}$ & $p$ \\
\hline \multirow{6}{*}{0 e -38} & 20 & 0.272189 & - & 0.861933 & - \\
\hline & 40 & 0.107984 & 1.333789 & 0.313535 & 1.458950 \\
\hline & 80 & 0.035864 & 1.590194 & 0.127802 & 1.294709 \\
\hline & 160 & 0.010190 & 1.815372 & 0.039182 & 1.705636 \\
\hline & 320 & 0.002876 & 1.825089 & 0.010266 & 1.932336 \\
\hline & 640 & 0.000808 & 1.830930 & 0.002650 & 1.953984 \\
\hline \multirow[t]{6}{*}{3 e 100} & 20 & 0.168311 & - & 0.501253 & - \\
\hline & 40 & 0.063522 & 1.405791 & 0.173533 & 1.530327 \\
\hline & 80 & 0.018754 & 1.760087 & 0.054323 & 1.675582 \\
\hline & 160 & 0.005256 & 1.835231 & 0.014703 & 1.885484 \\
\hline & 320 & 0.001452 & 1.855553 & 0.003902 & 1.913723 \\
\hline & 640 & 0.000421 & 1.785370 & 0.001071 & 1.864885 \\
\hline \multirow[t]{6}{*}{9 e 88} & 20 & 0.110077 & - & 0.266199 & - \\
\hline & 40 & 0.038791 & 1.504712 & 0.067533 & 1.978835 \\
\hline & 80 & 0.010347 & 1.906549 & 0.024373 & 1.470320 \\
\hline & 160 & 0.002544 & 2.023774 & 0.006365 & 1.936941 \\
\hline & 320 & 0.000610 & 2.060291 & 0.001622 & 1.972106 \\
\hline & 640 & 0.000147 & 2.049669 & 0.000411 & 1.981452 \\
\hline \multirow[t]{6}{*}{10 e 100} & 20 & 0.098432 & - & 0.237489 & - \\
\hline & 40 & 0.036614 & 1.426723 & 0.058259 & 2.027295 \\
\hline & 80 & 0.009889 & 1.888497 & 0.024028 & 1.277803 \\
\hline & 160 & 0.002426 & 2.027099 & 0.006431 & 1.901595 \\
\hline & 320 & 0.000583 & 2.056766 & 0.001647 & 1.965189 \\
\hline & 640 & 0.000141 & 2.052616 & 0.000416 & 1.984726 \\
\hline
\end{tabular}

Tabela 6.1: Teste de convergência para o problema da condição suave usando-se o esquema PFDPUS com os parâmetros $\alpha$ e $\beta$ em $t=10$. 


\begin{tabular}{|c|c|c|c|c|c|}
\hline \multirow[t]{2}{*}{ Esquemas } & \multirow[t]{2}{*}{$N$} & \multicolumn{2}{|c|}{ Euler } & \multicolumn{2}{|c|}{ RK-3 } \\
\hline & & $\mid E_{h} \|_{1}$ & $p$ & $\mid E_{h} \|_{1}$ & $p$ \\
\hline \multirow[t]{6}{*}{ ADBQUICKEST } & 20 & 0.244765 & - & 1.047407 & - \\
\hline & 40 & 0.098444 & 1.314020 & 0.786763 & 0.412820 \\
\hline & 80 & 0.044492 & 1.145762 & 0.511646 & 0.620784 \\
\hline & 160 & 0.021248 & 1.066242 & 0.300716 & 0.766745 \\
\hline & 320 & 0.010380 & 1.033538 & 0.166049 & 0.856792 \\
\hline & 640 & 0.005127 & 1.017461 & 0.088286 & 0.911356 \\
\hline \multirow[t]{6}{*}{ TOPUS } & 20 & 0.342753 & - & 0.534726 & - \\
\hline & 40 & 0.469017 & -0.452469 & 0.184147 & 1.537943 \\
\hline & 80 & 0.238222 & 0.977333 & 0.065636 & 1.488298 \\
\hline & 160 & 0.111950 & 1.089446 & 0.026353 & 1.316511 \\
\hline & 320 & 0.058087 & 0.946566 & 0.011507 & 1.195402 \\
\hline & 640 & 0.029976 & 0.954408 & 0.005361 & 1.102027 \\
\hline \multirow[t]{6}{*}{ SDPUS-C1 } & 20 & 0.406838 & - & 0.393993 & - \\
\hline & 40 & 0.532575 & -0.388528 & 0.137525 & 1.518479 \\
\hline & 800 & 0.288005 & 0.886892 & 0.050453 & 1.446680 \\
\hline & 160 & 0.119386 & 1.270457 & 0.022241 & 1.181745 \\
\hline & 320 & 0.056778 & 1.072229 & 0.010491 & 1.084004 \\
\hline & 640 & 0.030001 & 0.920337 & 0.005110 & 1.037842 \\
\hline \multirow[t]{6}{*}{ WACEB } & 20 & 0.492638 & - & 0.280319 & - \\
\hline & 40 & 0.363976 & 0.436687 & 0.091247 & 1.619228 \\
\hline & 80 & 0.176467 & 1.044442 & 0.026406 & 1.788904 \\
\hline & 160 & 0.084492 & 1.062514 & 0.007276 & 1.859613 \\
\hline & 320 & 0.052460 & 0.687605 & 0.001914 & 1.926448 \\
\hline & 640 & 0.027995 & 0.906033 & 0.000493 & 1.956194 \\
\hline \multirow[t]{6}{*}{ van Albada } & 20 & 0.355149 & - & 0.600431 & - \\
\hline & 40 & 0.508640 & -0.518219 & 0.230468 & 1.381436 \\
\hline & 80 & 0.329642 & 0.625743 & 0.096817 & 1.251236 \\
\hline & 160 & 0.140964 & 1.225579 & 0.038734 & 1.321657 \\
\hline & 320 & 0.069004 & 1.030570 & 0.015729 & 1.300137 \\
\hline & 640 & 0.040179 & 0.780253 & 0.006845 & 1.200360 \\
\hline
\end{tabular}

Tabela 6.2: Teste de convergência para o problema da condição suave usando-se os esquemas ABDQUICKEST, TOPUS, SDPUS-C1, WACEB e van Albada em $t=10$. 


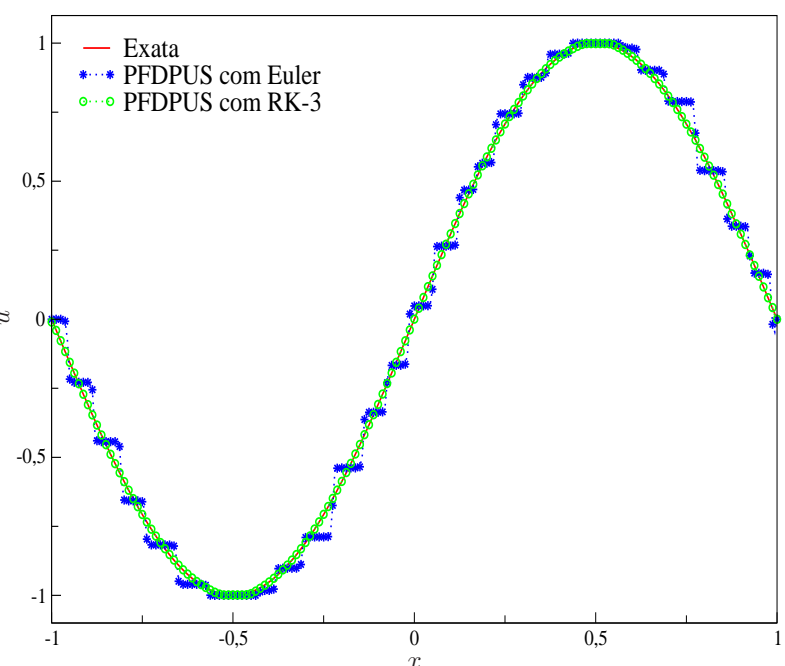

(a)

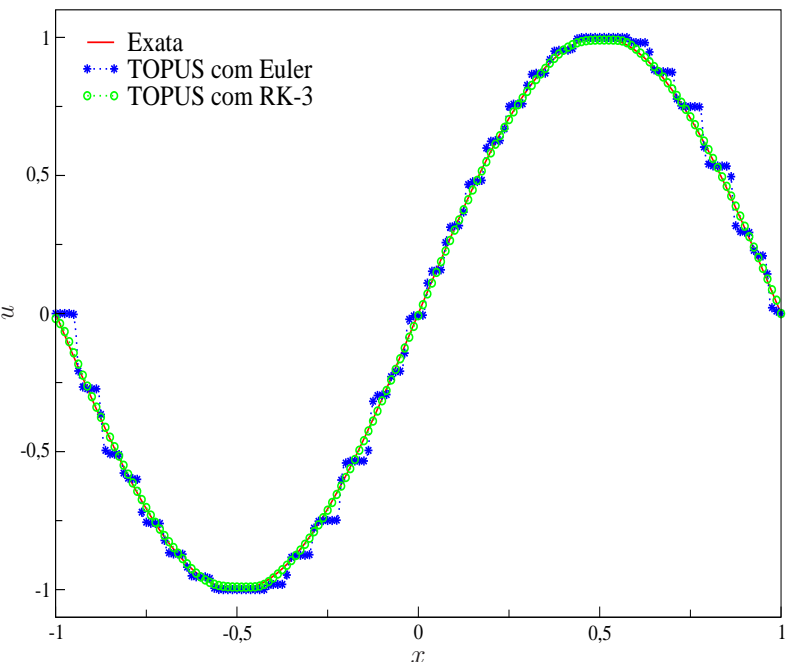

(c)

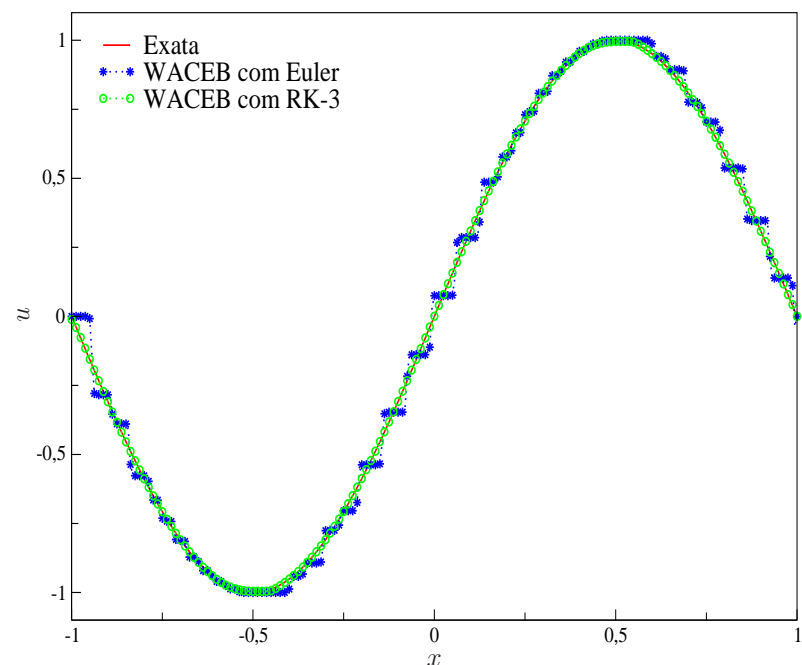

(e)

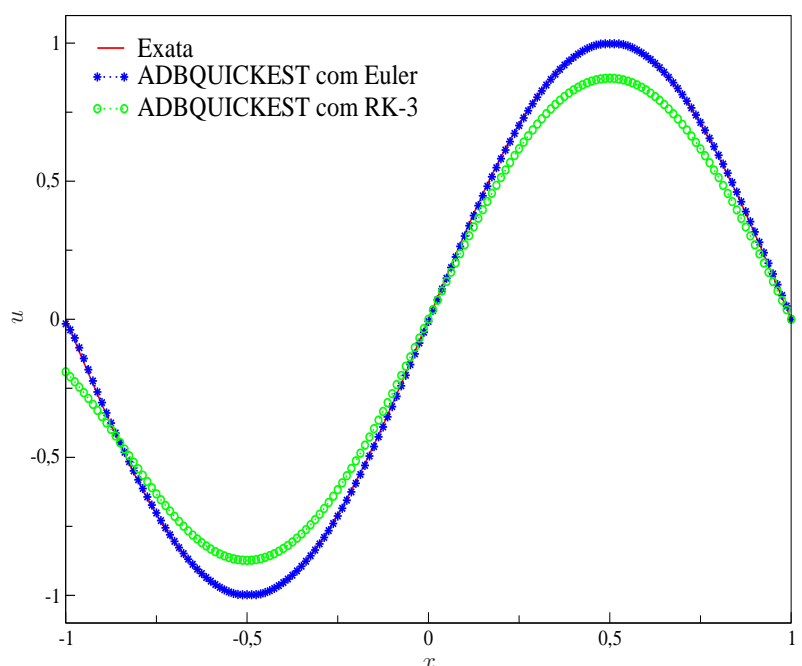

(b)

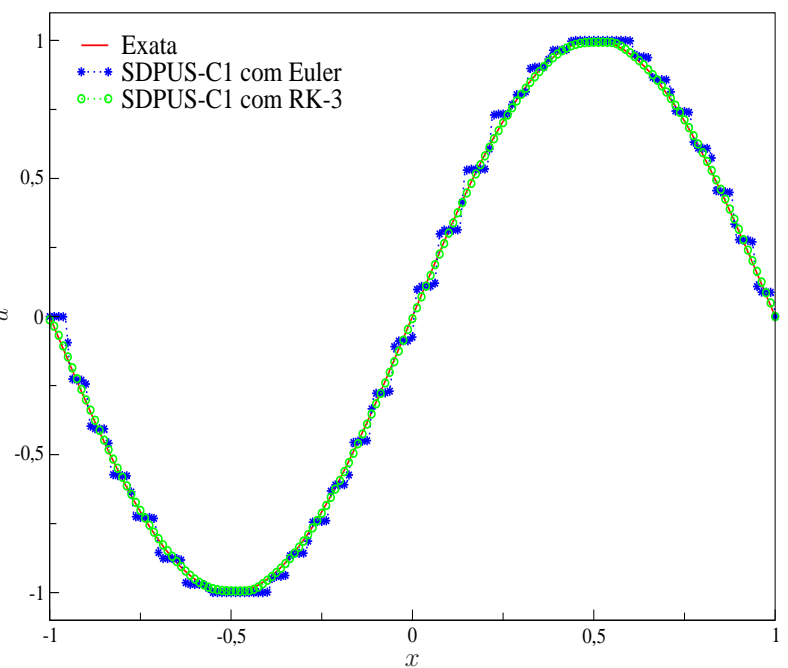

(d)

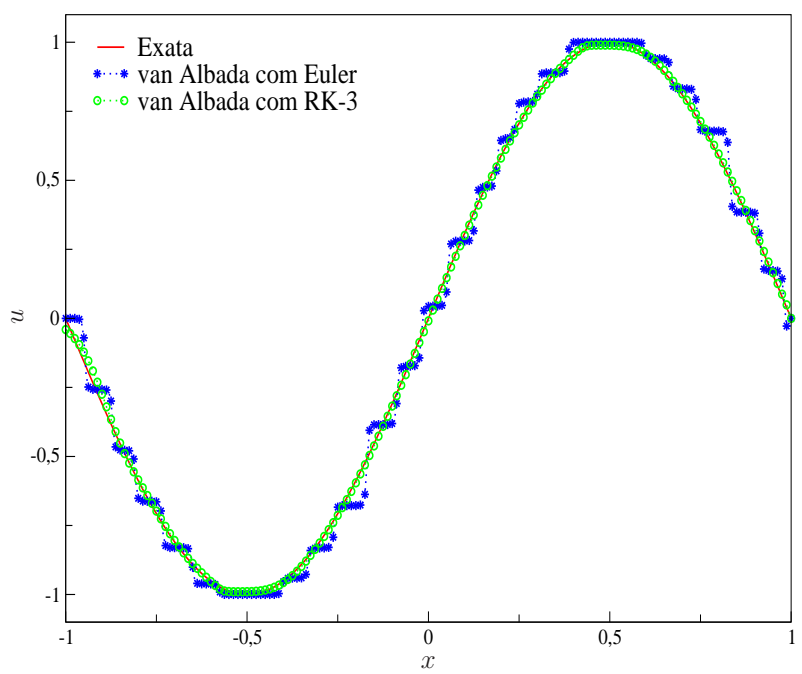

(f)

Figura 6.1: Comparação dos resultados numéricos obtidos para o problema da condição suave com os métodos de Euler explícito e RK-3 em $t=10$, usando 320 células computacionais e $\theta=0.4$. 
intervalo $[-1,1]$. Em resumo, a condição inicial é

$$
u(x, 0)=\left\{\begin{array}{lrc}
\frac{1}{6}\left(G\left(x, \beta_{1}, z-\delta\right)+G\left(x, \beta_{1}, z+\delta_{1}\right)+4 G\left(x, \beta_{1}, z\right)\right), & \text { se } & -0.8 \leq x \leq-0.6, \\
1, & \text { se } & -0.4 \leq x \leq-0.2, \\
1-|10(x-0.1)|, & \text { se } & 0 \leq x \leq 0.2, \\
\frac{1}{6}\left(F\left(x, \alpha_{1}, c-\delta_{1}\right)+F\left(x, \alpha_{1}, c+\delta_{1}\right)+4 F\left(x, \alpha_{1}, c\right)\right), & \text { se } & 0.4 \leq x \leq 0.6, \\
0, & \text { se } & x \notin[-0.8,0.6],
\end{array}\right.
$$

onde

$$
G\left(x, \beta_{1}, z\right)=e^{-\beta_{1}(x-z)^{2}} \quad \text { e } \quad F\left(x, \alpha_{1}, c\right)=\sqrt{\max \left(1-\alpha_{1}^{2}(x-c)^{2}, 0\right)} .
$$

As constantes usadas são $c=0.5, z=-0.7, \delta_{1}=0.005, \alpha_{1}=10$ e $\beta_{1}=\frac{\log 2}{36 \delta_{1}^{2}}$. Esse problema contém várias estruturas que são difíceis de transportar com fidelidade, o que permite testar várias características importantes dos esquemas estudados, tais como a precisão em regiões suaves, a resolução próxima às descontinuidades e a forma com que os picos e vales são capturados. As condições de contorno aplicadas são periódicas. Para simulação, malhas 200, 400, 800 e 1600 células computacionais, $\theta=0.4$ e $t=1$ são adotados.

A Tabela 6.3 mostra os erros na norma $L_{1}$ e a ordem de convergência para o esquema PFDPUS com os diferentes pares de parâmetros livres $\alpha$ e $\beta$. A Tabela 6.4 é similar a Tabela 6.3, mas com o uso dos esquemas ADBQUICKEST, TOPUS, SDPUS-C1, WACEB e van Albada. Pode-se observar nessas tabelas, que quando o avanço temporal dá-se pela aplicação do método de Euler explícito podem aparecer ordens de convergência negativas, como aconteceu com os esquemas PFDPUS (com parâmetros $\alpha=0$ e $\beta=-38$ ) e van Albada na malha 400. As tabelas também mostram que ao empregar o método de RK-3, em geral, as estimativas para a ordem de convergência melhoram, com exceção do esquema ADBQUICKEST.

No caso particular do esquema PFDPUS, vê-se a partir da Tabela 6.3 que a combinação $\alpha=10$ e $\beta=100$, associada com Euler explícito, apresenta uma melhor estimativa para a ordem de convergência. No entanto, a combinação $\alpha=0$ e $\beta=-38$ quando associada ao RK-3 fornece os melhores resultados. Vale ressaltar que para condições descontínuas como as quais são consideradas nesse problema, a ordem de convergência esperada é 1, uma vez que em regiões com altos gradientes os esquemas considerados aplicam baixa ordem.

A Figura 6.2 apresenta os resultados numéricos obtidos com o esquema PFDPUS (para os diferentes pares de parâmetros livres $\alpha$ e $\beta$ ) quando o avanço temporal é dado por Euler explícito. Pode-se notar por esta figura que o esquema PFDPUS com o par de parâmetros $\alpha=10$ e $\beta=100$ fornece a melhor aproximação, ao passo que o esquema com $\alpha=0$ e $\beta=-38$ gera uma solução bastante dissipativa (ver Ampliação 1 e 2). No entanto, com qualquer uma das combinações dos parâmetros, o esquema PFDPUS apresenta bons resultados na captura da onda quadrada (ver Ampliação 1), na solução dos vales e regiões suaves. Além disso, nota-se que todos os resultados apresentam o fenômeno squaring effect nos picos da gaussiana, da onda triangular aguda e na meia elipse. Em síntese, para esse problema, o esquema PFDPUS com parâmetros $\alpha=10$ e $\beta=100$ apresenta os melhores resultados. 


\begin{tabular}{|c|c|c|c|c|c|}
\hline \multirow[t]{2}{*}{$\alpha \mathrm{e} \beta$} & \multirow[t]{2}{*}{$N$} & \multicolumn{2}{|c|}{ Euler } & \multicolumn{2}{|c|}{ RK-3 } \\
\hline & & $\mid E_{h} \|_{1}$ & $p$ & $\mid E_{h} \|_{1}$ & $p$ \\
\hline \multirow[t]{4}{*}{0 e -38} & 200 & 0.080317 & - & 0.234321 & - \\
\hline & 400 & 0.094017 & -0.227215 & 0.106048 & 1.143767 \\
\hline & 800 & 0.071172 & 0.401607 & 0.054034 & 0.972769 \\
\hline & 1600 & 0.046465 & 0.615189 & 0.026533 & 1.026072 \\
\hline \multirow[t]{4}{*}{3 e 100} & 200 & 0.099124 & - & 0.126977 & - \\
\hline & 400 & 0.078527 & 0.336043 & 0.062701 & 1.017992 \\
\hline & 800 & 0.043580 & 0.849538 & 0.030097 & 1.058855 \\
\hline & 1600 & 0.019567 & 1.155238 & 0.015241 & 0.981729 \\
\hline \multirow[t]{4}{*}{9 e 88} & 200 & 0.107022 & - & 0.078764 & - \\
\hline & 400 & 0.083693 & 0.354736 & 0.042309 & 0.896550 \\
\hline & 800 & 0.041713 & 1.004599 & 0.019795 & 1.095836 \\
\hline & 1600 & 0.019661 & 1.085199 & 0.010091 & 0.972042 \\
\hline \multirow[t]{4}{*}{10 e 100} & 200 & 0.109219 & - & 0.074044 & - \\
\hline & 400 & 0.080712 & 0.436368 & 0.040394 & 0.874254 \\
\hline & 800 & 0.038480 & 1.068674 & 0.019073 & 1.082595 \\
\hline & 1600 & 0.019679 & 0.967433 & 0.009680 & 0.978514 \\
\hline
\end{tabular}

Tabela 6.3: Teste de convergência para o problema de Zalesak usando-se o esquema PFDPUS com os parâmetros $\alpha$ e $\beta$ em $t=1$.

A Figura 6.3 mostra os resultados numéricos obtidos com os esquemas PFDPUS $(\alpha=10$ e $\beta=100$ ), ADBQUICKSET, TOPUS, SDPUS-C1, WACEB e van Albada. Nessas simulações a marcha no tempo é feita pelo método de Euler explícito. Observa-se que o esquema ADBQUICKEST é bastante dissipativo e então suaviza as regiões de altos gradientes. Os demais esquemas têm comportamentos bem semelhantes, mas vale ressaltar que os esquemas PFDPUS e WACEB apresentam os melhores resultados na captura da onda quadrada. 


\begin{tabular}{lcccccc}
\hline Esquema & $N$ & \multicolumn{2}{c}{ Euler } & & \multicolumn{2}{c}{ RK-3 } \\
\cline { 3 - 4 } \cline { 6 - 7 } & & $\left\|E_{h}\right\|_{1}$ & $p$ & & $\left\|E_{h}\right\|_{1}$ & $p$ \\
\hline ADBQUICKEST & 200 & 0.077340 & - & & 0.409942 & - \\
& 400 & 0.039963 & 0.952569 & & 0.287658 & 0.511064 \\
TOPUS & 800 & 0.021217 & 0.913423 & & 0.191205 & 0.589234 \\
& 1600 & 0.011613 & 0.869526 & & 0.122456 & 0.642858 \\
& 200 & 0.092642 & - & & 0.140457 & - \\
& 400 & 0.069985 & 0.404631 & & 0.069592 & 1.013145 \\
SDPUS-C1 & 800 & 0.035873 & 0.964122 & & 0.035740 & 0.961376 \\
& 1600 & 0.018526 & 0.953347 & & 0.019089 & 0.904765 \\
& 200 & 0.100919 & - & & 0.110713 & - \\
WACEB & 400 & 0.071497 & 0.497253 & & 0.058173 & 0.928409 \\
& 800 & 0.035170 & 1.023544 & & 0.029986 & 0.956059 \\
& 1600 & 0.019214 & 0.872206 & & 0.016479 & 0.863700 \\
& 200 & 0.111046 & - & & 0.091451 & - \\
& 400 & 0.063497 & 0.806381 & & 0.049265 & 0.892436 \\
van Albada & 800 & 0.026035 & 1.286243 & & 0.025423 & 0.954455 \\
& 1600 & 0.015329 & 0.764182 & & 0.013815 & 0.879922 \\
& 200 & 0.095356 & - & & 0.161156 & - \\
& 400 & 0.110007 & -0.206201 & 0.077684 & 1.052773 \\
& 800 & 0.046295 & 1.248652 & 0.040556 & 0.937690 \\
& 1600 & 0.025602 & 0.854632 & 0.021951 & 0.885618 \\
\hline
\end{tabular}

Tabela 6.4: Teste de convergência para o problema de Zalesak usando-se os esquemas ADBQUICKEST, TOPUS, SDPUS-C1, WACEB e van Albada em $t=1$. 


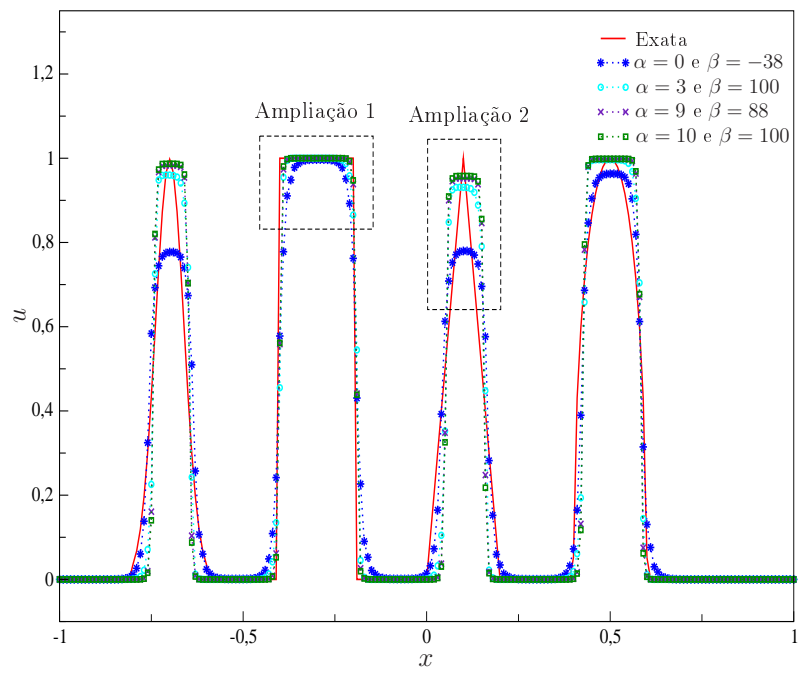

(a) PFDPUS com os parâmetros $\alpha$ e $\beta$

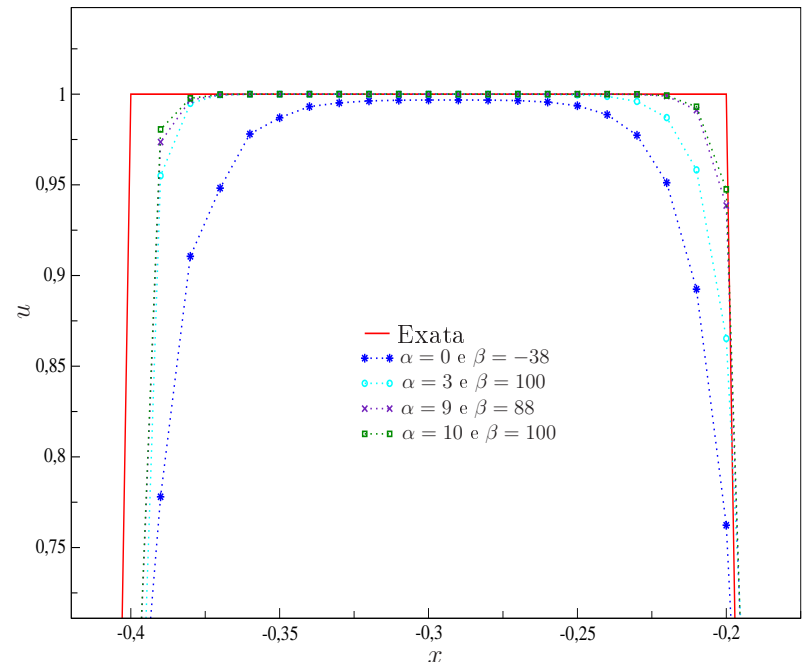

(b) Ampliação 1

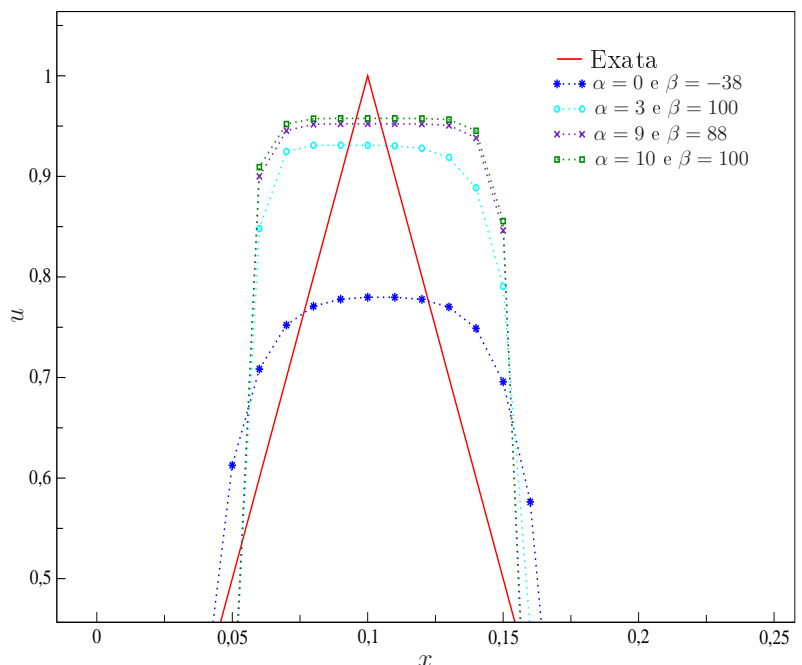

(c) Ampliação 2

Figura 6.2: Resultados numéricos obtidos para o problema de Zalesak em $t=1$ com o esquema PFDPUS, usando 200 células computacionais e $\theta=0.4$. 


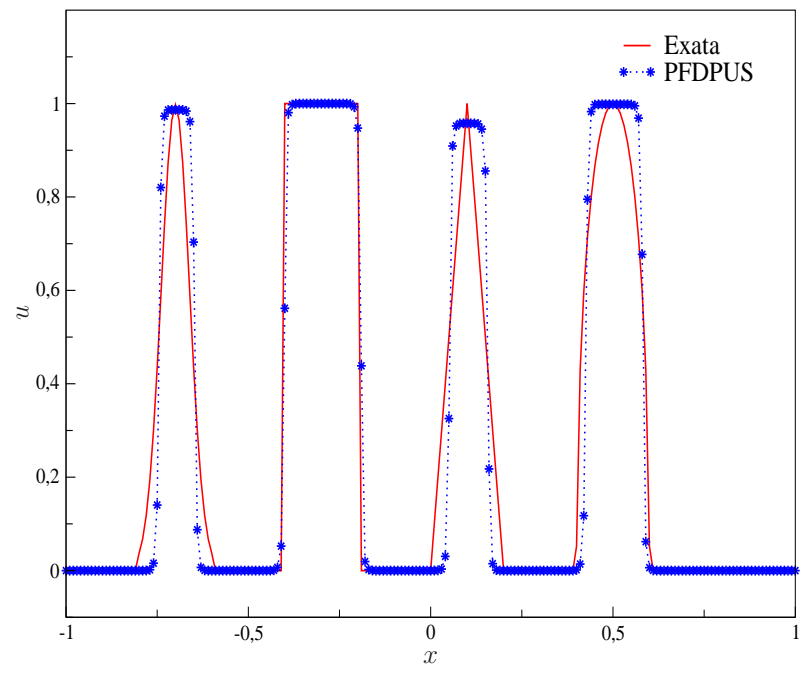

(a)

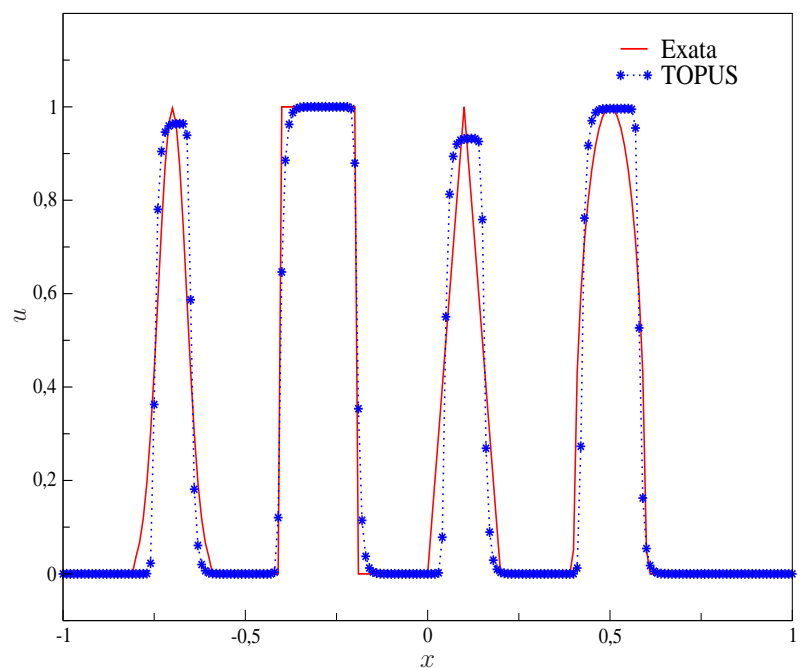

(c)

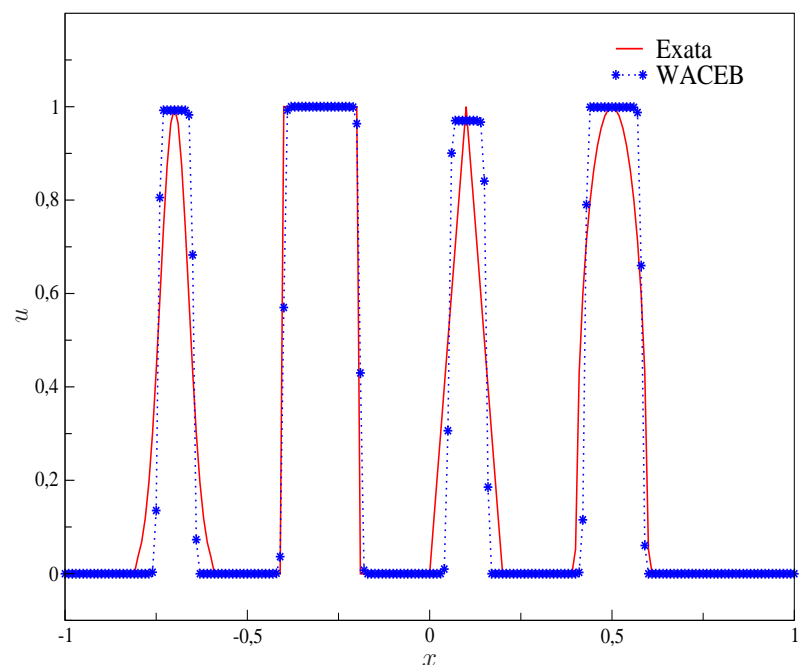

(e)

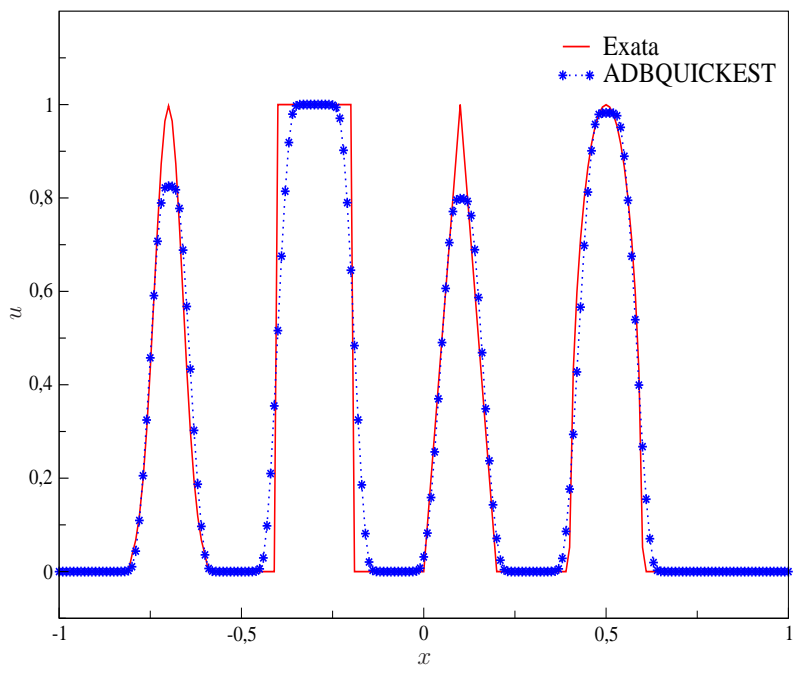

(b)

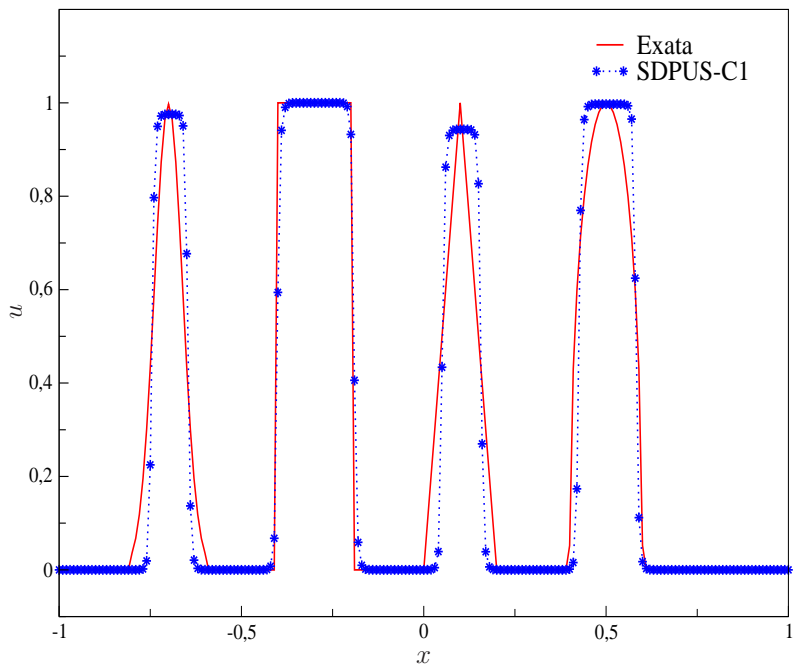

(d)

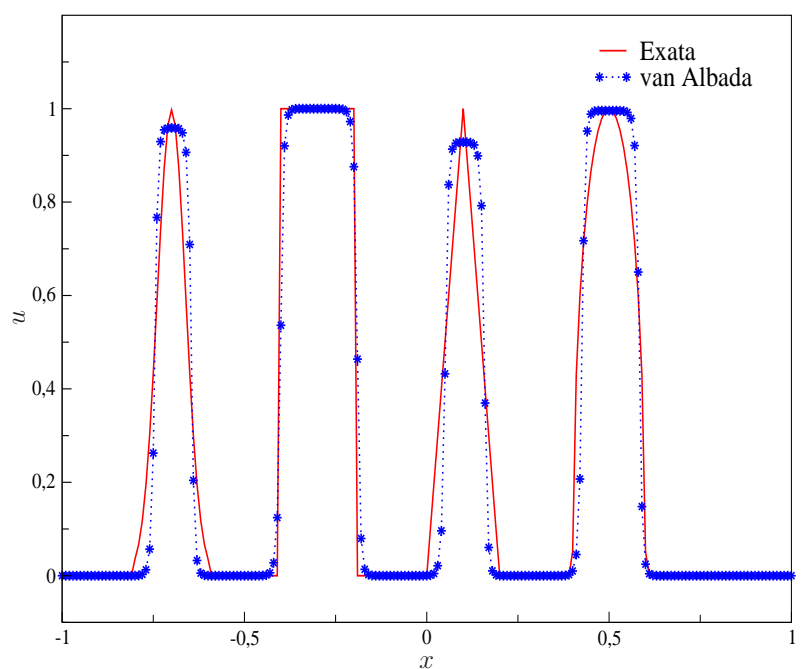

(f)

Figura 6.3: Resultados numéricos obtidos para o problema de Zalesak em $t=1$, usando 200 células computacionais e $\theta=0.4$. 


\section{Problema 3: (Onda quadrada)}

Este teste analisa o desempenho dos esquemas estudados na captura de descontinuidades. A condição inicial consiste em uma onda quadrada no domínio $[-1,1]$ dada por

$$
u(x, 0)= \begin{cases}1, & \text { se } \quad-\frac{1}{5} \leq x \leq \frac{1}{5}, \\ 0, & \text { se } \quad x \notin\left[-\frac{1}{5}, \frac{1}{5}\right] .\end{cases}
$$

As condições de contorno aplicadas são periódicas e o avanço temporal dá-se pela aplicação do método de Euler explícito. Os resultados numéricos obtidos numa malha de 320 células computacionais, $\theta=0.6$ e $t=2.8$ são mostrados na Figura 6.4, onde pode-se observar que os resultados obtidos com os esquemas TOPUS, WACEB e SDPUS-C1 apresentam severas oscilações à esquerda das descontinuidades. Em contraste, os esquemas PFDPUS $(\alpha=10$ e $\beta=100$ ), ADBQUICKEST e van Albada resolvem bem a onda quadrada, sem oscilações espúrias e excessiva dissipação. Em particular, os resultados com PFDPUS competem bem com os resultados de van Albada.

Neste problema, também é feita a análise da restrição TVD para o esquema PFDPUS com o par de parâmetros $\alpha=10$ e $\beta=100$. Para tanto, calcula-se a TV, dada por (3.6), a cada passo de tempo, com 100, 200 e 400 células computacionais, $\theta=0.6$ e $t=4$. Os resultados são plotados no plano $T V(u(t)) \perp t$. Para que o esquema PFDPUS satisfaça as restrições TVD, este deve apresentar a TV constante, constante por partes ou decrescente. Também é importante constatar se a TV numérica converge para a TV exata, que é dada pela função $T V(u(t))=2$. Os resultados são ilustrados na Figura 6.5. Observa-se que as aproximações são satisfatórias e que o esquema PFDPUS com parâmetros $\alpha=10$ e $\beta=100$ satisfaz as restrições TVD, uma vez que as TVs numéricas calculadas apresentam um comportamento decrescente ou constante. Além disso, nota-se claramente que, com o refinamento da malha, as TVs numéricas obtidas convergem para a TV exata.

\subsection{Equação de Burgers - sem viscosidade}

Nesta seção, resolve-se a equação de Burgers sem viscosidade que é formulada pela lei de conservação 1D (2.1), admitindo variável conservada (2.8) e fluxo numérico (2.9). Para as simulações, três condições iniciais são adotadas. As soluções numéricas geradas pelo esquema PFDPUS com diferentes pares de parâmetros livres $(\alpha=0$ e $\beta=-38, \alpha=3$ e $\beta=100, \alpha=9$ e $\beta=88$, e, $\alpha=10$ e $\beta=100$ ) são confrontadas com soluções exatas e soluções numéricas geradas pelos esquemas ADBQUICKEST, TOPUS, SDPUS-C1, WACEB, van Albada e Superbee. Também analisa-se a restrição TVD e calcula-se estimativas para ordem de convergência. Para a marcha no tempo, ambos os métodos de Euler explícito (ver (5.1)) e RK-3 (ver (5.2)-(5.4)) são utilizados. 


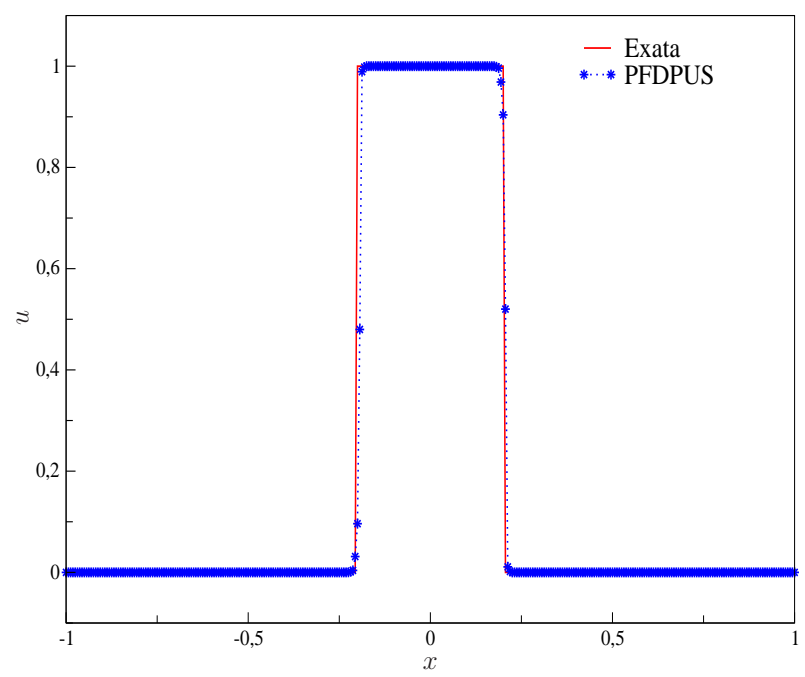

(a)

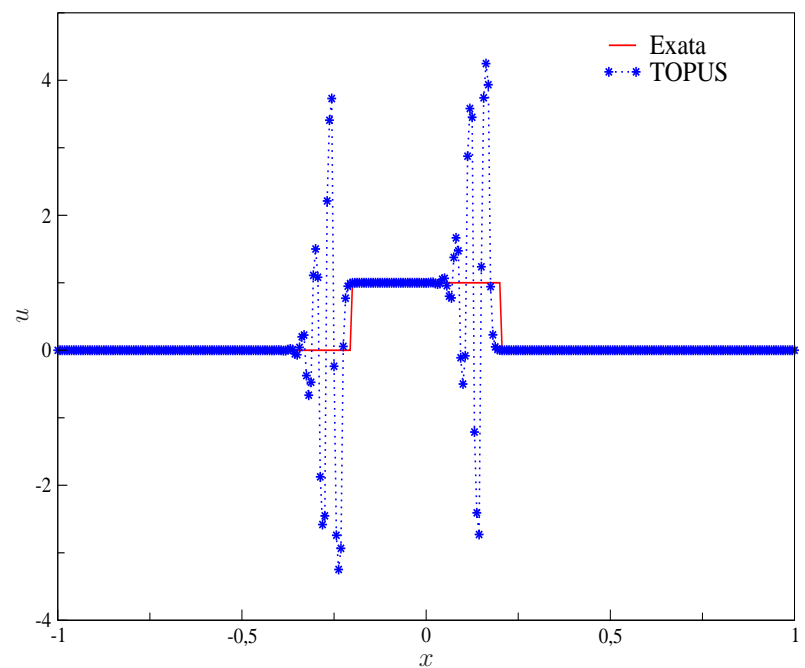

(c)

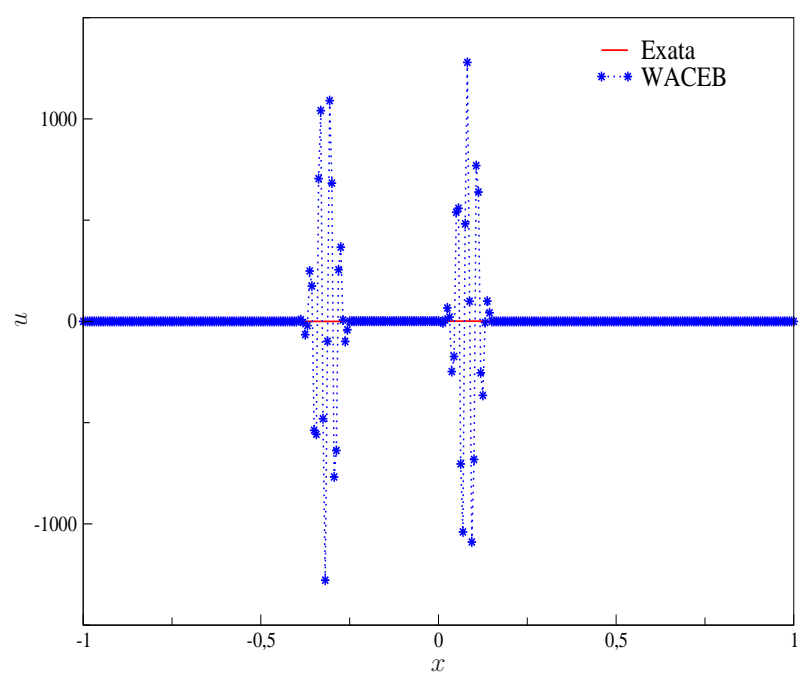

(e)

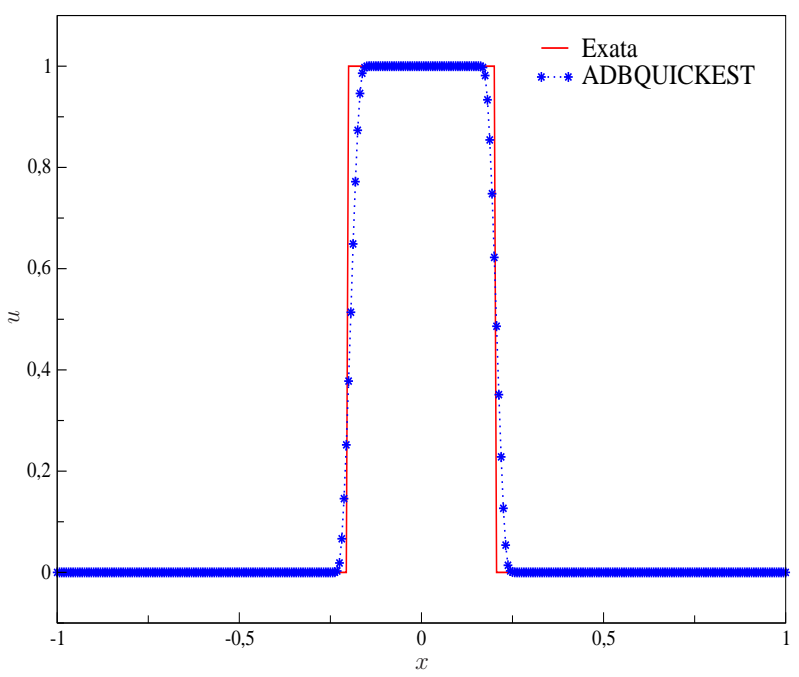

(b)

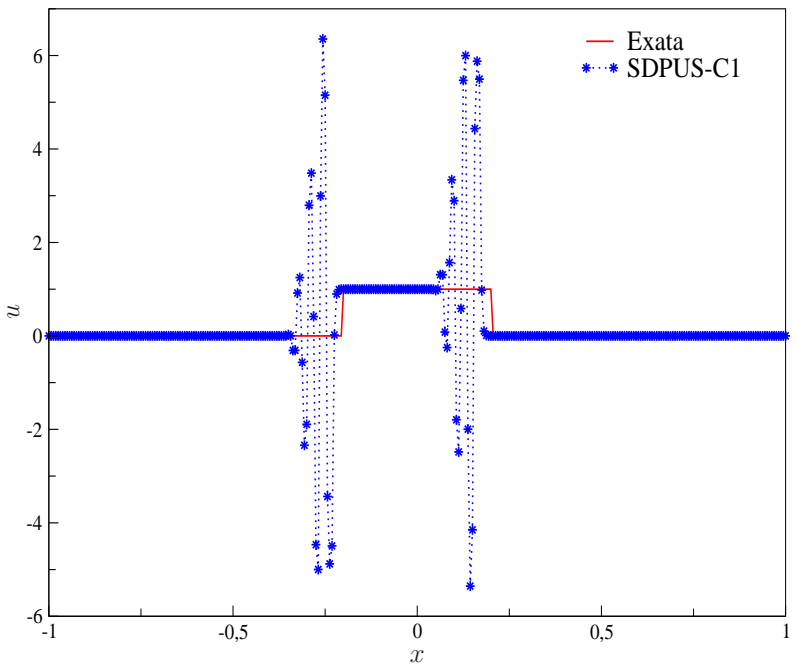

(d)

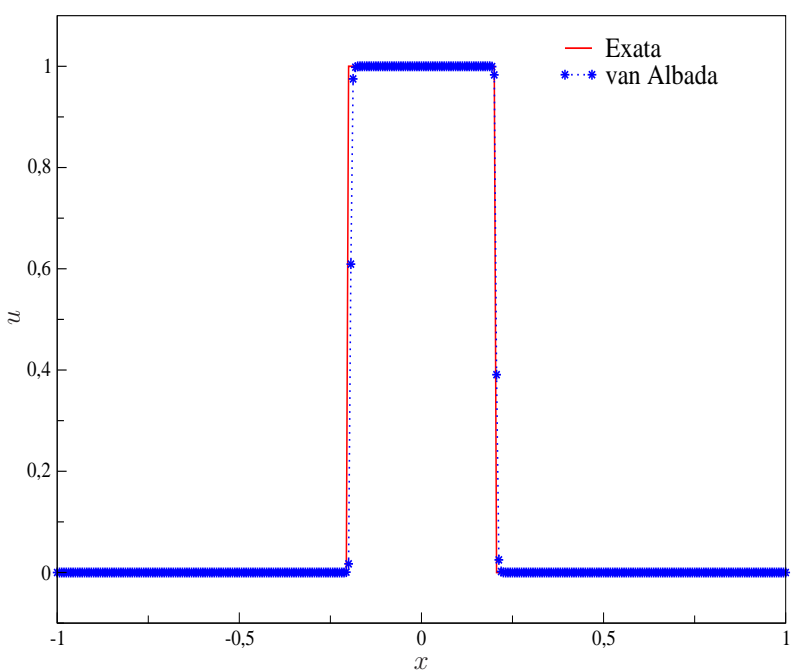

(f)

Figura 6.4: Resultados numéricos obtidos para o problema da onda quadrada em $t=2.8$, usando 320 células computacionais e $\theta=0.6$. 


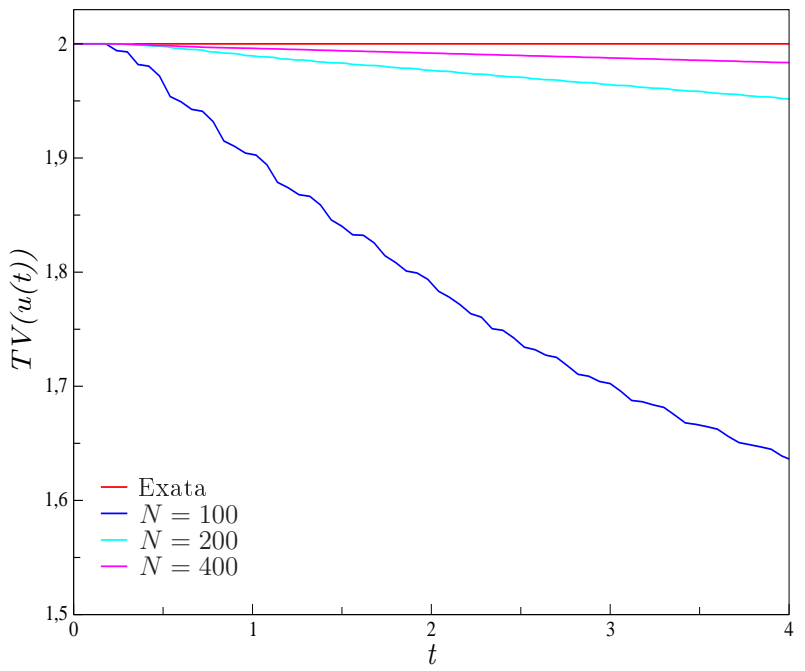

Figura 6.5: Análise da restrição TVD para o problema da onda quadrada usando o esquema PFDPUS em $t=4$.

\section{Problema 4: (Condição inicial suave)}

Neste problema considera-se a equação de Burgers suplementada com condição inicial suave definida em $[-1,1]$ da forma

$$
u(x, 0)=1+\frac{1}{2} \operatorname{sen}(\pi x)
$$

Aqui $t=0.32$ é escolhido para calcular a ordem de convergência e $t=1.52$ para verificar a captura do choque. No cálculo das soluções numéricas, são usadas condições de contorno periódicas. Neste problema, como a solução exata não é conhecida, as estimativas para ordem de convergência na norma $L_{1}$ são computadas com base em três malhas (ver (2.70)). Para tanto, malhas de 20, 40, 80, 160, 320 e 640 células computacionais e $\theta=0.4$ são considerados.

Nas Tabelas 6.5 e 6.6 encontram-se os erros e as ordens de convergência obtidas pelos esquemas PFDPUS (com os diferentes pares de parâmetros livres $\alpha$ e $\beta$ ), ADBQUICKEST, SDPUS-C1 e van Albada. Pode-se observar em ambas as tabelas que, quando o avanço temporal dá-se pelo método de RK-3, todos os esquemas apresentam melhoras na ordem de convergência. Além disso, com esse mesmo método de marcha no tempo o esquema PFDPUS (com os quatro pares de parâmetros livres) atinge segunda ordem de precisão em todas as malhas consideradas, mostrando ordem superior às geradas pelos demais esquemas.

A Figura 6.6 mostra as comparações entre a solução exata de Zhen-huan Teng [67] para esse problema e as soluções numéricas obtidas com os esquemas PFDPUS ( $\alpha=0$ e $\beta=-38$ ), ADBQUICKEST, SDPUS-C1 e van Albada em $t=1.52$. Nas simulações são usados ambos os métodos Euler explícito e RK-3 para o avanço temporal, e 320 células computacionais. Observa-se que, ao se usar no avanço temporal o método de Euler explícito, todos os esquemas geram oscilações próximas ao choque, com o esquema SDPUS-C1 fornecendo o pior resultado. Por outro lado, ao se usar o RK-3, todas as soluções numéricas estão em boa concordância com a solução exata de Zhen-huan Teng considerada. 


\begin{tabular}{|c|c|c|c|c|c|}
\hline \multirow[t]{2}{*}{$\alpha$ e $\beta$} & \multirow[t]{2}{*}{$N$} & \multicolumn{2}{|c|}{ Euler } & \multicolumn{2}{|c|}{ RK-3 } \\
\hline & & $\mid E_{h} \|_{1}$ & $p$ & $E_{h} \|_{1}$ & $p$ \\
\hline \multirow[t]{4}{*}{0 e -38} & 40 & 0.010589 & 1.844457 & 0.008902 & 2.079148 \\
\hline & 80 & 0.005926 & 1.842440 & 0.003865 & 2.037533 \\
\hline & 160 & 0.003313 & 1.841277 & 0.001751 & 2.112922 \\
\hline & 320 & 0.001677 & 1.980483 & 0.000829 & 2.061340 \\
\hline \multirow[t]{4}{*}{3 e 100} & 40 & 0.014215 & 1.486933 & 0.007861 & 2.140855 \\
\hline & 80 & 0.009634 & 1.402558 & 0.003891 & 2.119497 \\
\hline & 160 & 0.005363 & 1.590231 & 0.001683 & 2.133333 \\
\hline & 320 & 0.002653 & 1.914255 & 0.000809 & 2.070039 \\
\hline \multirow[t]{4}{*}{9 e 88} & 40 & 0.013040 & 1.613282 & 0.007560 & 2.153378 \\
\hline & 80 & 0.009106 & 1.621053 & 0.003743 & 2.131662 \\
\hline & 160 & 0.004462 & 1.939367 & 0.001665 & 2.119349 \\
\hline & 320 & 0.002989 & 1.691429 & 0.000802 & 2.063370 \\
\hline \multirow[t]{4}{*}{10 e 100} & 40 & 0.013631 & 1.655543 & 0.008056 & 2.108513 \\
\hline & 80 & 0.009442 & 1.626706 & 0.003808 & 2.165263 \\
\hline & 160 & 0.005145 & 1.837564 & 0.001679 & 2.134228 \\
\hline & 320 & 0.003088 & 1.746930 & 0.000807 & 2.068174 \\
\hline
\end{tabular}

Tabela 6.5: Teste de convergência para o problema da condição suave usando-se o esquema PFDPUS com os parâmetros $\alpha$ e $\beta$ em $t=0.32$.

\begin{tabular}{lcccccc}
\hline Esquemas & $N$ & \multicolumn{2}{c}{ Euler } & & \multicolumn{2}{c}{ RK-3 } \\
\cline { 3 - 4 } \cline { 6 - 7 } & & $\left\|E_{h}\right\|_{1}$ & $p$ & & $\left\|E_{h}\right\|_{1}$ & $p$ \\
\hline ADBQUICKEST & 40 & 0.004223 & 1.602871 & & 0.007628 & 2.012328 \\
& 80 & 0.002504 & 1.766410 & & 0.003746 & 1.932096 \\
& 160 & 0.001389 & 1.867078 & & 0.001866 & 1.965324 \\
SDPUS-C1 & 320 & 0.000723 & 1.926219 & & 0.000936 & 1.978984 \\
& 40 & 0.014503 & 1.133535 & & 0.006579 & 1.989104 \\
& 80 & 0.006230 & 2.199303 & & 0.003329 & 2.036667 \\
& 160 & 0.006059 & 1.451869 & & 0.001615 & 2.031190 \\
van Albada & 320 & 0.002864 & 1.893271 & & 0.000790 & 2.024446 \\
& 40 & 0.011278 & 1.350497 & & 0.007020 & 1.956263 \\
& 80 & 0.006377 & 1.798802 & & 0.003652 & 1.983515 \\
& 160 & 0.003694 & 1.800378 & & 0.001695 & 2.049593 \\
& 320 & 0.002050 & 1.782804 & & 0.000824 & 2.043367 \\
\hline
\end{tabular}

Tabela 6.6: Teste de convergência para o problema da condição suave usando-se os esquemas ABDQUICKEST, SDPUS-C1 e van Albada em $t=0.32$. 


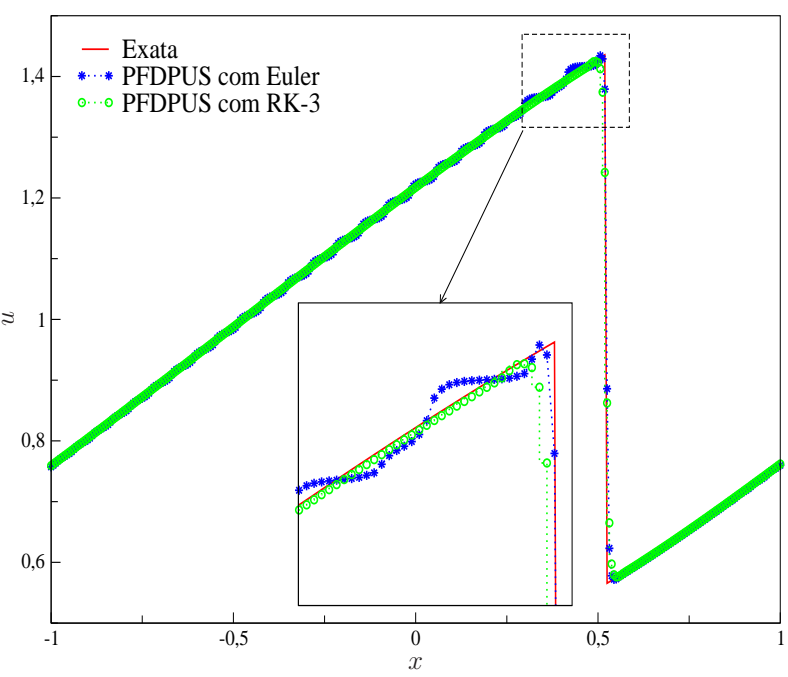

(a)

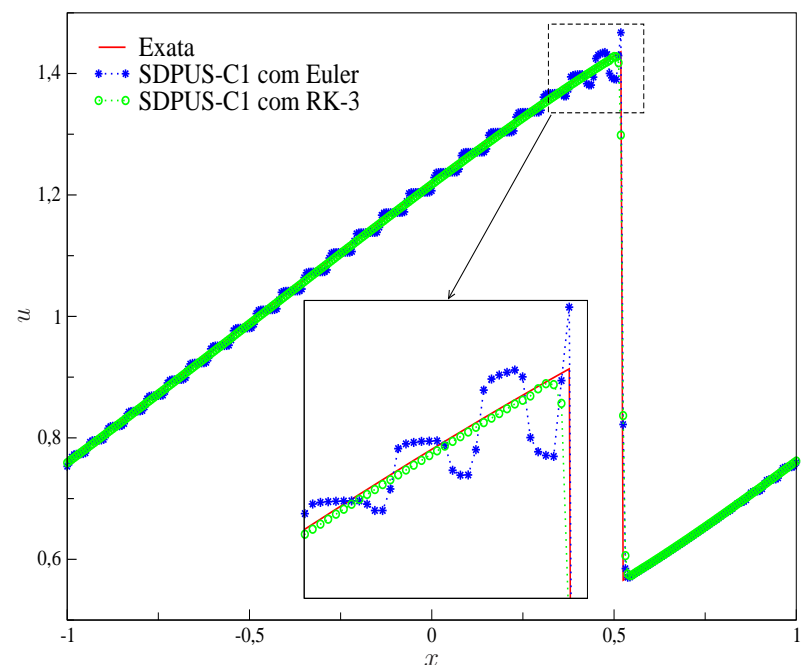

(c)

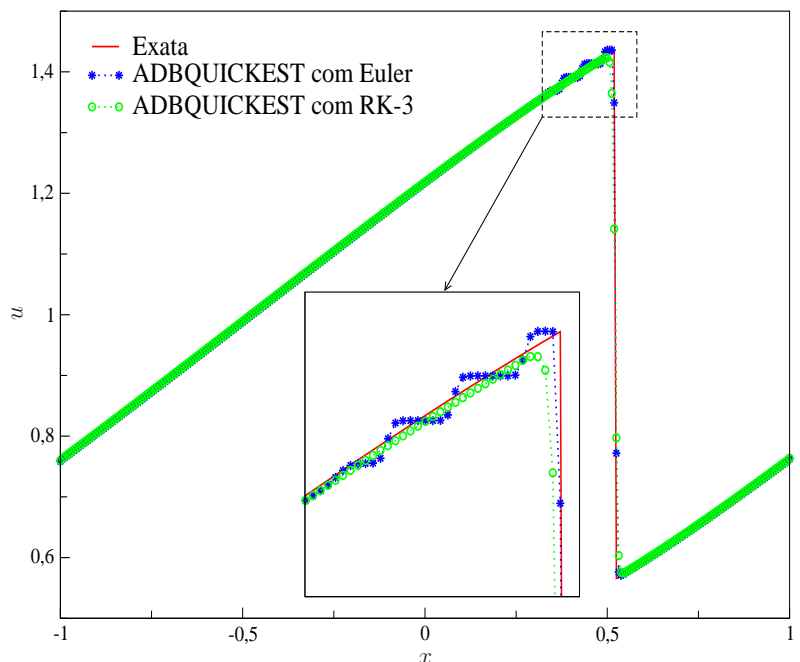

(b)

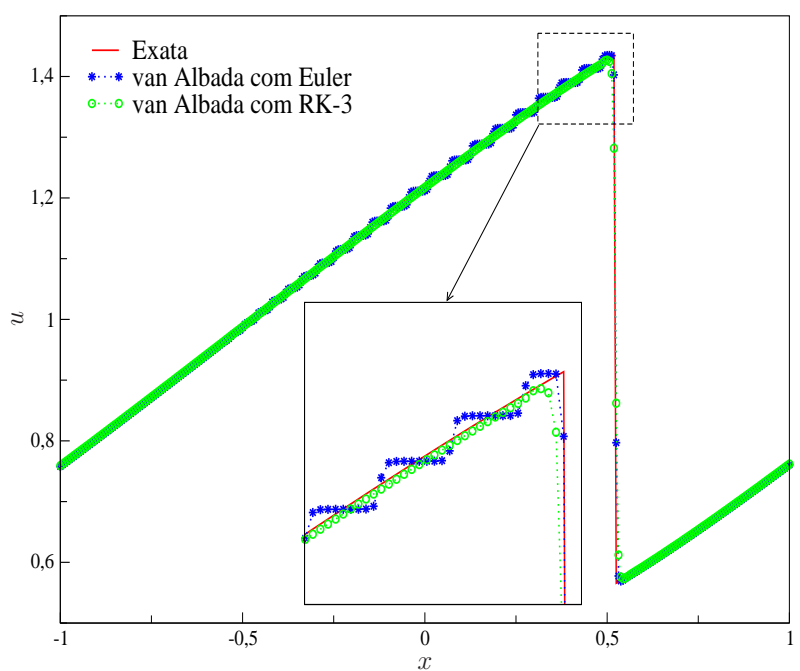

(d)

Figura 6.6: Comparação dos resultados numéricos obtidos para o problema da condição suave com os métodos de Euler explícito e RK-3 em $t=1.52$, usando 320 células computacionais e $\theta=0.4$.

\section{Problema 5: (Ponto sônico)}

Neste teste a atenção é voltada a análise dos esquemas na captura do ponto sônico. A condição inicial e a solução exata para esse teste são (ver [35]), respectivamente, dadas por

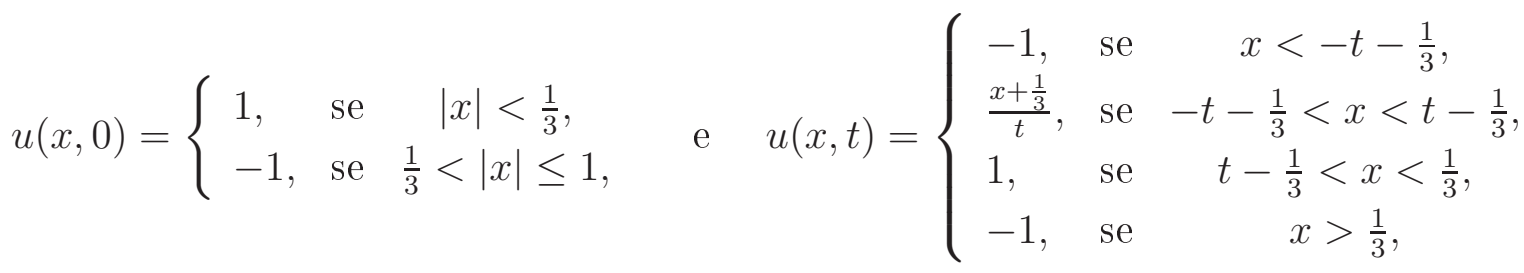

e as condições de contorno são periódicas. Essa condição inicial descreve uma onda quadrada 
que consiste em um salto de -1 a 1 , em $x=-\frac{1}{3}$, que produz uma onda de rarefação, enquanto o salto de 1 a -1 , em $x=\frac{1}{3}$, cria uma onda de choque. O ponto sônico aparece quando $F^{\prime}(u)=u=0$, ou seja, quando a velocidade $u$ da onda muda de sinal ( $u$ varia de -1 a 1 ). Nesta região dificuldades numéricas podem surgir em que um extremo local é criado e a solução não é monótona ao longo da onda rarefação. Para solucionar esse problema e fornecer uma transição suave em torno do ponto sônico, uma correção de entropia adequada é usada para adicionar mais viscosidade numérica no esquema de diferença finita (5.1), isto é,

$$
\phi_{i}^{n+1}=\phi_{i}^{n}-\theta\left(F(\phi)_{i+1}^{n}-F(\phi)_{i-1}^{n}\right)+\frac{Q_{i+\frac{1}{2}}}{2}\left(\phi_{i+1}^{n}-\phi_{i}^{n}\right)-\frac{Q_{i-\frac{1}{2}}}{2}\left(\phi_{i}^{n}-\phi_{i-1}^{n}\right),
$$

em que o termo $Q_{i+\frac{1}{2}}$ é dado pela correção de entropia de Harten [26] da forma

$$
Q(x)= \begin{cases}|x|, & |x|>\varepsilon \\ \frac{x^{2}+\varepsilon^{2}}{2 \varepsilon}, & |x| \leq \varepsilon\end{cases}
$$

sendo $\varepsilon=0.5$ uma constante positiva.

Para a simulação desse problema são usados uma malha de 200 células computacionais, $\theta=0.7$ e $t=0.3$. A Figura 6.7 mostra a comparação entre a solução exata e as soluções numéricas geradas pelo esquema PFDPUS (para diferentes pares de parâmetros livres $\alpha$ e $\beta$ ). Pode-se notar, que tanto com ou sem correção de entropia o choque é perfeitamente capturado pelo esquema PFDPUS com qualquer um dos parâmetros livres escolhidos. No entanto, este esquema falha na captura da onda de rarefação gerando uma expansão não física da onda quando a correção de entropia não é aplicada. Ao implementar a correção de entropia, o esquema PFDPUS apresenta resultados semelhantes para qualquer um dos pares de parâmetros livres, obtendo soluções suaves e em boa concordância com a solução exata na região onde o ponto sônico se encontra.

A Figura 6.8 mostra a comparação entre a solução exata e as soluções numéricas geradas pelos esquemas PFDPUS ( $\alpha=10$ e $\beta=100$ ), ADBQUICKEST, SDPUS-C1 e Superbee. Observa-se que todos os esquemas geram uma expansão não física da onda rarefação quando não se aplica a correção de entropia. Em compensação, ao aplicar a correção, os esquemas apresentam boa concordância com a solução exata, conseguindo capturar bem a solução nas vizinhanças do ponto sônico. Observa-se claramente que os esquemas PFDPUS e SDPUS-C1 geram os melhores resultados. Além disso, vê-se que o esquema ADBQUICKEST introduz considerável dissipação numérica por volta das quinas, no começo e no fim da onda rarefação.

\section{Problema 6: (Problema de Platzman)}

Neste teste não linear analisa-se as restrições TVD para o esquema PFDPUS (com os pares de parâmetros livres $\alpha=0$ e $\beta=-38, \alpha=3$ e $\beta=100, \alpha=9$ e $\beta=88$, e, $\alpha=10$ e $\beta=100$ ) 


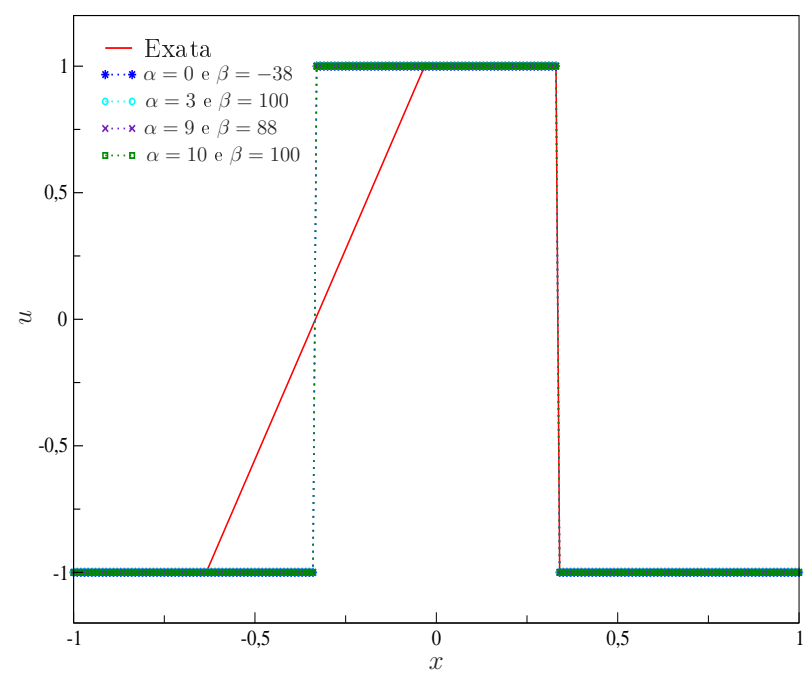

(a) Sem correção de entropia

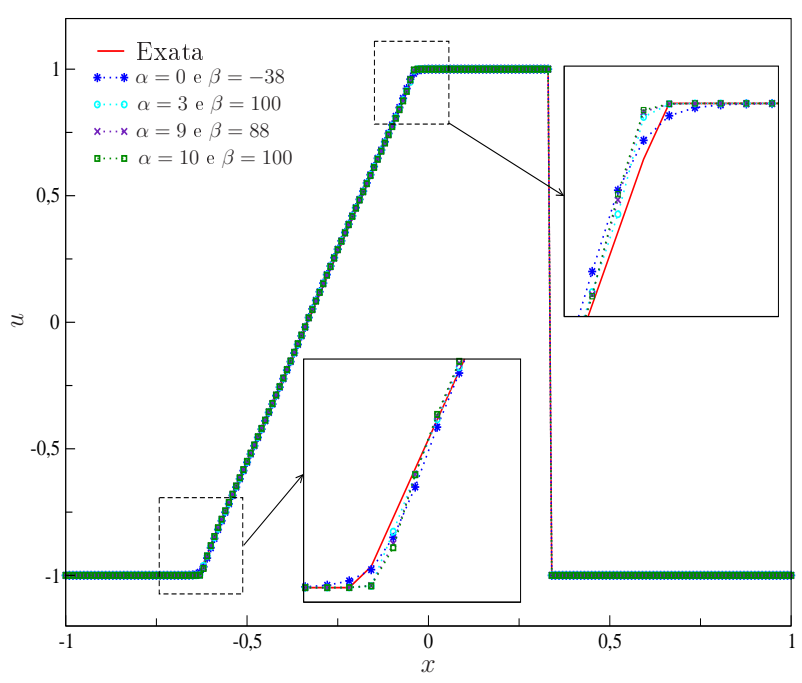

(b) Com correção de entropia

Figura 6.7: Resultados numéricos obtidos para o problema do ponto sônico com o esquema PFDPUS em $t=0.3$, usando 200 células computacionais e $\theta=0.7$.

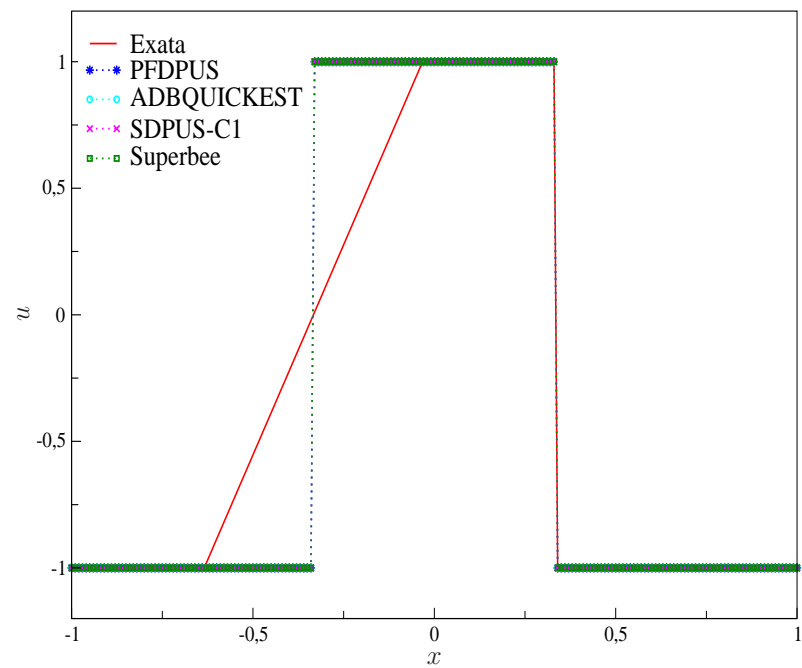

(a) Sem correção de entropia

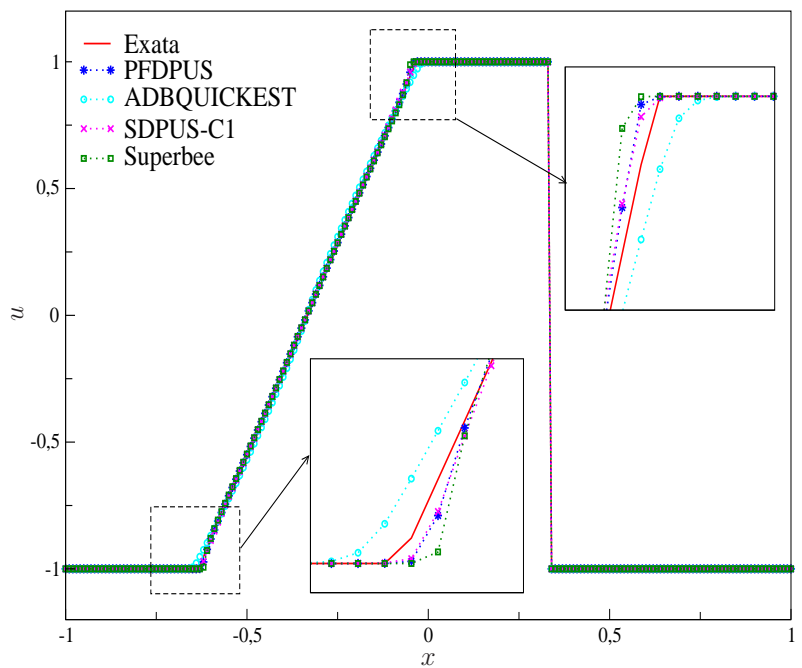

(b) Com correção de entropia

Figura 6.8: Resultados numéricos obtidos para o problema do ponto sônico em $t=0.3$, usando 200 células computacionais e $\theta=0.7$.

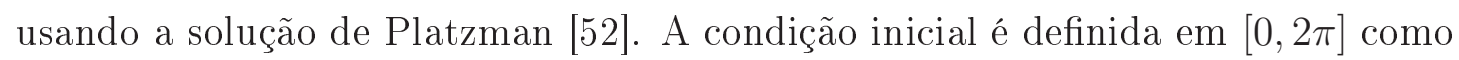

$$
u(x, 0)=\operatorname{sen}(x),
$$

e as condições de contorno são periódicas. Esse problema tem solução exata dada da forma (ver Platzman [52])

$$
u(x, t)=-2 \sum_{m}^{\infty} \frac{J_{m}(-m t)}{m t} \operatorname{sen}(m x),
$$

em que $J_{m}$ é função de Bessel. Esta solução é válida para os tempos anteriores ao choque, 
que ocorre em $t=1$. Para calcular a solução exata, a série (6.9) é truncada em $m=200$. Para analisar a restrição TVD, calcula-se a cada passo de tempo, a TV dada por (3.6) e plotam-se os resultados no plano $T V(u(t)) \perp t$. Para isso, consideram-se 300, 600 e 900 células computacionais, $\theta=0.4$ e $t=0.25$ (antes do choque).

A Figura 6.9 mostra a TV exata $(T V(u(t))=4)$ e as TVs numéricas obtidas usando o esquema PFDPUS (com os diferentes pares de parâmetros livres $\alpha$ e $\beta$ ). Nota-se claramente por essa figura que as TVs associadas ao esquema PFDPUS satisfazem a restrição TVD, para qualquer par de parâmetros livres, uma vez que todas as TVs numéricas apresentam comportamentos decrescentes. Além disso, observa-se que ao se refinar a malha as TVs numéricas aproximam-se cada vez mais da TV exata. Os melhores resultados obtidos com o esquema PFDPUS nesse problema não linear ocorrem com a escolha dos parâmetros $\alpha=10$ e $\beta=100$.

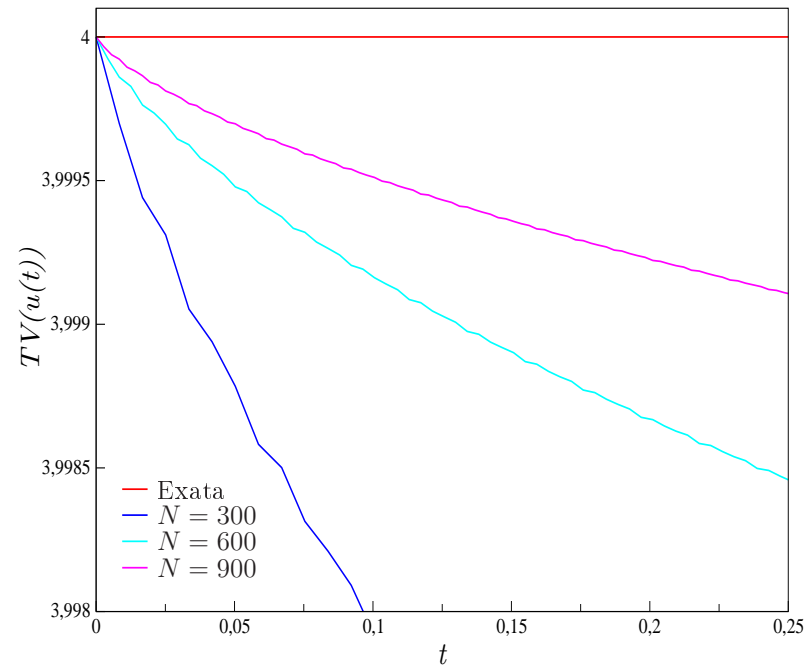

(a) $\alpha=0$ e $\beta=-38$

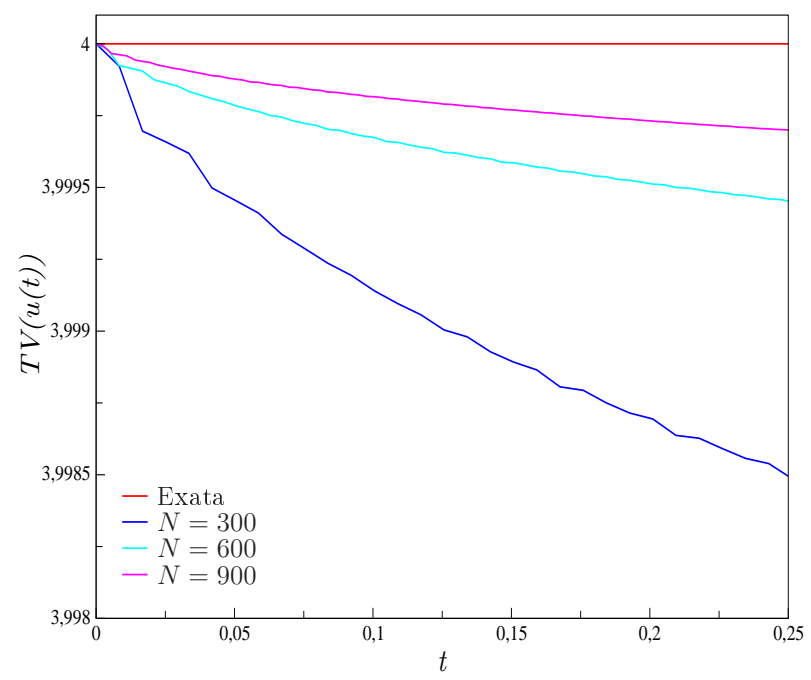

(c) $\alpha=9$ e $\beta=88$

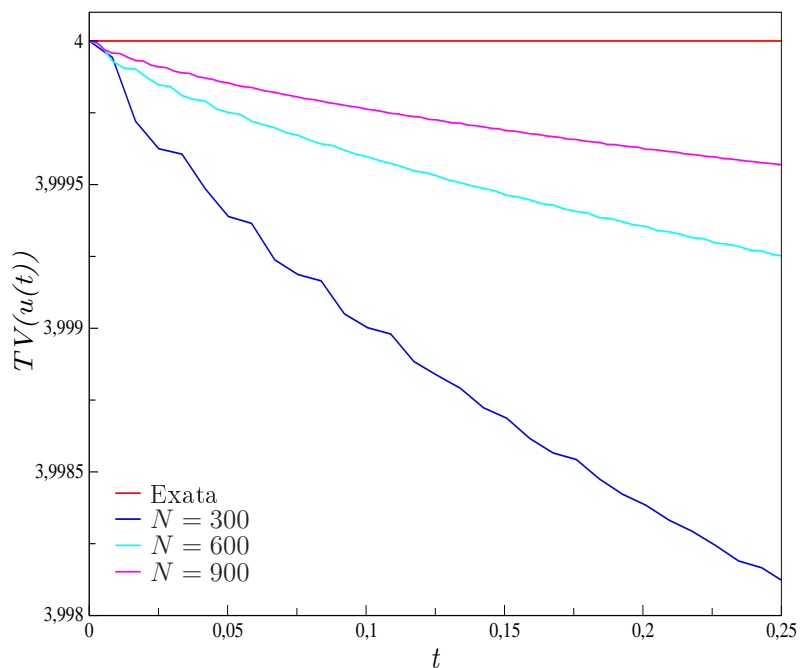

(b) $\alpha=3$ e $\beta=100$

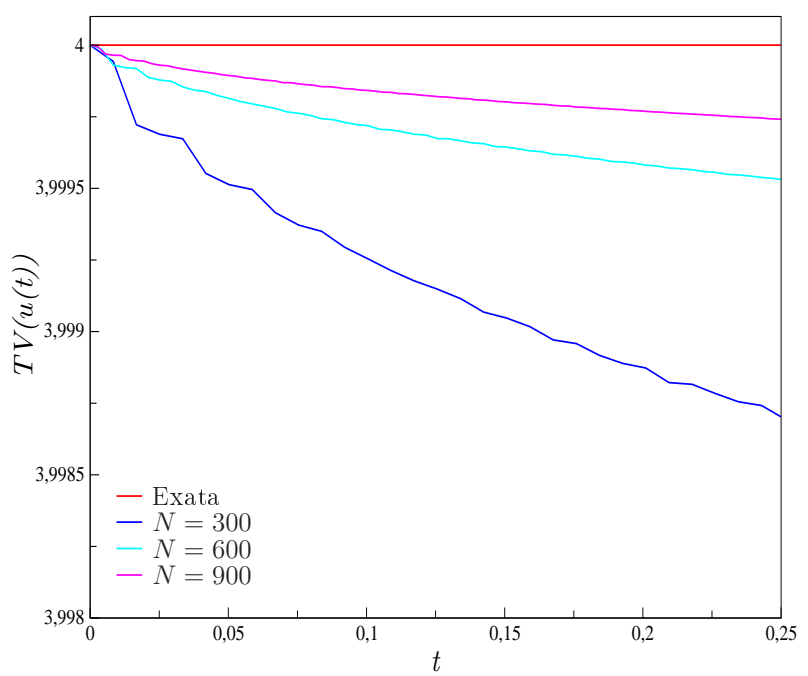

(d) $\alpha=10$ e $\beta=100$

Figura 6.9: Análise da restrição TVD para o problema de Platzman usando o esquema PFDPUS com os parâmetros livres $\alpha$ e $\beta$ em $t=0.25$. 


\subsection{Equações de Euler}

Nesta seção são apresentados resultados numéricos para as equações de Euler que são formuladas pela lei de conservação 1D (2.1), com vetor de variáveis conservadas (2.12) e vetor da função fluxo (2.13). As simulações numéricas são realizadas com o auxílio do pacote computacional CLAWPACK ${ }^{1}$ equipado com os limitadores de fluxo dos esquemas PFDPUS (com todos os pares de parâmetros livres $\alpha$ e $\beta$ adotados), ADBQUICKEST, TOPUS, SDPUS-C1 e van Albada. As comparações entre os resultados numéricos são feitas com base em soluções de referência geradas no CLAWPACK por meio do método de Godunov de primeira ordem, com termo de correção, onde o limitador de fluxo do esquema MC é implementado. Mais detalhes sobre esse pacote computacional podem ser encontrados no Apêndice B e em LeVeque [43].

\section{Problema 7: (Problema do tubo de choque de Sod)}

Este problema consiste de um tubo unidimensional contendo dois gases, com diferentes densidades e pressões, separados por uma membrana na posição $x=0.4$. As duas regiões estão em estado constante e os gases estão inicialmente em repouso. Em $t=0$ a membrana é quebrada e os dois gases se interagem, gerando uma onda de rarefação que se propaga para a esquerda, uma descontinuidade de contato e uma onda de choque viajando para a direita (ver detalhes em Sod [60] e LeVeque et al. [45]). Em resumo, os dados iniciais são definidos em [0, 1] da forma

$$
\left[\rho_{0}, u_{0}, P_{0}\right]^{T}= \begin{cases}{[1,0,1]^{T},} & \text { se } x<0.4, \\ {[0.125,0,0.1]^{T},} & \text { se } x \geq 0.4,\end{cases}
$$

e as condições contorno são dadas por extrapolação de ordem zero. Os dados utilizados nas simulações são malha de 400 células computacionais, $\theta=0.8$ e $t=0.2$. O cálculo das soluções numéricas são feitos usando-se o método de Godunov de primeira ordem, com termo de correção, aplicando-se os limitadores de fluxo dos esquemas PFDPUS (com os diferentes pares de parâmetros livres $\alpha$ e $\beta$ adotados), ADBQUICKEST, TOPUS, SDPUS-C1 e van Albada. Para a solução de referência considera-se o método de Godunov de primeira ordem com termo de correção, em que adota-se o limitador de fluxo MC, malha de 2000 células computacionais e $\theta=0.1$.

A Figura 6.10 mostra as comparações entre as soluções de referência e numéricas obtidas com o esquema PFDPUS (com os diferentes pares de parâmetros livres $\alpha$ e $\beta$ ) para a densidade, velocidade e pressão. Observa-se que os resultados numéricos obtidos com o esquema PFDPUS, em geral, são satisfatórios quando comparados com a solução de referência. Além disso, a partir das ampliações, constata-se que o par de parâmetros livres $\alpha=10$ e $\beta=100$ apresenta a melhor aproximação.

As Figuras 6.11 e 6.12 mostram, respectivamente, os resultados numéricos (e suas ampliações) com os esquemas PFDPUS ( $\alpha=10$ e $\beta=100$ ), ADBQUICKEST, TOPUS, SDPUS-C1

\footnotetext{
${ }^{1}$ http://www.amath.washington.edu/ claw/
} 


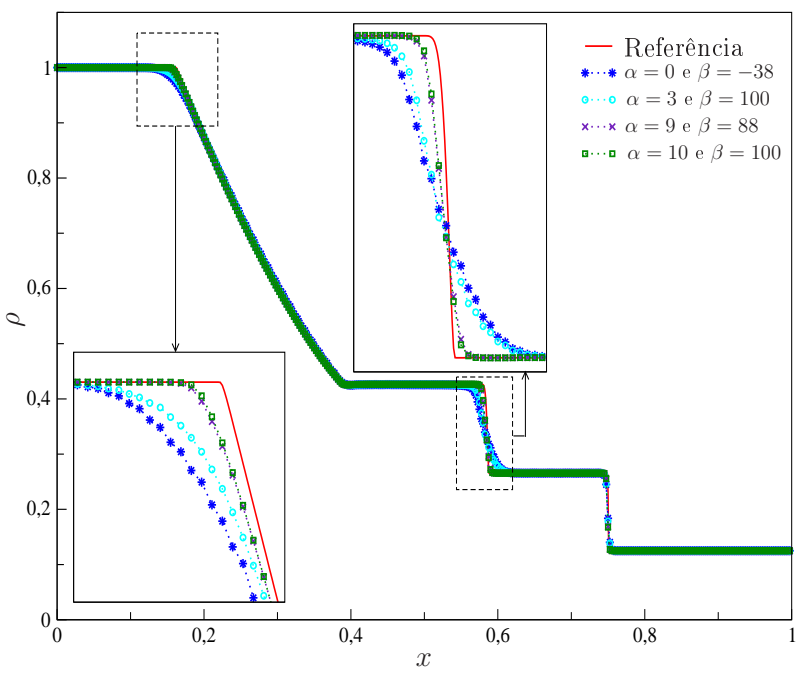

(a) Densidade

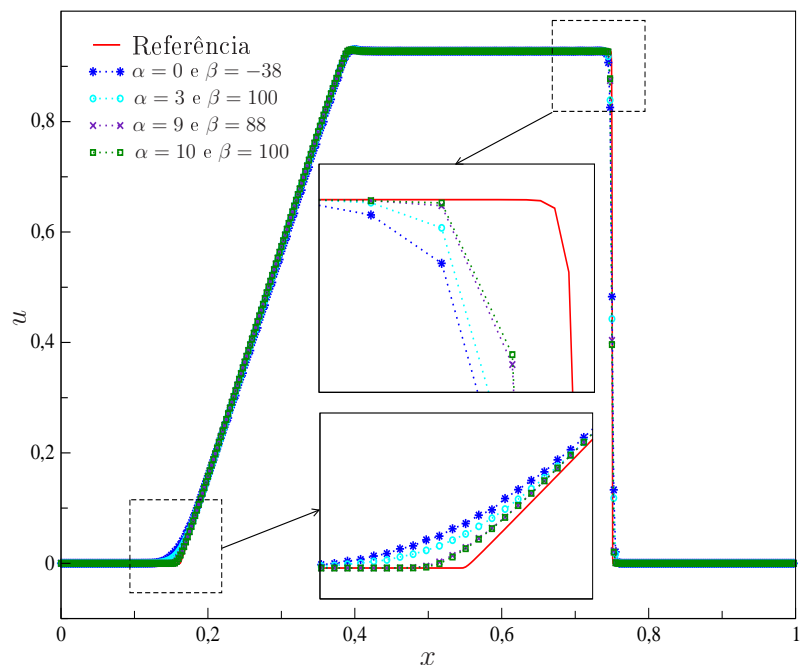

(b) Velocidade

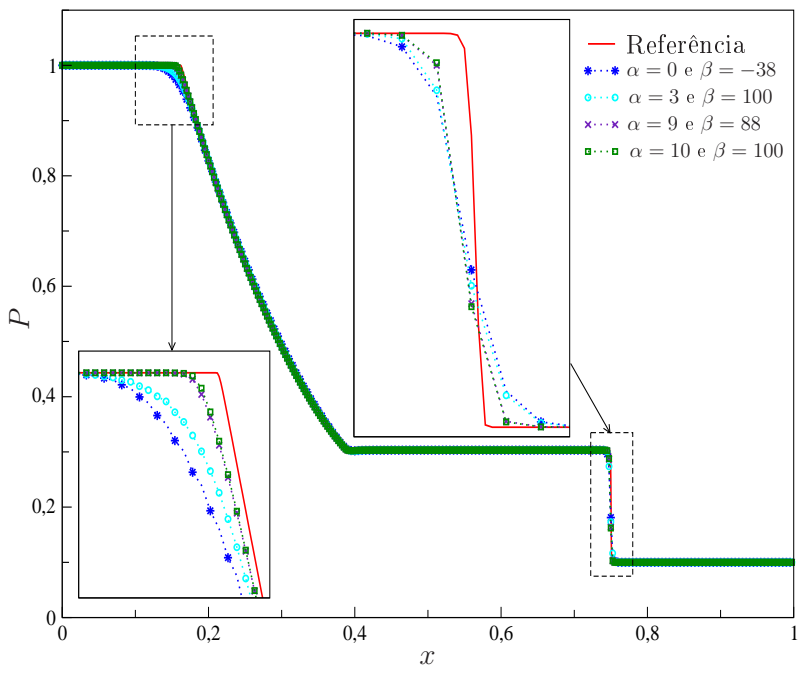

(c) Pressão

Figura 6.10: Resultados numéricos obtidos para o problema do tubo de choque de Sod com o esquema PFDPUS em $t=0.2$, usando 400 células computacionais e $\theta=0.8$.

e van Albada obtidos para a densidade e pressão. Por meio dessas figuras, vê-se que todos os esquemas apresentam resultados satisfatórios, sendo que o esquema PFDPUS $(\alpha=10$ e $\beta=100)$ fornece, no geral, resultados superiores.

\section{Problema 8: (Ondas com fortes colisões)}

Este problema é usualmente conhecido na literatura por Interacting Blast Waves Problem devido a Woodward e Colella [79] e é bastante interessante, pois testa a habilidade do esquema numérico em fornecer soluções não oscilatórias na presença de fortes choques e complexas interações entre ondas. O problema envolve um escoamento em um tubo paralelo que se estende de 0 a 1 fechado nas extremidades. Em $t=0$, o tubo contém um gás ideal com densidade 


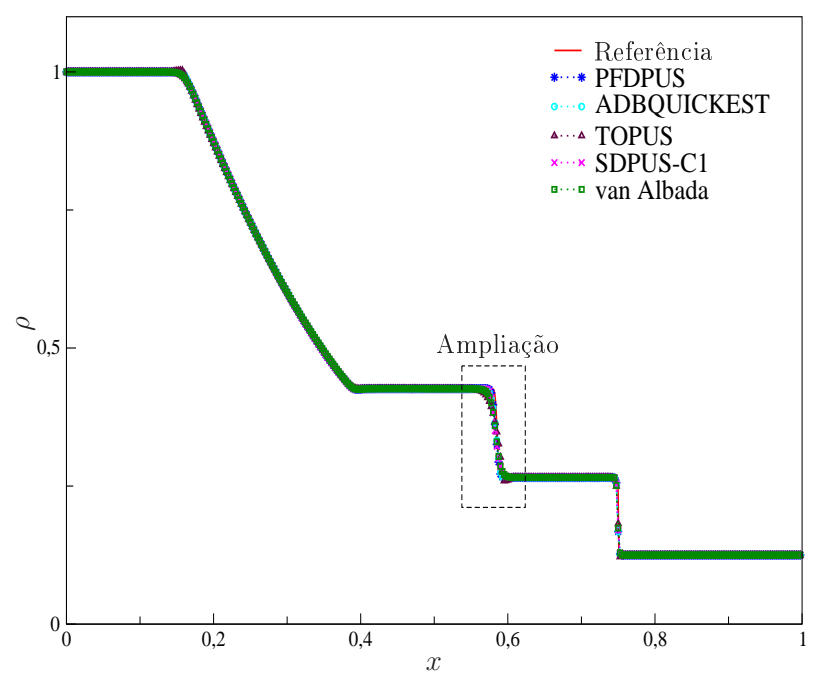

(a) Comparação dos resultados numéricos

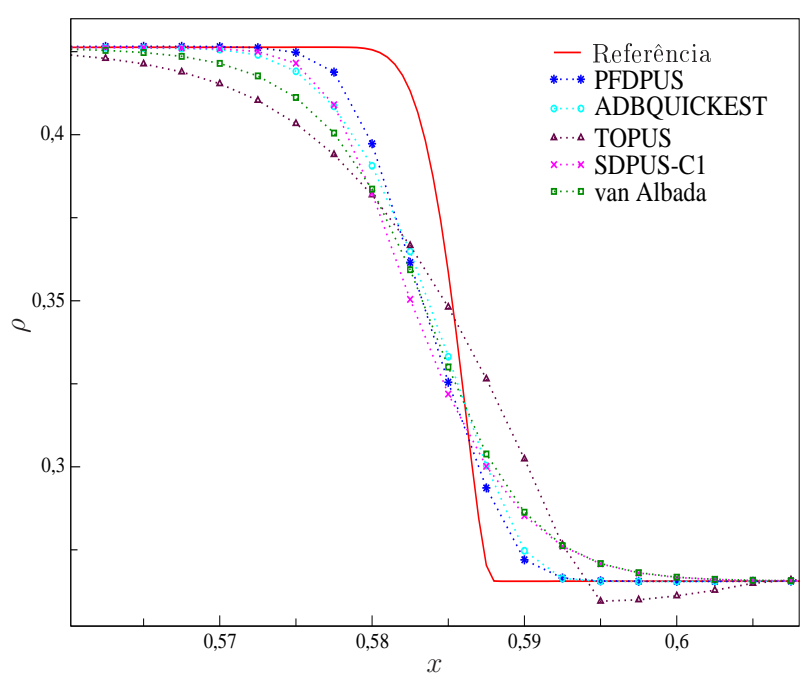

(b) Ampliação

Figura 6.11: Resultados numéricos do problema do tubo de choque de Sod para densidade em $t=0.2$, usando 400 células computacionais e $\theta=0.8$.

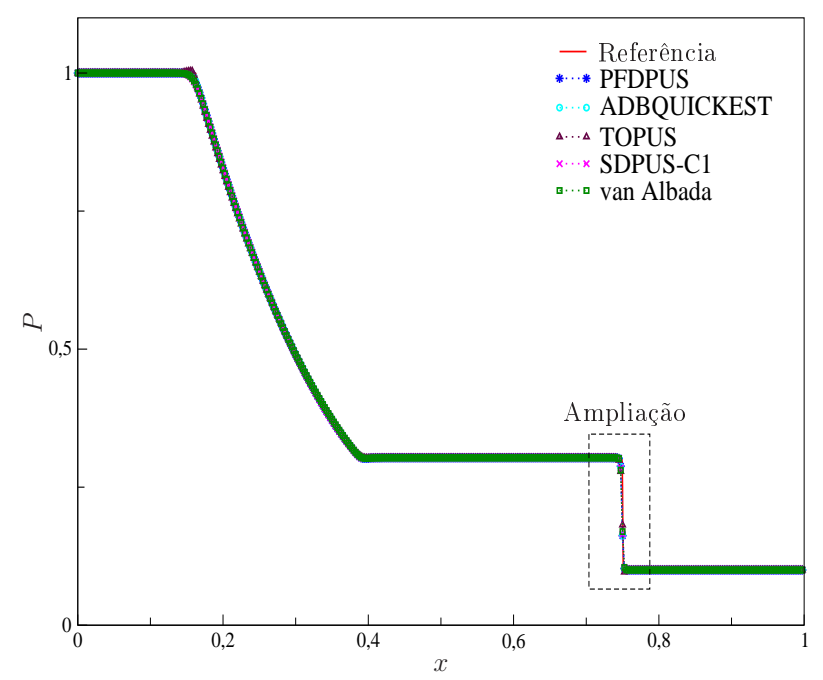

(a) Comparação dos resultados numéricos

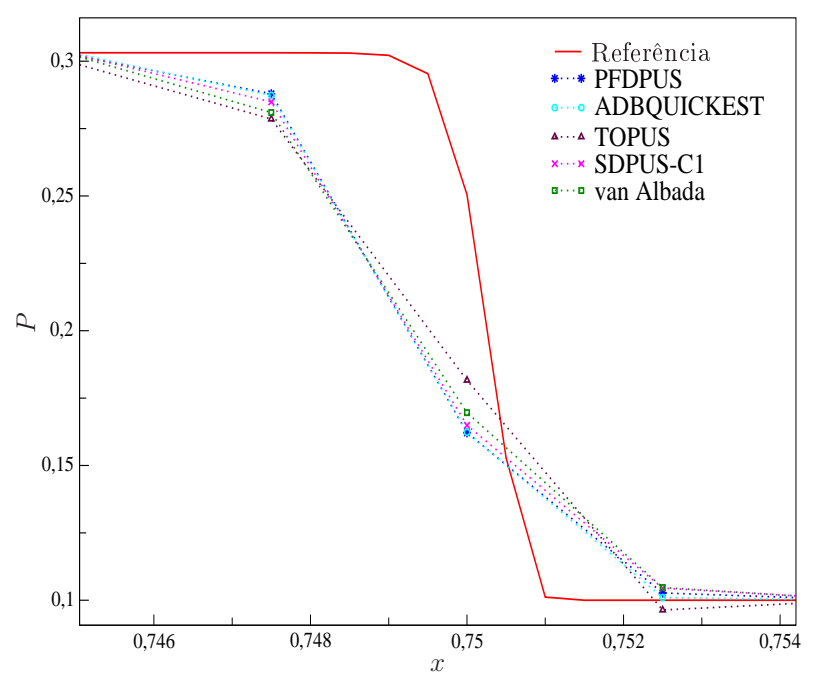

(b) Ampliação

Figura 6.12: Resultados numéricos do problema do tubo de choque de Sod para pressão em $t=0.2$, usando 400 células computacionais e $\theta=0.8$.

unitária em todos os lugares e em repouso. No entanto, duas membranas em $x=0.1$ e $x=0.9$ separam as três regiões com diferentes pressões. Essas membranas se rompem simultaneamente e fortes ondas de choque surgem na região central, produzindo um fluxo complexo. Os dados inicias são definidos em $[0,1]$ como

$$
\left[\rho_{0}, u_{0}, P_{0}\right]^{T}=\left\{\begin{array}{llc}
{[1,0,1000]^{T},} & \text { se } & 0 \leq x \leq 0.1 \\
{[1,0,0.01]^{T},} & \text { se } & 0.1<x \leq 0.9 \\
{[1,0,100]^{T},} & \text { se } & 0.9<x \leq 1,
\end{array}\right.
$$


e as condições de contorno adotadas são reflexivas.

Inicialmente, com o objetivo de verificar a precisão dos esquemas PFDPUS $(\alpha=10$ e $\beta=$ 100), ADBQUICKEST, TOPUS e SDPUS-C1 neste problema não linear complexo, é calculada a ordem de convergência para a densidade. Para tanto, as soluções numéricas são computadas pelo método de primeira ordem de Godunov com termo de correção, aplicando-se os limitadores dos esquemas considerados, com malhas de 125, 250, 500 e 1000 células computacionais, $\theta=0.8$ e $t=0.038$. A solução de referência considerada aqui usa o limitador de fluxo MC no termo de correção $\operatorname{com} \theta=0.3$ e 2000 células computacionais.

Os resultados obtidos para os erros, nas normas $L_{1}$ e $L_{2}$, e as ordens de convergência estão mostrados na Tabela 6.7. Pode-se observar que, tanto para a norma $L_{1}$ quanto para a norma $L_{2}$, o esquema PFDPUS $(\alpha=10$ e $\beta=100$ ) apresenta ordem superior às ordens obtidas pelos demais esquemas. Em particular, na malha mais fina $(N=1000)$ a ordem do esquema PFDPUS é mais próxima de 2 que os outros esquemas. Além disso, nota-se que os esquemas ADBQUICKEST e SDPUS-C1 apresentam ordens semelhantes, sendo que o TOPUS fornece, no geral, a ordem mais baixa.

\begin{tabular}{lcccccc}
\hline Esquemas & $N$ & \multicolumn{2}{c}{$L_{1}$} & \multicolumn{2}{c}{$L_{2}$} \\
\cline { 3 - 4 } \cline { 6 - 7 } & & $\left\|E_{h}\right\|_{1}$ & $p$ & & $\left\|E_{h}\right\|_{2}$ & $p$ \\
\hline PFDPUS & 125 & 0.218804 & - & & 0.690462 & - \\
& 250 & 0.080430 & 1.443839 & & 0.286873 & 1.267151 \\
& 500 & 0.029475 & 1.448253 & & 0.124104 & 1.208859 \\
ADBQUICKEST & 1000 & 0.008434 & 1.805133 & & 0.037223 & 1.737274 \\
& 250 & 0.237193 & - & & 0.668218 & - \\
& 500 & 0.103213 & 1.200440 & & 0.323595 & 1.046131 \\
TOPUS & 1000 & 0.016250 & 1.507161 & & 0.069611 & 1.325816 \\
& 125 & 0.260854 & - & & 0.754506 & - \\
& 250 & 0.117346 & 1.152473 & & 0.380290 & 0.988432 \\
SDPUS-C1 & 500 & 0.055640 & 1.076562 & & 0.210927 & 0.850354 \\
& 1000 & 0.020468 & 1.442732 & & 0.088034 & 1.260610 \\
& 125 & 0.247468 & - & & 0.746562 & - \\
& 250 & 0.105050 & 1.236174 & & 0.357689 & 1.061559 \\
& 500 & 0.046787 & 1.166902 & & 0.188455 & 0.924486 \\
& 1000 & 0.016156 & 1.533999 & & 0.073112 & 1.366034 \\
\hline
\end{tabular}

Tabela 6.7: Teste de convergência para o problema das ondas com fortes colisões em $t=0.038$.

As Figuras 6.13 e 6.14 mostram as comparações entre as soluções numéricas, obtidas com os esquemas PFDPUS ( $\alpha=10$ e $\beta=100$ ), ADBQUICKEST, TOPUS e SDPUS-C1, e de referência para densidade e pressão, respectivamente. Nessas figuras, todas as soluções numéricas são comparadas também entre si em um único gráfico (ver Figuras 6.13-(e)/(f) para densidade e a 
Figura 6.14-(e)/(f) para a pressão). As soluções numéricas são geradas em uma malha de 500 células computacionais e a de referência com 2000 células. Pode-se perceber que os resultados são bastante satisfatórios. Em particular, o esquema PFDPUS apresenta uma solução mais próxima da solução de referência.

\subsection{Equações MHD}

As equações MHD são formuladas pela lei de conservação 1D (2.1), com vetor de variáveis conservadas e função fluxo dados, respectivamente, por (2.16) e (2.17). As simulações numéricas são computadas com o auxílio do pacote computacional CLAWPACK, equipado com os limitadores de fluxo dos esquemas PFDPUS ( $\alpha=10$ e $\beta=100)$, ADBQUICKEST, TOPUS, SDPUS-C1 e van Albada. Os resultados numéricos são comparados com soluções de referência geradas também com o CLAWPACK, onde no termo de correção o limitador de fluxo do esquema MC é aplicado. Para mais detalhes do pacote computacional CLAWPACK ver Apêndice B e LeVeque [43].

\section{Problema 9: (Problema de Riemann por Brio e Wu)}

Para o estudo das equações MHD é escolhido um problema de Riemann, como descrito em Brio e Wu [7]. Esse é um problema composto por dados iniciais que consistem em dois estados constantes onde a descontinuidade é localizada no meio do intervalo computacional. Os dados iniciais são definidos em $[-1,1]$ da forma

$$
\left[\rho_{0}, u_{0}, v_{0}, w_{0}, B_{2}^{0}, B_{3}^{0}, P_{0}\right]^{T}= \begin{cases}{[1,0,0,0,1,0,1]^{T},} & \text { se } x<0 \\ {[0.125,0,0,0,-1,0,0.1]^{T},} & \text { se } x \geq 0\end{cases}
$$

e as condições de contorno são dadas por extrapolação de ordem zero. Além disso é considerado $B_{1} \equiv 0.75$, em virtude da condição de divergência livre para o campo magnético (ver Seção (2.1.4)). Este problema constitui um teste interessante para verificar o desempenho de esquemas numéricos, uma vez que sua solução apresenta ricas estruturas que envolvem ondas de choque, ondas de rarefação e ondas compostas (choque imediato seguido por uma rarefação). As soluções numéricas são obtidas em $t=0.2$ numa malha de 500 células computacionais, $\theta=0.8$, pelo método de primeira ordem de Godunov com termo de correção, em que os limitadores de fluxo dos esquemas PFDPUS ( $\alpha=10$ e $\beta=100$ ), ADBQUICKEST, TOPUS, SDPUS-C1 e van Albada são implementados. A solução de referência é gerada em uma malha com 2000 células computacionais também usando o método de primeira ordem de Godunov, com termo de correção, adotando-se limitador de fluxo $\mathrm{MC}$ e $\theta=0.3$.

A Figura 6.15 apresenta os resultados numéricos obtidos para densidade, velocidade na direção $x$ e pressão, respectivamente. Pode-se notar que, apesar das estruturas complexas que são formadas na solução, os esquemas estudados são livres de oscilações não físicas, mostrando boa concordância com a solução de referência. E ainda mais, a partir das ampliações vê-se que o 


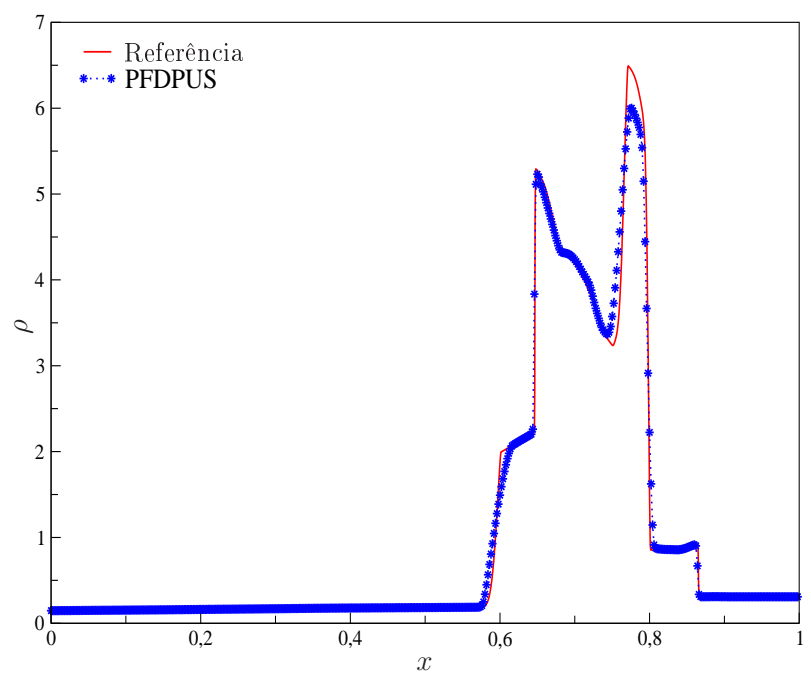

(a)

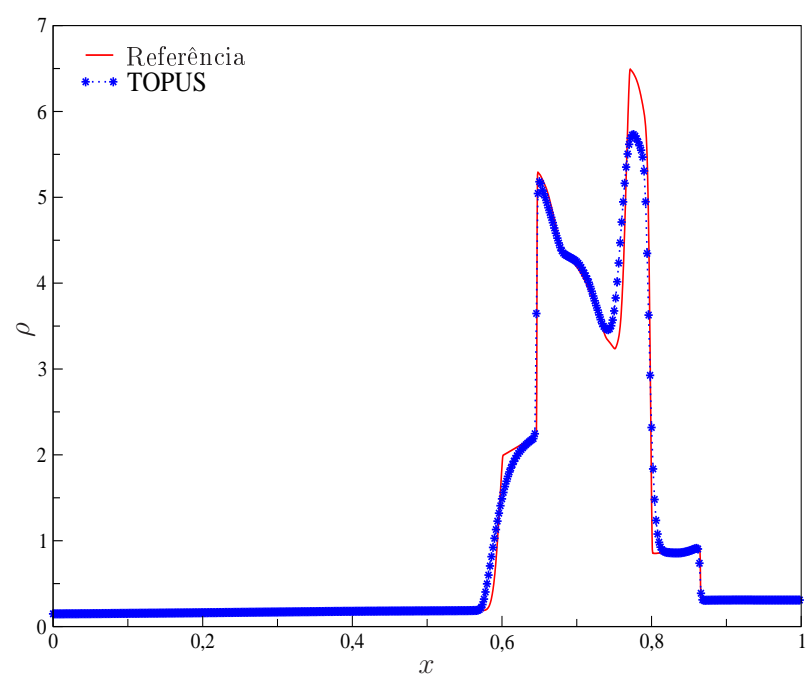

(c)

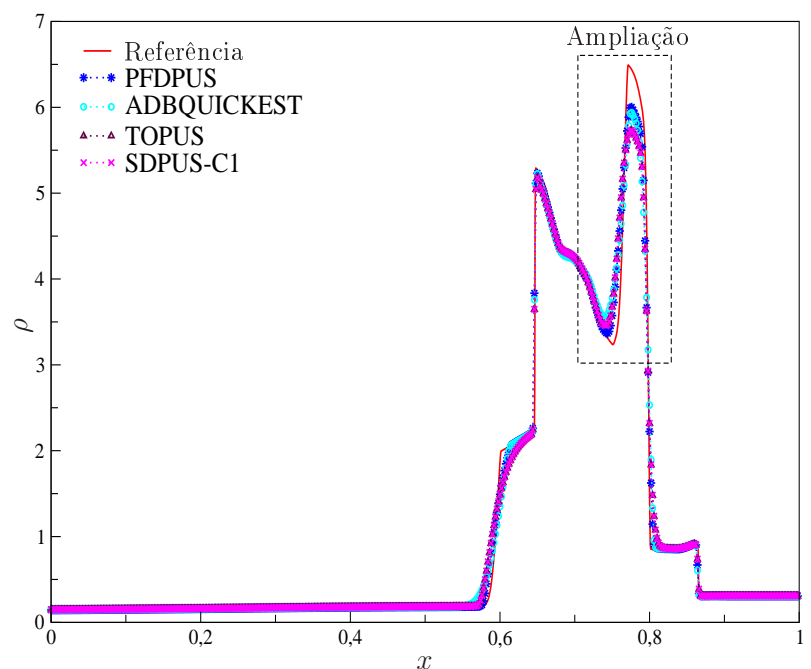

(e) Comparação dos resultados numéricos

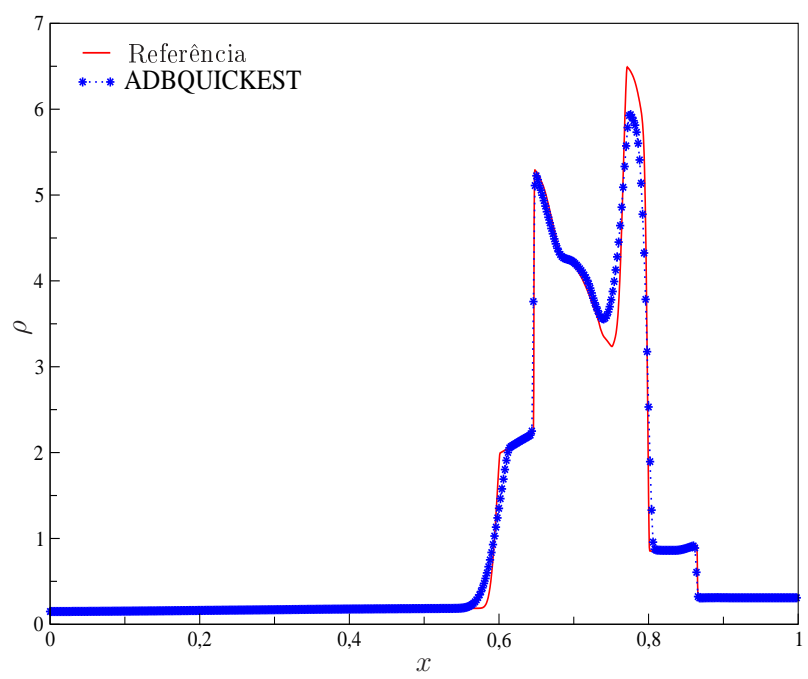

(b)

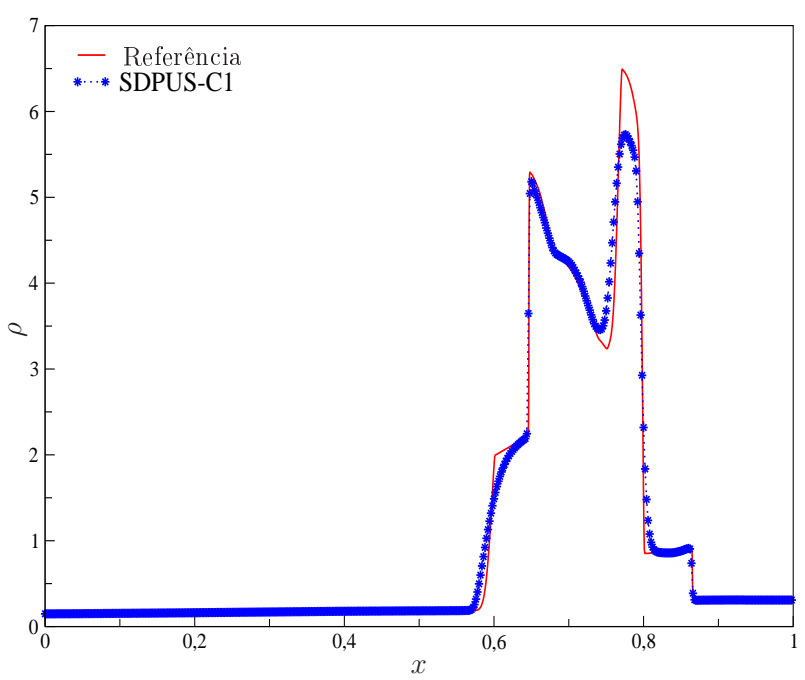

(d)

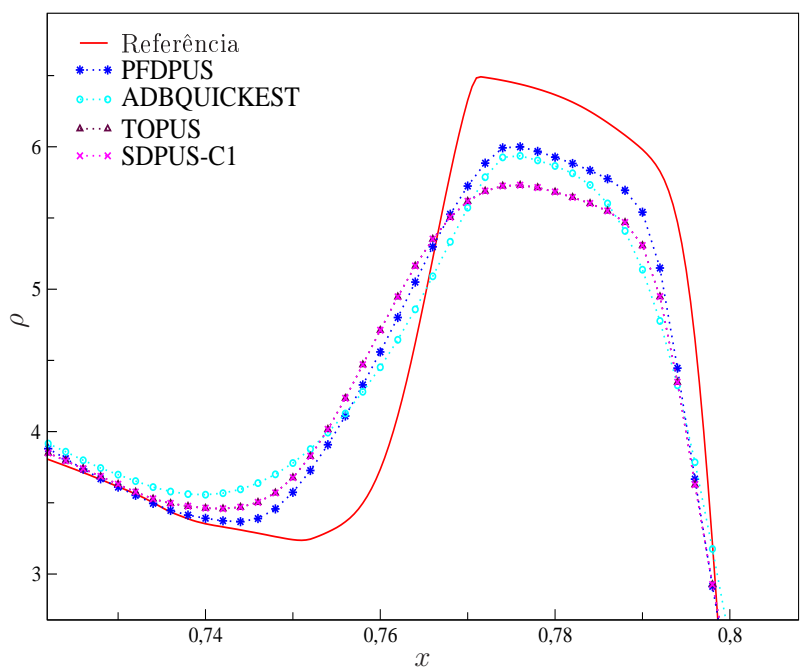

(f) Ampliação

Figura 6.13: Resultados numéricos do problema das ondas com fortes colisões para a densidade em $t=0.038$, usando 500 células computacionais e $\theta=0.8$. 


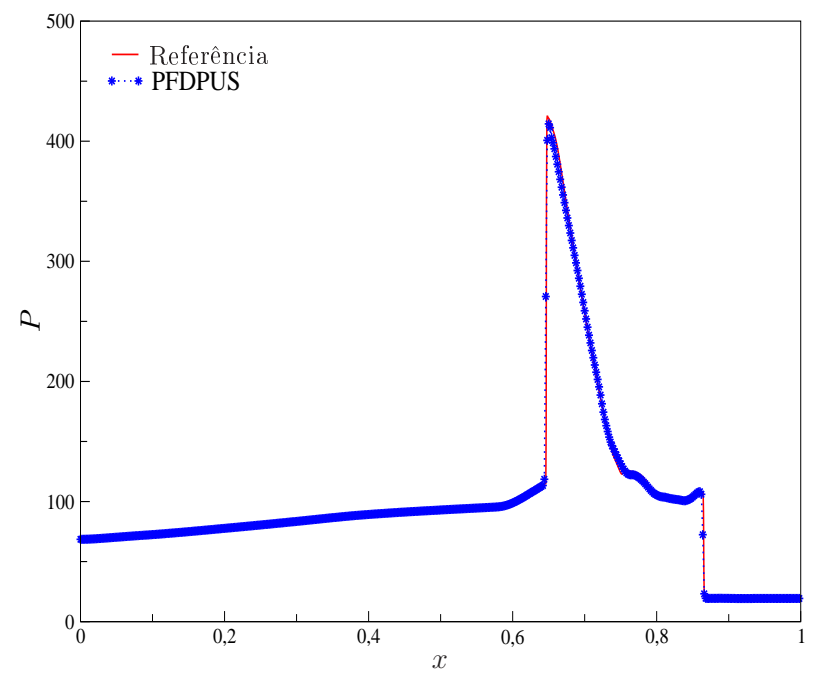

(a)

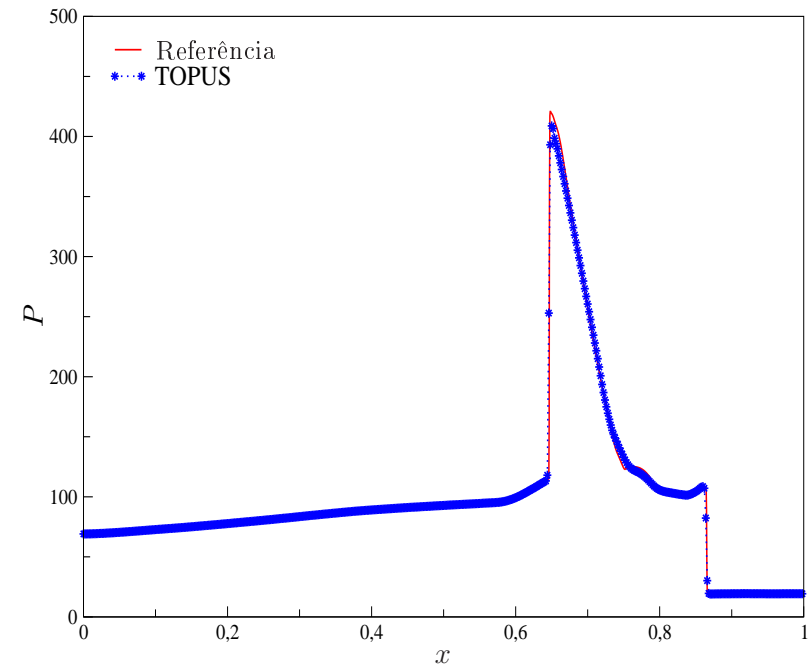

(c)

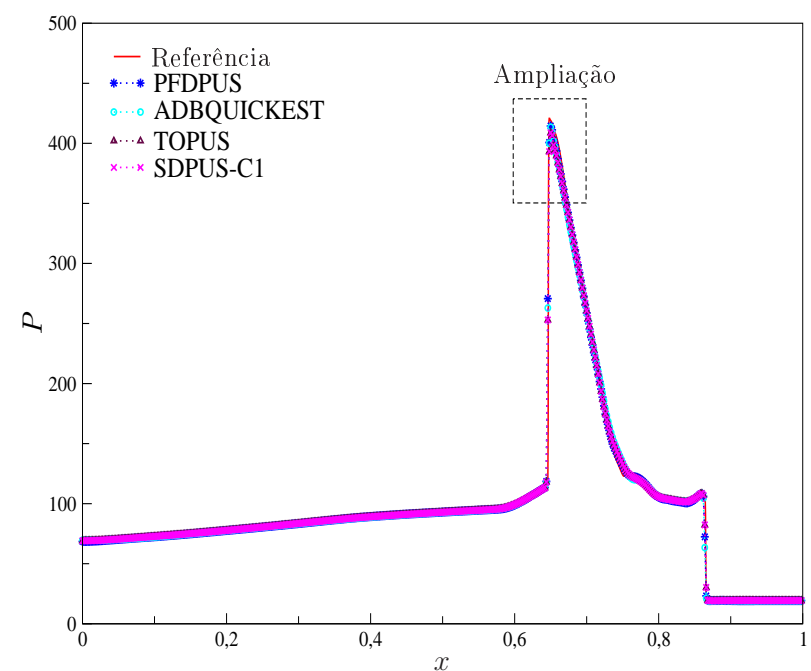

(e) Comparação dos resultados numéricos

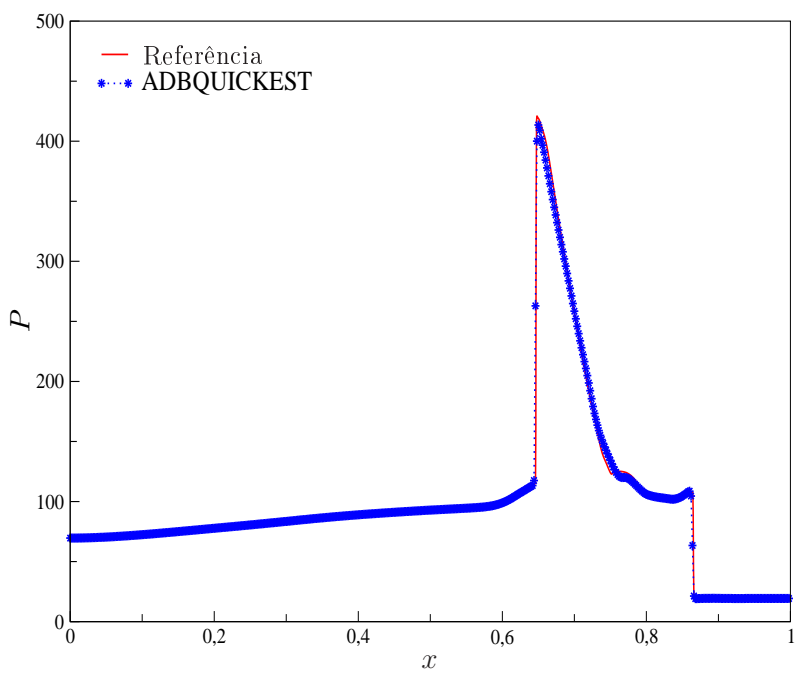

(b)

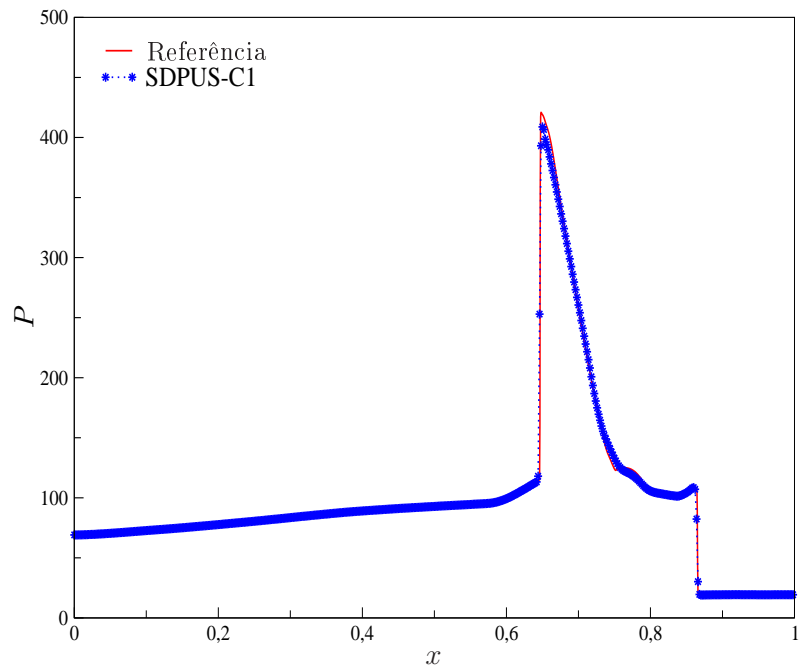

(d)

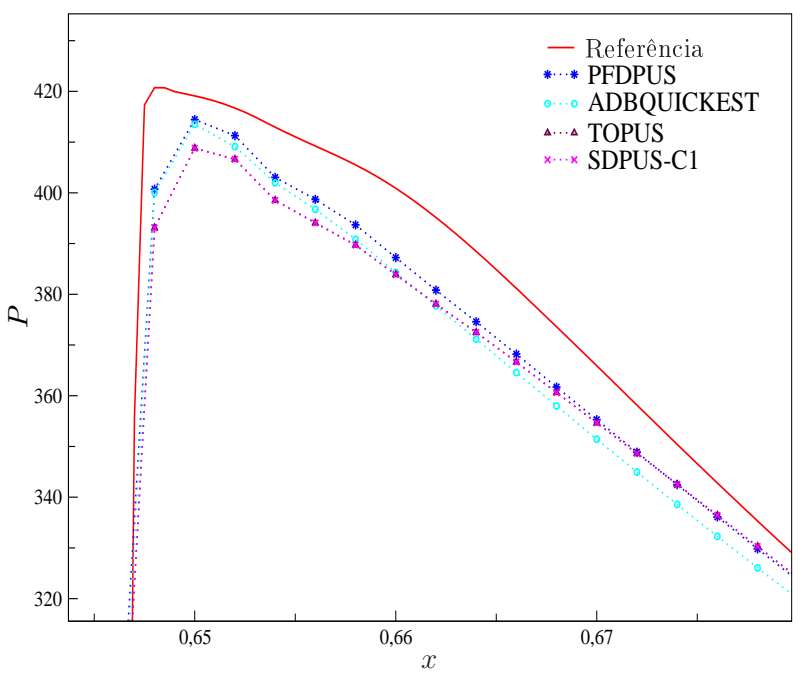

(f) Ampliação

Figura 6.14: Resultados numéricos do problema das ondas com fortes colisões para a pressão em $t=0.038$, usando 500 células computacionais e $\theta=0.8$. 
esquema PFDPUS apresenta desempenho igual ou superior aos demais esquemas considerados.

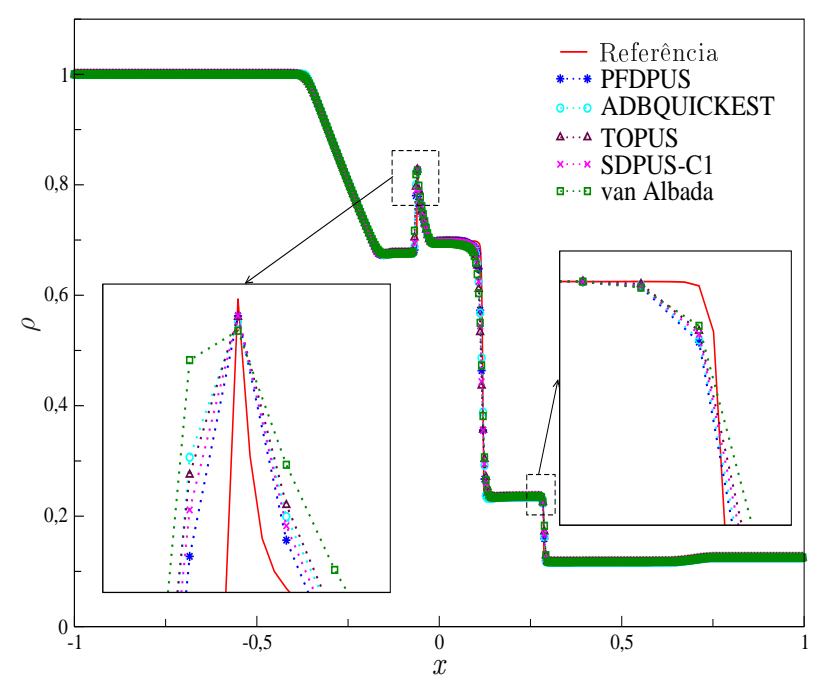

(a) Densidade

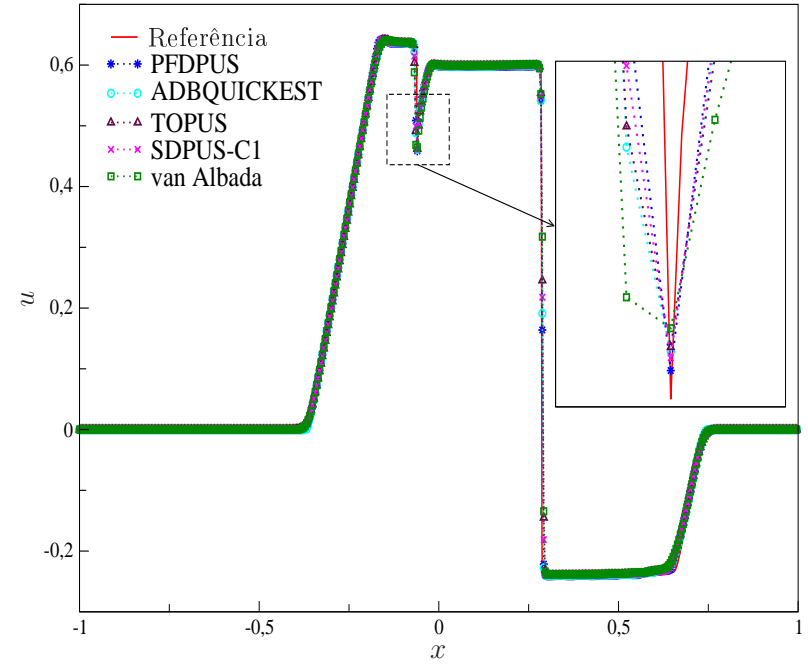

(b) Velocidade em $x$

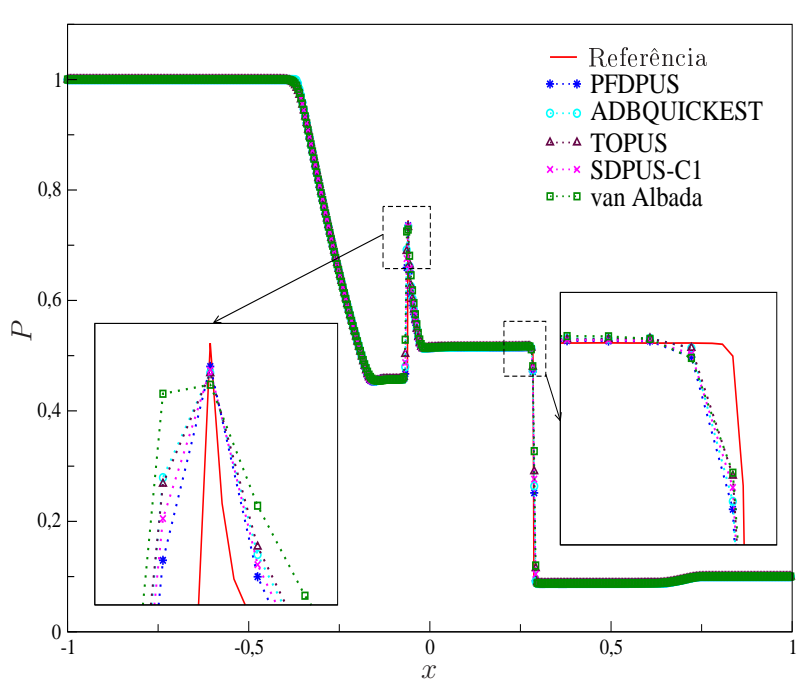

(c) Pressão

Figura 6.15: Resultados numéricos do problema de Riemann de Brio e Wu em $t=0.2$, usando 500 células computacionais e $\theta=0.8$.

\subsection{Comentários adicionais}

Neste capítulo, o esquema PFDPUS com parâmetros livres $\alpha=0$ e $\beta=-38, \alpha=3$ e $\beta=100, \alpha=9$ e $\beta=88$, e, $\alpha=10$ e $\beta=100$ foi utilizado com sucesso para resolver numericamente uma variedade de condições iniciais diferentes para as equações de advecções e de Burgers, e problemas de Riemann 1D para as equações de Euler e MHD. De maneira geral, os resultados numéricos mostram que esse novo esquema uwpind de alta resolução tem 
desempenho comparável com os demais esquemas estudados, mostrando ser capaz de controlar oscilações numéricas próximas as descontinuidades e introduzir pouca dissipação numérica. É importante ressaltar que, em geral, o esquema PFDPUS com parâmetros livres $\alpha=10$ e $\beta=100$ apresentou melhores resultados. Por essa razão, esse par de parâmetros livres foi escolhido para simular problemas multidimensionais presentes nos próximos capítulos. 
CAPÍTULO 7

\section{Resultados numéricos $2 D$ e axissimétricos}

Este capítulo apresenta os resultados numéricos para problemas 2D e axissimétricos com objetivo de investigar o desempenho do esquema PFDPUS em problemas mais complexos. Resolve-se leis de conservação 2D tais como as equações de águas rasas, Euler e MHD. Em seguida, apresentam-se resultados numéricos para escoamentos incompressíveis com superfícies livres móveis modelados pelas equações de Navier-Stokes. Para simulação de leis de conservação 2D e escoamentos incompressíveis são utilizados, respectivamente, o pacote computacional CLAWPACK [43] e o ambiente de simulação Freeflow [9]. Vale ressaltar que, nesse capítulo, os resultados numéricos obtidos com o esquema PFDPUS são gerados adotando-se o par de parâmetros livres $\alpha=10$ e $\beta=100$.

\subsection{Leis de conservação 2D}

Nessa seção são apresentados os resultados numéricos para as leis de conservação 2D como as equações de águas rasas, Euler e MHD. Para tanto, o pacote computacional CLAWPACK de LeVeque [43] é equipado com os limitadores de fluxo dos esquemas PFDPUS, ADBQUICKEST, TOPUS e SDPUS-C1.

\subsubsection{Equações de águas rasas}

O sistema de equações de águas rasas é formulado pela lei de conservação 2D (2.21), possuindo vetor de variáveis conservadas (2.23) e funções fluxo (2.24) e (2.25).

\section{Problema 1: (Circular dam-break flow)}

Este problema simula o colapso de uma barreira circular e é útil para testar o desempenho de esquemas numéricos na resolução de descontinuidades simétricas com superfícies livres 2D. A condição inicial consiste em duas regiões com água separadas por uma barreira cilíndrica 
de raio $r$ centrada no meio do domínio computacional. A profundidade da água no interior da barreira é $h_{1}$ e fora da barreira é $h_{2}$. Quando a barreira é removida uma onda de choque translada radialmente, enquanto uma onda de rarefação se move para o interior [43]. A Figura 7.1 ilustra esse problema em $t=0$ (antes do colapso) e em $t>0$ (depois do colapso).

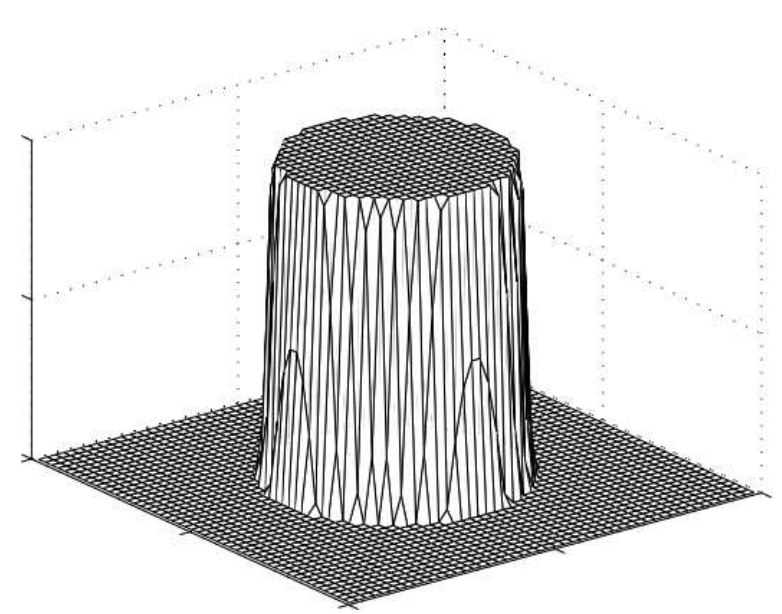

(a) Antes do colapso

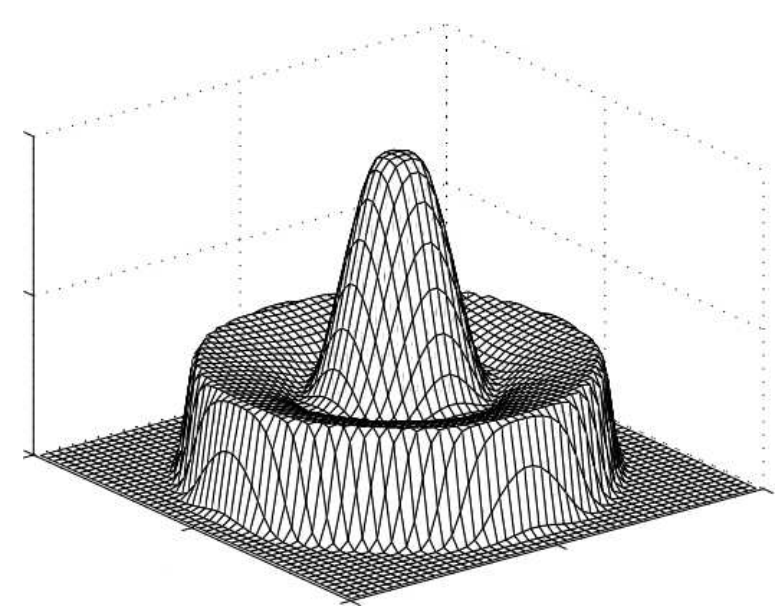

(b) Depois do colapso

Figura 7.1: Ilustração do problema circular dam-break flow.

Para a simulação desse problema, considera-se um domínio $[-2.5,2.5] \times[-2.5,2.5], \theta=0.9$ e uma malha de $125 \times 125$ células computacionais. Inicialmente, a barreira cilíndrica tem raio $r=1$, profundidade de água dentro da barreira igual a 2 e fora da barreira igual a 1 . As condições de contorno consideradas são extrapolação de ordem zero nas direções $x$ e $y$. De acordo com LeVeque [43] e Toro [71], uma boa aproximação para a solução exata desse problema (considerada como solução de referência na Figura 7.4) é obtida a partir da resolução da equação 1D dada por

$$
\left(\begin{array}{c}
h \\
h U
\end{array}\right)_{t}+\left(\begin{array}{c}
h U \\
h U^{2}+\frac{1}{2} g h^{2}
\end{array}\right)_{r}=\left(\begin{array}{c}
-\frac{h U}{r} \\
-\frac{h U^{2}}{r}
\end{array}\right)
$$

onde $U=U(x, t)$ é a velocidade radial. Esta foi resolvida no CLAWPACK pelo método de Godunov de primeira ordem com termo de correção, empregando-se o limitador de fluxo MC com malha de 2000 células computacionais e $\theta=0.9$.

As Figuras 7.2 e 7.3 mostram os resultados numéricos obtidos em $t=1.5$ para o contorno da profundidade $h$ no plano $x \perp y$ e a elevação da superfície de água em 3D para os esquemas PFDPUS, ADBQUICKEST, TOPUS e SDPUS-C1. É possível notar a partir das figuras que mostram o contorno da profundidade $h$ no plano $x \perp y$ que todos os esquemas estudados preservam bem a simetria circular, como esperado, sem o aparecimento de ondas irregulares. Além disso, as figuras que ilustram a elevação da superfície de água em 3D mostram que há uma onda de choque circular propagando-se para fora e uma onda de rarefação circular para 
dentro como foi dito na descrição do problema. Como pode-se notar, em geral, os resultados apresentam um comportamento com simetria e concordam muito bem com aqueles encontrados nas referências [43] e [71].
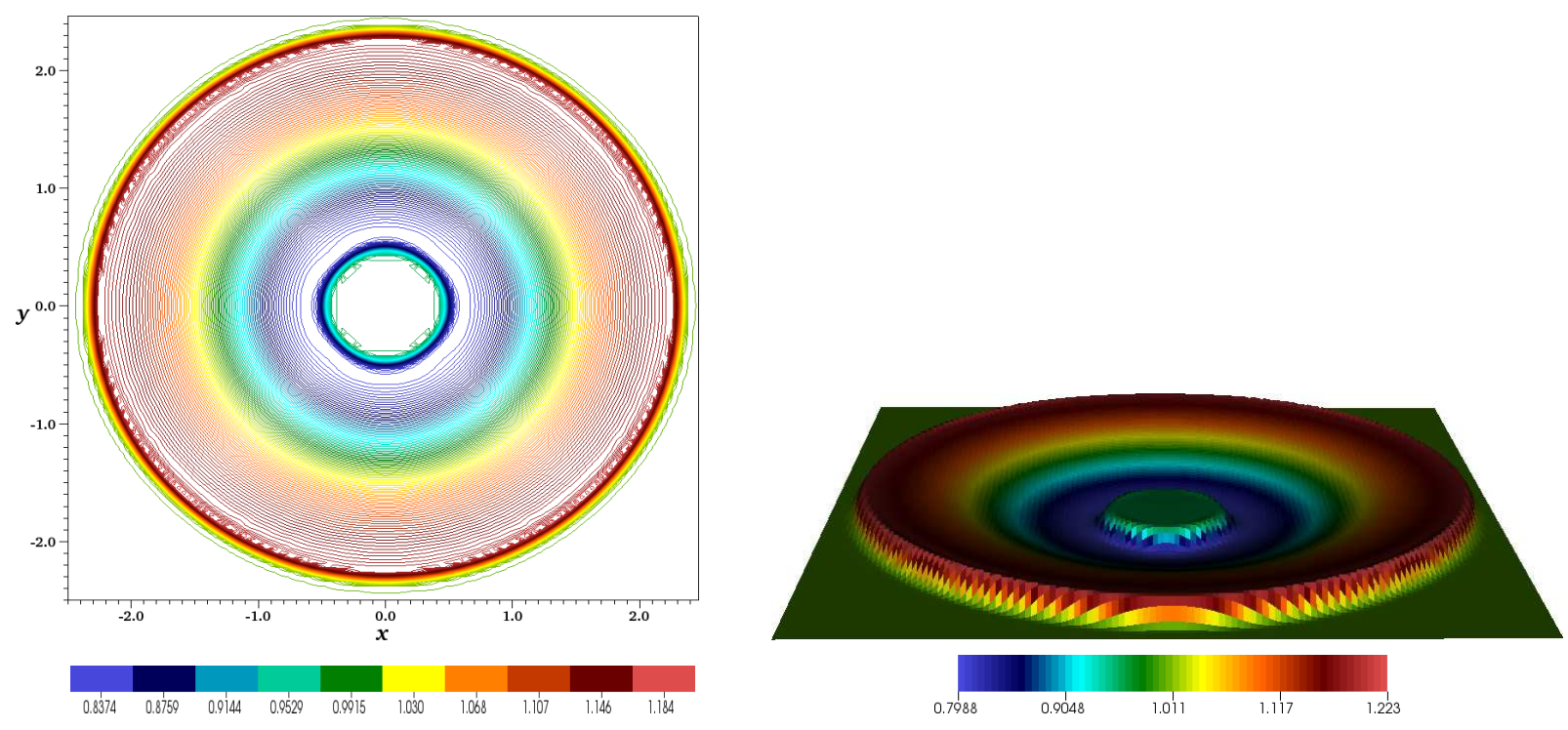

(a) PFDPUS
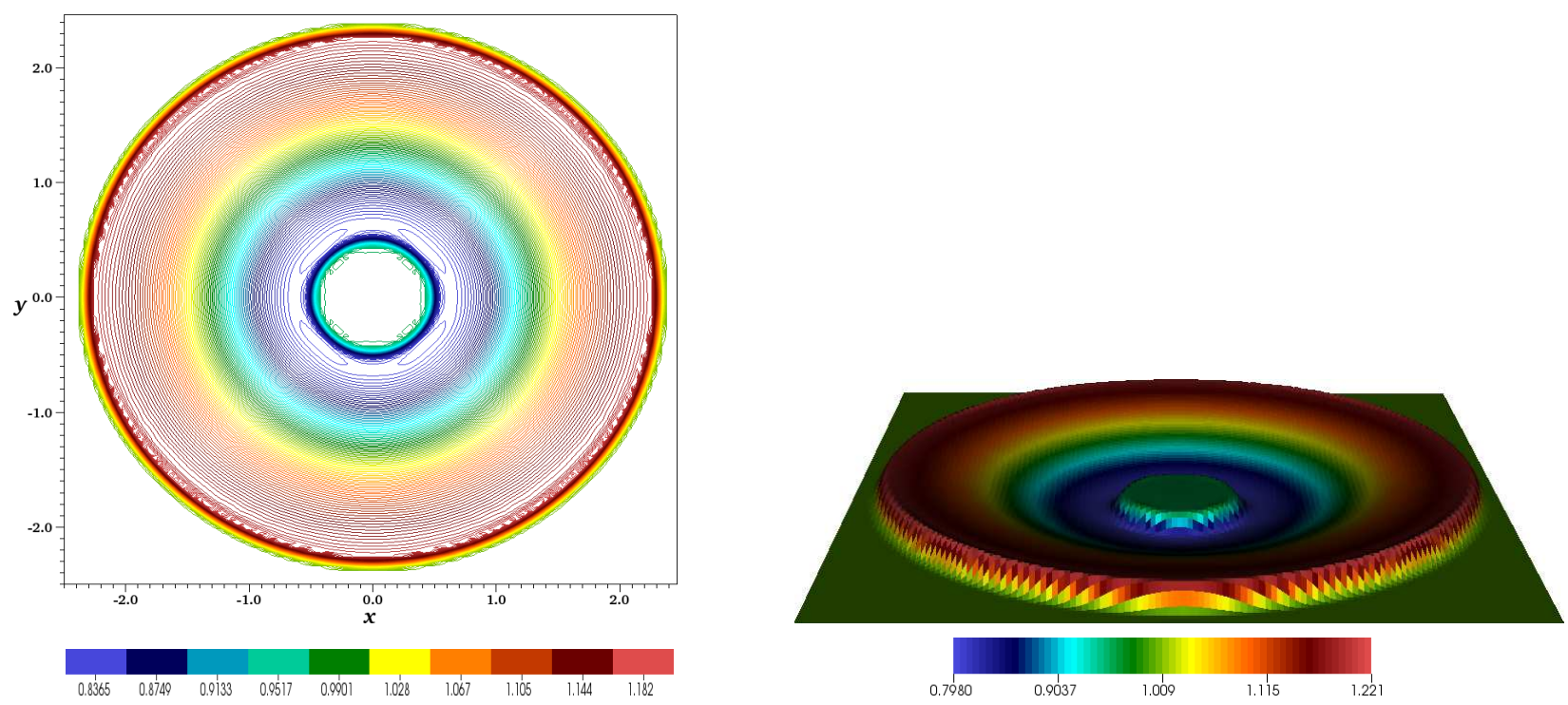

(b) ADBQUICKEST

Figura 7.2: Resultados numéricos para o problema circular dam-break flow com respeito ao contorno da profundidade $h$ no plano $x \perp y$ e a elevação da superfície de água em 3D em $t=1.5$ usando uma malha de $125 \times 125$ e $\theta=0.9$.

Para complementar a análise, a Figura 7.4 apresenta a comparação entre a solução de referência dada por (7.1) e os resultados gerados pelos esquemas PFDPUS, ADBQUICKEST, TOPUS e SDPUS-C1 considerando a variação da profundidade $h$ em função da distância ao longo do corte $y=0(x \in[0,2.5])$. Pode-se notar através das ampliações que, próximo a onda 

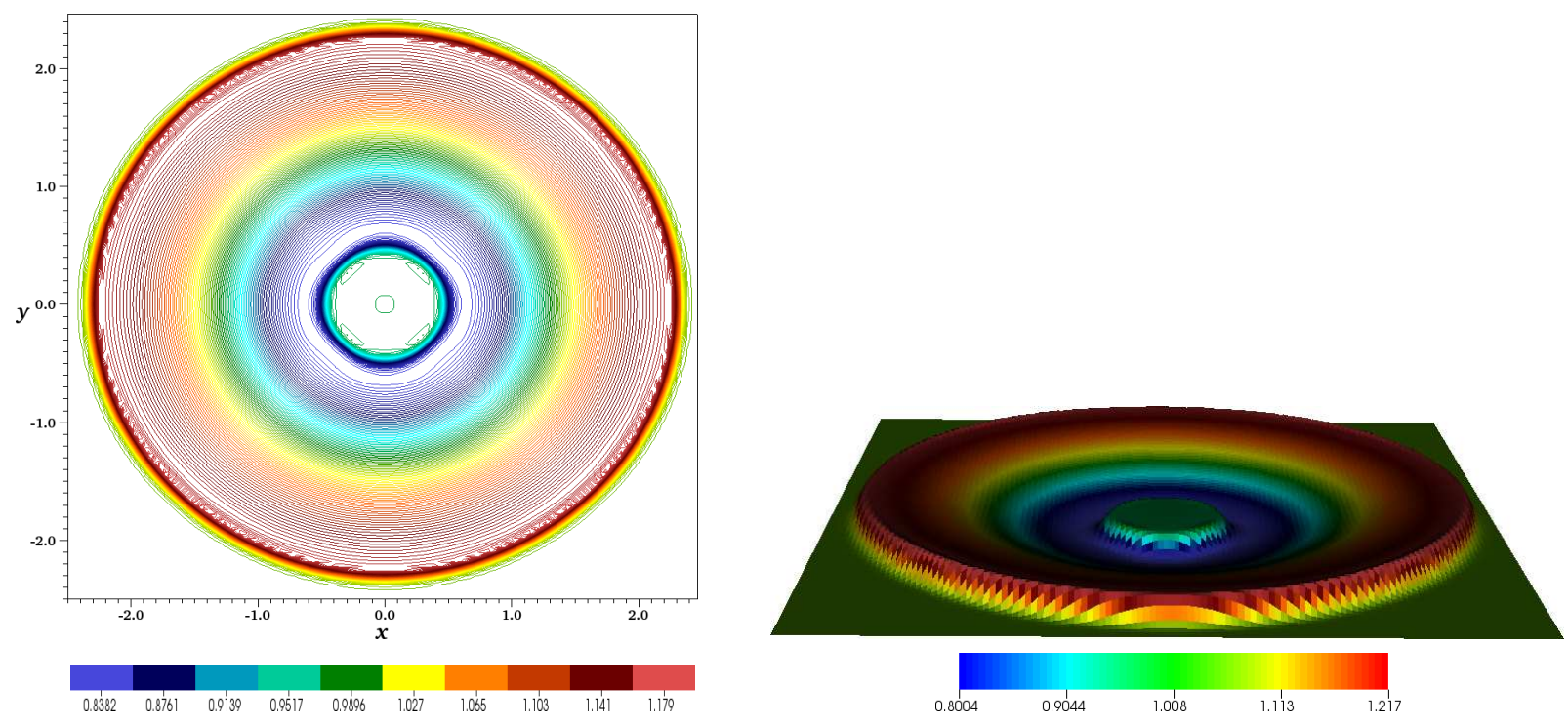

(c) TOPUS
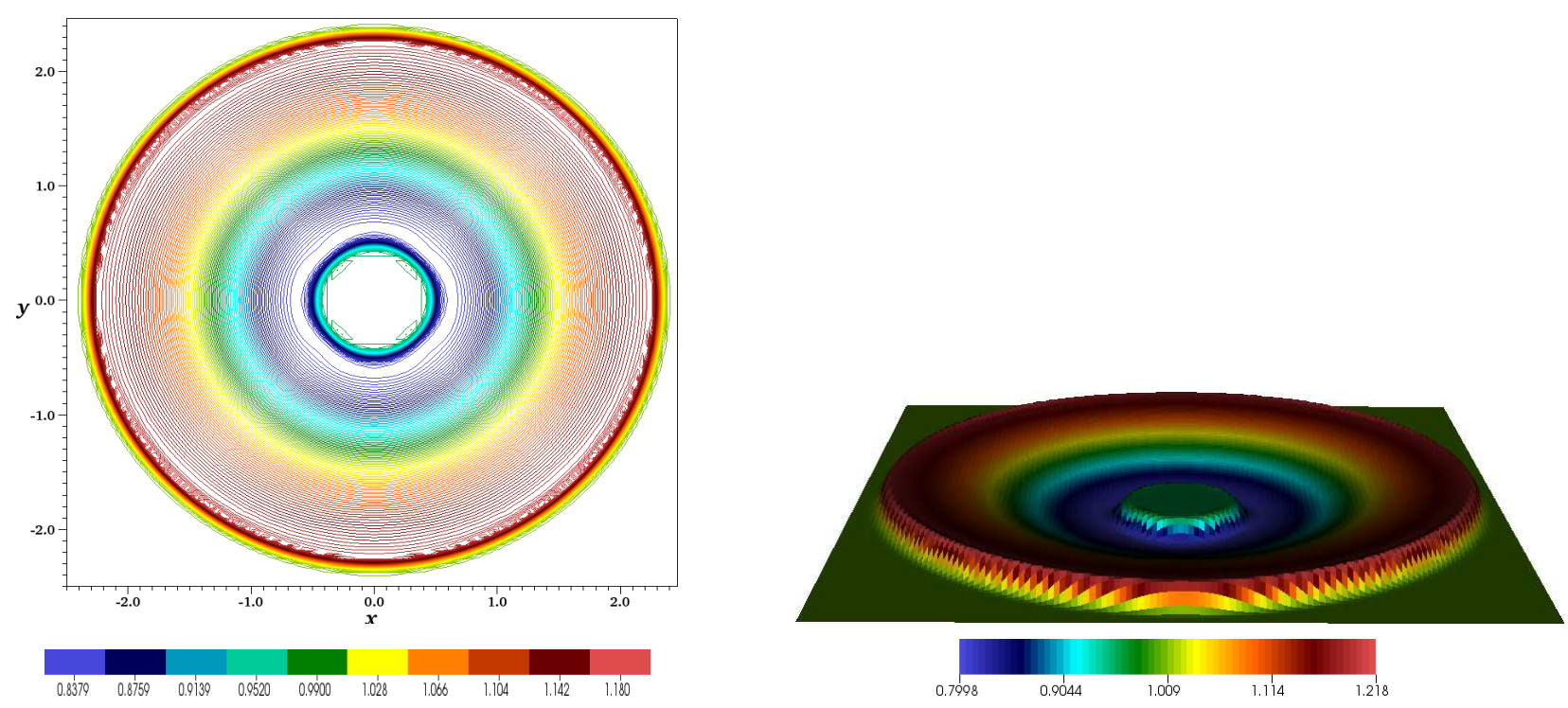

(d) SDPUS-C1

Figura 7.3: Resultados numéricos para o problema circular dam-break flow com respeito ao contorno da profundidade $h$ no plano $x \perp y$ e a elevação da superfície de água em 3D em $t=1.5$ usando uma malha de $125 \times 125$ e $\theta=0.9$.

de choque, todos os esquemas apresentam comportamentos semelhantes. No entanto, vale salientar, que na onda de rarefação o esquema PFDPUS apresenta ligeiramente os melhores resultados, sendo que o pior comportamento é obtido pelo esquema ADBQUICKEST (mais dissipativo). 


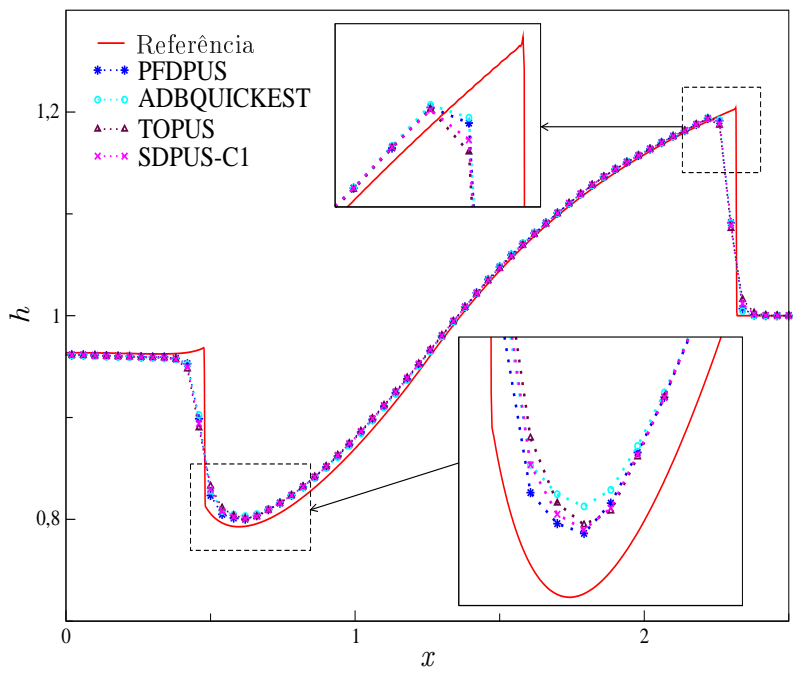

Figura 7.4: Comparação dos resultados numéricos, em $t=1.5$, para a variação da profundidade $h$ em função da distância ao longo de $y=0$ para o problema circular dam-break flow.

\subsubsection{Equações de Euler}

O sistema de equações de Euler é formulado pela lei de conservação 2D (2.21), possuindo vetor de variáveis conservadas (2.27) e funções fluxo (2.28) e (2.29). Aqui, apresentam-se resultados numéricos para dois problemas modelados por essas equações, denominados quatro ondas de choque e two non-neighbouring slip lines.

\section{Problema 2: (Quatro ondas de choque)}

Este teste tem por objetivo investigar o desempenho de esquemas numéricos na resolução de descontinuidades. Os dados consistem em quatro valores constantes em quatro quadrantes de modo que cada par de dados gera uma única onda de choque na solução (ver detalhes em [42] e [59]). Com o passar do tempo, a interação entre as ondas levam à estruturas de ondas mais complexas. Os dados iniciais são definidos em um quadrado $[0,1] \times[0,1]$ da forma

$$
\left[\rho_{0}, u_{0}, v_{0}, P_{0}\right]^{T}= \begin{cases}{[1.5,0,0,1.5]^{T},} & \text { se } x \geq 0.8 \text { e } y \geq 0.8, \\ {[0.5323,1.206,0,0.3]^{T},} & \text { se } x<0.8 \text { e } y \geq 0.8, \\ {[0.138,1.206,1.206,0.029]^{T},} & \text { se } x<0.8 \text { e } y<0.8 \\ {[0.5323,0,1.206,0.3]^{T},} & \text { se } x \geq 0.8 \text { e } y<0.8\end{cases}
$$

e as condições de contorno utilizadas são as de extrapolação de ordem zero em ambas direções $x$ e $y$. Os resultados numéricos são calculados no pacote computacional CLAWPACK por meio do método de Godunov de primeira ordem, com termo de correção, onde os limitadores de fluxo dos esquemas PFDPUS, ADBQUICKEST, TOPUS e SDPUS-C1 são acrescentados. Para simulação, malha de $1000 \times 1000$ células computacionais e $\theta=0.8$ são considerados.

Nas Figuras 7.5 e 7.6, são apresentados os resultados numéricos obtidos em $t=0.8$ com 
respeito aos contornos da densidade e pressão, respectivamente. Primeiramente, pode-se notar, que todos os esquemas estão de acordo com a estrutura básica da solução na região onde os quatro choques interagem, mostrando boa concordância com os resultados encontrados em Ricchiuto et al. [55], LeVeque [42] e Cada e Torrilhon [8]. Além disso, tanto para o contorno da densidade quanto para contorno da pressão, todos os esquemas apresentam uma nítida resolução dos quatro choques que separam as quatro regiões de estados constantes. No entanto, pode-se observar claramente que o esquema PFDPUS apresenta resultados com mais detalhes para o contorno da densidade (ver Figura 7.5-(a)) e pressão (ver Figura 7.6-(a)), com o aparecimento de pequenos vórtices na região das linhas de contato deslizantes (região central e topo direito). Os esquemas TOPUS e SDPUS-C1 apresentam resultados mais compressíveis (com suavização).

\section{Problema 3: (Two non-neighbouring slip lines)}

Os dados iniciais para este teste consistem, como no Problema 2, em quatro valores constantes em quatro quadrantes de modo que cada par de dados gera somente um tipo de onda na solução. A diferença é que os dados geram duas linhas deslizantes (entre os quadrantes 1 e 2 e os quadrantes 3 e 4), uma onda de choque (entre os quadrantes 3 e 2) e uma onda de rarefação (entre os quadrantes 4 e 1). As duas linhas deslizantes, que dividem a solução em duas partes, juntam-se em um vórtice dentro da área subsônica. Um choque viaja do quadrante 3 para o quadrante 2 e termina na linha deslizante situada entre os quadrantes 3 e 4 . Abaixo da onda de rarefação, que se move do quadrante 1 para o quadrante 4, a área subsônica é parcialmente delimitada por uma onda de choque (ver detalhes em [59] e [58]). Em geral, a estrutura da solução é caracterizada por um vórtice que gira no sentido horário. Resumidamente, os dados inicias são definidos em $[0,1] \times[0,1]$ da forma

$$
\left[\rho_{0}, u_{0}, v_{0}, P_{0}\right]^{T}=\left\{\begin{array}{lll}
{[1,0,-0.4,1]^{T},} & \text { se } x \geq 0.5 \text { e } y \geq 0.5 \\
{[2,0,-0.3,2]^{T},} & \text { se } x<0.5 \text { e } y \geq 0.5 \\
{[1.0625,0,0.2145,0.4]^{T},} & \text { se } x<0.5 \text { e } y<0.5 \\
{[0.5197,0,-1.1259,0.4]^{T},} & \text { se } x \geq 0.5 \text { e } y<0.5
\end{array}\right.
$$

e as condições de contorno são de extrapolação de ordem zero em ambas direções $x$ e $y$. Novamente, os resultados numéricos são calculados com o auxílio do pacote computacional CLAWPACK através do método de Godunov de primeira ordem com termo de correção onde os esquemas PFDPUS, ADBQUICKEST, TOPUS e SDPUS-C1 são implementados. Para simulação, malha de $400 \times 400$ células computacionais e $\theta=0.8$ são considerados.

Os resultados das simulações numéricas para contorno da densidade, em $t=0.3$, são apresentados na Figura 7.7. Em geral, os esquemas capturam bem as linhas deslizantes, a onda de choque e de rarefação, mostrando com nitidez a formação do vórtice. Além disso, todos os esquemas fornecem resultados comparáveis com os encontrados em [59] e [58]. 


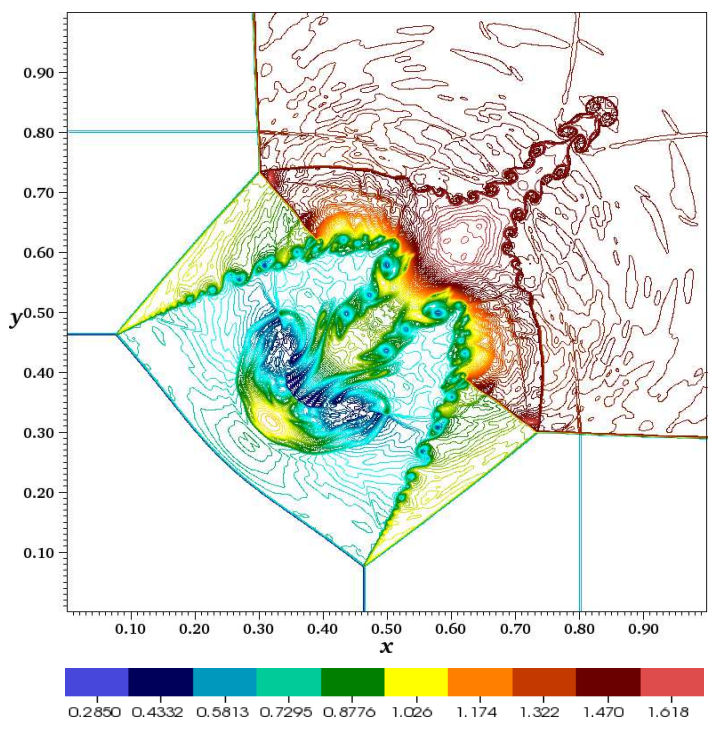

(a) PFDPUS

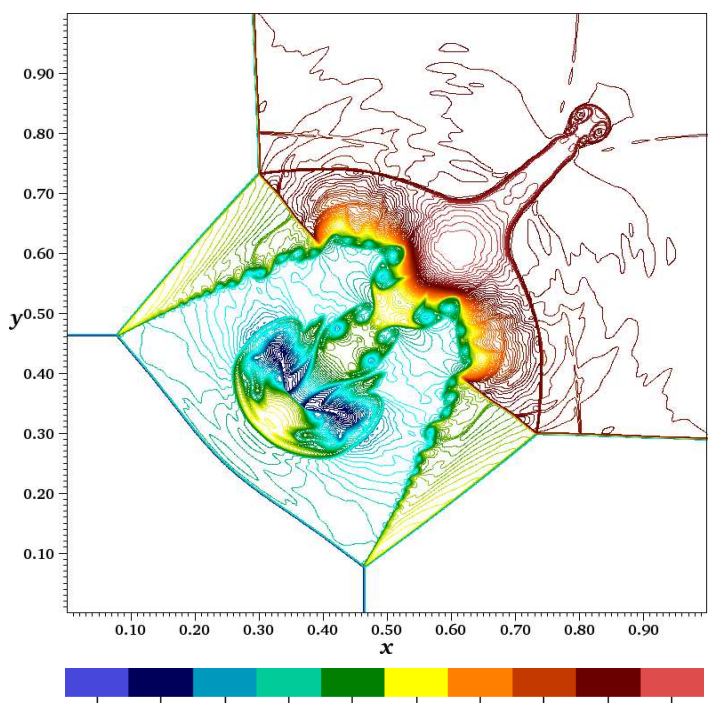

(c) TOPUS

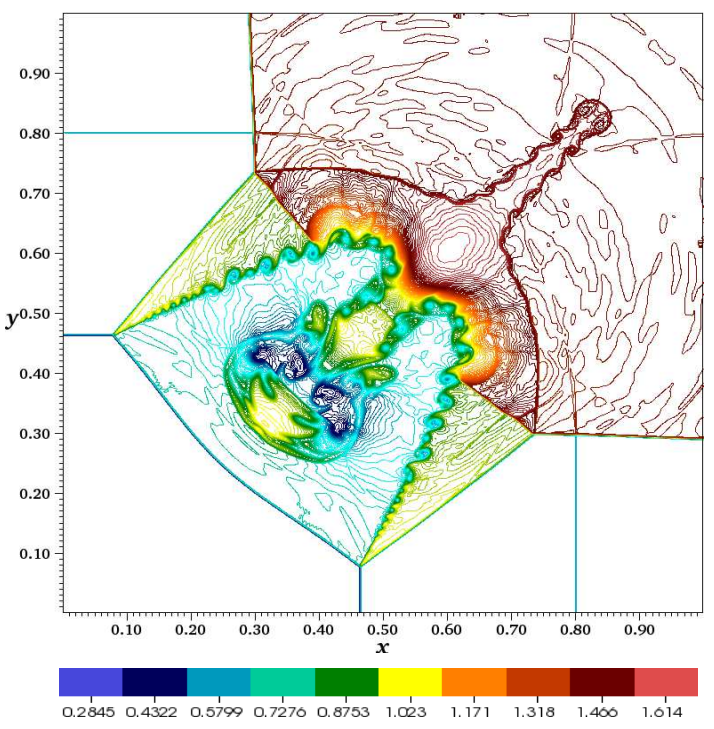

(b) ADBQUICKEST

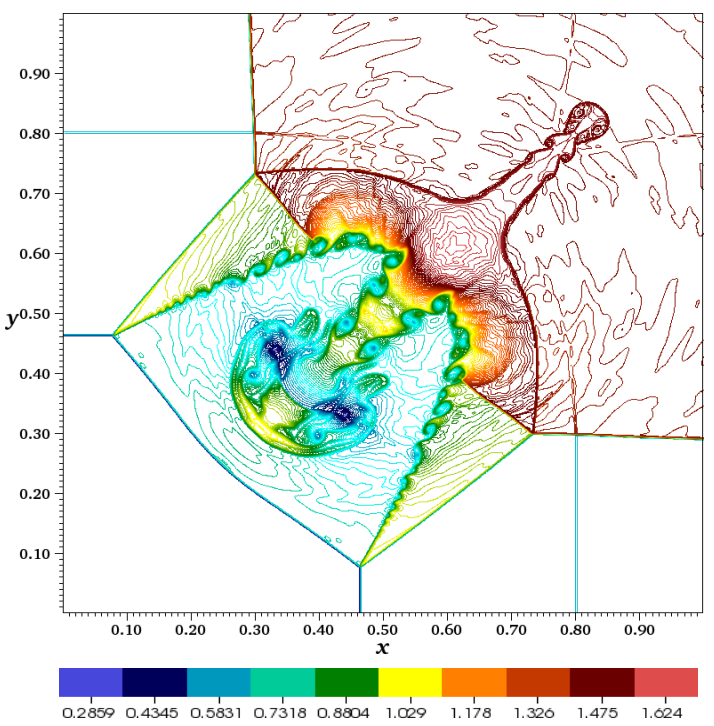

(d) SDPUS-C1

Figura 7.5: Resultados numéricos para o problema das quatro ondas de choque em $t=0.8$ com respeito ao contorno da densidade usando $1000 \times 1000$ células computacionais e $\theta=0.8$.

\subsubsection{Equações MHD}

Esse sistema de equações MHD é formulado pela lei de conservação 2D (2.21), adotando-se vetor de variáveis conservadas (2.32) e funções fluxo (2.33) e (2.34). Um dos vários problemas modelados por esse sistema de equações e estudado nesse trabalho é o problema de Orszag-Tang.

\section{Problema 4: (Problema de Orszag-Tang)}

Este problema modela a evolução de um sistema de vórtices compressíveis que envolve interações entre várias ondas de choque viajando com diferentes velocidades, o que o torna bastante atrativo do ponto de vista numérico (ver Balbás et al. [3] e Jiang e Wu [31]). No 


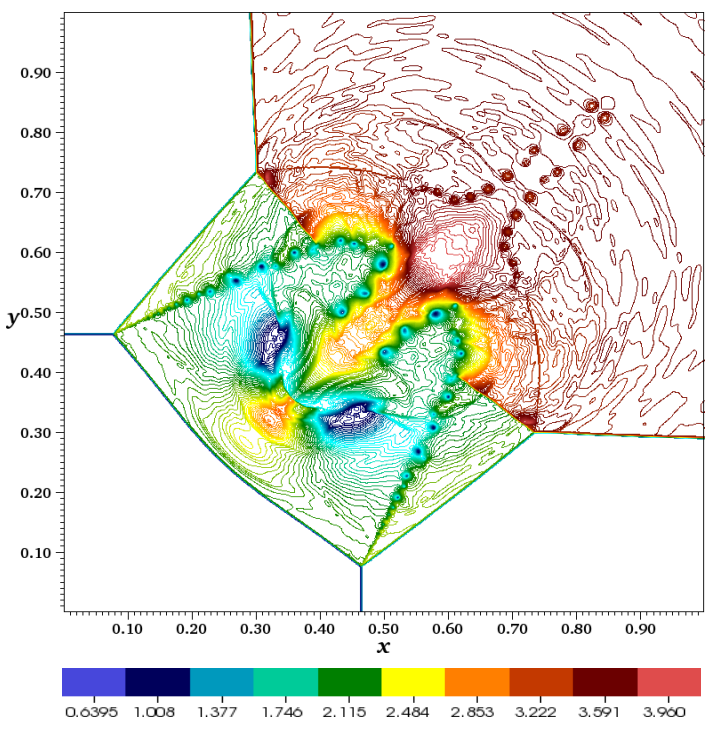

(a) PFDPUS

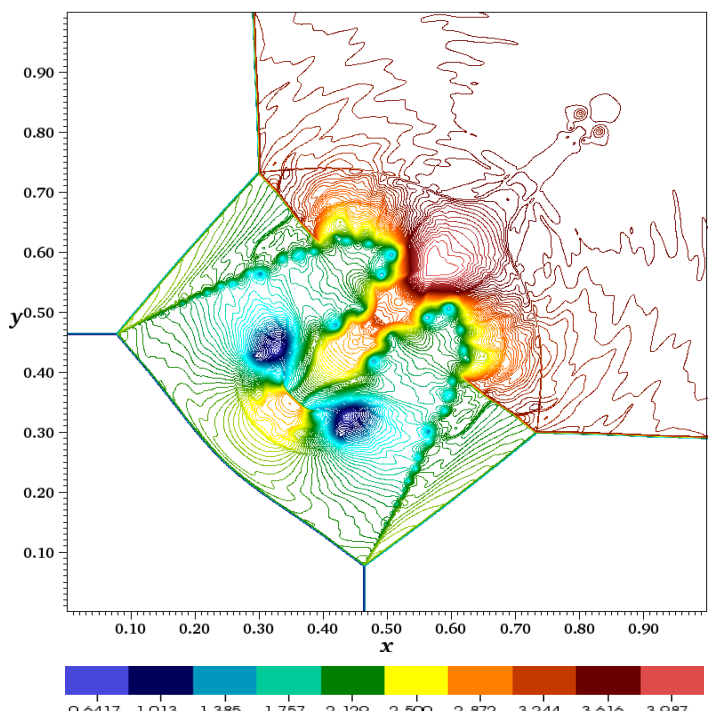

(c) TOPUS

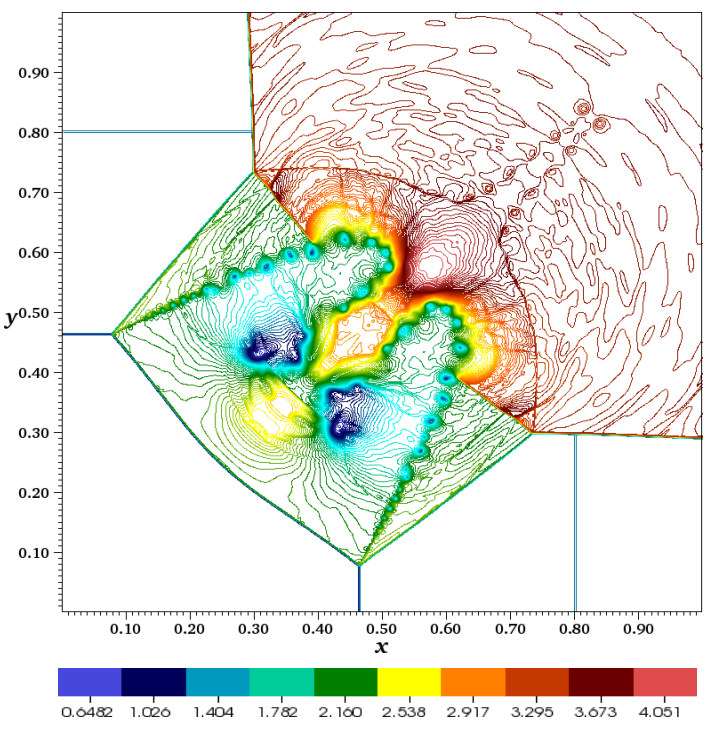

(b) ADBQUICKEST

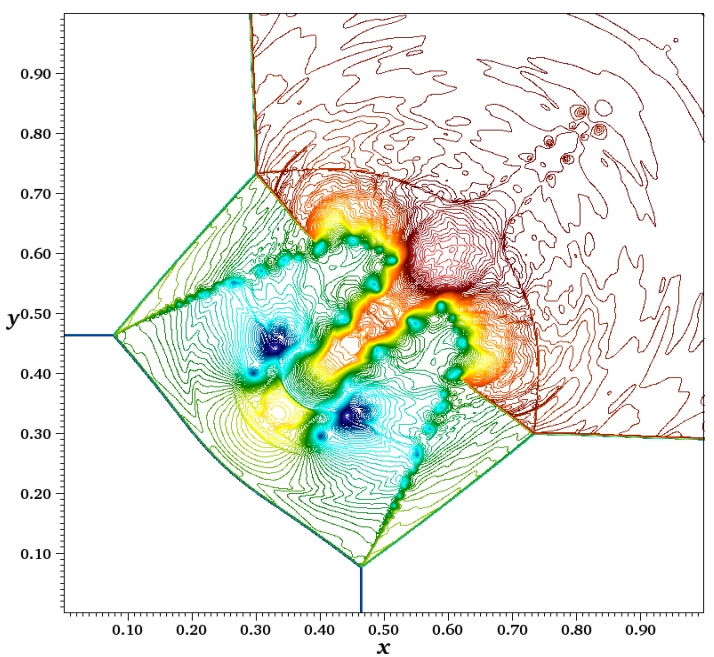

$\begin{array}{llllllllll}0.0450 & 1.020 & 1.394 & 1.708 & 2.142 & 2.510 & 2.890 & 3.264 & 3.038 & 4.013\end{array}$

(d) SDPUS-C1

Figura 7.6: Resultados numéricos para o problema das quatro ondas de choque em $t=0.8 \mathrm{com}$ respeito ao contorno da pressão usando $1000 \times 1000$ células computacionais e $\theta=0.8$.

início os dados são suaves, mas gradualmente o fluxo se torna mais complexo a partir da transição para a turbulência.

O vórtice inicial é dado por

$$
\begin{array}{ccc}
\rho=\gamma^{2} & u=-\operatorname{sen}(y) & B_{1}=-\operatorname{sen}(y), \\
P=\gamma & v=\operatorname{sen}(x) & B_{2}=\operatorname{sen}(y), \\
w=0 & B_{3}=0 .
\end{array}
$$

O domínio computacional considerado é $[0,2 \pi] \times[0,2 \pi]$ e as condições de contorno são periódicas 


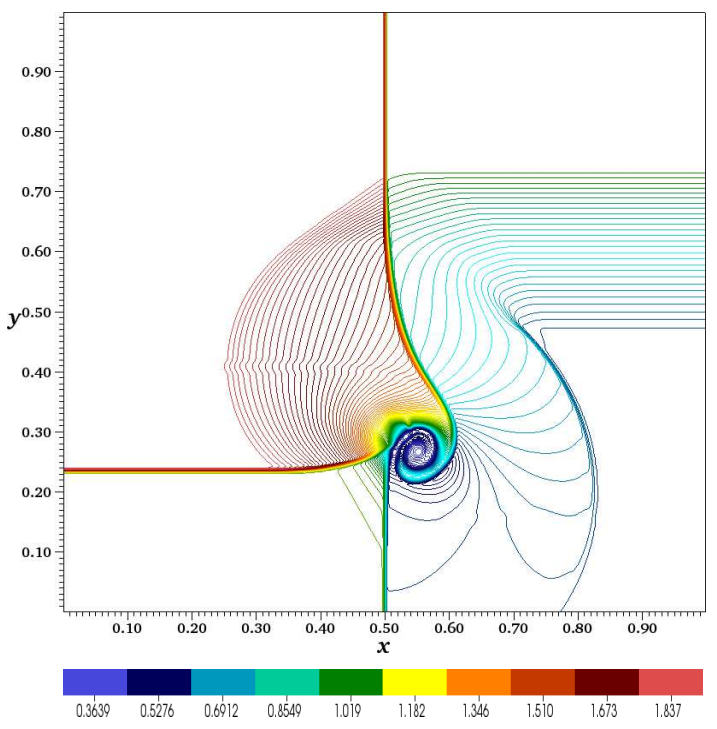

(a) PFDPUS

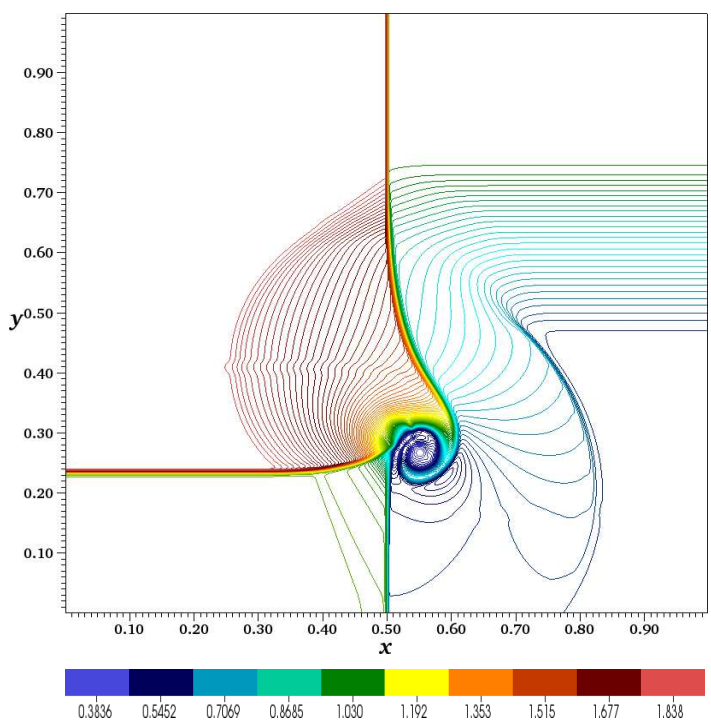

(c) TOPUS

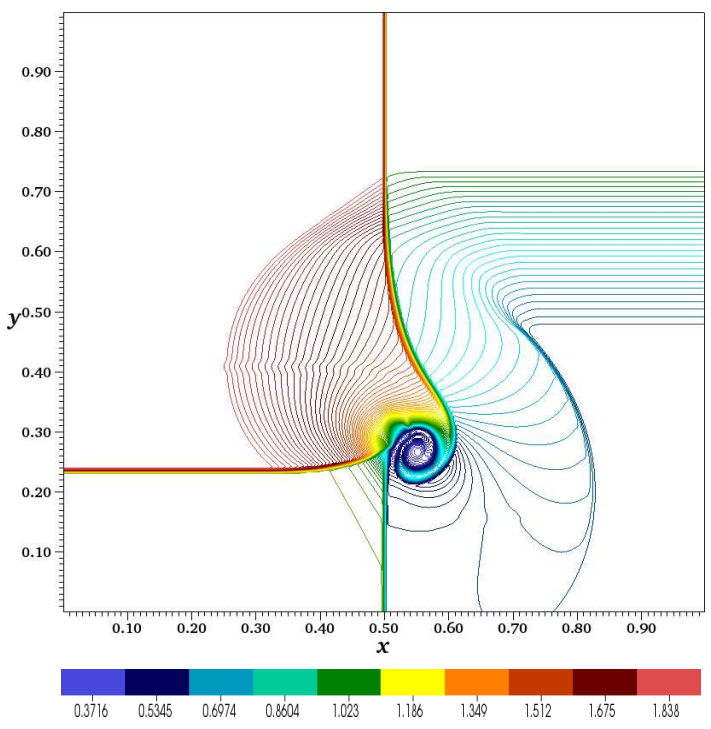

(b) ADBQUICKEST

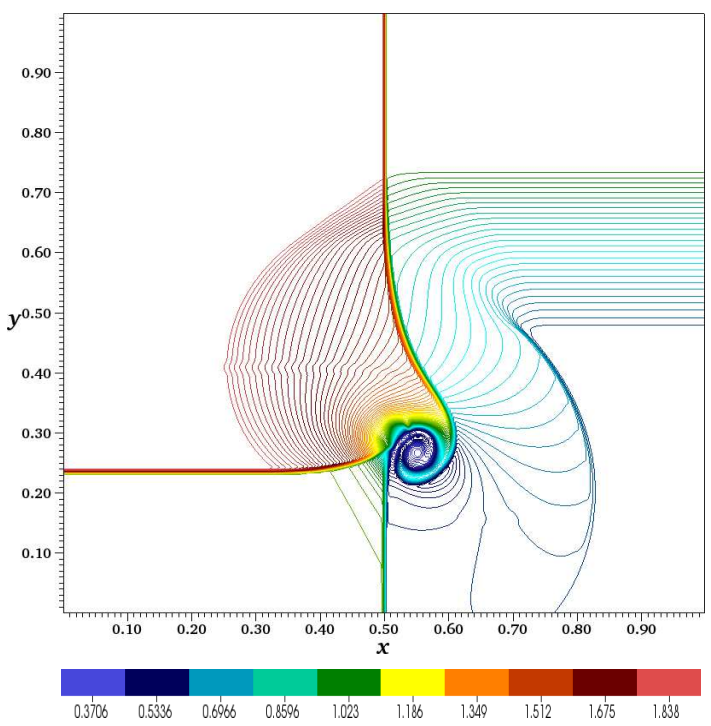

(d) SDPUS-C1

Figura 7.7: Resultados numéricos para o problema two non-neighbouring slip lines em $t=0.3$ com respeito ao contorno da densidade usando $400 \times 400$ células computacionais e $\theta=0.8$.

nas direções $x$ e $y$. As soluções numéricas são obtidas com o auxílio do CLAWPACK onde os limitadores de fluxo dos esquemas PFDPUS, ADBQUICKEST, TOPUS e SDPUS-C1 são implementados no termo de correção.

Nas Figuras 7.8 e 7.9 são apresentados, respectivamente, os resultados numéricos para os contornos da densidade e pressão, em $t=3$, considerando-se $192 \times 192$ células computacionais e $\theta=0.8$. Pode-se ver que os resultados numéricos obtidos com todos os esquemas concordam com aqueles apresentados por Jiang e Wu [31] e que a evolução do sistema de vórtices é muito complexa e muitos choques de todas as formas são formados. Além disso, os esquemas considerados não apresentam oscilações espúrias e portanto são capazes de resolver estruturas 
bastante complexas como as apresentadas pelas equações MHD 2D.

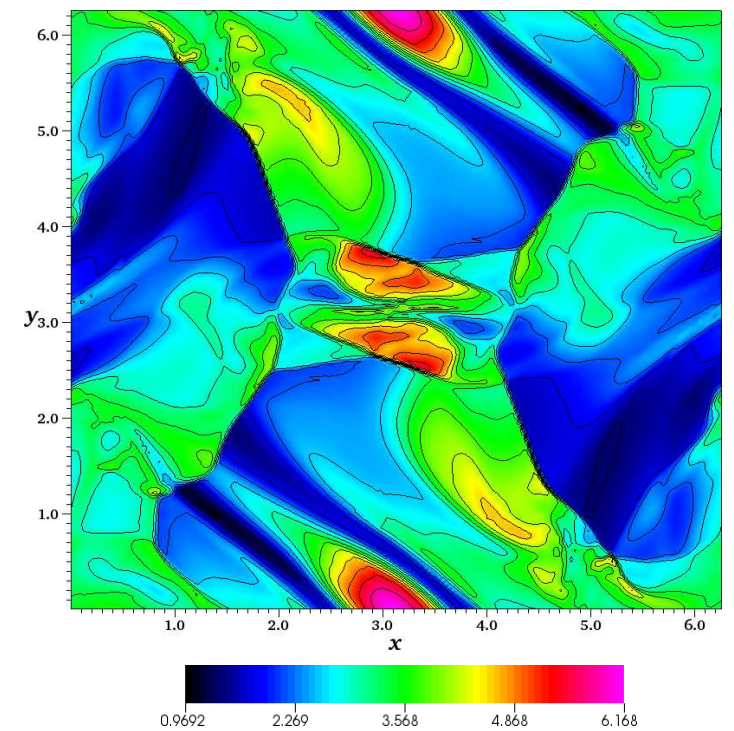

(a) PFDPUS

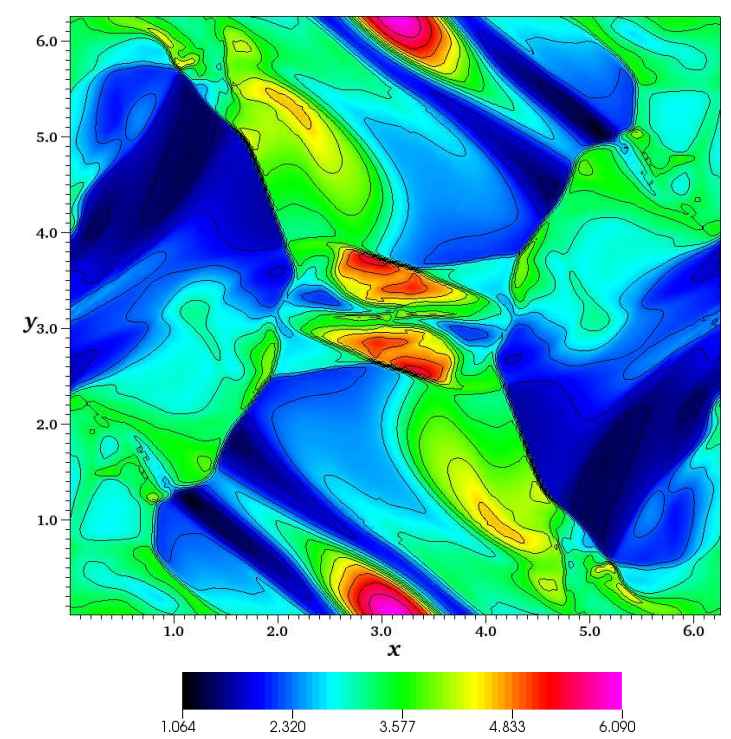

(c) TOPUS

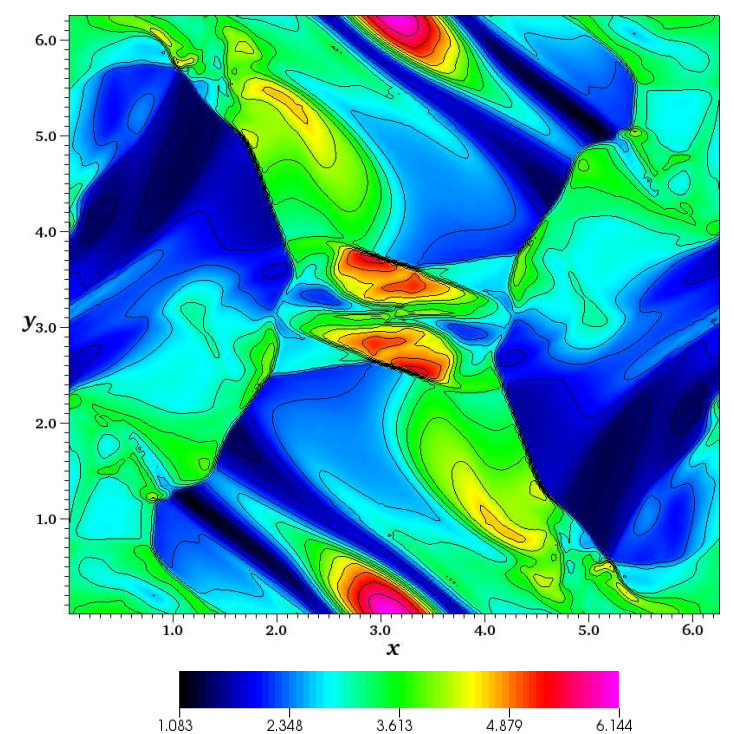

(b) ADBQUICKEST

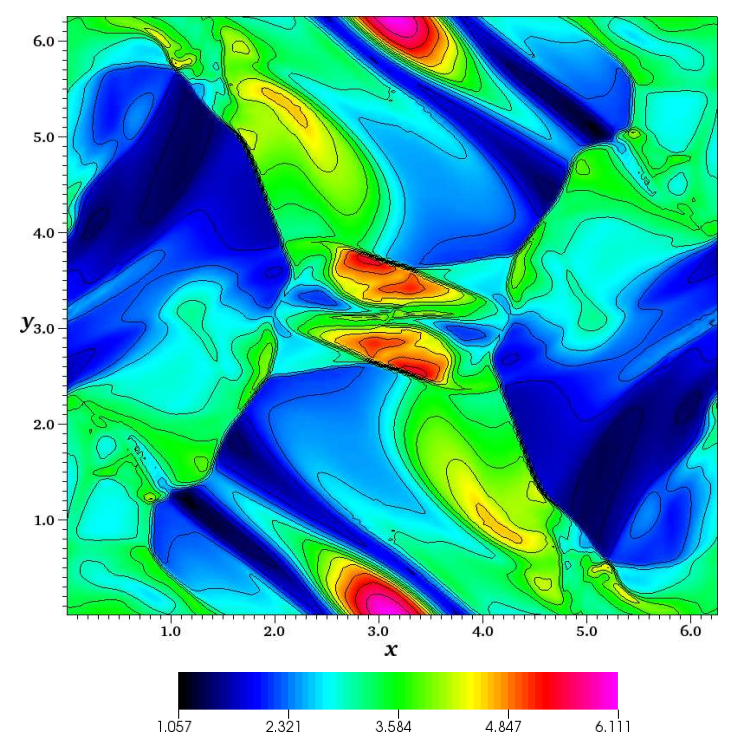

(d) SDPUS-C1

Figura 7.8: Resultados numéricos para o problema Orszag-Tang em $t=3$ com respeito ao contorno da densidade usando $192 \times 192$ células computacionais e $\theta=0.8$.

A distribuição da pressão ao longo do corte $y=0.625 \pi$ é mostrada na Figura 7.10. A solução apresentada por Balbás et al. [3] é considerada como referência para comparação dos resultados obtidos. Pode-se ver que os resultados são consistentes com a solução de referência considerada.

Para complementar a análise, a Tabela 7.1 mostra os erros e as ordens de convergência em $t=0.2$ para a densidade considerando as normas $L_{1}$ e $L_{2}$. É importante ressaltar que os erros 


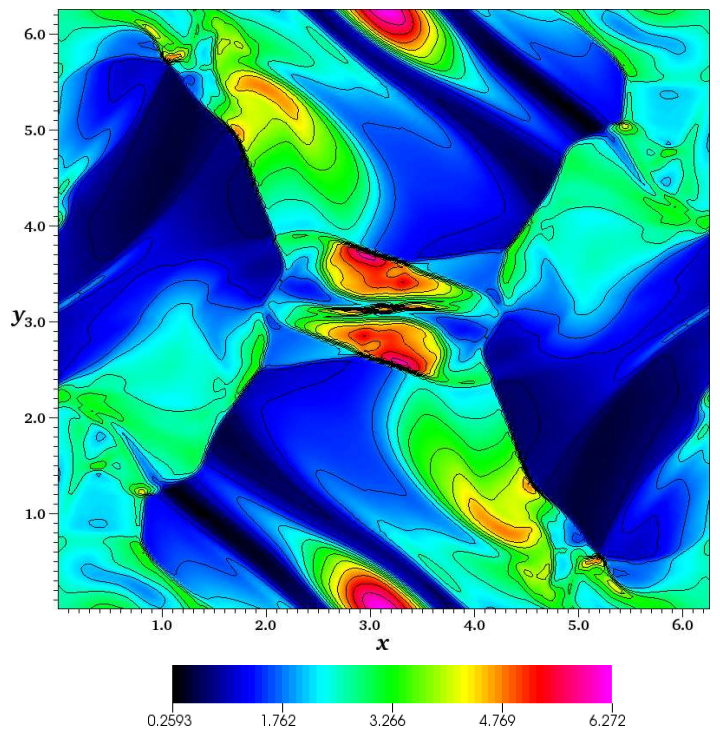

(a) PFDPUS

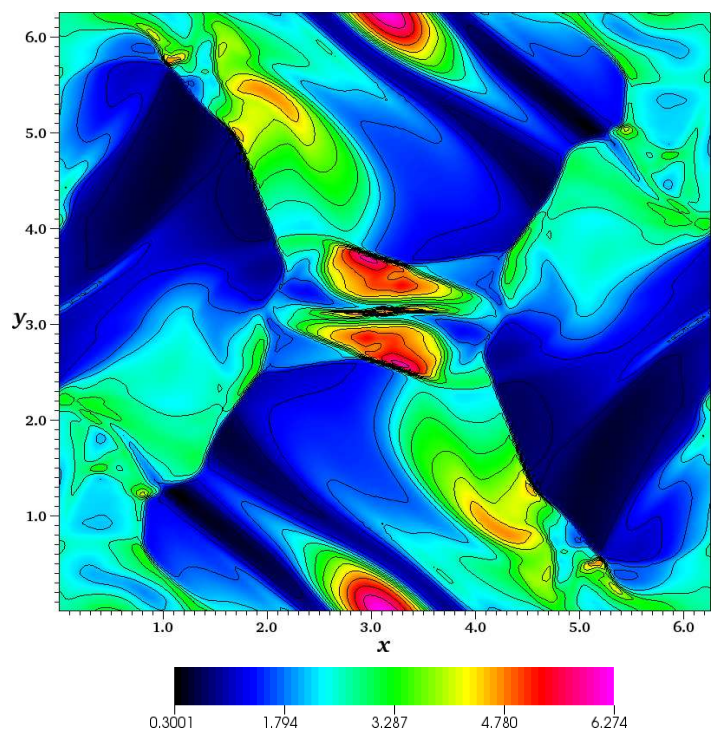

(c) TOPUS

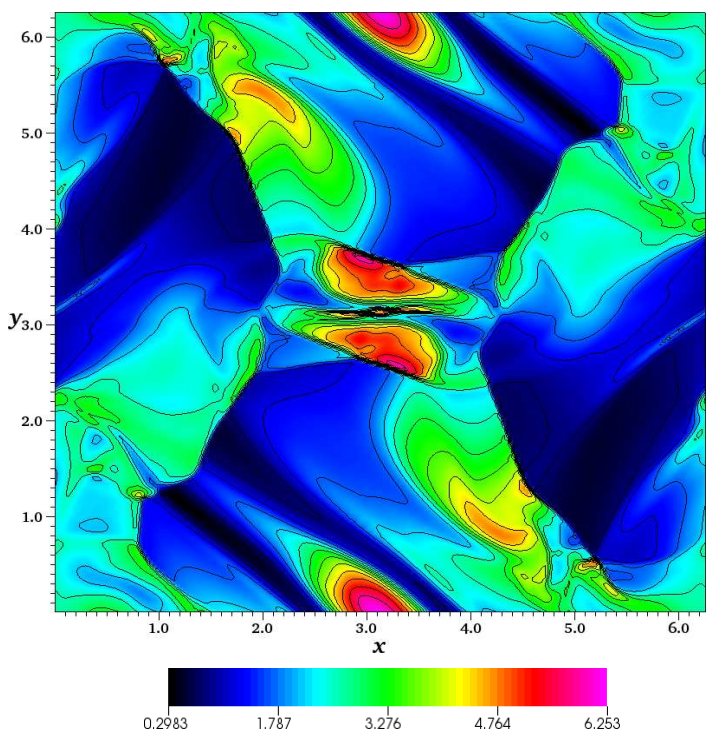

(b) ADBQUICKEST

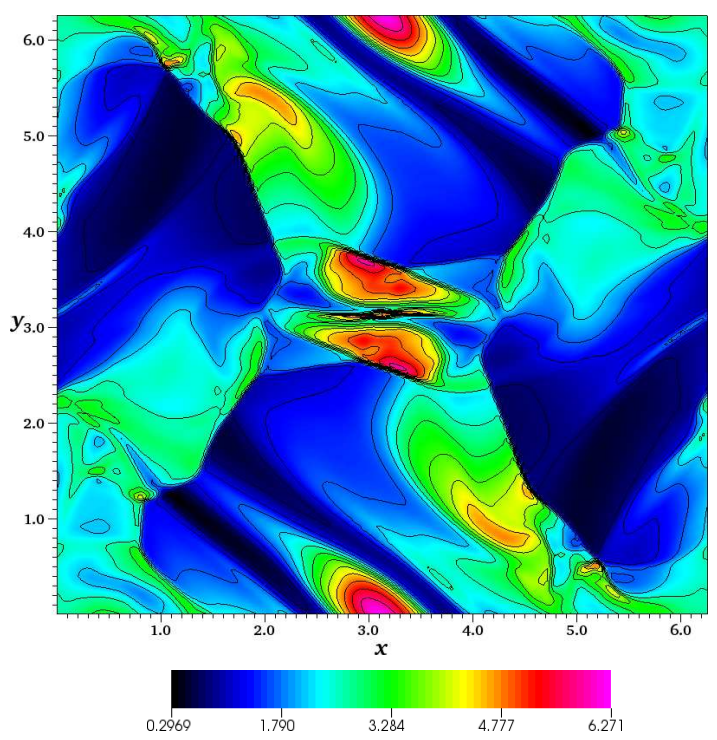

(d) SDPUS-C1

Figura 7.9: Resultados numéricos para o problema Orszag-Tang em $t=3$ com respeito ao contorno de pressão usando $192 \times 192$ células computacionais e $\theta=0.8$.

são obtidos da forma (ver Jiang e Wu [31])

$$
\left\|E_{h}\right\|=\left\|u^{h}-u^{512}\right\| h^{2}
$$

onde $u^{h}$ e $u^{512}$ são as soluções numéricas obtidas por um mesmo esquema em uma malha $N \times N$ e em uma malha $512 \times 512$ (considerada como solução de referência), respectivamente. A ordem de convergência é calculada de acordo com (2.69), cujos resultados são apresentados na Tabela 7.1. Por essa tabela, observa-se que todos os esquemas apresentam ordem de convergência similar tanto na norma $L_{1}$ quanto na norma $L_{2}$. Além disso, é possível notar que todos os esquemas 


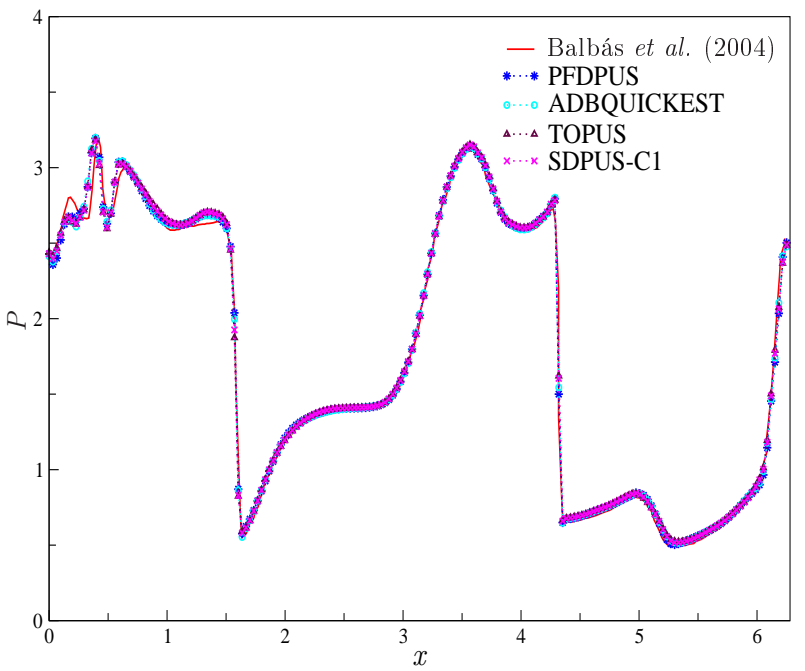

Figura 7.10: Comparação entre os resultados de Balbás et al. [3] e os numéricos obtidos para a distribuição da pressão ao longo do corte $y=0.625 \pi$ para o problema de Orszag-Tang em $t=3$.

apresentam ordem de convergência maior que dois na norma $L_{1}$ para todas as malhas, e, na norma $L_{2}$ alcançam segunda ordem somente na malha $256 \times 256$.

\subsection{Escoamentos incompressíveis laminares 2D}

Nesta seção, são apresentados resultados numéricos para problemas de escoamentos incompressíveis 2D, com superfícies livres móveis, no regime laminar. Para a simulação desses problemas, é utilizado o ambiente de simulação Freeflow 2D de Castelo et al. [9], adaptado com o esquema PFDPUS. Em particular, o seu desempenho é testado na solução do colapso de uma coluna de fluido e do jato livre sobre uma superfície rígida impermeável.

\subsubsection{Colapso de uma coluna de fluido}

Esse problema consiste em uma coluna de fluido em equilíbrio hidrostático confinado entre duas paredes rígidas que, instantaneamente, é colocada à ação da gravidade, sofrendo um colapso e deslizando na direção horizontal (ver representação esquemática na Figura 7.11). Conhecido na literatura como problema de Broken Dam, esse tipo de escoamento foi estudado experimentalmente no passado por Martin e Moyce [48], com o objetivo de investigar o espalhamento horizontal do fluido (posição frontal do fluido) e a taxa de decaimento (altura) da coluna de fluido com o tempo. Mais tarde, Koshizuka e Oka [33] repetiram esse experimento com o intuito de validar um código semi-implícito de simulação. Recentemente, Colagrossi e Landrini [11] apresentaram dados numéricos, teórico e experimental para esse problema. Desde então, esse problema vem sendo utilizado com frequência para testar métodos numéricos para escoamentos com superfícies livres móveis (ver, por exemplo, [53] e [15]).

Para a simulação computacional, a coluna de fluido é considerada um quadrado de lados 


\begin{tabular}{|c|c|c|c|c|c|}
\hline \multirow[t]{2}{*}{ Esquemas } & \multirow[t]{2}{*}{$N \times N$} & \multicolumn{2}{|c|}{$\overline{L_{1}}$} & \multicolumn{2}{|c|}{$L_{2}$} \\
\hline & & $\mid E_{h} \|_{1}$ & $p$ & $\left|E_{h}\right|_{2}$ & $p$ \\
\hline \multirow[t]{5}{*}{ PFDPUS } & $16 \times 16$ & 0.168777 & - & 0.119679 & - \\
\hline & $32 \times 32$ & 0.033510 & 2.332440 & 0.037171 & 1.686911 \\
\hline & $64 \times 64$ & 0.008014 & 2.064031 & 0.012024 & 1.628303 \\
\hline & $128 \times 128$ & 0.001641 & 2.287975 & 0.003595 & 1.741995 \\
\hline & $256 \times 256$ & 0.000268 & 2.612466 & 0.000846 & 2.087177 \\
\hline \multirow[t]{5}{*}{ ADBQUICKEST } & $16 \times 16$ & 0.168517 & - & 0.118948 & - \\
\hline & $32 \times 32$ & 0.033852 & 2.315583 & 0.037173 & 1.677983 \\
\hline & $64 \times 64$ & 0.007958 & 2.088810 & 0.011986 & 1.632937 \\
\hline & $128 \times 128$ & 0.001642 & 2.277120 & 0.003594 & 1.737687 \\
\hline & $256 \times 256$ & 0.000269 & 2.609641 & 0.000845 & 2.088428 \\
\hline \multirow[t]{5}{*}{ TOPUS } & $16 \times 16$ & 0.171168 & - & 0.122563 & - \\
\hline & $32 \times 32$ & 0.034658 & 2.304135 & 0.038706 & 1.662886 \\
\hline & $64 \times 64$ & 0.008013 & 2.112767 & 0.011942 & 1.696464 \\
\hline & $128 \times 128$ & 0.001669 & 2.263529 & 0.003629 & 1.718594 \\
\hline & $256 \times 256$ & 0.000271 & 2.624604 & 0.000849 & 2.095341 \\
\hline \multirow[t]{5}{*}{ SDPUS-C1 } & $16 \times 16$ & 0.170659 & - & 0.121556 & - \\
\hline & $32 \times 32$ & 0.034301 & 2.314782 & 0.038163 & 1.671362 \\
\hline & $64 \times 64$ & 0.007985 & 2.102908 & 0.011914 & 1.679478 \\
\hline & $128 \times 128$ & 0.001657 & 2.269027 & 0.003610 & 1.722791 \\
\hline & $256 \times 256$ & 0.000270 & 2.617620 & 0.000848 & 2.089802 \\
\hline
\end{tabular}

Tabela 7.1: Teste de convergência para o problema de Orszag-Tang em $t=0.2$.

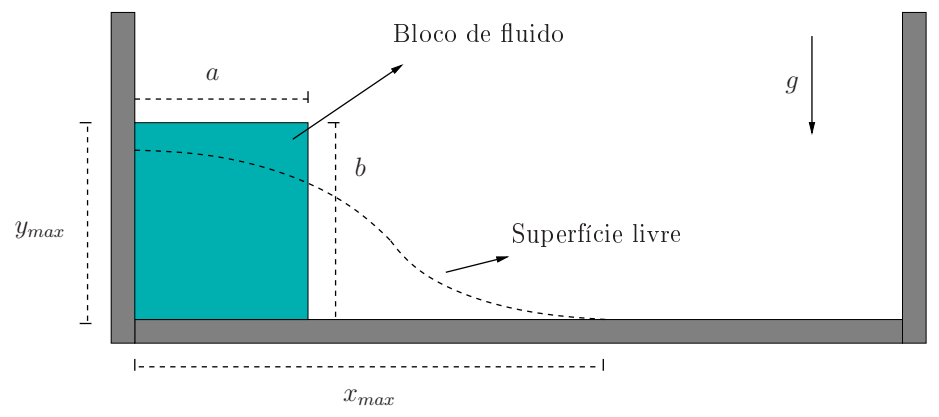

Figura 7.11: Representação esquemática do problema do colapso de uma coluna de fluido.

$a=b=0.057 m$. Para isso, o código Freeflow 2D [9], equipado com o esquema PFDPUS, é empregado nos cálculos. A condição free-splip é adotada nas paredes rígidas e os demais dados utilizados na simulação são:

- Malha: $1000 \times 200$ células computacionais $(\delta x=\delta y=0.0005 m)$;

- Dimensão do domínio: $0.5 m \times 0.1 m$;

- Escala de comprimento: $L_{0}=a=b=0.057 m$; 
- Escala de velocidade: $U_{0}=\sqrt{g L_{0}}=0.74778 \mathrm{~m} / \mathrm{s}$;

- Coeficiente de viscosidade cinemática: $\nu=10^{-6} \mathrm{~m}^{2} / \mathrm{s}$;

- Número de Reynolds: $R e=42623.27$.

Para comparar os resultados obtidos com o esquema PFDPUS consideram-se com os dados numéricos, teórico e experimental de Colagrossi e Landrini [11], para o espalhamento horizontal $\left(x_{\max }\right)$ em função do tempo. Os resultados numéricos apresentados por [11] são gerados com as formulações SPH (Smoothed Particle Hydordynamics method) de Colagrossi e Landrini [11], BEM (Bondary Element Method) de Greco et al. [25], Level Set de Colicchio et al. [12]; o dado experimental é de Martin Moyce [48] e o teórico é de Ritter [56]. Essas comparações são apresentadas na Figura 7.12. Observa-se que os resultados estão em boa concordância com as soluções encontradas em [11], especialmente quando comparados com os resultados numéricos obtidos com as formulações SPH, BEM e Level Set, o que proporciona confiabilidade no código Freeflow 2D quando adaptado com o esquema PFDPUS.

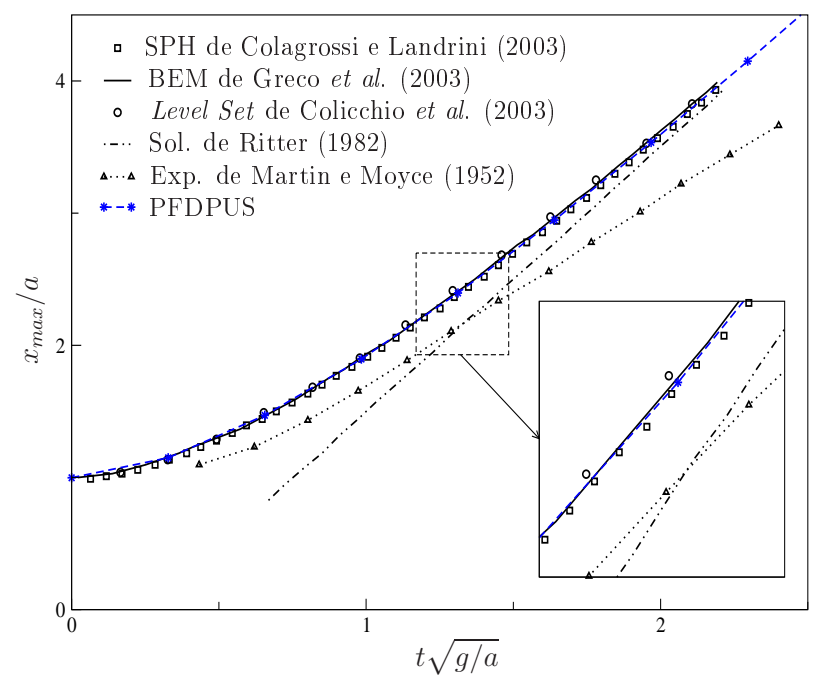

Figura 7.12: Comparação entre os dados numéricos, teórico e experimental de Colagrossi e Landrini [11] e o esquema PFDPUS para $x_{\max }$ em função do tempo obtido para o problema do colapso de fluido.

Para ilustração da evolução da superfície livre do fluido, as Figuras 7.13, 7.14 e 7.15 mostram, respectivamente, os resultados numéricos do esquema PFDPUS para os campos de pressão e velocidade (nas direções $x$ e $y$ ) nos tempos $t=0.05 s, t=0.1 s, t=0.15 \mathrm{~s}$ e $t=0.2 \mathrm{~s}$.

\subsubsection{Jato livre sobre uma superfície rígida impermeável}

O problema considerado aqui consiste no escoamento de um jato livre, em regime laminar, que incide perpendicularmente sobre uma superfície rígida impermeável sob o efeito do campo gravitacional. A geometria do problema está ilustrada na Figura 7.16. 


\section{Campo de pressão}
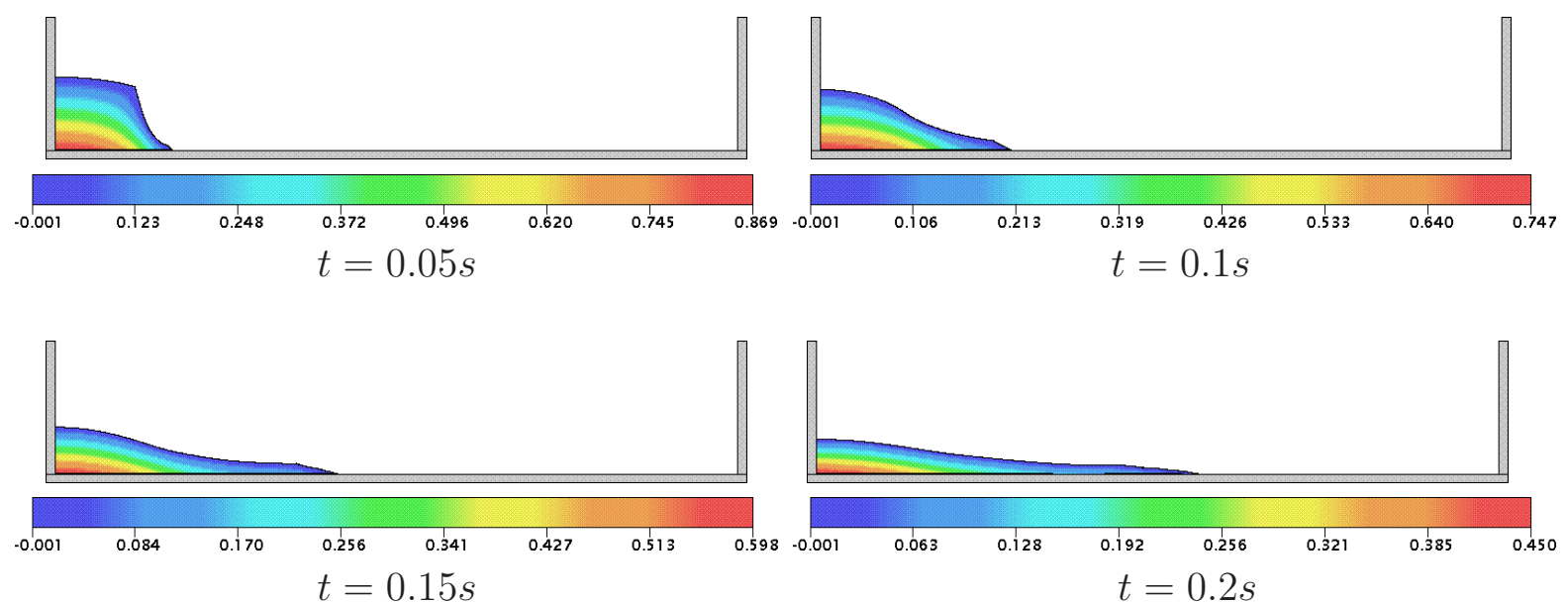

Figura 7.13: Resultados numéricos do esquema PFDPUS para a evolução da superfície livre do fluido e campo de pressão nos tempos $t=0.05 s, t=0.1 s, t=0.15 \mathrm{~s}$ e $t=0.2 \mathrm{~s}$ para o problema do colapso de fluido.

\section{Campo de velocidade na direção $x$}
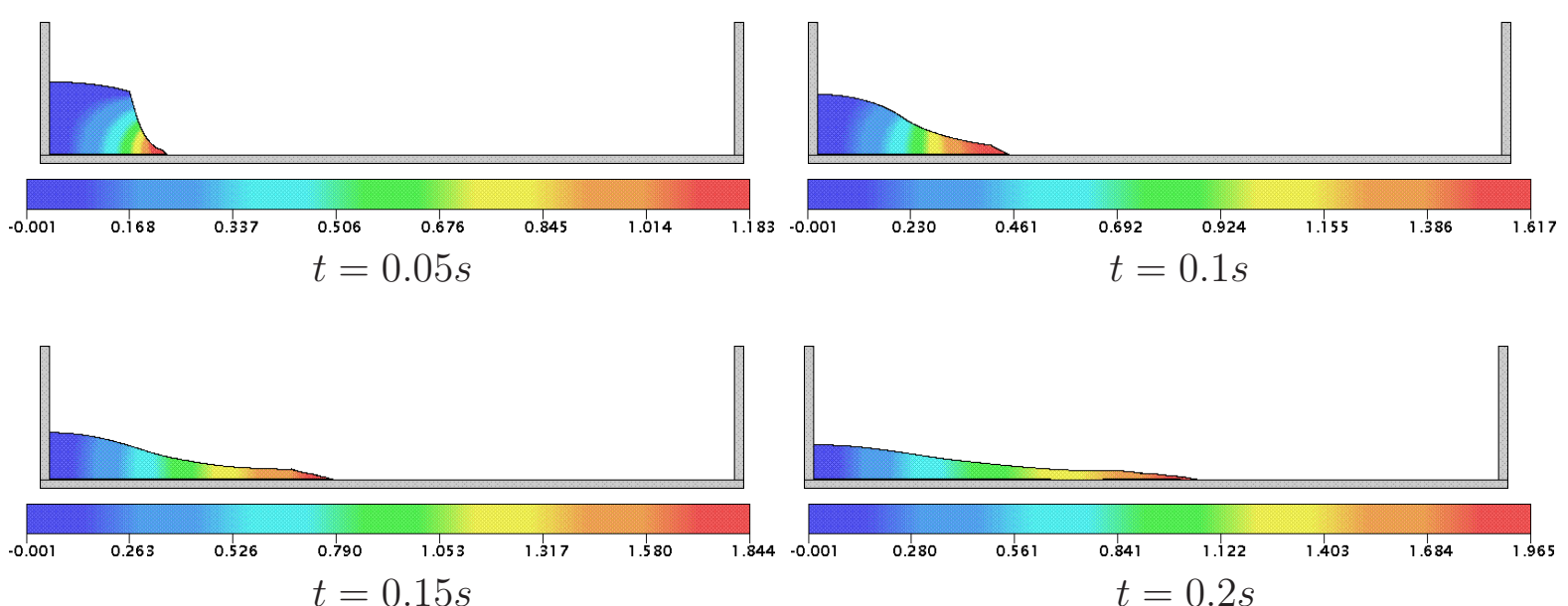

Figura 7.14: Resultados numéricos do esquema PFDPUS para a evolução da superfície livre do fluido e campo de velocidade na direção $x$ nos tempos $t=0.05 s, t=0.1 s, t=0.15 s$ e $t=0.2 s$ para o problema do colapso de fluido. 
Campo de velocidade na direção $y$
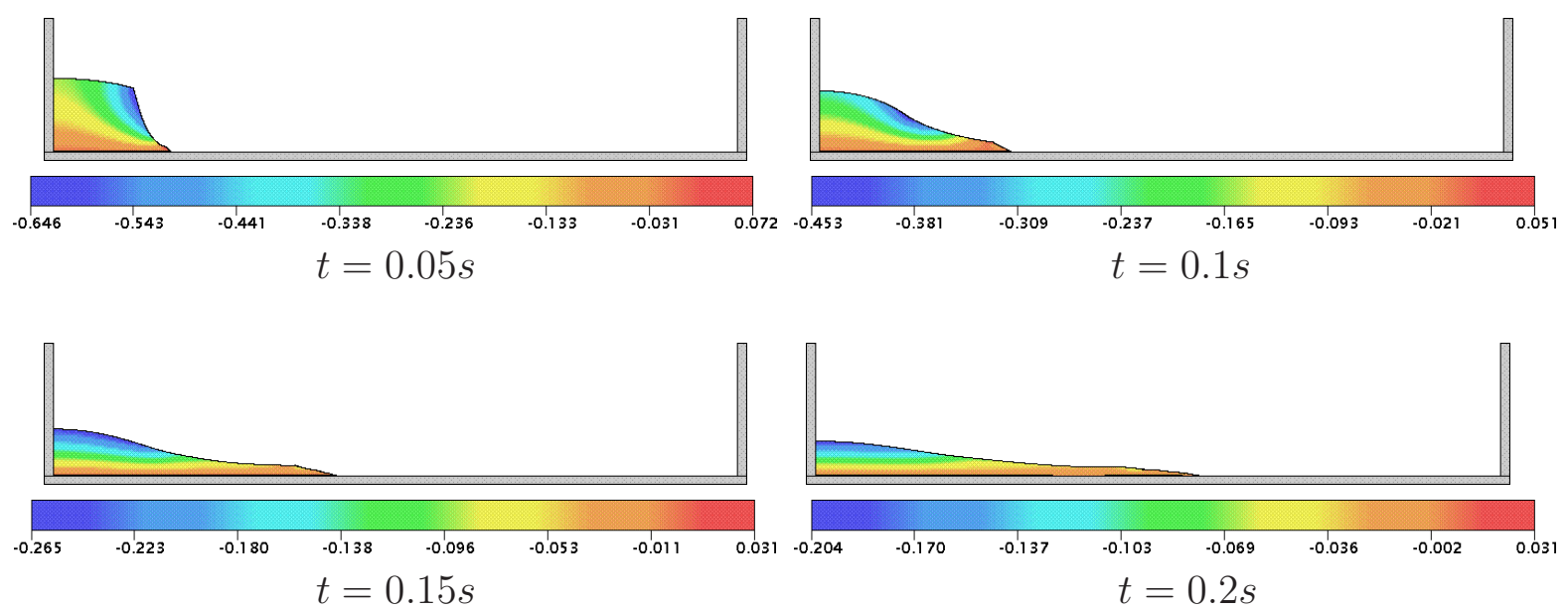

Figura 7.15: Resultados numéricos do esquema PFDPUS para a evolução da superfície livre do fluido e campo de velocidade na direção y nos tempos $t=0.05 \mathrm{~s}, t=0.1 \mathrm{~s}, t=0.15 \mathrm{~s}$ e $t=0.2 \mathrm{~s}$ para o problema do colapso de fluido.

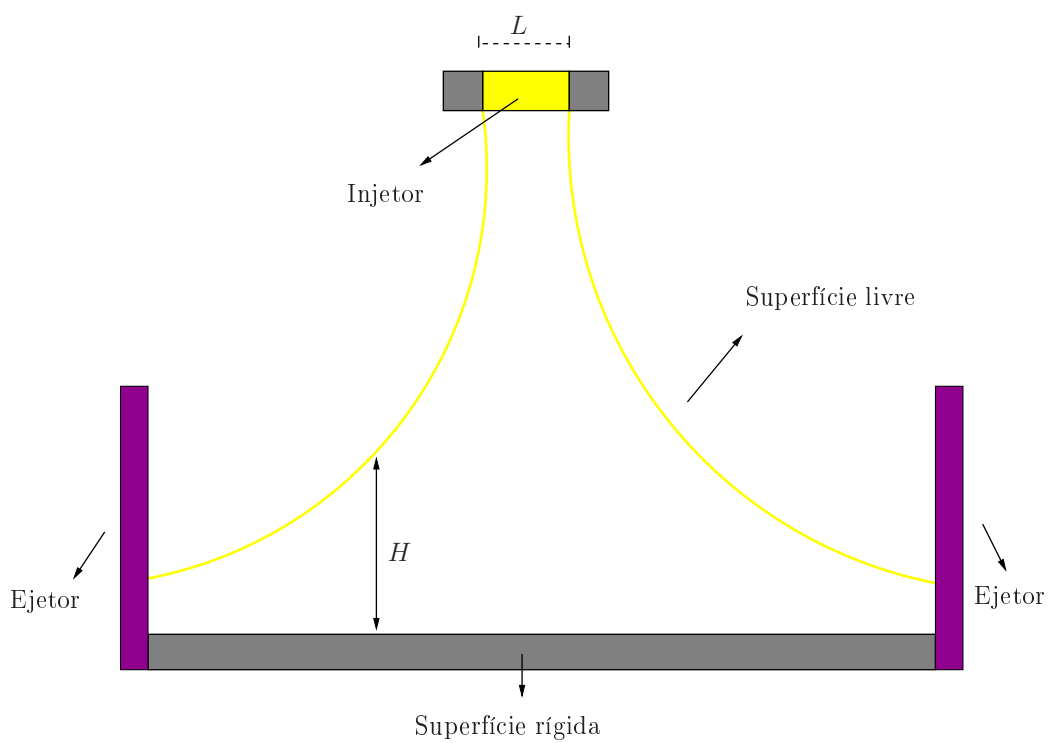

Figura 7.16: Representação esquemática do problema do jato livre sobre uma superfície rígida impermeável. 
Desprezando os efeitos da tensão superficial e admitindo que o escoamento está no estado estacionário, Watson [78] propôs uma solução analítica para a altura $H$ entre a superfície rígida e a superfície livre do fluido. Em resumo, a solução de Watson é

$$
H(x)= \begin{cases}\frac{\pi}{\sqrt{3}} \frac{\nu(x+l)}{Q}, & \text { se } x \geq x_{0} \\ a+\left(\frac{1-2 \pi}{3 \sqrt{3} c^{2}}\right) \delta(x), & \text { se } \quad x<x_{0}\end{cases}
$$

em que $Q=a U_{0}$ é a vazão, com $a$ o raio do injetor. Os parâmetros $x_{0}, l$ e $\delta$ são calculados por

$$
\begin{aligned}
& x_{0}=\left(\frac{3 \sqrt{3} c(\pi-c) \sqrt{3}}{2 \pi^{2}}\right) a R e, \\
& l=\left(\frac{3 \sqrt{3} c(2 \sqrt{3} c-\pi)}{2 \pi^{2}}\right) a R e,
\end{aligned}
$$

e

$$
\delta^{2}(x)=\frac{3 \sqrt{3} c^{3}}{2(\pi-c \sqrt{3})} \frac{\nu x}{U_{0}},
$$

onde a constante $c=1.402$ e $U_{0}$ é a velocidade de injeção.

Nesse problema, os resultados obtidos com o esquema PFDPUS, implementado no código Freeflow 2D [9], para a altura $H$ entre a superfície rígida e a superfície livre do fluido são comparados com a solução analítica de Watson [78] dada por (7.6). Para simulação, a condição no-slip é a adota na superfície rígida e os seguintes dados são empregados:

- Malha 1: $100 \times 25$ células computacionais $(\delta x=\delta y=0.001 m)$;

- Malha 2: $200 \times 50$ células computacionais $(\delta x=\delta y=0.0005 m)$;

- Malha 3: $400 \times 100$ células computacionais $(\delta x=\delta y=0.00025 m)$;

- Dimensão do domínio: $0.1 \mathrm{~m} \times 0.025 \mathrm{~m}$;

- Diâmetro do injetor: $L=0.004 m$;

- Escala de comprimento: $L_{0}=L=0.004 m$;

- Escala de velocidade (velocidade de injeção): $U_{0}=1 \mathrm{~m} / \mathrm{s}$;

- Coeficiente de viscosidade cinemática: $\nu=2 \times 10^{-6} \mathrm{~m}^{2} / \mathrm{s}$;

- Número de Reynolds: $R e=2000$.

Além disso, a distância entre o injetor e superfície rígida é 0.019m. A Figura 7.17 mostra a comparação entre a solução analítica de Watson [78] e os resultados numéricos obtidos com o esquema PFDPUS nas três malhas adotadas. Como pode ser visto nessa figura, os resultados numéricos concordam satisfatoriamente com a solução de Watson [78] e, com o refinamento da malha, as soluções numéricas obtidas se aproximam da solução analítica.

Nas Figuras 7.18, 7.19 e 7.20 são mostrados, respectivamente, as soluções numéricas obtidas 


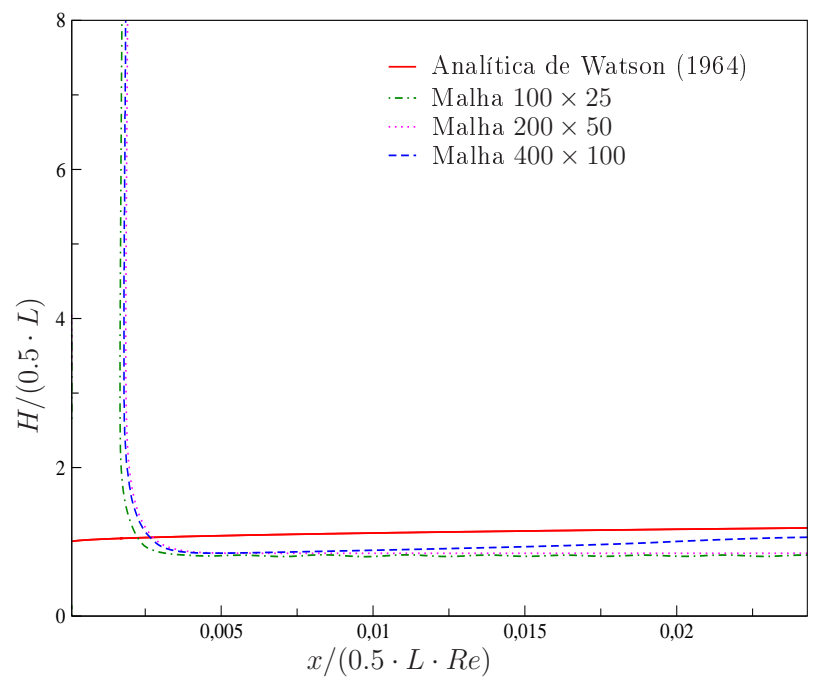

Figura 7.17: Comparação entre a solução analítica de Watson [78] e os resultados numéricos do esquema PFDPUS para a altura $H$ entre a superfície rígida e a superfície livre do fluido obtidos para o problema do jato livre.

pelo esquema PFDPUS (em uma malha de $400 \times 100$ células computacionais) para a evolução da superfície livre com respeito aos campos de pressão e velocidade (nas direções $x$ e $y$ ) nos tempos $t=0.03 s, t=0.04 s, t=0.05 s$ e $t=0.1 s$.

\section{Campo de pressão}
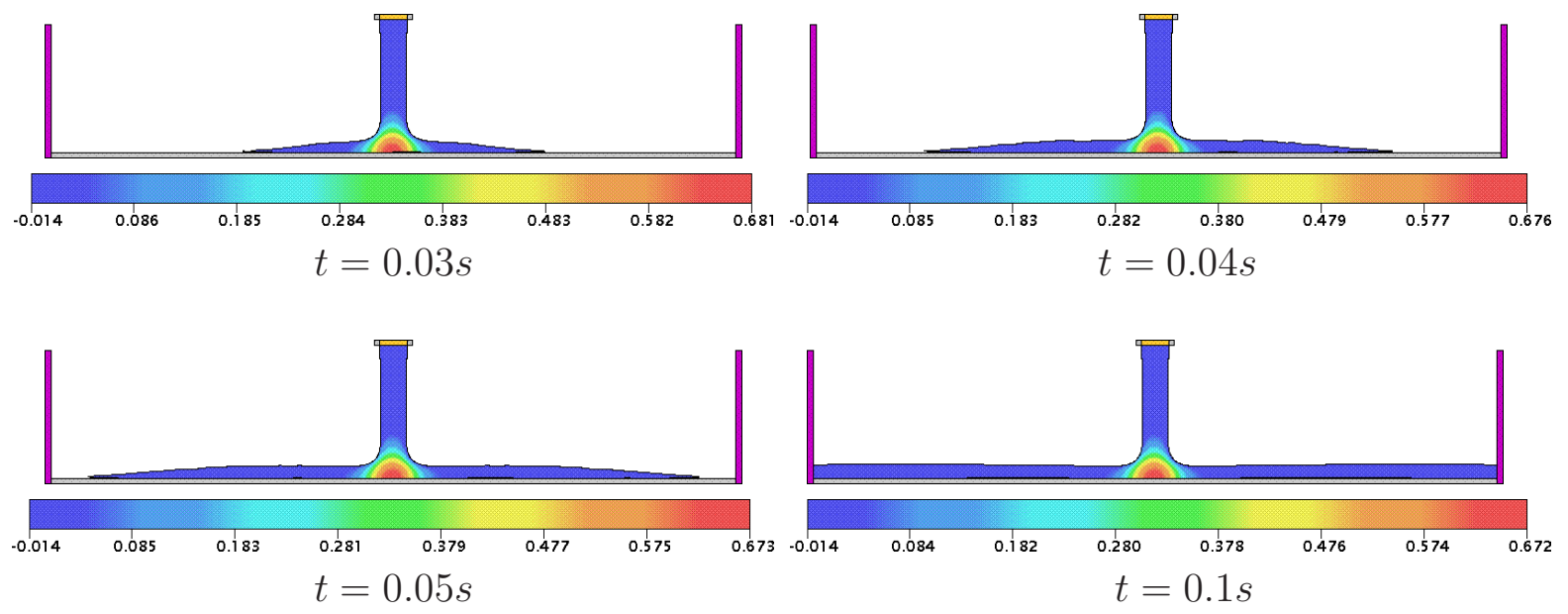

Figura 7.18: Resultados numéricos do esquema PFDPUS para a evolução da superfície livre e campo de pressão nos tempos $t=0.03 \mathrm{~s}, t=0.04 \mathrm{~s}, t=0.05 \mathrm{~s}$ e $t=0.1 \mathrm{~s}$ para o problema do jato livre. 


\section{Campo de velocidade na direção $x$}
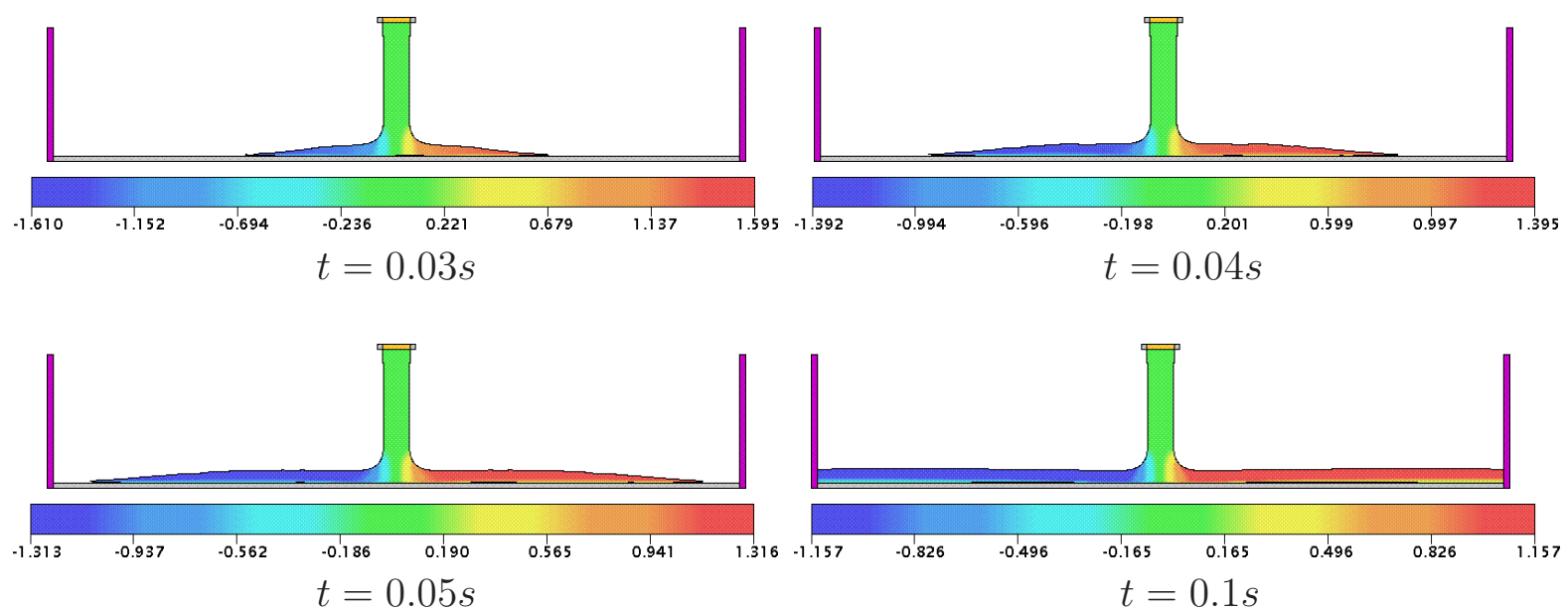

Figura 7.19: Resultados numéricos do esquema PFDPUS para a evolução da superfície livre e campo de velocidade na direção $x$ nos tempos $t=0.03 s, t=0.04 s, t=0.05 s$ e $t=0.1 s$ para o problema do jato livre.

\section{Campo de velocidade na direção $y$}
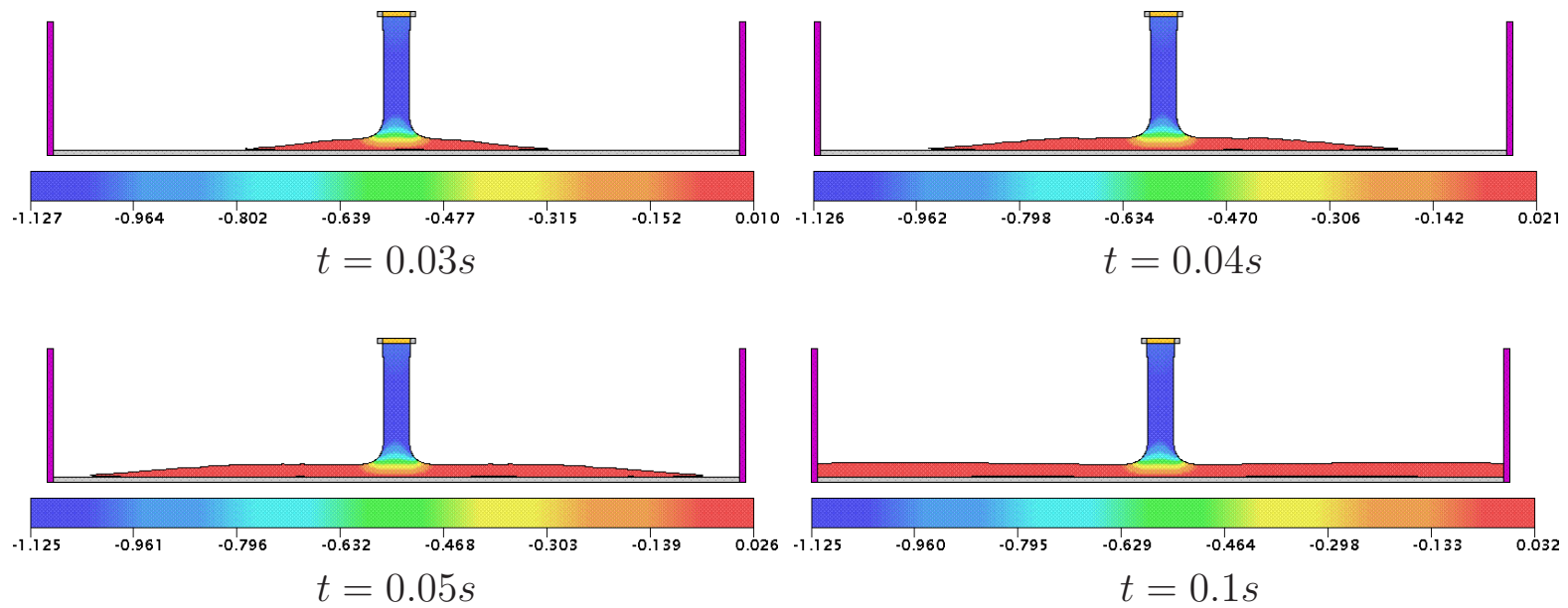

Figura 7.20: Resultados numéricos do esquema PFDPUS para a evolução da superfície livre e campo de velocidade na direção $y$ nos tempos $t=0.03 \mathrm{~s}, t=0.04 \mathrm{~s}, t=0.05 \mathrm{~s}$ e $t=0.1 \mathrm{~s}$ para o problema do jato livre.

\subsection{Escoamentos incompressíveis turbulentos 2D}

Nesta seção apresenta-se resultados numéricos para escoamentos de fluidos incompressíveis com superfícies livres móveis em regime turbulento. O problema considerado é o jato livre sobre uma superfície rígida impermeável, como está descrito na Subseção 7.2.2. Os resultados são gerados no ambiente de simulação Freeflow 2D [9], com a modelagem $\kappa-\varepsilon$ da turbulência e o esquema PFDPUS implementados.

Para o caso de um jato livre em regime turbulento, Watson [78] também apresenta uma solução analítica para a altura $H$ entre a superfície rígida e a superfície livre do fluido, a qual 
é usada para analisar o desempenho do esquema PFDPUS. A solução de Watson nesse caso é dada por (ver representação esquemática na Figura 7.16)

$$
H(x)= \begin{cases}\frac{81(7 A)^{\frac{1}{4}} k}{800}\left(\frac{\nu}{Q}\right)^{\frac{1}{4}}(x+l), & x \geq x_{0}, \\ a+\left(1-\frac{A}{k}\right) \delta(x), & x<x_{0}\end{cases}
$$

em que $Q=a U_{0}$ é a vazão, sendo $a$ o raio do injetor, $A=0.239$ e $k=0.26$. Ainda, tem-se que

$$
\begin{gathered}
x_{0}=\frac{320(9 A-2)}{81 \times 7^{\frac{1}{4}} A^{\frac{5}{4}}} a R e^{\frac{1}{4}} \\
l=\frac{160(1-2 A)}{9 \times 7^{\frac{1}{4}} A^{\frac{5}{4}}} a R e^{\frac{1}{4}}, \\
\delta(x)=\left(\frac{81}{320(9 A-2)}\right)^{\frac{4}{5}} 7^{\frac{1}{5}} k\left(\frac{a \nu}{Q}\right)^{\frac{1}{5}} x^{\frac{4}{5}} .
\end{gathered}
$$

Para simulação desse problema, a condição de contorno no-slip é aplicada na superfície rígida e os demais dados utilizados são:

- Malha 1: $200 \times 50$ células computacionais $(\delta x=\delta y=0.001 m)$;

- Malha 2: $400 \times 100$ células computacionais $(\delta x=\delta y=0.0005 m)$;

- Malha 3: $800 \times 200$ células computacionais $(\delta x=\delta y=0.00025 m)$;

- Dimensão do domínio: $0.2 m \times 0.05 m$;

- Diâmetro do injetor: $L=0.01 m$;

- Escala de comprimento: $L_{0}=L=0.01 m$;

- Escala de velocidade (velocidade de injeção): $U_{0}=1 \mathrm{~m} / \mathrm{s}$;

- Coeficiente de viscosidade cinemática: $\nu=2 \times 10^{-7} \mathrm{~m}^{2} / \mathrm{s}$;

- Número de Reynolds: $R e=50000$.

A distância entre o injetor e a superfície rígida é $0.015 m$. A Figura 7.21 mostra as comparações das soluções numéricas obtidas com as três malhas adotadas e a solução analítica de Watson em $t=1.5 \mathrm{~s}$. Observa-se que os resultados numéricos são satisfatórios e, com o refinamento da malha, que as soluções numéricas se aproximam cada vez mais da solução analítica, não apresentando quaisquer vestígios de oscilações em nenhuma das três malhas.

Para ilustração da evolução da superfície livre do fluido, as Figuras 7.22, 7.23 e 7.24 mostram as soluções numéricas obtidas pelo esquema PFDPUS (em uma malha de $800 \times 200$ células computacionais) com respeito aos campos de pressão e velocidade (nas direções $x$ e $y$ ) nos tempos $t=0.04 s, t=0.06 s, t=0.08 s$ e $t=0.15 s$. 


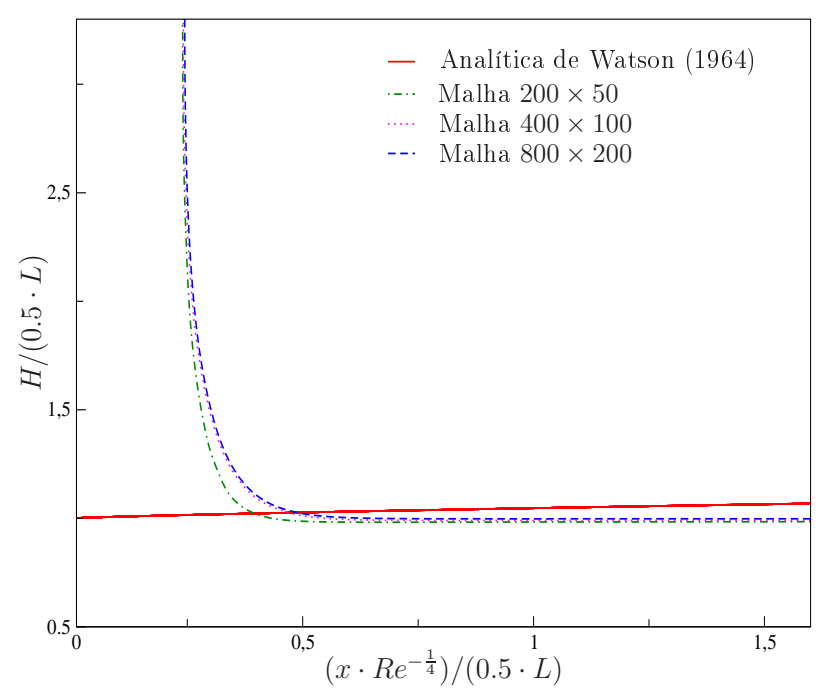

Figura 7.21: Comparação entre a solução analítica de Watson [78] e os resultados numéricos do esquema PFDPUS para a altura $H$ entre a superfície rígida e a superfície livre do fluido obtidos para o problema turbulento do jato livre.
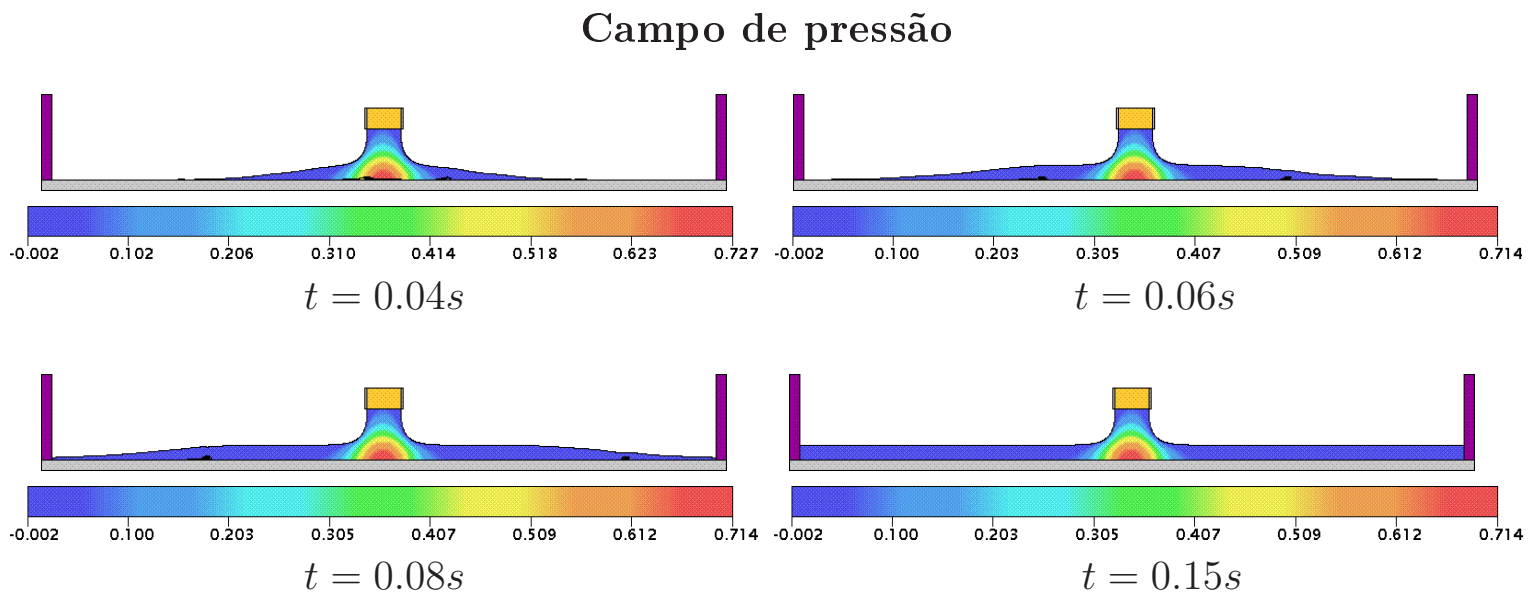

Figura 7.22: Resultados numéricos do esquema PFDPUS para a evolução da superfície livre e campo de pressão nos tempos $t=0.04 \mathrm{~s}, t=0.06 \mathrm{~s}, t=0.08 \mathrm{~s}$ e $t=0.15 \mathrm{~s}$ para o problema turbulento do jato livre. 


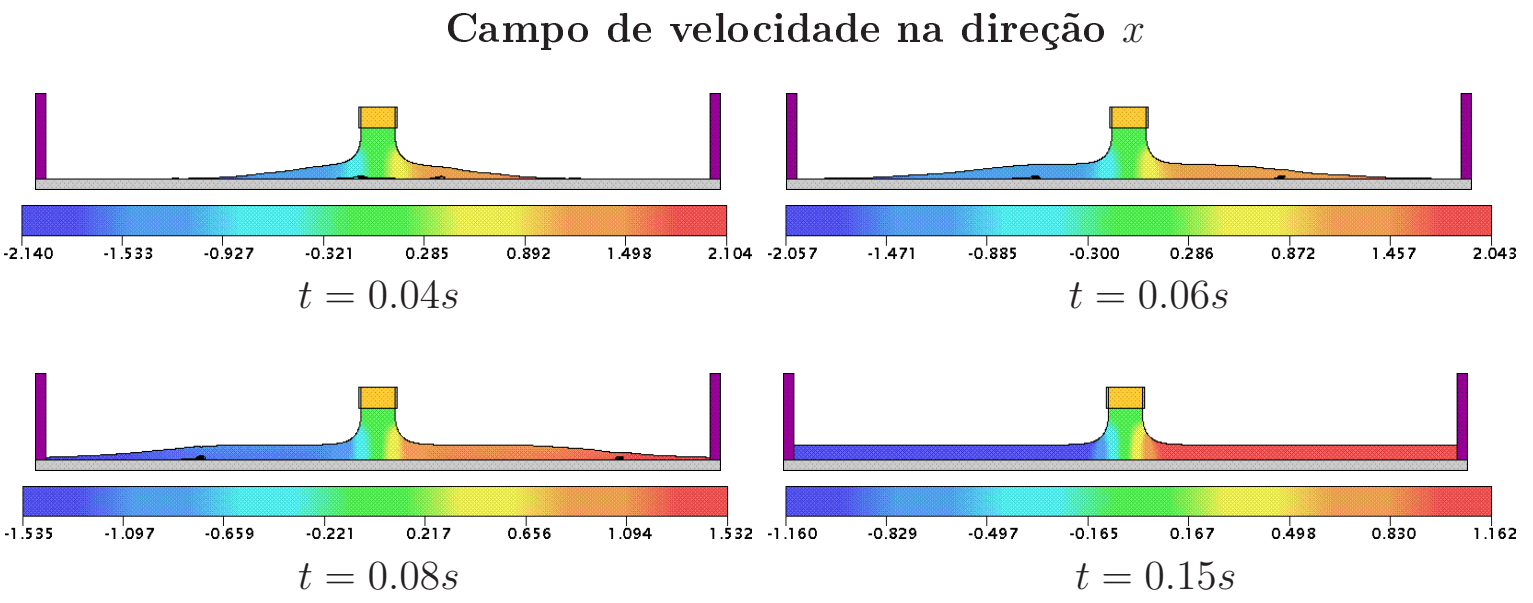

Figura 7.23: Resultados numéricos do esquema PFDPUS para a evolução da superfície livre e campo de velocidade na direção $x$ nos tempos $t=0.04 s, t=0.06 s, t=0.08 s$ e $t=0.15 s$ para o problema turbulento do jato livre.

\section{Campo de velocidade na direção $y$}
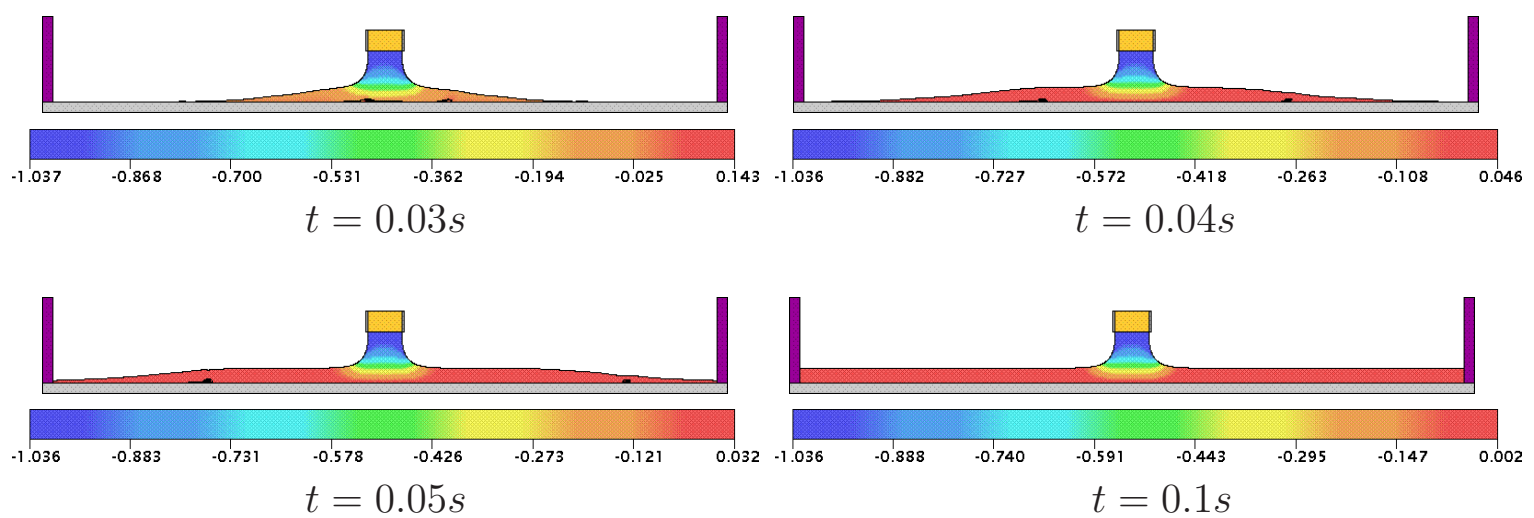

Figura 7.24: Resultados numéricos do esquema PFDPUS para a evolução da superfície livre e campo de velocidade na direção $y$ nos tempos $t=0.04 s, t=0.06 s, t=0.08 \mathrm{~s}$ e $t=0.15 \mathrm{~s}$ para o problema turbulento do jato livre.

\subsection{Escoamentos incompressíveis axissimétricos}

Nessa seção, estão contidos os resultados numéricos para escoamentos de fluidos incompressíveis laminares com superfícies livres móveis axissimétricos. As simulações são realizadas no ambiente de simulação Freeflow 2D [9] com simetria radial (ver mais detalhes Oliveira [16]), onde o esquema PFDPUS é implementado. Os problemas abordados nessa seção são o experimento de Taylor e o ressalto hidráulico circular.

\subsubsection{Experimento de Taylor}

Esse problema, estudado experimentalmente por Taylor [66], consiste em um jato de fluido que incide perpendicularmente sobre um recipiente contendo o mesmo fluido em repouso. O 
experimento de Taylor é utilizado aqui para uma comparação qualitativa com os resultados numéricos obtidos com o esquema PFPDPUS. A representação esquemática desse problema está ilustrada na Figura 7.25.

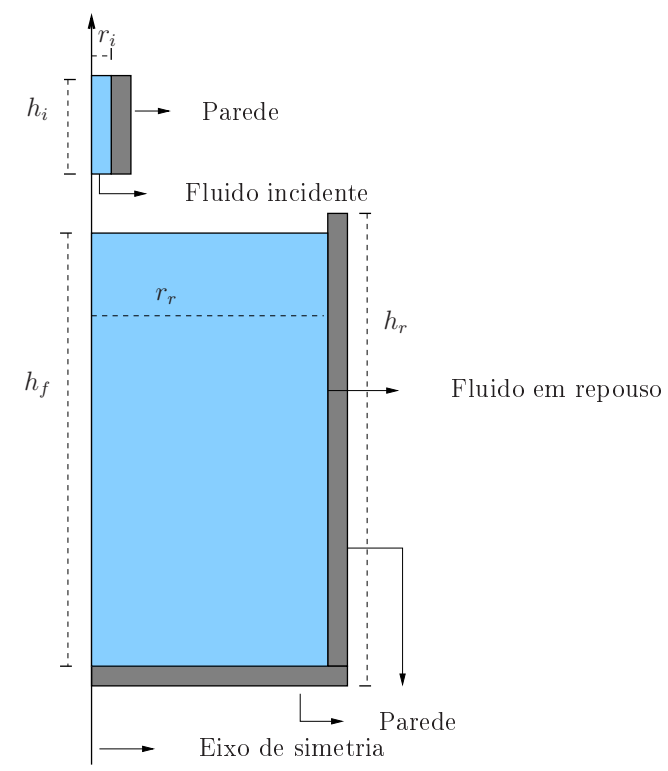

Figura 7.25: Representação esquemática do experimento de Taylor.

Para simulação utilizou-se o código Freeflow 2D [9] para problemas com simetria radial, equipado com o esquema PFDPUS. Além disso, adota-se a condição de contorno no-slip e demais dados para simulação:

- Malha: $123 \times 403$ células computacionais $(\delta x=\delta y=0.0005 m)$;

- Dimensão do domínio: $0.0615 m \times 0.2015 m$;

- Raio do injetor: $r_{i}=0.002 m$;

- Altura do injetor: $h_{i}=0.03 m$;

- Raio do recipiente cilindrico: $r_{r}=0.06 m$;

- Altura do recipiente cilindrico: $h_{r}=0.17 m$;

- Altura do fluido dentro do recipiente: $h_{f}=0.16 m$;

- Escala de comprimento: $L=2 r_{i}=0.004 m$;

- Escala de velocidade (velocidade de injeção): $U_{0}=0.5 \mathrm{~m} / \mathrm{s}$;

- Coeficiente de viscosidade cinemática: $\nu=10^{-5} \mathrm{~m}^{2} / \mathrm{s}$;

- Número de Reynolds: $R e=200$.

O injetor está localizado a $0.1 \mathrm{~m}$ do fluido. A Figura 7.26 mostra a comparação entre o experimento de Taylor e os resultados numéricos obtidos com o esquema PFDPUS nos tempos $t=0.25 \mathrm{~s}$ e $t=2.5 \mathrm{~s}$, respectivamente. Observa-se que quando o jato de fluido atinge a superfície rígida $(t=2.5 \mathrm{~s})$ este forma uma estrutura toroidal, revelando uma ótima coerência dos resultados numéricos com os dados experimentais apresentados por Taylor [66]. 

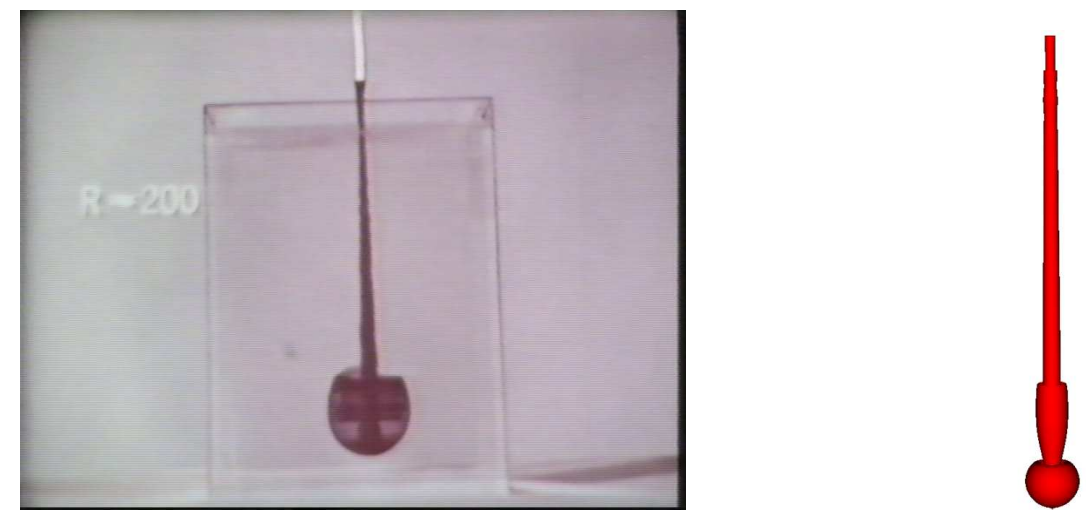

$t=0.75 \mathrm{~s}$
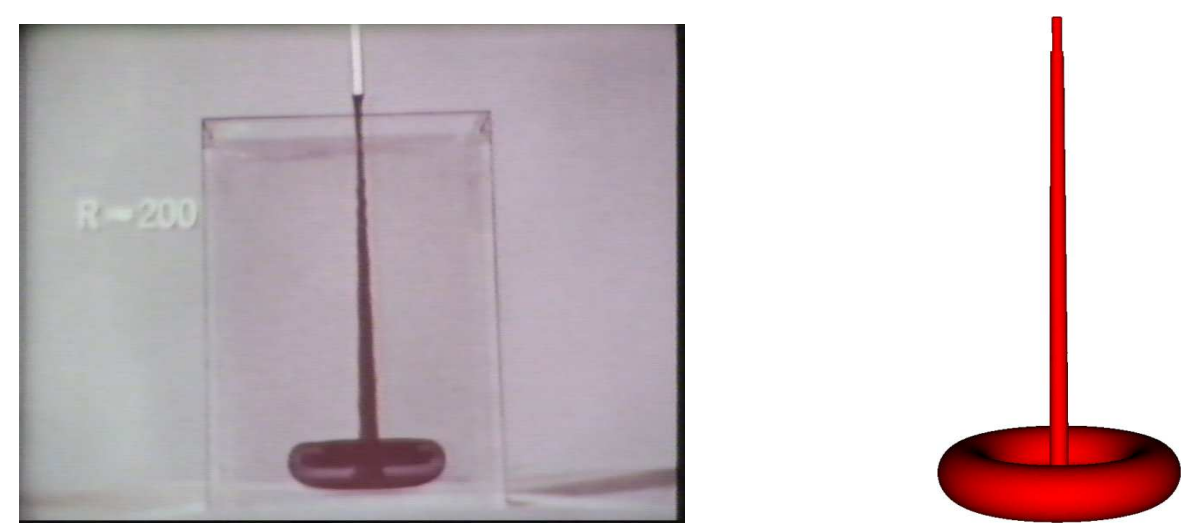

$t=2.5 s$

Figura 7.26: Comparação entre os resultados experimentais e numéricos para o experimento de Taylor obtidos com o esquema PFDPUS nos tempos $t=0.75 \mathrm{~s}$ e $t=2.5 \mathrm{~s}$, respectivamente.

Para complementar, a Figura 7.27 ilustra os resultados numéricos obtidos com o esquema PFDPUS em $t=10 \mathrm{~s}$. Essa figura apresenta a estrutura completa simulada pelo esquema (Figura 7.27-(a)) e, também, um corte (Figura 7.27-(b)) em que possível observar em detalhes a formação dos vórtices capturados na simulação. Os resultados numéricos para os campos de pressão e velocidade (nas direções $r$ e $z$ ) estão mostrados na Figura 7.28.

\subsubsection{Ressalto hidráulico circular}

Um fenômeno bastante visto no dia-a dia, o ressalto hidráulico circular ocorre quando um jato circular de água incide perpendicularmente numa superfície rígida e se espalha radialmente a partir do ponto de impacto. Segundo a literatura (veja, por exemplo, [37] e [65]), durante o espalhamento a espessura decresce e, então, a uma certa distância cresce repentinamente sob à ação de um gradiente de pressão adverso. Esse gradiente pode causar separação da película em alguma distância radial, levando à formação de um fenômeno interessante conhecido como ressalto hidráulico circular. A Figura 7.29 mostra a representação esquemática desse fenômeno.

Uma solução analítica para a espessura da camada de fluido foi proposta por Watson [78] e 


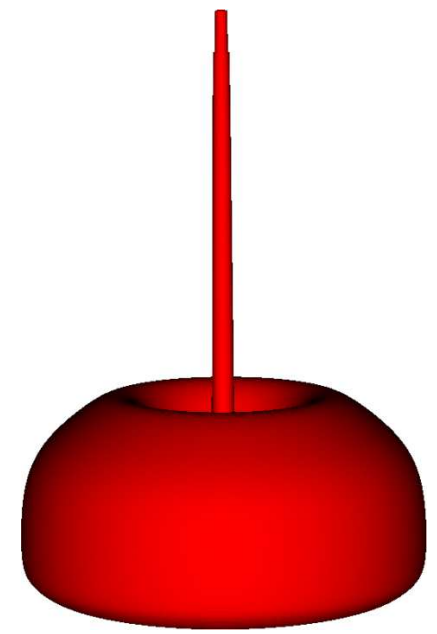

(a) Estrutura completa

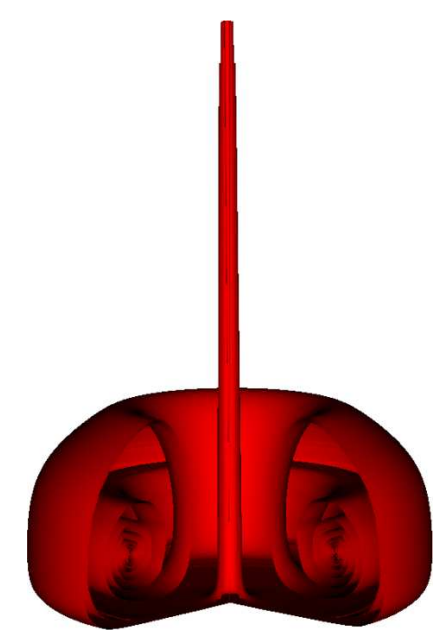

(b) Estrutura com corte

Figura 7.27: Resultados numéricos do esquema PFDPUS para o experimento de Taylor em $t=10 s$.

é dada da forma

$$
H(r)= \begin{cases}\frac{a^{2}}{2 r}+\left(1-\frac{2 \pi}{3 \sqrt{3} c^{2}}\right) \delta, & \text { se } r<r_{0} \\ \frac{2 \pi^{2}}{3 \sqrt{3}} \frac{\nu\left(r^{3}+l^{2}\right)}{Q r}, & \text { se } r \geq r_{0}\end{cases}
$$

em que

$$
\delta^{2}=\frac{\pi \sqrt{3} c^{3}}{\pi-c \sqrt{3}} \frac{\nu r a^{2}}{Q}
$$

onde $c=1.402, r_{0}=0.3155 a R e^{\frac{1}{3}}$ e $l$ é uma constante arbitrária, a qual é calculada considerando o desenvolvimento inicial da camada limite $\delta$, sendo $l=0.567 a R e^{\frac{1}{3}}$. Vale ressaltar que esta solução analítica é válida somente na região do domínio em que o fluido já atingiu a superfície rígida, longe do ponto de impacto e antes da formação do ressalto hidráulico.

Para a simulação, o código Freeflow 2D [9] para problemas com simetria radial, equipado com o esquema PFDPUS, é empregado. Os resultados numéricos obtidos são comparados com a solução analítica de Watson [78] dada por (7.14) e com dados experimentais (ver Middleman [49]). Utiliza-se condição de contorno no-slip e os seguintes dados para a simulação:

- Malha 1: $200 \times 126$ células computacionais $(\delta x=\delta y=0.00025 m)$;

- Malha 2: $400 \times 252$ células computacionais $(\delta x=\delta y=0.000125 m)$;

- Malha 3: $800 \times 504$ células computacionais $(\delta x=\delta y=0.0000625 m)$;

- Dimensão do domínio: $0.05 m \times 0.0315 m$;

- Raio do injetor: $r_{i}=0.004 m$;

- Altura do injetor: $h_{i}=0.00075 m$; 

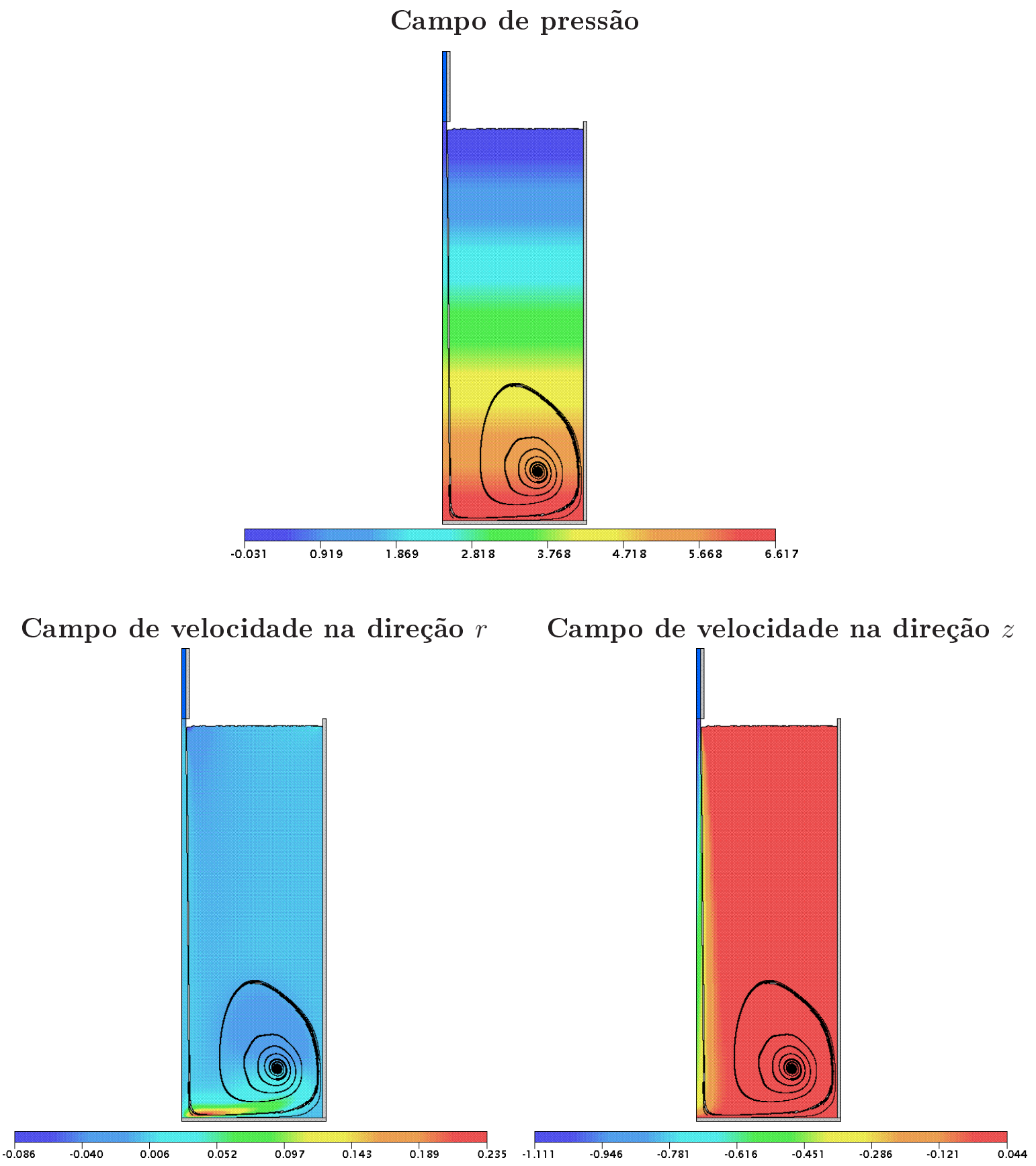

Figura 7.28: Resultados numéricos do esquema PFDPUS para a evolução da superfície livre e campos de pressão e velocidade (na direção $r$ e $z$ ) no tempo $t=10 \mathrm{~s}$ para o problema do experimento de Taylor.

- Escala de comprimento: $L_{0}=2 r_{i}=0.008 m$;

- Escala de velocidade (velocidade de injeção): $U_{0}=0.375 \mathrm{~m} / \mathrm{s}$;

- Coeficiente de viscosidade cinemática: $\nu=1.2 \times 10^{-5} \mathrm{~m}^{2} / \mathrm{s}$;

- Número de Reynolds: $R e=250$.

Aqui, o injetor está posicionado a uma distância $0.03075 \mathrm{~m}$ da superfície rígida. A compara- 


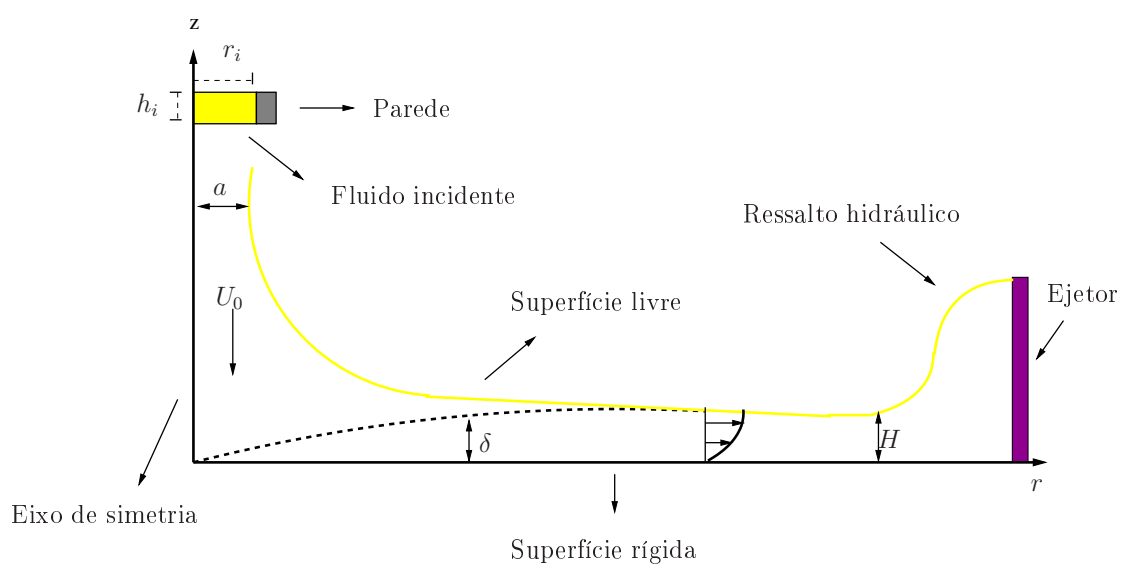

Figura 7.29: Representação esquemática do ressalto hidráulico circular.

ção entre a solução analítica de Watson [78] e os resultados numéricos com o esquema PFDPUS, nas três malhas adotadas, para a espessura da camada de fluido é mostrada na Figura $7.30 \mathrm{em}$ $t=2.5 \mathrm{~s}$. É possível notar que as soluções numéricas estão em boa concordância com a solução de Watson na região onde a solução analítica é válida.

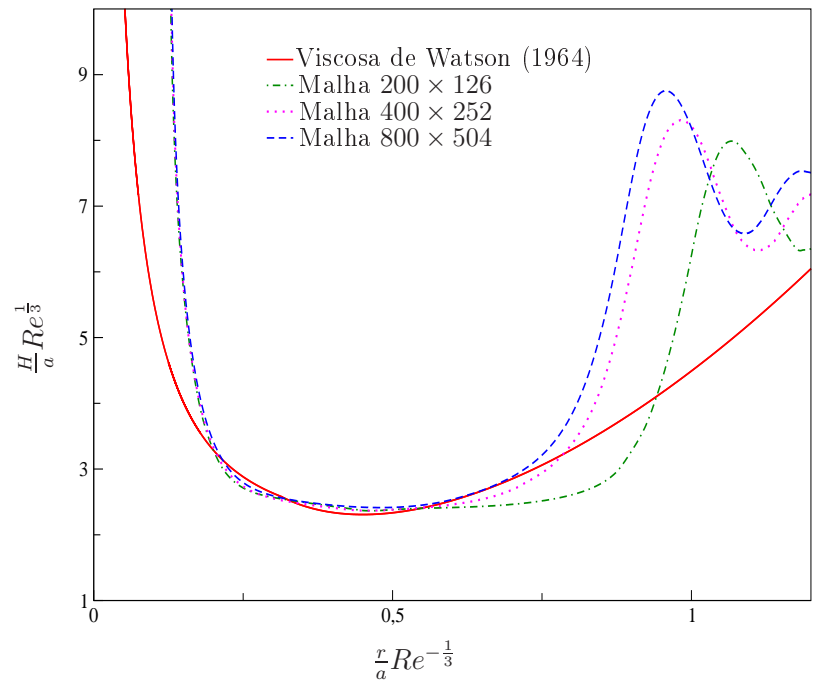

Figura 7.30: Comparação entre a solução viscosa de Watson [78] e os resultados numéricos do esquema PFDPUS para a espessura $H$ da camada de fluido.

Para efeito de comparação qualitativa, os resultados numéricos obtidos para esse fenômeno em $t=2.5 s$ são comparados com dados experimentais, como mostrado na Figura 7.31. Observa-se claramente que a simulação concorda com os dados experimentais, fornecendo uma boa captura do fenômeno do ressalto hidráulico circular. 


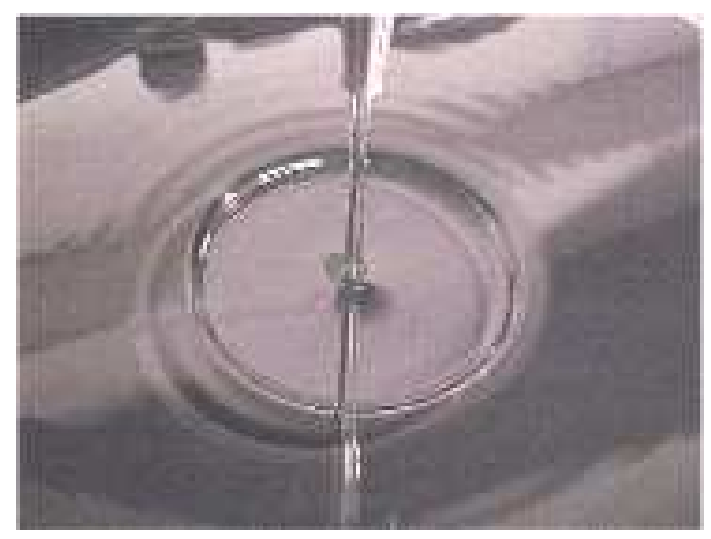

(a) Experimento

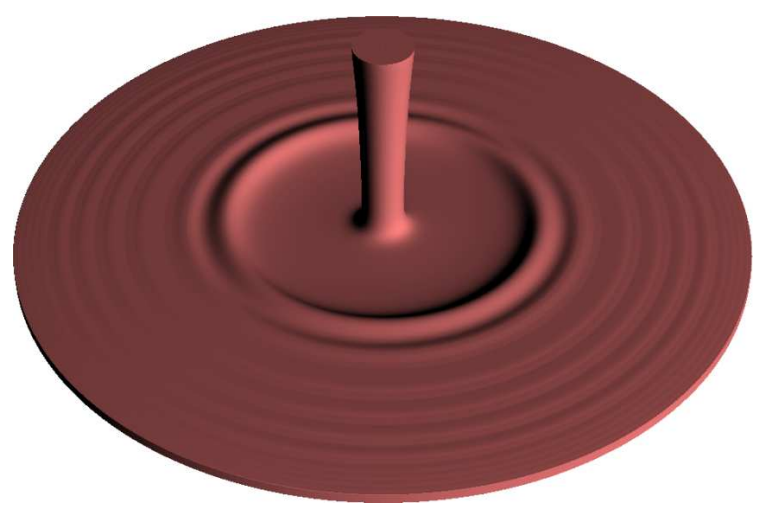

(b) PFDPUS

Figura 7.31: Comparação entre o resultados experimental e o numérico obtido com o esquema PFDPUS para o ressalto hidráulico circular em $t=2.5 \mathrm{~s}$. 
CAPÍTUlO

\section{8}

\section{Resultados numéricos 3D}

Este capítulo engloba resultados numéricos para escoamentos incompressíveis com superfícies livres móveis modelados pelas equações de Navier-Stokes 3D. Usando-se o ambiente de simulação Freeflow 3D de Castelo et al. [9], equipado com o esquema PFDPUS, apresentam-se resultados numéricos para os fenômenos conhecidos como jato oscilante e ressalto hidráulico circular. É bom lembrar que os testes apresentados aqui são simulados com o esquema PFDPUS com o par de parâmetros $\alpha=10$ e $\beta=100$.

\subsection{Jatos oscilantes}

A compreensão dos fenômenos de jatos oscilantes (jet buckling) tem sido um dos principais temas de pesquisa em dinâmica dos fluidos nos últimos 40 anos (ver, por exemplo, [70]). Esse problema é constantemente observado no cotidiano e tem várias aplicações industriais tais como na indústria de alimentos e processamento de polímeros, entre outras. O fenômeno é caracterizado principalmente por instabilidades físicas que surgem quando um jato de fluido altamente viscoso colide contra uma superfície rígida impermeável. Ao aderirem à superfície rígida, esses jatos formam dobras ou espirais e, por essa razão, são denominados jatos oscilantes.

Cruickshank [13], por meio de seus estudos experimentais, tentou determinar os parâmetros orientadores do surgimento desse fenômeno. Ele observou que o jato oscilante circular ocorre para determinados valores críticos de dois parâmetros (demonstrados posteriormente por Tomé e McKee [70]), os quais são definidos pelas condições

$$
R e \leq 1.2 \quad \text { e } \quad \frac{H}{d}>7.2
$$

onde $H$ é a distância entre o injetor e a superfície rígida e $d$ é o diâmetro do injetor.

O objetivo aqui é mostrar que o Freeflow 3D atual (equipado com o esquema PFDPUS) 
é também capaz de simular com sucesso essas instabilidades físicas em jatos a baixo número de Reynolds. É importante observar nesse ponto que, muito embora o esquema PFDPUS foi proposto para problemas em que a convecção é dominante, ele também é útil para simular problemas a Reynolds menor que 1. Uma representação esquemática do problema para os casos circular e planar está ilustrada na Figura 8.1.

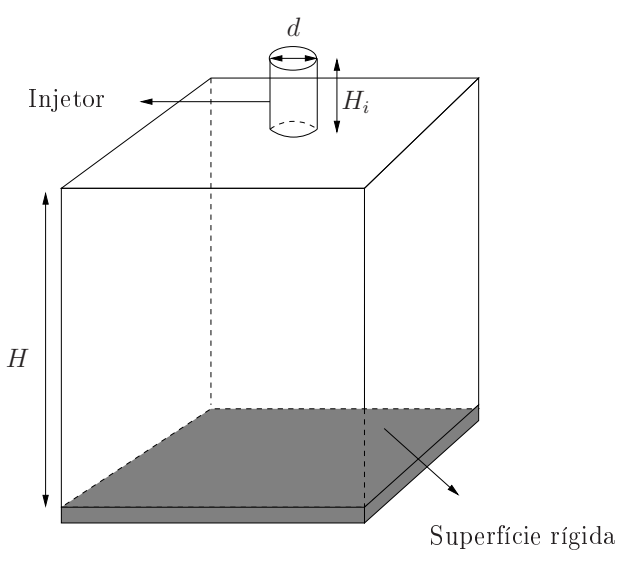

(a) Circular

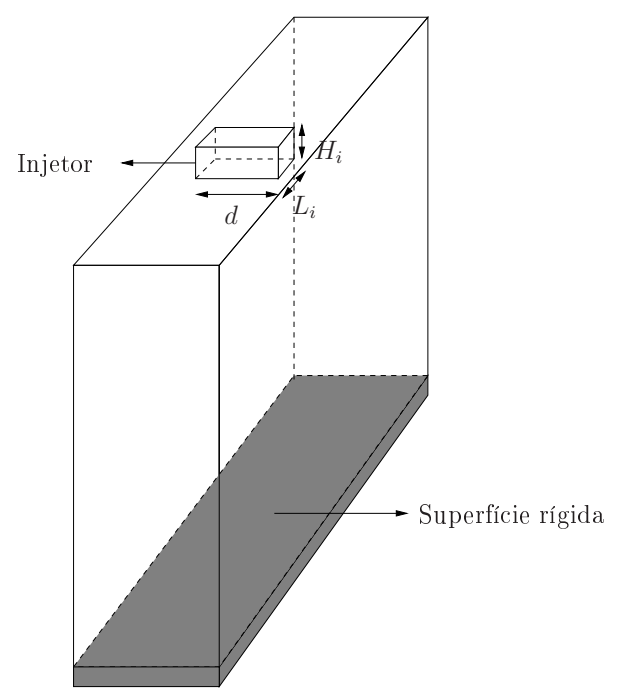

(b)Planar

Figura 8.1: Representação esquemática dos jatos oscilantes circular e planar.

\section{Problema 1: (Jato oscilante circular)}

Para este problema consideram-se dois casos, os quais estão descritos a seguir.

Caso 1: Para este teste é utilizado o código Freeflow 3D [9] onde o esquema PFDPUS é implementado. A condição no-slip é aplicada na superfície rígida e demais dados para simulação são empregados:

- Malha: $50 \times 50 \times 63$ células computacionais $(\delta x=\delta y=\delta z=0.02 m)$;

- Dimensão do domínio: $1 m \times 1 m \times 1.26 m$;

- Diâmetro do injetor $d=0.08 m$;

- Altura do injetor: $H_{i}=0.2 m$;

- Distância entre o injetor e a superfície rígida: $H=1 \mathrm{~m}$;

- Escala de comprimento: $L_{0}=d=0.08 m$;

- Escala de velocidade (velocidade de injeção): $U_{0}=1 \mathrm{~m} / \mathrm{s}$;

- Coeficiente de viscosidade cinemática: $\nu=0.278 \mathrm{~m}^{2} / \mathrm{s}$;

- Número de Reynolds: $R e=0.288$.

É importante observar que as condições de Cruickshank (8.1) para a formação do fenômeno (buckling) estão satisfeitas, uma vez que, $R e=0.288$ e $\frac{H}{d}=12.5$. Os resultados obtidos com 
o esquema PFDPUS em diferentes tempos $(t=1 s, t=3 s, t=5 s, t=7 s, t=9 s, t=10 s$, $t=12 \mathrm{~s}, t=15 \mathrm{~s}$ e $t=17 \mathrm{~s}$ ) podem ser vistos na Figura 8.2. Observa-se claramente por essas figuras que o fenômeno do jato oscilante circular é simulado com sucesso ao se usar o esquema PFDPUS.

Caso 2: Este teste é também realizado no código Freeflow 3D [9] no qual o esquema PFDPUS é implementado. Aqui, considera-se o diâmetro do injetor como a metade do escolhido no caso anterior. Considera-se a condição no-slip na superfície rígida e os seguintes dados para simulação:

- Malha: $100 \times 100 \times 123$ células computacionais $(\delta x=\delta y=\delta z=0.01 m)$;

- Dimensão do domínio: $1 m \times 1 m \times 1.23 m$;

- Diâmetro do injetor $d=0.04 m$;

- Altura do injetor: $H_{i}=0.2 m$;

- Distância entre o injetor e a superfície rígida: $H=1 \mathrm{~m}$;

- Escala de comprimento: $L_{0}=2 d=0.08 m$;

- Escala de velocidade (velocidade de injeção): $U_{0}=1 \mathrm{~m} / \mathrm{s}$;

- Coeficiente de viscosidade cinemática: $\nu=0.278 \mathrm{~m}^{2} / \mathrm{s}$;

- Número de Reynolds: $R e=0.288$.

Nota-se também nesse caso que as condições de Cruickshank (8.1) estão satisfeitas, pois $R e=0.288$ e $\frac{H}{d}=25$. A Figura 8.3 mostra a comparação qualitativa entre os resultados experimentais (ver Nóbrega et al. [51]) e os resultados numéricos obtidos com o esquema FPDPUS em quatro tempos $(t=1.5 s, t=2.8 \mathrm{~s}, t=4.7 \mathrm{~s}$ e $t=9.4 \mathrm{~s})$. Pode-se observar que, mesmo tomando o diâmetro do injetor mais fino, o esquema PFDPUS captura muito bem o fenômeno do jato oscilante circular e apresenta boa concordância com os dados experimentais.

\section{Problema 2: (Jato oscilante planar)}

Neste segundo problema, uma geometria retangular é adotada para o injetor (ver Figura 8.1). O objetivo é verificar o desempenho do esquema PFDPUS na captura da formação das dobras no decorrer do escoamento. A condição no-slip é aplicada na superfície rígida e os parâmetros envolvidos na simulação são:

- Malha: $146 \times 66 \times 213$ células computacionais $(\delta x=\delta y=\delta z=0.005 m)$;

- Dimensão do domínio: $0.73 m \times 0.33 m \times 1.065 m$;

- Dimensões injetor: $d=0.2 m, L_{i}=0.02 m$ e $H_{i}=0.05 m$;

- Distância entre o injetor e a superfície rígida: $H=1 \mathrm{~m}$;

- Escala de comprimento: $L_{0}=d=0.2 m$;

- Escala de velocidade (velocidade de injeção): $U_{0}=1 \mathrm{~m} / \mathrm{s}$;

- Coeficiente de viscosidade cinemática: $\nu=0.278 \mathrm{~m}^{2} / \mathrm{s}$;

- Número de Reynolds: $R e=0.72$. 


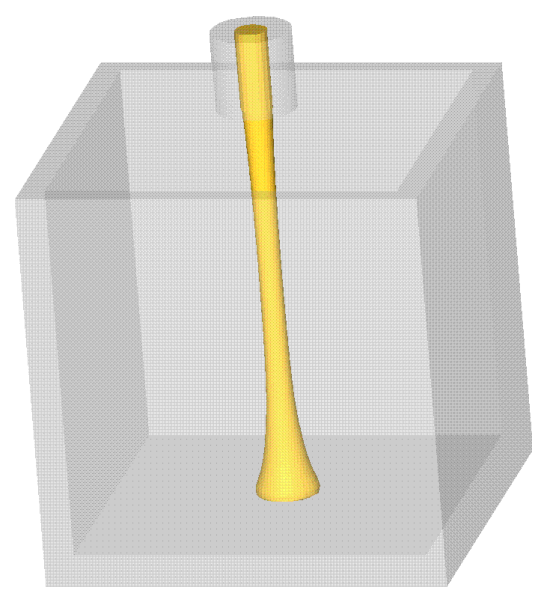

$t=1 s$

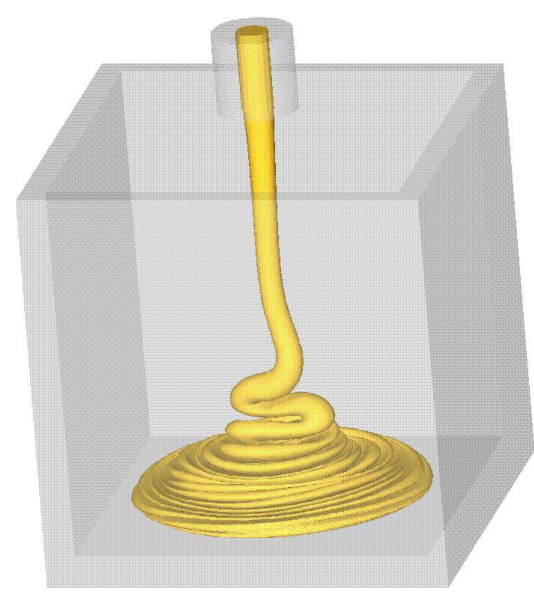

$t=7 \mathrm{~s}$

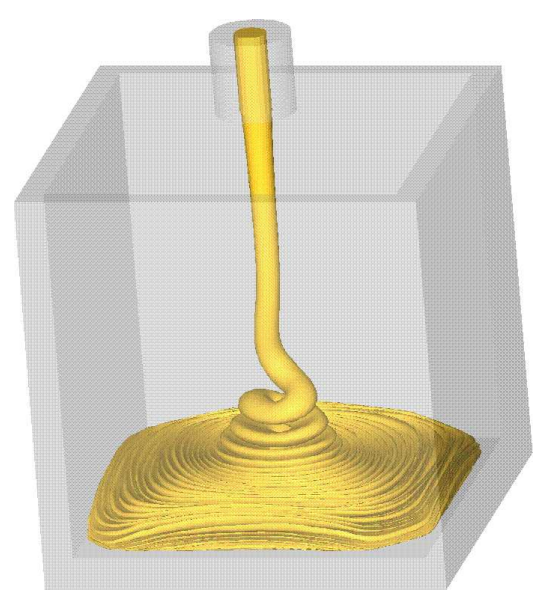

$t=13 \mathrm{~s}$

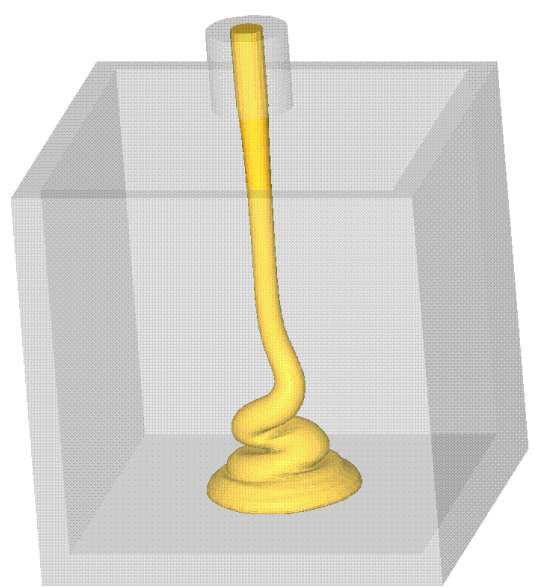

$t=3 \mathrm{~s}$

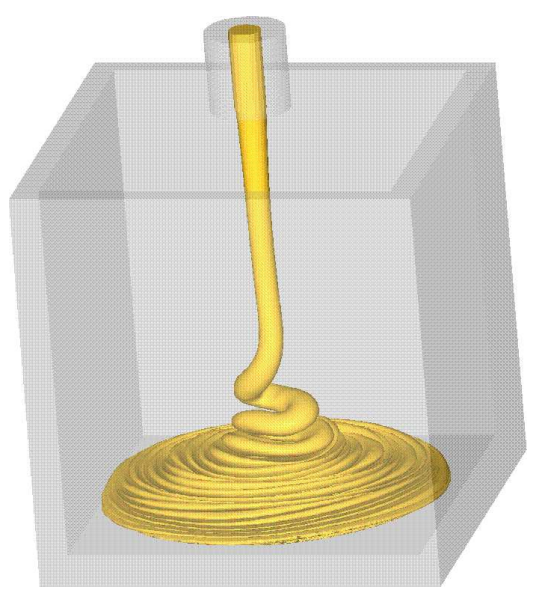

$t=9 \mathrm{~s}$

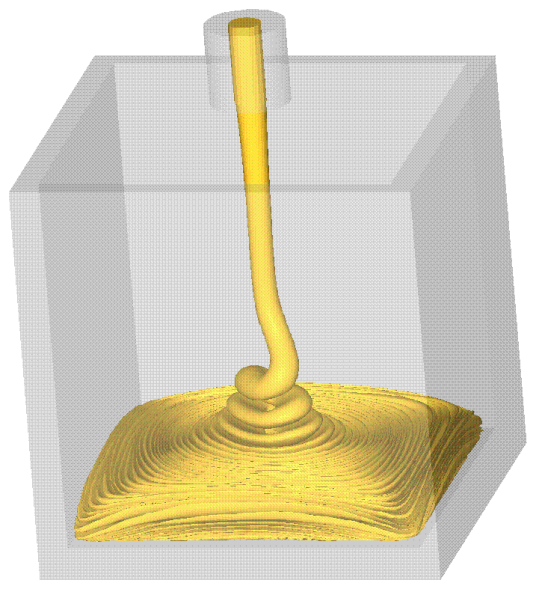

$t=15 \mathrm{~s}$

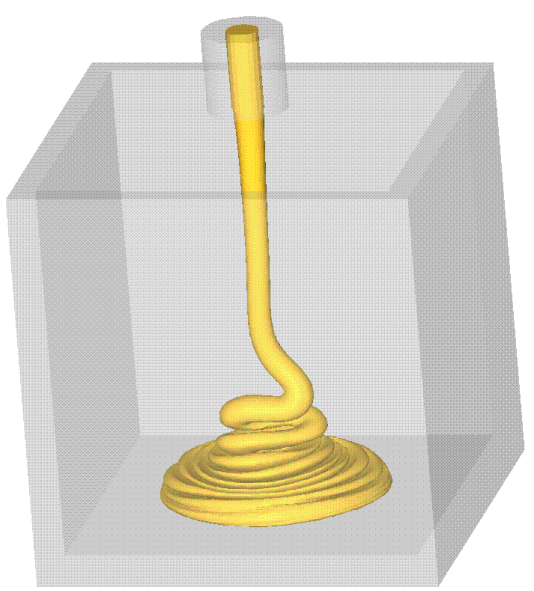

$t=5 \mathrm{~s}$

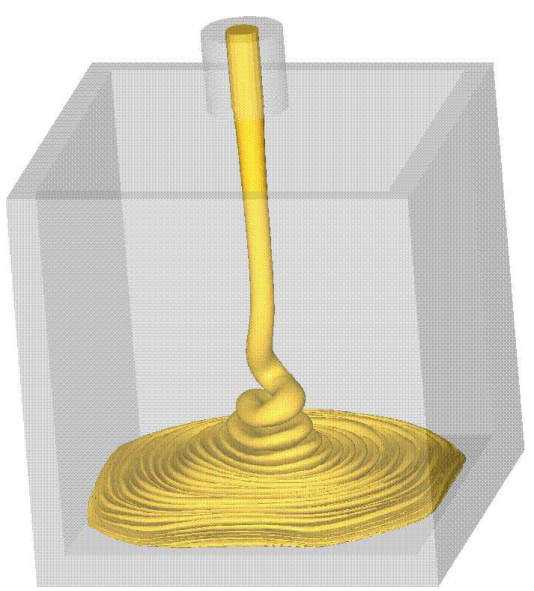

$t=11 \mathrm{~s}$

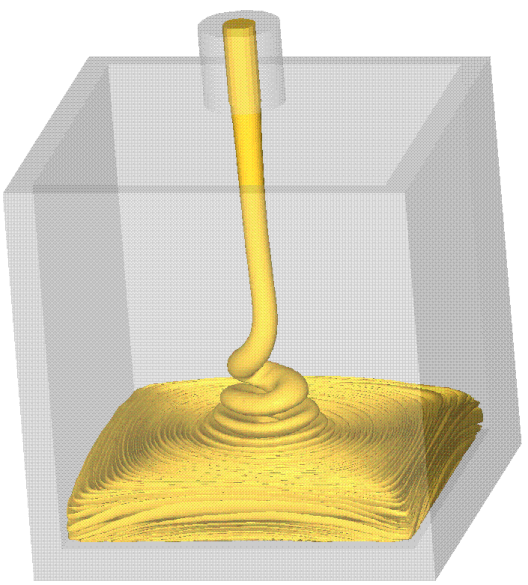

$t=17 \mathrm{~s}$

Figura 8.2: Resultados numéricos para o jato oscilante circular obtidos com o esquema PFDPUS nos tempos $t=1 s, t=3 s, t=5 s, t=7 s, t=9 s, t=11 s, t=15 s$ e $t=17 s$. 

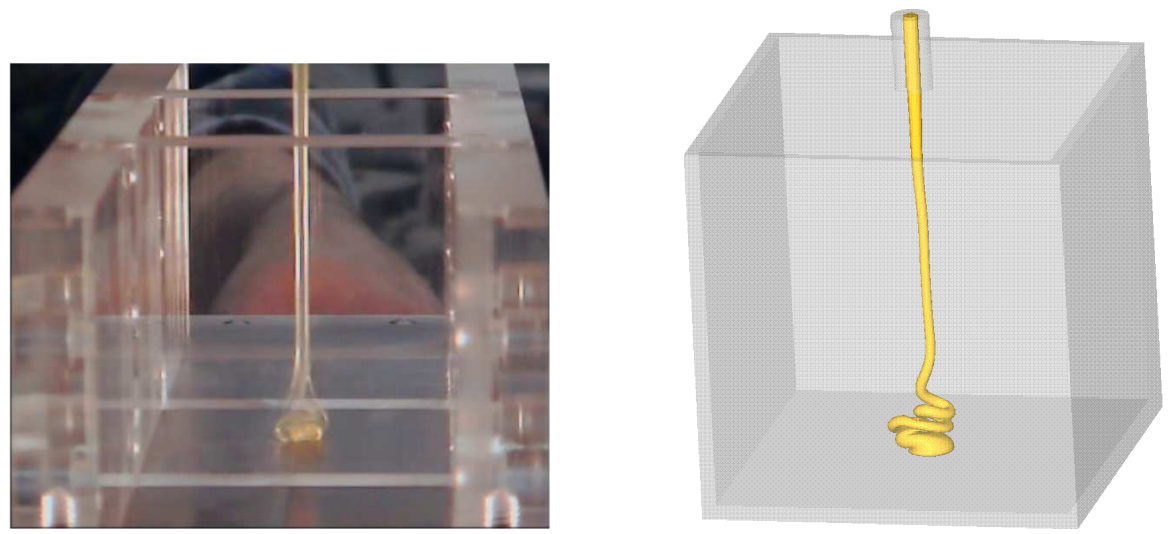

$t=1.5 \mathrm{~s}$
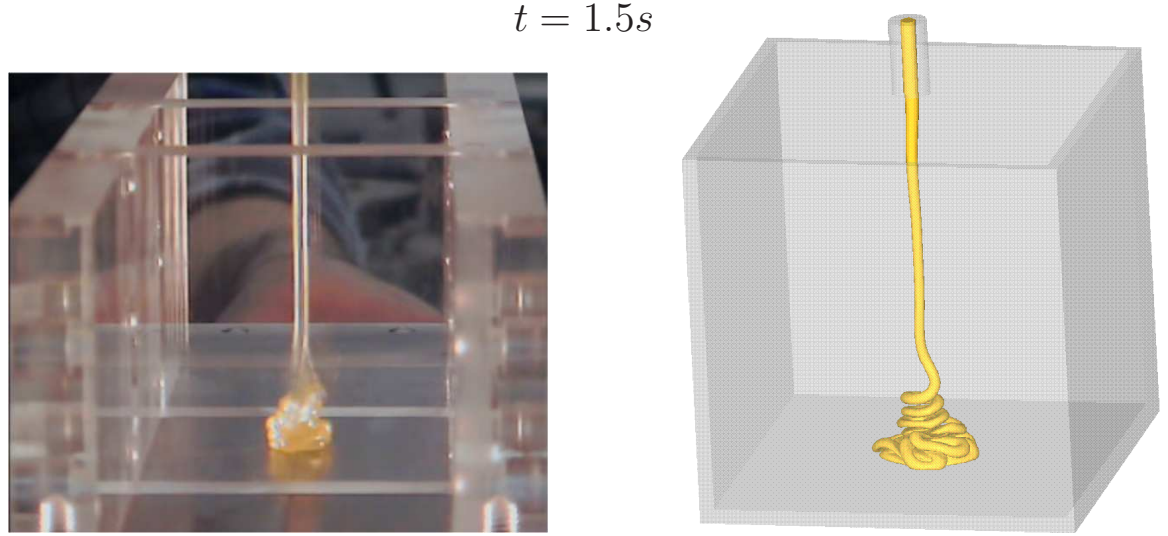

$t=2.8 s$
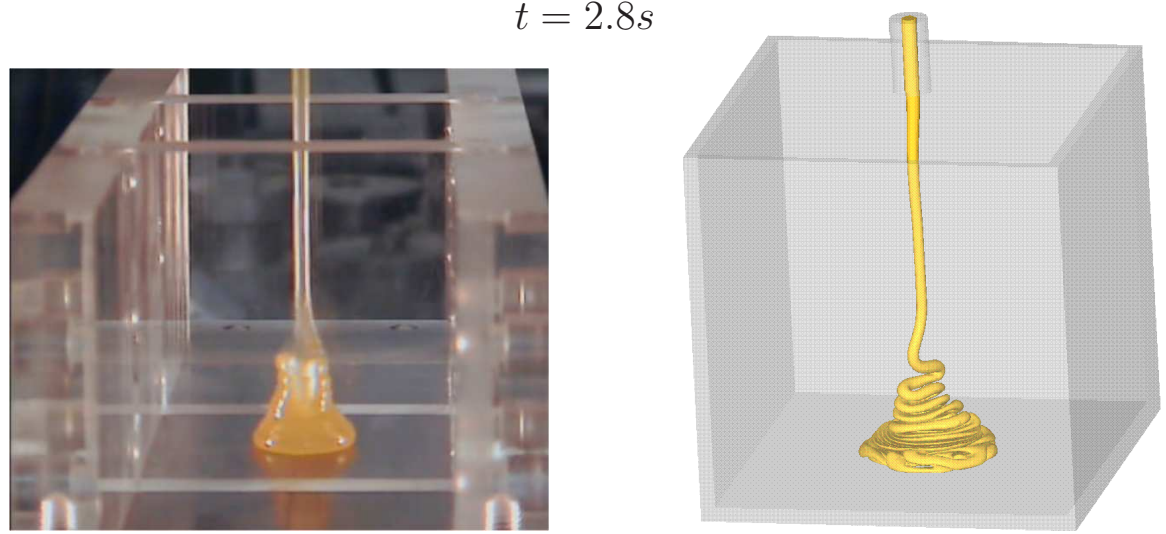

$t=4.7 \mathrm{~s}$
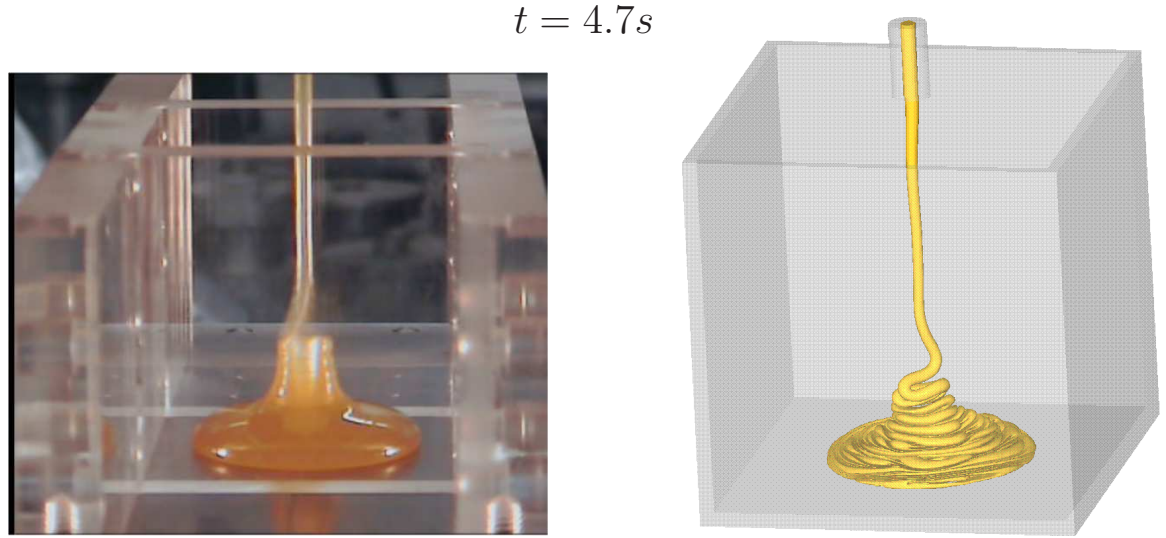

$t=9.4 s$

Figura 8.3: Comparação entre os resultados experimentais e numéricos para o jato oscilante circular obtidos com o esquema PFDPUS nos tempos $t=1.5 \mathrm{~s}, t=2.8 \mathrm{~s}, t=4.7 \mathrm{~s}$ e $t=9.4 \mathrm{~s}$. 
A Figura 8.4 ilustra os resultados obtidos com o esquema PFDPUS em seis tempos $(t=0.3 s$, $t=0.6 s, t=0.9 s, t=1.2 s, t=1.5 \mathrm{~s}$ e $t=1.8 \mathrm{~s})$. A partir das figuras é possível ver que as dobras que surgem no escoamento quando o fluido toca a superfície rígida são bem capturadas, o que mais uma vez confirma que o esquema PFDPUS é apropriado também para simular escoamentos de fluidos altamente viscosos.

\subsection{Ressalto hidráulico circular}

Para finalizar, nesta seção apresenta-se a simulação do ressalto hidráulico circular no caso 3D (já simulado anteriormente no caso axissimétrico). No caso em que o número de Reynolds é suficientemente alto, a forma mais comum do escoamento aparece quando a película de fluido é suave antes do ressalto e instável após ele (ver experimento de Ellegard et al. [19] na Figura 8.5-(a)).

Para a simulação do problema é utilizado o código Freeflow 3D [9] equipado com o esquema PFDPUS proposto. A condição no-slip é adotada na superfície rígida e os seguintes dados para a simulação são:

- Malha: $120 \times 120 \times 10$ células computacionais $(\delta x=\delta y=\delta z=0.005 m)$;

- Dimensão do domínio: $0.6 m \times 0.6 m \times 0.05 m$;

- Diâmetro do injetor: $d=0.05 m$;

- Escala de comprimento: $L_{0}=d=0.05 m$;

- Escala de velocidade: $U_{0}=1 \mathrm{~m} / \mathrm{s}$;

- Coeficiente de viscosidade cinemática: $\nu=5 \times 10^{-5} \mathrm{~m}^{2} / \mathrm{s}$;

- Número de Reynolds: $R e=1000$.

Além disso, o injetor está posicionado a uma distância $H=0.001 \mathrm{~m}$ da superfície rígida. $\mathrm{O}$ resultado numérico obtido em $t=1.8 \mathrm{~s}$ está comparado, na Figura 8.5, com o experimento de Ellegard et al. [19]. Nota-se que o esquema PFDPUS simula com sucesso esse experimento. 


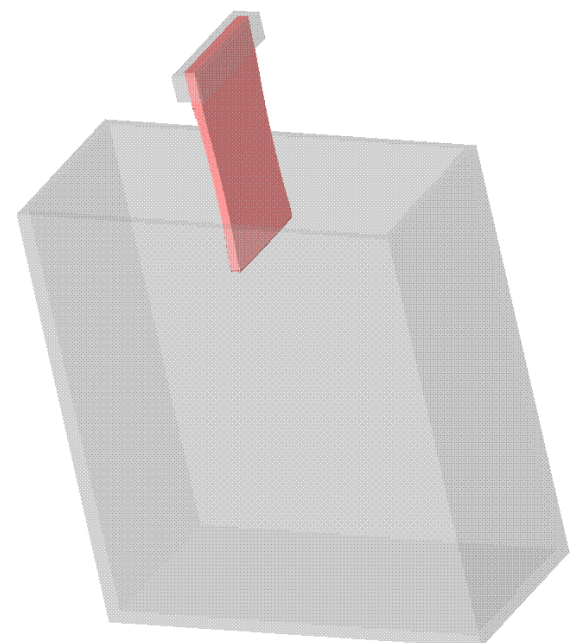

$t=0.3 \mathrm{~s}$

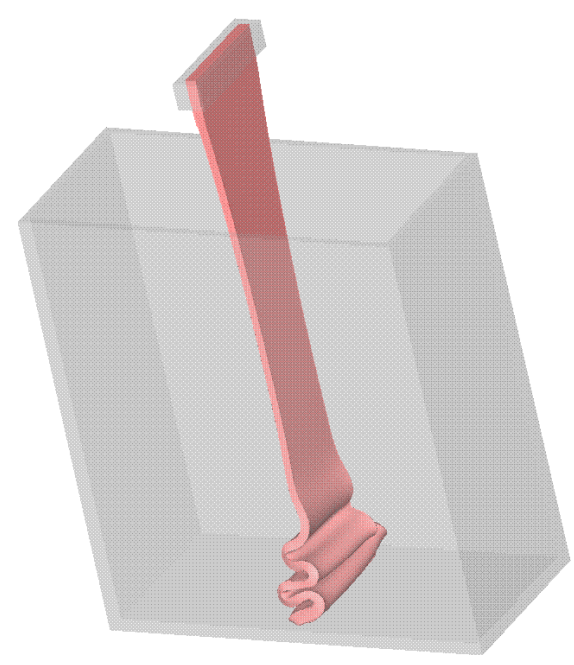

$t=0.9 \mathrm{~s}$

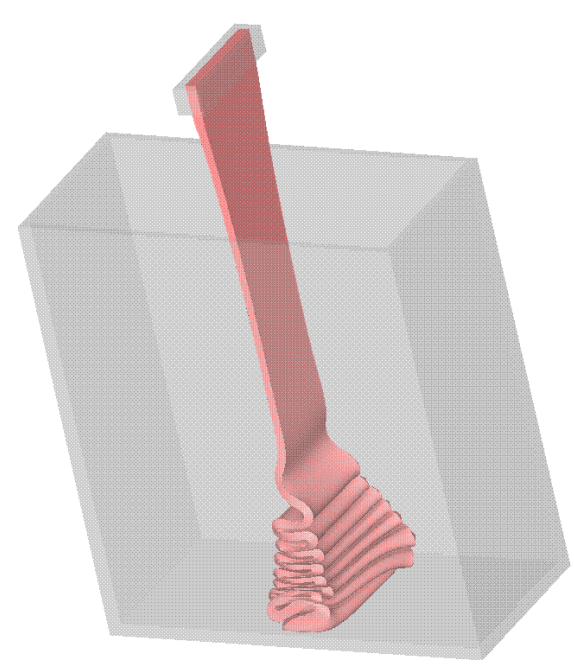

$t=1.5 \mathrm{~s}$

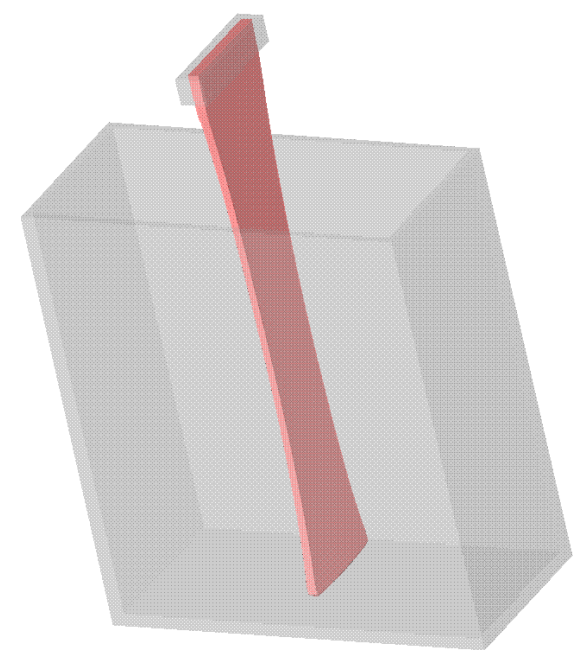

$t=0.6 s$

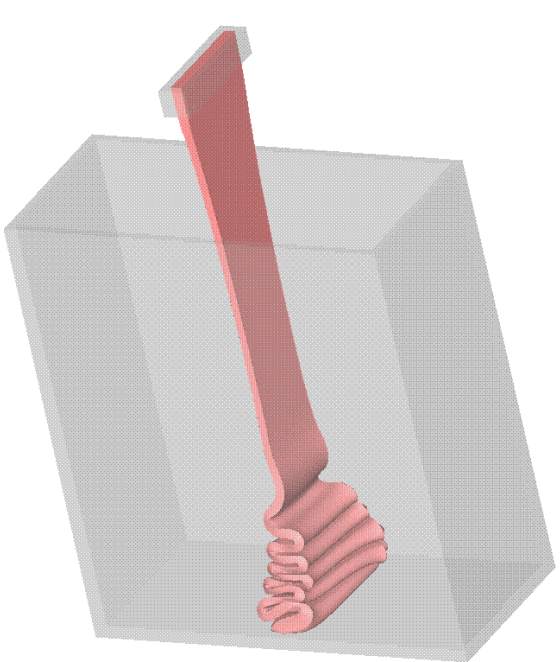

$t=1.2 \mathrm{~s}$

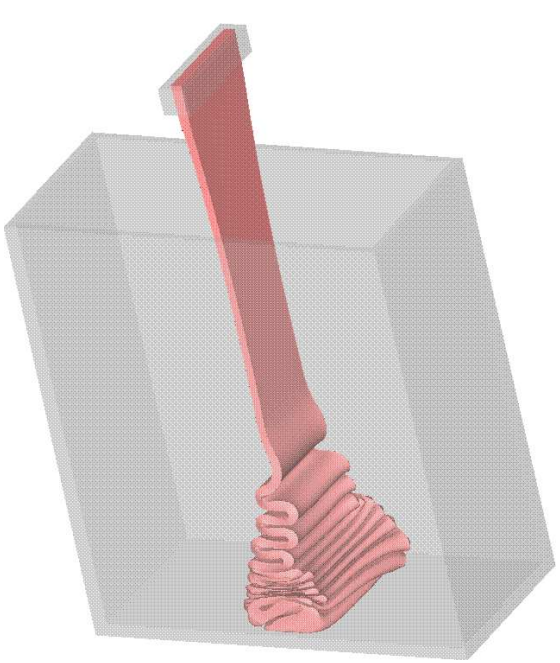

$t=1.8 s$

Figura 8.4: Resultados numéricos para o jato oscilante planar obtidos com o esquema PFDPUS nos tempos $t=0.3 s, t=0.6 s, t=0.9 s, t=1.2 s, t=1.5 \mathrm{~s}$ e $t=1.8 \mathrm{~s}$. 


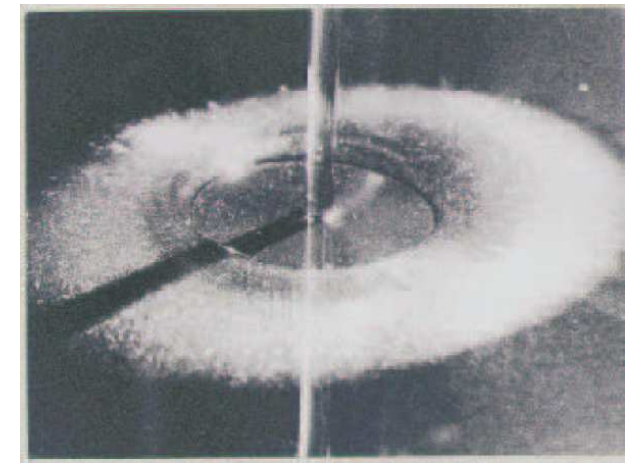

(a) Experimento de Ellegard et al. [19]

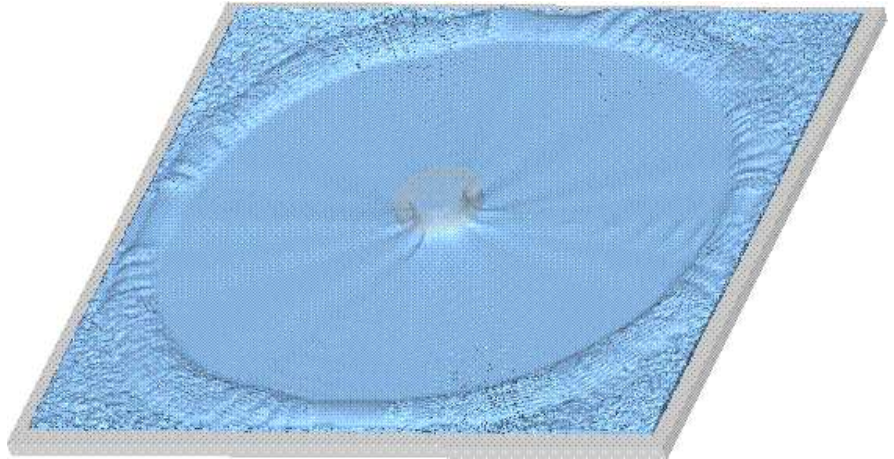

(b) PFDPUS

Figura 8.5: Comparação entre o resultados experimental de Ellegard et al. [19] e o numérico obtido com o esquema PFDPUS para o ressalto hidráulico circular em $t=1.8 \mathrm{~s}$. 


\section{CAPÍTULO 9 \\ Conclusões e trabalhos futuros}

A pesquisa científica descrita nesse texto apresentou o desenvolvimento, análise e implementação de um novo esquema upwind de alta resolução, denominado PFDPUS, para a discretização de termos convectivos de leis de conservação gerais e das equações de Navier-Stokes na formulação incompressível. Esse esquema, uma função polinomial por partes, resulta da aplicação dos critérios de estabilidade TVD/CBC combinados com as quatro condições de Leonard. O esquema PFDPUS é apresentado em variáveis normalizadas de Leonard e na técnica de limitador de fluxo, mostrando possuir duas características importantes: simplicidade e robustez. Várias versões não oscilatórias do esquema para simulação de problemas complexos foram introduzidas impondo os seguintes pares de parâmetros livres: $\alpha=0$ e $\beta=-38 ; \alpha=3$ e $\beta=100 ; \alpha=9$ e $\beta=88 ; \alpha=10$ e $\beta=100$. Com esses pares de parâmetros, o desempenho do novo esquema foi avaliado em uma variedade de problemas de leis de conservação 1D e 2D. Como aplicação, o esquema PFDPUS com o par de parâmetros $\alpha=10$ e $\beta=100$ foi usado para a simulação de escoamentos incompressíveis com superfícies livres móveis 2D, 3D e axissimétricos. As simulações foram feitas usando software CLAWPACK para problemas compressíveis e o ambiente de simulação Freeflow para escoamentos incompressíveis.

De maneira geral, os resultados numéricos 1D apontam que esse novo esquema upwind de alta resolução tem desempenho comparável com os demais esquemas estudados, mostrando ser capaz de controlar oscilações numéricas próximas as descontinuidades e introduzir pouca dissipação numérica. Além disso, a partir das comparações realizadas quanto ao avanço temporal explícito por Euler e Runge-Kutta TVD de terceira ordem, ficou constatado que o desempenho do esquema PFDPUS melhora significativamente quando alta ordem no tempo é empregada. Vale ressaltar que os melhores resultados com o esquema PFDPUS foram alcançados quando o par de parâmetros livres $\alpha=10$ e $\beta=100$ foi utilizado.

Em particular, com o sucesso obtido nas simulações 1D, o esquema PFDPUS foi então 
aplicado na resolução de leis conservação 2D e escoamentos incompressíveis com superfícies livres móveis (para uma ampla faixa do número de Reynolds). Os resultados numéricos foram comparados com dados experimentais, numéricos e analíticos. A partir dos resultados ficou claro que o novo esquema PFDPUS pode ser usado com confiança quando pretende-se simular equações hiperbólicas 2D e escoamentos de fluidos com superfícies livres móveis altamente complexos.

Em síntese, esta pesquisa, além de dispor de um esquema upwind de alta resolução alternativo, fornece também uma variedade de dados numéricos precisos de simulações com esse novo esquema, o que permitirá, no futuro, que outros pesquisadores comparem seus dados com os aqui apresentados.

A continuidade do trabalho pode se dar de muitas maneiras como, por exemplo, analisar o desempenho do esquema PFDPUS com outros parâmetros livres $\alpha$ e $\beta$ que não foram contemplados nesse estudo. Além disso, a combinação do esquema PFDPUS com modelagens da turbulência pode ser aplicada para resolver numericamente uma variedade de problemas de escoamentos incompressíveis com superfícies livres móveis envolvendo: tensão superficial, transferência de calor, fenômenos de convecção/difusão em fluidos não newtonianos e fenômenos interfaciais em escoamentos multifásicos. 
CAPÍTULO 10

\section{Produção científica}

Neste capítulo são listados os artigos publicados/submetidos originados dessa dissertação.

\subsection{Artigos publicados}

- Giseli A. B. Lima, Laís Corrêa, Miguel A. C. Candezano, Patrícia Sartori e Valdemir G. Ferreira, A simple NVD/TVD-based upwinding scheme for convection term discretization, ECCOMAS CFD 2010-Fifth European Conference on Computational Fluid Dynamics, 2010, Lisboa/Portugal.

Abstract: The correct modeling for processes involving convection, without introducing excessive artificial damping while retaining high accuracy, stability, boundedness and simplicity of implementation continues being nowadays a challenging task for the cientific CFD community (see [1,2,5]). In this context, the objective of this study is to present and to evaluate the performance of a new TVD-based upwinding scheme, namely Six-Degree Polynomial Upwind Scheme of C1 Class (SDPUS-C1), for convection term discretization. SDPUS-C1 satisfies the TVD principle of Harten [3] and is based on the NVD formulation of Leonard [4]. Firstly, a description of the scheme is done and then numerical results are presented for three-dimensional hyperbolic conservation laws, such as acoustics, Burgers and Euler equations. Finally, as application, the SDPUS-C1 scheme is used for the computational simulation of three-dimensional incompressible fluid flows involving moving free surfaces.

- Patrícia Sartori, Giseli A. B. Lima, Laís Corrêa, Miguel A. C. Candezano e Valdemir G. Ferreira, Avaliação computacional de três esquemas upwind originais, SIMMEC 2010 - $9^{\circ}$ Simpósio de Mecânica Computacional, 2010, São João Del Rei/MG.

Resumo: Resolver numericamente problemas em dinâmica dos fluidos é uma tarefa difícil e desafiadora, principalmente quando tais problemas são dominados por convecção. Isso requer 
o desenvolvimento de esquemas numéricos tipo upwind que sejam precisos, monotônicos e robustos. O presente trabalho é destinado à avaliação computacional de três novos esquemas upwind de alta resolução, desenvolvidos no LCAD-ICMC/USP, denominados ADBQUICKEST (ADaptative Quickest), TOPUS (Third-Order Polynomial Upwind Scheme) e SDPUS-C1 (Six-Degree Polynomial Upwind Scheme of C1 Class). O desempenho desses esquemas é investigado a partir da simulação computacional de problemas de leis de conservação hiperbólicas, a saber, advecção e problemas de Riemann para acústica e Euler da dinâmica dos gases. E então, como aplicação, esses esquemas são utilizados na simulação de escoamentos incompressíveis com superfícies livres móveis modelados pelas equações de Navier-Stokes 3D.

- Laís Corrêa, Giseli A. B. Lima, Patrícia Sartori, Miguel A. C. Candezano e Valdemir G. Ferreira, A new polynomial upwind convection scheme for fluid flow simulations, CONEM 2010 - VI Congresso Nacional de Engenharia Mecânica, 2010, Campina Grande/PB.

Abstract: The simulation of fluid flow problems involving strong convective character is a difficult problem to solve and has atracted many researchers in the CFD community. In this scenario, we present in this work a new polynomial upwind scheme, called SDPUS ("Six-Degree Polynomial Upwind Scheme of C1 Class"), for numerical solution of conservation laws and related fluid dynamics problems. The scheme is developed in the context of normalized variables of Leonard and satisfies the CBC and TVD stability criteria of Gaskell and Lau, and Harten, respectively. The numerical solutions obtained with this scheme can achieve second/third order of accuracy in smooth regions and first order near to discontinuities (shocks). The performance of the SDPUS is assessed in the solution of nonlinear hyperbolic systems, such as shallow water, acoustics, and Euler equations of gas dynamics. As application, the scheme is then used in the solution of incompressible Navier-Stokes equations in cylindrical coordinates. From numerical results, one can clearly see that the SDPUS-C1 scheme is a robust tool for resolving both compressible and incompressible complex flow problems.

- Miguel A. C. Candezano, Patrícia Sartori e Valdemir G. Ferreira, An upwind implementation of some conservation laws, DINCON'10 - 9th Brazilian Conference on Dynamics, Control and their Applications, 2010, Serra Negra/SP.

Abstract: This work provides a computational assessment of three high-resolution upwind schemes namely ADBQUICKEST, TOPUS and CUBISTA for solving some fluid dynamics problems. These schemes are developed in the context of the normalized variables (NV) of Leonard and in TVD constraints of Harten, and implemented using the finite difference methodology. We consider the application of these schemes to systems of conservation laws, $1 D$ and 2D, to demonstrate their performance in modeling such problems.

- Miguel A. C. Candezano, Patrícia Sartori, Laís Corrêa, Giseli A. B. Lima e Valdemir G. Ferreira, A computational evaluation of two high-resolution convective schemes for problems in 
fluid dynamics, ENCIT 2010 - XIII Brazilian Congress of Thermal Sciences and Engineering, 2010, Uberlândia/MG.

Abstract: It is well recognized that researchers face many problems for numerically approximating nonlinear convective terms in conservation laws and related fluid dynamics problems. One of the main challenges is to develop upwind schemes that capture well discontinuities (or shock waves) and allow high (at least second order) accuracy solution. In this scenario, the goal of this work is to present a computational evaluation of two genuinely Brazilian high resolution convective upwind schemes, namely ADBQUICKEST and SDPUS-C1, for solving general fluid dynamics problems. Both schemes are developed in the context of normalized variables (NV) of Leonard and satisfy the total-variation diminishing (TVD) constraints of Harten.

- Patrícia Sartori, Miguel A. C. Candezano e Valdemir G. Ferreira, Avaliação de dois esquemas para a captura de choque para leis de conservação nas formas conservativa e não-conservativa, CNMAC 2010 - XXXIII Congresso Nacional de Matemática e Aplicada e Computacional, 2010, Águas de Lindóia/SP.

Resumo: Nos últimos anos, novos esquemas upwind para captura de choque vem sendo desenvolvidos para aproximar termos convectivos (em geral não lineares) de leis de conservação; como exemplos pode-se citar os esquemas ADBQUICKEST (ADaptative Bounded QUICKEST) [1] e TOPUS (Third-Order Polynomial Upwind Scheme) [2], originalmente desenvolvidos no LCAD-ICMC-USP. Objetiva-se com esses esquemas resolver bem descontinuidades (ou choques) usando uma forma adequada da equao modelo. Para tanto, prope-se neste trabalho mostrar a importância da modelagem numérica na forma conservativa e constatar, por meio de exemplos numéricos, que a forma não-conservativa pode levar a resultados que parecem razoáveis no choque mas são, na maioria das vezes, incorretos. Isso pode ocorrer pois a forma não-conservativa pode fornecer o cálculo incorreto das velocidades nas vizinhanças das descontinuidades.

\subsection{Artigos submetidos}

- Miguel A. C. Candezano, Patrícia Sartori, Laís Corrêa, Giseli A. B. Lima, Rodolfo Perez e Valdemir G. Ferreira, A numerical study of some recently introduced TVD upwinding schemes with applications in fluid dynamics problems, COBEM 2011 - 21st International Congress of Mechanical Engineering, 2011, Natal/RN.

Abstract: An important issue in computational fluid dynamics is the appropriate approximation of the convection phe nomena. For this, the TVD schemes are alternatives to the ENO/WENO techniques due to the robustness, low cost and simplicity of implementation. Within this scenario, the aim of the our work is to present a numerical study of some recently introduced polynomial TVD upwinding schemes - namely TOPUS and SDPUS - with appli- 
cations in fluid dynamics problems. By using these new upwind schemes, numerical results for nonconvex nonlinear problem, $2 D$ advection of scalars and $2 D$ Euler equations are presented. Comparison with the well recognized CFL-dependent ARORA-ROE and ADBQUICKEST schemes and the conventional SUPERBEE and MC schemes are assessed. The TOPUS and SDPUS upwind schemes are developed in the context of normalized variables (NV) of Leonard and satisfy TVD constraints of Harten. 


\section{O esquema PFDPUS e os parâmetros livres $\alpha$ e $\beta$}

Este apêndice apresenta uma breve descrição de como são determinados os parâmetros livres $\alpha$ e $\beta$ do esquema PFDPUS que o tornam TVD. Para isso, considera-se o esquema PFDPUS em variáveis normalizadas da forma

$$
\hat{\phi}_{f}= \begin{cases}(-14+4 \alpha) \hat{\phi}_{U}^{4}+(9-4 \alpha) \hat{\phi}_{U}^{3}+\alpha \hat{\phi}_{U}^{2}+\hat{\phi}_{U}, & \text { se } \hat{\phi}_{U} \in\left[0, \frac{1}{2}\right), \\ \left(2+\frac{4}{13} \beta\right) \hat{\phi}_{U}^{4}+\left(-3-\frac{12}{13} \beta\right) \hat{\phi}_{U}^{3}+\beta \hat{\phi}_{U}^{2}+\left(2-\frac{6}{13} \beta\right) \hat{\phi}_{U}+\left(\frac{1}{13} \beta\right), & \text { se } \quad \hat{\phi}_{U} \in\left[\frac{1}{2}, 1\right], \\ \hat{\phi}_{U}, & \text { se } \quad \hat{\phi}_{U} \notin[0,1],\end{cases}
$$

e as restrições TVD de Harten [26] definidas por (ver Figura 3.4)

$$
\left\{\begin{array}{lll}
\hat{\phi}_{f} \in\left[\hat{\phi}_{U}, 2 \hat{\phi}_{U}\right] \quad \text { e } \quad \hat{\phi}_{f} \leq 1, & \text { se } \quad \hat{\phi}_{U} \in[0,1] \\
\hat{\phi}_{f}=\hat{\phi}_{U}, & \text { se } & \hat{\phi}_{U} \notin[0,1] .
\end{array}\right.
$$

Para que o esquema PFDPUS seja TVD, constata-se a partir de (A.2) que as seguintes condições devem ser satisfeitas:

i) $\hat{\phi}_{f} \geq \hat{\phi}_{U}$ para $0 \leq \hat{\phi}_{U}<\frac{1}{2}$.

Seja $h=\hat{\phi}_{f}-\hat{\phi}_{U}$. Substituindo a primeira sentença de (A.1) em $h$, obtém-se

$$
h=(-14+4 \alpha) \hat{\phi}_{U}^{4}+(9-4 \alpha) \hat{\phi}_{U}^{3}+\alpha \hat{\phi}_{U}^{2}
$$

Assim, $h \geq 0$ para $0<\hat{\phi}_{U} \leq \frac{1}{2}$.

ii) $\hat{\phi}_{f} \leq 2 \hat{\phi}_{U}$ para $0 \leq \hat{\phi}_{U}<\frac{1}{2}$. 
Considere $g=\hat{\phi}_{f}-2 \hat{\phi}_{U}$. Substituindo a primeira sentença de (A.1) em $g$, obtém-se

$$
g=(-14+4 \alpha) \hat{\phi}_{U}^{4}+(9-4 \alpha) \hat{\phi}_{U}^{3}+\alpha \hat{\phi}_{U}^{2}-\phi_{U}
$$

Logo, $g \leq 0$ para $0 \leq \hat{\phi}_{U}<\frac{1}{2}$.

iii) $0<\hat{\phi}_{f} \leq 1$ para $0 \leq \hat{\phi}_{U}<\frac{1}{2}$.

Seja $s=\hat{\phi}_{f}-\frac{1}{2}$. Substituindo a primeira sentença de (A.1) em $s$, obtém-se

$$
s=(-14+4 \alpha) \hat{\phi}_{U}^{4}+(9-4 \alpha) \hat{\phi}_{U}^{3}+\alpha \hat{\phi}_{U}^{2}+\phi_{U}-1
$$

Assim, $s \leq 0$ para $0 \leq \hat{\phi}_{U}<\frac{1}{2}$.

iv) $\hat{\phi}_{f} \geq \hat{\phi}_{U}$ para $\frac{1}{2} \leq \hat{\phi}_{U} \leq 1$.

Seja $p=\hat{\phi}_{f}-\hat{\phi}_{U}$. Substituindo a segunda sentença de (A.1) em $p$, segue

$$
p=\left(2+\frac{4}{13} \beta\right) \hat{\phi}_{U}^{4}+\left(-3-\frac{12}{13} \beta\right) \hat{\phi}_{U}^{3}+\beta \hat{\phi}_{U}^{2}+\left(1-\frac{6}{13} \beta\right) \hat{\phi}_{U}+\left(\frac{1}{13} \beta\right) .
$$

Dessa forma, $p \geq 0$ para $\frac{1}{2} \leq \hat{\phi}_{U} \leq 1$.

v) $\frac{1}{2} \leq \hat{\phi}_{f}<1$ para $\frac{1}{2} \leq \hat{\phi}_{U} \leq 1$.

Considera-se agora $r_{1}=\hat{\phi}_{f}-\frac{1}{2}$ e $r_{2}=\hat{\phi}_{f}-1$. Substituindo a segunda sentença de (A.1) em $r_{1}$ e $r_{2}$, segue

$$
\begin{aligned}
& r_{1}=\left(2+\frac{4}{13} \beta\right) \hat{\phi}_{U}^{4}+\left(-3-\frac{12}{13} \beta\right) \hat{\phi}_{U}^{3}+\beta \hat{\phi}_{U}^{2}+\left(1-\frac{6}{13} \beta\right) \hat{\phi}_{U}+\left(\frac{1}{13} \beta-\frac{1}{2}\right), \\
& r_{2}=\left(2+\frac{4}{13} \beta\right) \hat{\phi}_{U}^{4}+\left(-3-\frac{12}{13} \beta\right) \hat{\phi}_{U}^{3}+\beta \hat{\phi}_{U}^{2}+\left(1-\frac{6}{13} \beta\right) \hat{\phi}_{U}+\left(\frac{1}{13} \beta-1\right) .
\end{aligned}
$$

Dessa forma, deve-se ter $r_{1} \geq 0$ e $r_{2} \leq 0$ para $\frac{1}{2}<\hat{\phi}_{U} \leq 1$.

Para determinar os parâmetros livres $\alpha$ e $\beta$ que satisfaçam todas as condições acima, um programa escrito na linguagem $\mathrm{C}$, que varre valores inteiros para $\alpha$ e $\beta$, é utilizado. Os resultados obtidos mostram que para $\alpha \in[0,10]$ o esquema é TVD para $\hat{\phi}_{U} \in\left[0, \frac{1}{2}\right)$. Já para $\beta$, os resultados apresentam inúmeros valores entre -38 e 100. Dentre eles, $\beta=-38, \beta=88$ e $\beta=100$ são alguns dos valores que tornam o esquema PFDPUS TVD para $\hat{\phi}_{U} \in\left[\frac{1}{2}, 1\right]$.

Em resumo, as combinações entre os parâmetros livres $\alpha$ e $\beta$ que tornam o esquema PFDPUS TVD e que são utilizados nesse trabalho são: $\alpha=0$ e $\beta=-38, \alpha=3$ e $\beta=100, \alpha=9$ e $\beta=88$, e, $\alpha=10$ e $\beta=100$. A Figura 4.1 do Capítulo 4 mostra, respectivamente, o esquema PFDPUS nos planos $\hat{\phi}_{f} \perp \hat{\phi}_{U}$ e $\psi \perp r$ para as quatro combinações escolhidas para $\alpha$ e $\beta$. Como pode-se notar, este esquema está inteiramente contido na região TVD. 


\section{APÊNDICE $\mathcal{B}$}

\section{CLAWPACK}

Este apêndice apresenta uma breve descrição do pacote computacional CLAWPACK (Conservation LAW PACKage). Esse pacote computacional foi desenvolvido por Leveque et al. [44] e, no contexto do método de volumes finitos, simula leis de conservação hiperbólicas 1D, 2D e 3D. Como visto no Capítulo 2, as leis de conservação 1D podem ser escritas da forma

$$
\phi_{t}+F(\phi)_{x}=0
$$

onde $\phi$ é chamado de vetor das variáveis conservadas e $F=F(\phi)$ é o vetor da função fluxo. Em uma dimensão, para a solução de (B.1) é empregado a forma quasi-linear das equações

$$
\phi_{t}+A(x, t) \phi_{x}=0
$$

em que $A(x, t)$ é a matriz jacobiana $F^{\prime}(\phi(x, t))=A(x, t)$.

Para a resolução das leis de conservação 1D é empregado o algoritmo:

Passo 1: Substitui-se a solução pontual por uma distribuição constante por partes;

Passo 2: Resolve-se o problema de Riemann aproximado pelo método de Roe (ver detalhes em Leveque [43] e Toro [72]). O solucionador do problema de Riemann retorna, para quaisquer dois estados $\phi_{i-1}$ e $\phi_{i}$, um conjunto de $M$ ondas definidas como

$$
\sum_{q=1}^{M} \mathcal{W}_{i-\frac{1}{2}}^{q}=\phi_{i}-\phi_{i-1}=\Delta \phi_{i-\frac{1}{2}}
$$

e as flutuações

$$
\mathcal{A}^{-} \Delta \phi_{i-\frac{1}{2}}=\sum_{q}^{M}\left(\hat{s}^{q}\right)^{-} \mathcal{W}_{i-\frac{1}{2}}^{q}
$$




$$
\mathcal{A}^{+} \Delta \phi_{i-\frac{1}{2}}=\sum_{q}^{M}\left(\hat{s}^{q}\right)^{+} \mathcal{W}_{i-\frac{1}{2}}^{q}
$$

onde $\left(\hat{s}^{q}\right)^{-}=\min (\hat{\lambda}, 0)$ e $\left(\hat{s}^{q}\right)^{+}=\max (\hat{\lambda}, 0)$, em que $\hat{\lambda}$ é o outovalor da matriz $A$.

Passo 3: Aplica-se a fórmula de atualização. As soluções são computadas usando método de primeira ordem de Godunov ou por sua variante de segunda ordem de precisão proposto por LeVeque [43], o método de primeira ordem de Godunov com termo de correção:

- Método de primeira ordem de Godunov:

$$
\phi_{i}^{n+1}=\phi_{i}^{n}-\frac{\delta t}{\delta x}\left(\mathcal{A}^{+} \Delta \phi_{i-\frac{1}{2}}+\mathcal{A}^{-} \Delta \phi_{i+\frac{1}{2}}\right)
$$

- Método de primeira ordem de Godunov com termo de correção:

$$
\phi_{i}^{n+1}=\phi_{i}^{n}-\frac{\delta t}{\delta x}\left(\mathcal{A}^{+} \Delta \phi_{i-\frac{1}{2}}+\mathcal{A}^{-} \Delta \phi_{i+\frac{1}{2}}\right)-\frac{\delta t}{\delta x}\left(\tilde{F}_{i+\frac{1}{2}}-\tilde{F}_{i-\frac{1}{2}}\right),
$$

onde

$$
\tilde{F}_{i-\frac{1}{2}}=\mathcal{A}^{+} \phi_{i}+\mathcal{A}^{-} \phi_{i-1}+\frac{1}{2}|A|\left(I-\frac{\delta t}{\delta x}|A|\right)\left(\phi_{i}-\phi_{i-1}\right),
$$

com

$$
\phi_{i}-\phi_{i-1}=\sum_{q}^{M} \tilde{\alpha}_{i-\frac{1}{2}} \hat{s}^{q} \quad \text { e } \quad \tilde{\alpha}_{i-\frac{1}{2}}=\alpha_{i-\frac{1}{2}} \psi\left(r_{i-\frac{1}{2}}^{q}\right),
$$

em que $\alpha=R^{-1} \delta, R$ é a matriz dos autovetores, $\delta=\phi_{i}-\phi_{i-1}, \psi$ são as funções limitadores de fluxo e $r_{1-\frac{1}{2}}^{q}$ é a razão dos gradientes consecutivos definida por

$$
r_{i-\frac{1}{2}}^{q}=\frac{\alpha_{I-\frac{1}{2}}^{q}}{\alpha_{i-\frac{1}{2}}^{q}} \quad \text { em que } \quad I= \begin{cases}i-1, & \text { se } \lambda^{q} \leq 0 \\ i+1, & \text { se } \quad \lambda^{q}>0\end{cases}
$$

$\operatorname{com} \lambda^{q}$ autovalores.

Vale ressaltar, que a partir do método de primeira ordem de Godunov com termo de correção dado por (B.7), é possível implementar no CLAWPACK uma variedade de métodos numéricos a partir de pequenas modificações nas rotinas que implementam os limitadores de fluxo. Essas modificações tem o objetivo de acrescentar os limitadores fluxos de outros esquemas desejados. 


\section{Referências Bibliográficas}

[1] M. A. Alves, P. J. Oliveira, and F. T. Pinho. A convergent and universally bounded interpolation scheme for the treatment of advection. International Journal for Numerical Methods in Fluids, 41:47-75, 2003.

[2] S. W. Armfield. Finite difference solutions of the Navier-Stokes equations on staggered and non-staggered grids. Computers \& Fluids, 20:1-17, 1991.

[3] J. Balbás, E. Tadmor, and Cheng-Chin Wu. Non-oscillatory central schemes for one- and two-dimensional MHD equations: I. Journal of Computational Physics, 201:261-285, 2004.

[4] D. S. Balsara and C. W. Shu. Monotonicity preserving weighted essentially non-oscilatory scheme with increasingly high order of accuracy. Journal of Computational Physics, 160:405-452, 2000.

[5] J. Boussinesq. Memoires presentes par divers savants sciences mathematique at physiques theorie de l'ecoulente tourbillant. Master's thesis, Academie des Scienses, 1877.

[6] A. C. Brandi. Estratégias upwind e modelagem $\kappa-\varepsilon$ para simulação numérica de escoamentos com superfícies livres em altos números de Reynolds. Master's thesis, Instituto de Ciências Matemáticas e de Computação (ICMC-USP), 2005.

[7] M. Brio and C. C. Wu. An upwind differencing scheme for the equations of ideal magnetohydrodynamics. Journal of Computaional Physics, 75:400-422, 1988.

[8] M. Cada and M. Torrilhon. Compact third-order limiter functions for finite volume methods. Journal of Computational Physics, 228:4118-4145, 2009.

[9] A. F. Castelo, M. F. Tomé, C. N. L. César, S. McKee, and J. A. Culminato. Freeflow: An integrated simulation system for three-dimensional free-surface flows. Journal of Computers and Visualization in Science, 2:199-210, 2000. 
[10] A. J. Chorin. Numerical solution of the Navier-Stokes equations. Mathematics Computational, 22:745-762, 1968.

[11] A. Colagrossi and M. Landrini. Numerical simulation of interfacial flows by smoothed particle hydrodynamics. Journal Computation Physics, 191:448-475, 2003.

[12] G. Colicchio, M. Landrini, and L. C. Chaplin. Level-set modelling of the air-water flow generated buy a surface piercing body. In Proc 8th Int. Conference on Numerical Ship Hydrodynamics, Korea, 2003.

[13] J. O. Cruickshank. Low-Reynolds-number instabilities in stagnating jet flows. Journal of Fluid Mechanics, 193:111-127, 1987.

[14] C. Dawson and J. Proft. Discontinuous and coupled continuous/discontinuous Galerkin methods for the shallow water equations. Computer Methods in Applied Mechanics and Engineering, 191:4721-4746, 2002.

[15] G. A. B. de Lima. Desenvolvimento de estratégias de captura de descontinuidades para leis de conservação e problemas relacionados em dinâmica dos fluidos. Master's thesis, Instituto de Ciências Matemáticas e de Computação (ICMC-USP), 2010.

[16] Maria Luísa Bambozzi de Oliveira. FREEFLOW-AXI: Um ambiente de simulação de escoamentos axissimétricos com superfícies livres. Master's thesis, Instituto de Ciências Matemáticas e de Computação (ICMC-USP), 2002.

[17] F. M. Denaro. On the applications of the helmoltz-hodge decomposition in projection methods for incompressible flows with general boundary conditions. International Journal for Numerical Methods in Fluids, 43:43-69, 2003.

[18] P. A. Durbin. On the $\kappa-\varepsilon$ stagnation point anomaly. International Journal of Heat and Fluid Flow, 17:89-90, 1996.

[19] C. Ellegaard, A. E. Hansen, A. Haaning, K. Hansen, A. Marcussen, and T. Bohr. Poligonal hydraulic jumps. Nonlinearity, 12:1-7, 1999.

[20] V. G. Ferreira, F. A. Kurokawa, R. A. B. Queiroz, M. K. Kaibara, C. M. Oishi, J. A. Cuminato, A. Castelo, M. F. Tomé, and S. McKee. Assessment of a high-order finite difference upwind scheme for the simulation of convection-diffusion problems. International Journal for Numerical Methods in Fluids, 60:1-26, 2009.

[21] A. O. Fortuna. Técnicas computacionais para a dinâmica dos fluidos: conceitos básicos e aplicações. EDUSP, 2000. 
[22] P. H. Gaskell and A. K. C. Lau. Curvature-compensated convective transport: SMART, a new boundedness preserving transport algorithm. International Journal for Numerical Methods in Fluids, 8:617-641, 1988.

[23] S. Gottlieb. On high-order strong stability preserving Runge-Kutta and multi step time discretizations. Journal of Scientific Computing and Visualization in Science, 25:105-128, 2005.

[24] S. Gottlieb and C. W. Shu. Total variation diminishing Runge-Kutta schemes. Mathematics of Computation, 67:73-85, 1998.

[25] M. Greco, M. Landrini, and O. M. Faltinsen. Impact flows and loads on shi-deck structures. Journal Fluids Structures, page submitted, 2003.

[26] A. Harten. High resolution schemes for hyperbolic conservation laws. Journal of Computational Physics, 49:357-393, 1983.

[27] A. Harten. ENO scheme with subcell resolution. Journal of Computational Physics, 83:148-184, 1989.

[28] M. R. Hestenes and E. Stiefel. Methods of conjugate gradients for solving linear systems. Journal of Research of the National Bureau of Standards, 49:409-436, 1952.

[29] C. Hirsch. Numerical Computation of Internal and External Flows, Volume 1, Second Edition: The Fundamentals of Computational Fluid Dynamics. Butterworth-Heinemann. ELSEVIER, 2007.

[30] G. S. Jiang and C. W. Shu. Efficient implementation of weighted ENO schemes. Journal of Computational Physics, 126:202-228, 1996.

[31] G. S. Jiang and C. C Wu. A high-order WENO finite difference scheme for the equations of ideal magnetohydrodynamics. Journal of Computational Physics, 150:561-594, 1999.

[32] F. Kemm. A comparative study of TVD-limiters-well-known limiters and an introduction of new ones. International Journal for Numerical Methods in Fluids, 2010.

[33] S. Koshizuka and S. Oka. Moving-particle semi-implicit method for fragmentation of incompressible fluids. Nuclear Science and Engineering, 123:421-434, 1996.

[34] K. B. Kuan and C. A. Lin. Adaptive QUICK-based scheme to approximate convective transport. AIAA Journal, 38:2233-2237, 2000.

[35] V. Kumar and S. V. R. Rao. Composite scheme using localized relaxation with non-standard finite difference methods for hyperbolic conservation laws. Journal of Sound and Vibration, 311:786-801, 2008. 
[36] A. Kurganov and E. Tadmor. Solution of two-dimensional Riemann problems for gas dynamics without Riemann problem solvers. Technical report, CAM 0034 UCLA Los Angeles, 2000.

[37] M. Kurihara. A consideration about hydraulic jump. Technical report, Kyusyu Imperial University, 1946.

[38] F. A. Kurokawa. Um esquema upwind para leis de conservação e sua aplicação na simulação de escoamentos incompressíveis 2D e 3D laminares e turbulentos com superfícies livres. Master's thesis, Instituto de Ciências Matemáticas e de Computação (ICMC-USP), 2009.

[39] B. Van Leer. Towards the ultimate conservative difference scheme. IV. A new approach to numerical convection. Journal of Computational Physics, 23:276-299, 1977.

[40] B. P. Leonard. The QUICK algorithm: a uniformly third-order finite difference method for highly convective flows. Computational Methods Applicate Mechanic Engineering, 19:59-98, 1979.

[41] B. P. Leonard. Simple high-accuracy resolution program for convective modelling of discontinuities. International Journal for Numerical Methods in Fluids, 8:1291-1318, 1988.

[42] R. J. LeVeque. Wave propagation algorithms for multidimensional hyperbolic systems. Journal Computational Physics, 131:327-353, 1997.

[43] R. J. LeVeque. Finite volumes methods for hyperbolic problems. Press Syndicate of the University of Cambridge, 2004.

[44] R. J. LeVeque, J. O. Langhset, M. Berger, D. McQueen, D. Calhoun, P. Blossey, and S. Mitram. Clawpack 5.0: software package designed to compute numerical solution to hyperbolic partial differential equations using a wave propagation approach. http://www.amath.washington.edu/claw, 2006.

[45] R. J. LeVeque, D. Mihalas, E. A. Dorfi, and E. Muller. Computational Methods for Astrophysical Fluid Flow. Springer-Verlag, 1998.

[46] C. H. Lin and C. A. Lin. Simple high-order bounded convection scheme to model discontinuities. AIAA Journal, 35:563-565, 1997.

[47] H. Lin and C. C. Chieng. Characteristic-based flux limiters of an essentially third-order flux-splitting method for hyperbolic conservation laws. International Journal for Numerical Methods in Fluids, 13:287-307, 1991. 
[48] J. C. Martin and W. J. Moyce. An experimental study of the collapse of liquid columns on a rigid horizontal plane. Philosophical Transactions of the Royal Society, Mathematical, Phisical \& Engeneering Sciences, 244:312-324, 1952.

[49] S. Middleman. Modeling Axisymmetric Flows: Dynamics of Films, Jets, and Drops. Academic Press, 1995.

[50] K. W. Morton and D. F. Mayers. Numerical Solution of Partial Differential Equations. Cambridge University Press, 2005.

[51] J. M. Nóbrega, O. S. Carneiro, F. T. Pinho, G. S. Paulo, M. F. Tomé, A. castelo, and J. A. Cuminato. The phenomenon of jet buckling: Experimental results and numerical predictions. In The Polymer Processing Society 23rd Anual Meeting.

[52] G. W. Platzman. An exact integral of complet spectral equation for unsteady one-dimensional flow. Master's thesis, University of Chicago, 1964.

[53] R. A. B. Queiroz and V. G. Ferreira. Development and testing of high-resolution upwind schemes - Upwind schemes for incompressible free surface flows. VDM Verlag Dr Müller, 2010.

[54] E. Isaacson R. Courant and M. Rees. On the solution of nonlinear hyperbolic differential equations by finites differentes. Communnity Pure Apllied Mathematics, 5:243-255, 1952.

[55] M. Ricchiuto, Á. Csík, and H. Deconinck. Residual distribution for general time-dependent conservation laws. Journal Computational Physics, 209:249-289, 2005.

[56] A. Ritter. Die fortpflanzung der wassserwellen. Zeitschrift Verein Deutscher Ingenieure, 36:947-954, 1982.

[57] P. L. Roe. Some contribuitions to the modeling of discontinuous flows. Lect. Notes Appl. Math., 22:163-193, 1985.

[58] C. W. Schulz-Rinne. Classification of the Riemann problem for two-dimensional gas dynamics. SIAM Journal on Mathematical Analysis, 24:76â88., 1993.

[59] C. W. Schulz-Rinne, J. P. Collins, and H. M. Glaz. Numerical solution of the Riemann problem for two-dimensional gas dynamics. SIAM Journal on Scientific Computing, 14:1394-1414, 1993.

[60] G. Sod. A survey of several finite difference methods for systems of nonlinear hyperbolic conservation laws. Journal of Computational Physics, 27:1-37, 1978.

[61] D. L. Sondak and R. H. Pletcher. Application of wall functions to generalized nonorthogonal curvilinear systems. AIAA Journal, 33:33-41, 1995. 
[62] B. Song, G. R. Liu, K. Y. Lam, and R. S. Amano. On a higher-order bounded discretization scheme. International Journal for Numerical Methods in Fluids, 32:881-897, 2000.

[63] D. B. Spalding. A novel finite difference formulation for differential expressions involving both first and second derivatives. International Journal for Numerical Methods in Fluids, 4:551-559, 1972.

[64] P. K. Sweby. High resolution schemes using flux limiters for hyperbolic conservation laws. SIAM Journal on Numerical Analysis, 21:995-1011, 1884.

[65] I. Tani. Flow separation in thin liquid layers. Journal of the Physics Society of Japan, $4: 212-215,1948$.

[66] G. I. Taylor. Low Reynolds number flows. National Committee for Fluid Mechanics Films - Illustrated experiments in fluid mechanics, 1974.

[67] Z. Teng. Exact boundary conditions for the initial value problem of convex conservation laws. Journal of Computaional Physics, 229:3792-3801, 2010.

[68] J. W. Thomas. Numerical partial differential equations: finite difference methods. Springer-Verlag, 1995.

[69] M. F. Tomé and S. McKee. GENSMAC: A computational marker-and-cell method for free surface flows in general domains. Journal of Computational Physics, 110:171-186, 1994.

[70] M. F. Tomé and S. McKee. Numerical simulation of viscous flow: buckling of planar jets. International Journal for Numerical Methods in Fluids, 29:705-718, 1999.

[71] E. Toro. Shock-capturing methods for free-surface shallow flows. Wiley, 2001.

[72] E. F. Toro. Rieman solvers and numerical methods for fluids dynamics. Springer-Verlag, 1999.

[73] G. D. van Albada, B. van Leer, and W. W. Roberts. A comparative study of computational methods in cosmic gas dynamics. Astronomy \& Astrophysics, 108:76-84, 1982.

[74] B. VanLeer. Towards the ultimate conservative difference scheme. V. A second-order sequel to Godunov's method. Journal of Computational Physics, 33:101-136, 1977.

[75] A. Varonos and G. Bergeles. Development and assessment of a variable-order non-oscilatory scheme for convection term discretization. International Journal for Numerical Methods in Fluids, 26:1-16, 1988.

[76] R. F. Warming and R. M. Beam. Upwind second-order difference schemes and application in aerodynamics flows. AIAA Journal, 14:1241-1249, 1976. 
[77] N. P. Waterson and H. Deconinck. Design principles for bounded higher-order convection schemes - a unified approach. Journal of Computational Physics, 224:182-207, 2007.

[78] E. J. Watson. The radial spread of a liquid jet over a horizontal plane. Journal of Fluids Mechanics, 20:481-499, 1964.

[79] P. Woodward and P. Colella. The numerical simulation of two-dimensional fluid flow with strong shocks. Journal of Computaional Physics, 54:115-173, 1984.

[80] S. T. Zalesak. A preliminary comparison of modern shock-capturing schemes: linear advection. In Advances in computer methods for partial differential equation VI. Vichnevetsky R. and Stepleman R. S(eds) IMACS: New Brunswick, NJ., pages 15-22, 1987.

[81] M. Zijlema. On the construction of a third-order accurate monotone convection scheme with application to turbulent flows in general domains. International Journal for Numerical Methods in Fluids, 22:619-641, 1996. 